\title{
EXPERImental ACQUiSition AND CharaCterisation of LARGE-Scale Flow Structures in Turbulent Mixed Convection
}

\author{
DISSERTATION \\ zur Erlangung des mathematisch-naturwissenschaftlichen Doktorgrades \\ „Doctor rerum naturalium“ \\ der Georg-August-Universität Göttingen \\ im Promotionsprogramm ProPhys \\ der Georg-August University School of Science (GAUSS)
}

\author{
vorgelegt von \\ DANIEL SCHMELING \\ aus Oldenburg (Oldb)
}

Göttingen 2014 


\section{Betreuungsausschuss}

Prof. Dr. Andreas Dillmann,

Georg-August-Universität Göttingen, III. Physikalisches Institut und Deutsches Zentrum für Luft- und Raumfahrt e.V., Institut für Aerodynamik und Strömungstechnik

Prof. Dr. Andreas Tilgner,

Georg-August-Universität Göttingen, Institut für Geophysik

\section{Mitglieder der Prüfungskommission}

Referent: Prof. Dr. Andreas Dillmann,

Georg-August-Universität Göttingen, III. Physikalisches Institut und Deutsches Zentrum für Luft- und Raumfahrt e.V., Institut für Aerodynamik und Strömungstechnik

Korreferent: Prof. Dr. Andreas Tilgner,

Georg-August-Universität Göttingen, Institut für Geophysik

\section{Weitere Mitglieder der Prüfungskommission}

Prof. Dr. Hans Hofsäss,

Georg-August-Universität Göttingen, II. Physikalisches Institut

Prof. Dr. Ulrich Parlitz,

Georg-August-Universität Göttingen, Institut für Nichtllineare Dynamik und Max-PlanckInstitut für Dynamik und Selbstorganisation

Prof. Dr. Martin Rein, Georg-August-Universität Göttingen, III. Physikalisches Institut und Deutsches Zentrum für Luft- und Raumfahrt e.V., Institut für Aerodynamik und Strömungstechnik

Prof. Dr. Jürgen Vollmer,

Georg-August-Universität Göttingen, Institut für Nichtllineare Dynamik und Max-PlanckInstitut für Dynamik und Selbstorganisation

\section{Weiterer Korreferent}

Prof. Dr. Markus Raffel,

Leibniz Universität Hannover, Institut für Turbomaschinen und Fluid-Dynamik und Deutsches Zentrum für Luft- und Raumfahrt e.V., Institut für Aerodynamik und Strömungstechnik

Tag der mündlichen Prüfung: 02. Juli 2014 


\section{DANKSAGUNG}

Bedanken möchte ich mich bei:

bei Prof. Dr. Andreas Dillmann für das Interesse an der Arbeit, die Diskussionen im Anschluss an die Vorträge sowie der Ermöglichung diese Arbeit am Institut erstellen zu können.

bei Prof. Dr. Andreas Tilgner für die Übernahme des Korreferates und die fachlichen Anregungen nach den Vorträgen.

bei Prof. Dr. Claus Wagner für das Ermöglichen dieser Arbeit in der Abteilung Fluidsysteme sowie den anregenden Diskussionen beim Abfassen der eizelnen Veröffentlichungen.

bei Dr. Johannes Bosbach für die wissenschaftliche Betreuung dieser Arbeit, für die vielen gemeinsammen Diskussionen insbesondere beim Verfassen der einzelnen Veröffentlichungen, für die Ideen bei der experimentellen Umsetzung sowie für das Korrekturlesen.

bei Dr. Andreas Westhoff für die gemeinsamme PIV Messung und das Korrekturlesen.

bei Prof. Dr. Baburaj A. Puthenveettil und Govindaraju S. Gunasegarane vom IIT Madras in Indien für die erfolgreiche Kooperation bei der Messung von Linienplumes in turbulenter Konvektion in Luft und der daraus entstandenen JFM Veröffentlichung.

ins besondere bei Sebastian Wagner, meinem langjährigen Bürokollegen, für viele wissenschaftliche und unwissenschaftliche Diskussionen, das Korrekturlesen, die Ideen, die Anregungen und die sonstige Unterstützung.

Daniel Schiepel und Dr. Matthias Kühn für gemeinsames Lösen experimeteller Probleme, viele Diskussionen sowie zahlreiche Stunden der gemeinsammen Ablenkung.

allen weiteren Kollegen der Abteilung Fluidsysteme des Institutes für Aerodyamik und Strömungstechnik des DLRs in Göttingen. Hervorzuheben seien an dieser Stelle Sven Lange und Katharina Rabe für die Untersützung bei einzelnen Versuchen.

der ersten und zweiten Herren Volleyball Mannschaft des ASCs sowie meinen weiteren Freunden für die regelmäßige Ablenkung von der Arbeit.

meiner Familie, Michael, Renate, Maren und Jonathan Schmeling, für die durchgehende Unterstützung und das Immer-da-sein.

und abschließend natürlich ganz besonders bei meiner Frau Nele, unserer Tochter Hannah und unserem ungeboren Kind. Für das immer an mich glauben, die Tatsache, dass es etwas wichtigeres als Arbeit gibt, die Urlaube, die Ablenkung, die Freude, die vielen gemeinsammen Stunden, den Mut, die Motivation und so vieles mehr.

\section{DANKE}




\section{- for Hannah -}

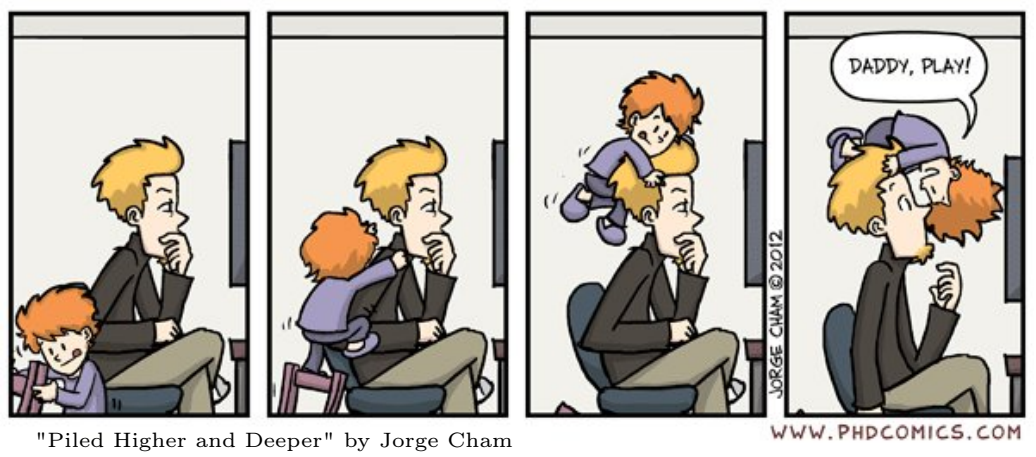

Do never forget what is really important. 
"Attention! There are only two possibilities now: Either it works or it doesn't."

Luke, in "Jim Button and Luke the Engine Driver"

Michael Ende 



\begin{abstract}
In this cumulative thesis, the superposition of shear flow and thermal convection, namely mixed convection, is investigated experimentally. The experiments were performed in a cuboidal sample with aspect ratios $\Gamma_{x z}=$ length/height $=5$ and $\Gamma_{y z}=$ width/height $=1$. The sample was composed of a heatable bottom and coolable ceiling as well as air inlet and outlet channels, which are located on the same side and both span the whole length of the sample. Different measurement techniques were applied and four main results were obtained.

The first main result is the finding of large-scale flow structures, which arrange in different configurations within the sample depending on the characteristic numbers. In specific, the breakdown of a two-dimensional forced convective roll structure at low and vanishing $A r$ into three-dimensional configurations at higher $A r$ was found. Thereby, stable arrangements of three and four thermally induced convection rolls were also found. Their axis of rotation was found to be zigzag shaped and tilted with respect to the inflow velocity. This is ascribed to the superposition of the thermally induced convection rolls with shear forces.
\end{abstract}

The second main result is the observation of a maximum in the enthalpy flux carried by the fluid between in- and outflowing air at $A r \approx 0.6$. This was caused by the existence of an upper boundary of the temperature difference between in- and outflowing air with changing $A r$. As a consequence, there are flow conditions, which maximise the enthalpy flux of the flow through the cell.

The third main result is the finding of different dynamics of the large-scale flow structures, namely stable configurations, erratic changes and periodic oscillations. These results are based on long duration temperature measurements and smoke visualisations. A dependency on the underlying characteristic numbers, namely $R e, R a$ and $A r$, was found: first the strength of the thermal convection $(R a)$ determines if oscillations can occur. Second, mixed convection $(A r)$ determines the dynamics (steady, erratic changes or periodic oscillations) if $R a$ allows oscillations. Third, forced convection $(R e)$ determines the oscillation frequency if $A r$ allows periodic oscillations.

The fourth main result is the experimental realisation of simultaneous measurement of instantaneous temperature and velocity fields in a system with continuous fluid exchange and air as working fluid. This is more a result of the experimental accessibility than of the fluid dynamical processes in mixed convection. However, for the challenging task of measuring both quantities non-intrusively and extensively, a solution is presented, which was developed and successfully applied. The main idea of this technique is the simultaneous usage of thermochromic liquid crystals as tiny thermometers and as tracer particles for particle image velocimetry. Experimental cornerstones are the particle generation, their illumination, image filtering and calibration. They are addressed in this thesis. 
Additional accomplishments of the thesis are the following findings: The large-scale flow structures couple the momentum transport from one wall to the opposing side wall. Even more, a back-coupling is found and a concept describing the mechanisms acting to trigger the periodic oscillations is presented. Furthermore, it showed that a proper adjustment of $R e$, i.e. the inflow velocity, can destroy stable arrangements of large-scale structures or oscillating configurations of them. Finally, probability density functions of the temperature distribution in a horizontal layer, slightly above the bottom thermal boundary layer, allowed for analysis of fingerprints of the sheet-like thermal plumes. For the given location of the measurement plane a change of the plume fraction $P_{2} / P_{1}$ occurred at $R a \approx 2.3 \times 10^{8}$. Here $P_{1}$ denotes the abundance of fluid temperatures imprinted by the bulk flow, while $P_{2}$ inhibits the abundance of temperatures ascribed to warm thermal plumes. 


\section{CONTENTS}

\begin{tabular}{lll}
\hline ABSTRACT & VII
\end{tabular}

\begin{tabular}{lll}
\hline NOMENCLATURE & XI
\end{tabular}

\begin{tabular}{lll}
\hline I & PREAMBLE & 1
\end{tabular}

\begin{tabular}{lll}
\hline 1 & Motivation & 3
\end{tabular}

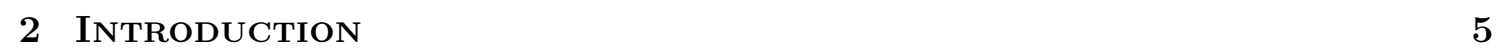

\begin{tabular}{lll}
\hline 3 & COMMON THREAD & 25
\end{tabular}

\begin{tabular}{lll}
\hline II ANALYSIS & 27
\end{tabular}

4 Large-Scale Flow Structures and Heat Transport $|105|$

\begin{tabular}{|ll|l|l|}
5 & Temperature Oscillations in TURbulent MC & 102
\end{tabular}

6 Oscillations of the large-Scale Circulations [98]

7 Temperature AND Velocity Measurements in Convective AIR $\begin{array}{lc}\text { FlOWS [101] } & 87\end{array}$

8 Dynamics of Thermal Plumes in Turbulent MC $|100|$

\begin{tabular}{lr}
\hline III CONCLUSION & 139
\end{tabular}

\begin{tabular}{lll}
\hline & SUMMARY AND CONCLUSION & 141
\end{tabular}

\begin{tabular}{ll}
\hline 10 OUTLOOK & 145
\end{tabular}

\begin{tabular}{lr}
\hline BIBLIOGRAPHY & 147
\end{tabular}

\begin{tabular}{ll}
\hline A CURRICUlum VitaE & 157
\end{tabular} 



\section{NOMENCLATURE}

\section{OFTEN USED ACRONYMS}

2C Two Component

3C Three Component

2D Two-Dimensional

3D Three-Dimensional

DLR Deutsches Zentrum für Luft- und Raumfahrt e.V. / German Aerospace Center

FC Forced Convection

TC Thermal Convection

MC Mixed Convection

RBC Rayleigh-Bénard Convection

LSC Large Scale Circulation

Re Reynolds number

$R a \quad$ Rayleigh number

$\operatorname{Pr} \quad$ Prandtl number

Ar Archimedes number

PIV Particle Image Velocimetry

PIT Particle Image Thermometry

TLC Thermochromic Liquid Crystal

LED Light Emitting Diode

PDF Probability Density Function

POD Proper Orthogonal Decomposition 



\title{
PART I
}

\section{PREAMBLE}

\author{
1 Motivation \\ 2 INTRODUCTION \\ 3 Common Thread
}





\section{Motivation}

Thermal convection is a prevalent mechanism of heat transport. For instance, it drives the development of clouds [121, the transport of thermal energy from the interior to the outer parts of stars [116] and is responsible for the plate tectonics [34] and thus for the occurrence of earthquakes. In addition, it is of fundamental importance for the ventilation of rooms 69].

Furthermore, turbulent convection is one of the basic physical systems in which the fundamentals of turbulence can be investigated. Hence, it has been studied for more than 100 years, yet is still not fully understood.

By mixed convection (MC), one generally means the superposition of thermal convection (TC) and forced convection (FC). Here TC is the result of density gradients induced by temperature differences and FC is applied by an external pressure gradient. MC is characterised by three dimensionless parameters as well as the sample geometry. Commonly, these three dimensionless numbers are the Reynolds $(R e)$, Rayleigh $(R a)$ and Prandtl number $(P r)$. They can roughly be interpreted as the ratio of: inertia to viscosity forces; buoyancy to viscosity forces; as well as momentum to thermal diffusivity, respectively. Further, in literature the Archimedes number $A r=R a \times \operatorname{Re}^{-2} \times \operatorname{Pr}^{-1}$ is found. It expresses the ratio of buoyancy to externally applied inertia forces. The geometry is often characterised in terms of the aspect ratio or aspect ratios $\Gamma_{i}$.

In turbulent convection the flow tends to be unsteady. The resulting coherent structures can possess an oscillatory behaviour or reveal erratic changes. Hence, for a better understanding of the physical mechanisms, the knowledge of the instantaneous velocity and temperature fields is of utmost importance.

In pure TC many, but far from all, processes and their dynamics have meanwhile been observed and deeply studied. Their underlying physics are mainly understood and some of the processes are well described by physically motivated models. MC has more degrees of freedom than TC. Therefore, even though it is more important for technical applications, it is much less investigated. To which extent models and other phenomena are similar in $\mathrm{TC}$ and $\mathrm{MC}$ is still an open issue. The objective of my work is to characterise and analyse the flow, its structure and dynamics. Thereby, I want to identify physical processes of the dynamics of MC. The working fluid for the present investigation is air at ambient pressure and the configuration is a cuboidal sample. The height amounts to $H=500 \mathrm{~mm}$ while length $L$ and width $W$ are given by $L=5 \times H$ and $W=H$.

The approach is to use known measurement techniques for velocity fields (particle image velocimetry, or PIV) and temperatures (temperature probes) as well as to develop 
and apply novel techniques for the simultaneous acquisition of both quantities (PIV and particle image thermography, or PIT). PIV allows the investigation of instantaneous velocity fields. However, it is restricted to rather short measurement times (order of two hours). Temperature probes provide only local information, but allow one to study longterm processes for several days. The novel combination of PIT with PIV is restricted to shorter measurement times than for PIV (order of 10 minutes). However, it facilitates the simultaneous acquisition of both instantaneous temperature and velocity fields.

The objective of characterising the processes of MC, describing its physics and modelling it is far from being completely achieved. In the present thesis the following questions shall be answered in order to approach this aim:

1. How do large-scale circulations (LSCs) arrange in a cuboidal convection sample of aspect ratio five as a function of the characteristic numbers?

2. How does the enthalpy transport between in- and outflowing air depend on the Archimedes number?

3. How can the stability as well as the dynamics of the LSCs be characterised and in which ways do the dynamics scale with the characteristic numbers?

4. How can temperature and velocity fields be simultaneously recorded in a MC airflow?

5. What is the time evolution of, for example, a warm rising plume in this enclosure?

6. How do the Rayleigh and the Archimedes numbers affect the dynamics of sheet-like plumes?

Furthermore, some possible explanations concerning the question of how the stability and the dynamic process of the LSCs can be modelled with reference to the characteristic numbers will be discussed. 


\section{INTRODUCTION}

\subsection{THERMAL CONVECTION}

\subsubsection{A HISTORICAL SURVEY}

In the mid-eighteenth century, George Hadley (1685-1768) and Michail W. LomonoSOV (1711-1765) were (almost simultaneously) the first who recognised the role of nonuniform heating as the producer of fluid motion. While Lomonosov, who was a Russian polymath, worked among other things with mine ventilation, the Englishman Hadley [49] reflected on trade winds. In this context, he proposed that in each hemisphere a thermally driven convection roll could be generated by solar heating.

After these first approaches to thermally driven flows, it was HENRI BÉNARD (1874-1939), who conducted the breakthrough experiment of modern convection research during his $\mathrm{PhD}$ study in 1900 [10]. He studied the formation of convection patterns in a thin fluid layer heated from below, see Figure 2.1.

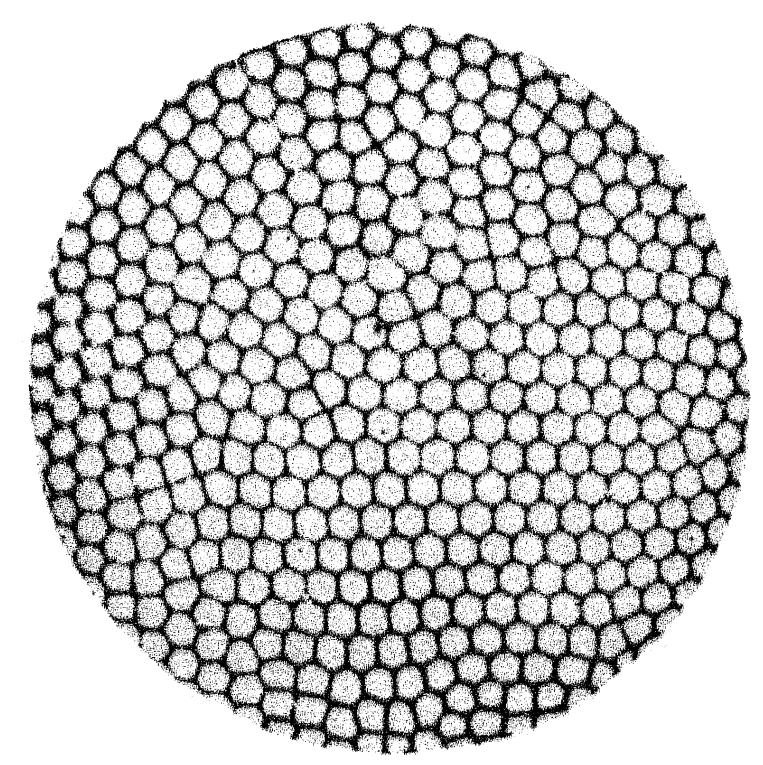

FiguRE 2.1: Pattern formation in Bénard's experiment: spermaceti heated from below with a free upper surface (source: 28] CDover Publications, Inc.).

The theoretical description of Bénard's observations was given by LORD RAYLEIGH (1842-1919) in 1916 [87]. Even though Rayleigh made assumptions concerning the boundary conditions which differ from the original experiment by Bénard, he could describe many 
of the observed effects. Both physicists became namesakes for this type of convection phenomena: 1

In 1879, Anton Oberbeck (1846-1900) [77] and later, in 1903, Joseph V. BoussinesQ (1842-1929) [19], proposed an approximation of the NAVIER-STOKES-equations ${ }^{2}$ which results in a linear buoyancy term and negligence of all further density variations.

The regions of applicability of this approximation were calculated by Gray and Giorgini in 1976 [46] for air and water. Their diagrams depict the limitations of the approximation. For air at mean temperature $T_{0}=15^{\circ} \mathrm{C}$ and ambient pressure, they received $\Delta T \leq 28.6 \mathrm{~K}$ and $H / \Delta T<10.2 \mathrm{~m} / \mathrm{K}$ as restricting temperature difference $\Delta T$ and thickness of the fluid layer $H$, respectively ${ }^{3}$

The introduction of the П-theorem by EdGar Buckingham (1867-1940) in 1914 and 1915 [26, 27 and its interpretation for the fluid dynamics (see e.g. Spurk 117]), is the foundation of the modern analysis of fluid dynamics. The application of the $\Pi$-theorem on the Navier-Stokes equations under the Oberbeck-Boussinesq approximation provides the characteristic numbers, which describe the system together with the geometry entirely.

\subsubsection{IN NATURE AND IN TECHNICAL APPLICATIONS}

In 1735, Hadley 49 hypothesised, that in each hemisphere a convection roll exists which is driven by solar heating and results, among other things, in the trade winds. It turned out that one convection roll per hemisphere is not correct. However, nowadays, it is the accepted scientific view, that convection, driven by temperature gradients is, besides the rotation of the Earth, one of the most important transport mechanisms in our atmosphere. It occurs in the trade winds (Figure 2.2 (a) and [128]), cloud formation, moisture transport and other phenomena (see e.g. the review on atmospheric moist convection by Stevens [121|).

Along with the processes in our atmosphere, convection is also an ubiquitous phenomenon within the earth. Here, the convection of the Earth's mantle governs the generation of plate tectonics (see Figure 2.2 (b) and Davies [34] or Bercovici [11]). It is still an open issue whether full mantle convection or only upper mantle convection occurs, or a combination of both [127]. Furthermore, there are models supporting the contention that convection in the Earth's core plays an important role for the generation of the magnetic field of the Earth (see e.g. the review article on the genesis of the Earth's magnetism from Roberts and King [90]). On larger scales, convection is the driving mechanism for transport of material from the interior to the outer parts, in stars like our Sun (see e.g. Spiegel [116], who proposes various theoretical approaches).

\footnotetext{
1"Historically, the name is inaccurate; Bénard's pioneering observations, although for long believed to relate to this configuration, were actually mostly of another phenomenon - convection due to surface tension variation - that give rise to similar effects" [D.J. Tritton, 1988], 130.

${ }^{2}$ Named after the Frenchman Claude-Louis M.H. Navier (1785-1836) and Irishman Sir Georg G. Stokes (1819-1903), mathematicians and physicists.

${ }^{3}$ That means, that all results and discussions presented in this thesis can be interpreted under the estimation of the Oberbeck-Boussinesq-approximation.
} 


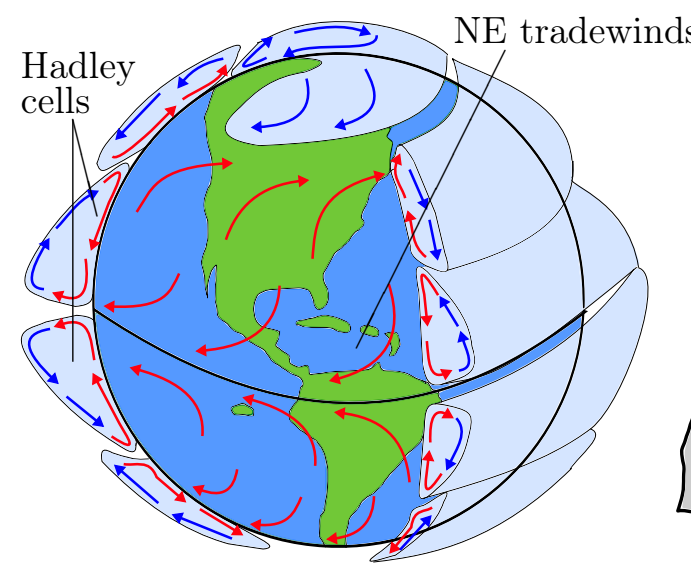

(a)

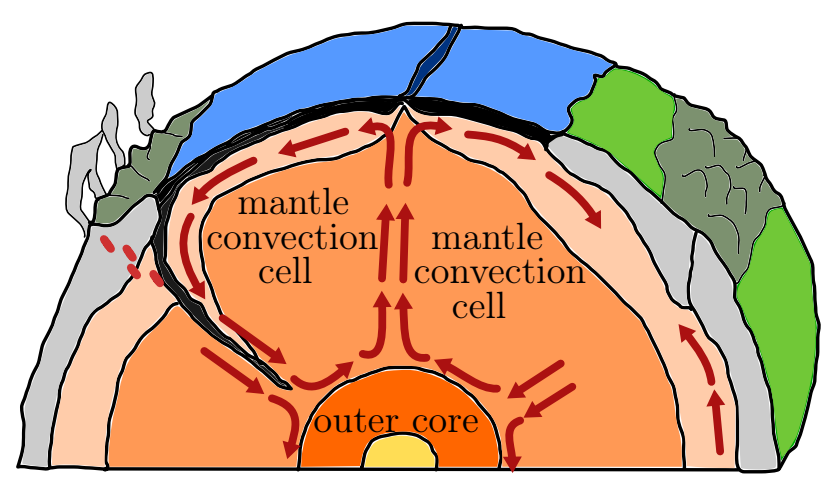

(b)

Figure 2.2: (a) Sketch of convection in the Earth's atmosphere (according to $120 \mid$ ); (b) model of the Earth's mantle convection (according to $[129]$ ).

On Earth, patterns of convective flows are visible, caused by many different phenomena. Figure 2.3 (a) shows structures in a dried out salt lake. Minerals, which are dissolved in a lake, remain when the water evaporates. Then, former three dimensional, mainly hexagonal, convection cells remain as patterns on the surface. Another occurrence of these mainly hexagonal convection cells is visible in basalt columns (see Figure 2.3, b) which develop when basaltic lava congeals quickly. During this cooling on the top side and from the remaining heat on the lower side, the mainly hexagonal convection cells are formed. In the Earth's atmosphere, TC plays a role in cloud formation. It is often superimposed with FC, by wind. In rare cases this interaction of thermal and shear forces, results in cloud formations like the 'Morning Glory', as shown in Figure 2.3 (c). Here, parallel convection rolls form these long cloud tubes in the regions of rising air.

Moreover, convection is one of the most important physical processes in all heat exchanging devices, from the coolant circulation in a nuclear power reactor to the cooling of computer processors with cooling fins or common baking ovens to micro-channel heat sinks to cool e.g. laser diodes or high-performance microprocessors. Exemplarily, the works of Sillekens et al. [110] on MC in a coiled heat exchanger, of Baskaya et al. [7] on cooling electric devices and of Linden [69] on the ventilation of rooms are cited here.

Another important technical application of convection, mainly MC, is the ventilation of interiors. In terms of weight and energy optimisation as well as extreme outer conditions, the air conditioning of an aircraft cabin demands special requirements. Besides the work conducted directly by the manufactures, some aspects are addressed in scientific studies. These range from isothermal ventilation, i.e. pure FC, of generic enclosures 17 to investigations of FC and MC in a full-scale aircraft cabin mock-up 64 and studies on different climatisation scenarios within flight tests [13].

In addition to these technically motivated studies, there are also some biologically motivated ones: Stamatopoulos et al. [119] studied MC in a rectangular tube stenosis, 


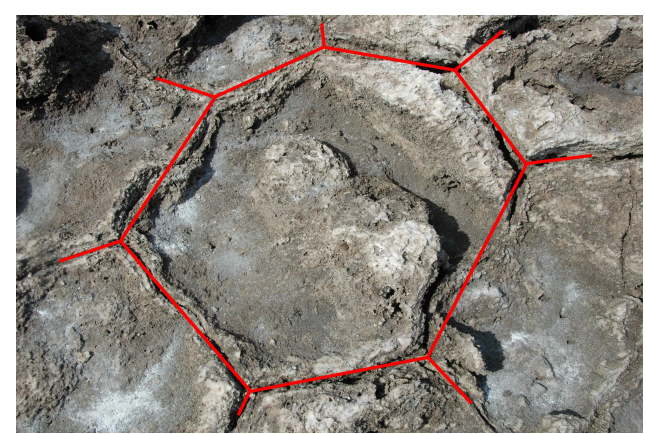

(a)

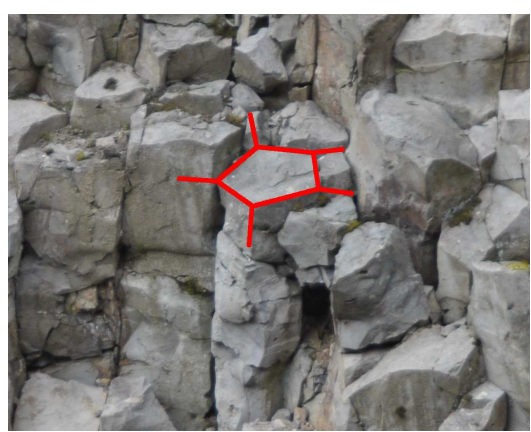

(b)

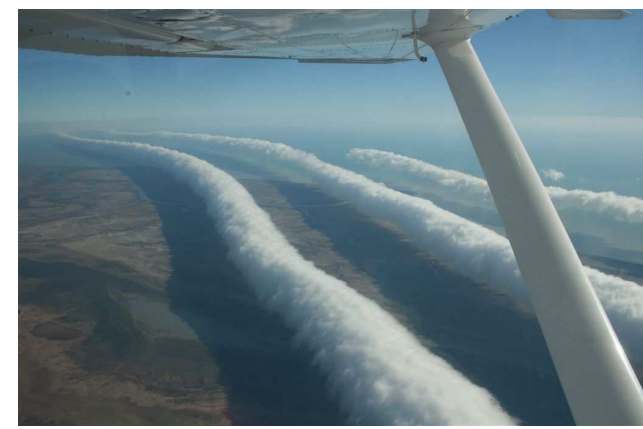

(c)

Figure 2.3: Structure formation: (a) in a dried out salt lake 'Devil's Golf Coarse' in the Death Valley, California, USA; (b) in basalt columns in the basalt mine at Hohen Hagen, Dransfeld, Germany; (c) convective roll structure of the 'Morning Glory', a very special cloud (source: [79], CMick Petroff, license [80]).

i.e. a convergent/divergent channel, which is motivated by the blood flow through an inflammatory arterial lesion.

\subsubsection{IN SCIENCE}

Since the first scientific parameter study by Bénard in 1900 [10], thermal convection, and in specific Rayleigh-Bénard convection (RBC), has become an often investigated system. Consequently, the literature on this topic is vast. This is due to the fact that RBC is one of the fundamental systems in which to study the occurrence of turbulence, the transition from a laminar to a turbulent flow, the formation of patterns and the scaling behaviour of multiple quantities in the turbulent regime.

Recent reviews of turbulent RBC4 are given by Chillà and Schumacher [30], Lohse and Xia [71] and Ahlers et al. [3]. The latter focusses on the heat transfer and the large-scale dynamics. The scaling of the Nusselt number $N u$ and the Reynolds number Re as measure

\footnotetext{
${ }^{4}$ Even though the classical RBC experiment was conducted with a very large ratio of diameter to fluid height, i.e. a very thin fluid layer, and a free surface as upper boundary of the fluid, this configuration with an aspect ratio around unity and a heated bottom and a cooled solid ceiling of the container is referred to as RBC.
} 
for the heat transport and the strength of the large-scale circulation, respectively, are analysed as functions of the Rayleigh number $R a$ and the Prandtl number Pr. Furthermore, aspects like the thicknesses of the thermal and the kinetic boundary layers, non-OberbeckBoussinesq effects and the dynamics of the large-scale circulation are addressed. Chillà and Schumacher [30] focussed on the description and interpretation of the boundary layers and Lohse and Xia [71] discussed the small-scale properties of turbulent RBC.

For further discussion of convection, i.e. of scientific investigations of convective processes, it is reasonable to define dimensionless flow parameters in terms of system relevant quantities [3]. These are the Rayleigh number $5^{5} R a$, which has the interpretation of a ratio of buoyancy to viscosity forces:

$$
R a=\frac{\beta \cdot g \cdot H^{3} \cdot \Delta T}{\kappa \cdot \nu}
$$

the Reynolds number ${ }^{6} R e$, determining the ratio of inertia to viscosity forces:

$$
R e=\frac{U \cdot H}{\nu}
$$

and the Prandtl number ${ }^{7} \operatorname{Pr}$, which is the ratio of the momentum to thermal diffusivity:

$$
\operatorname{Pr}=\frac{\nu}{\kappa} .
$$

Finally, the Nusselt number $8 N u$, which represents a dimensionless heat transport. This describes the ratio of convective heat transport to heat transport of a solid body with the same heat conductivity, i.e. conductive heat transport:

$$
N u=\frac{\Phi_{q} \cdot H}{k \cdot \Delta T},
$$

where $H$ denotes the height of the sample, $\Delta T$ the temperature difference between top and bottom of the sample, $U$ the characteristic velocity, $\Phi_{q}$ the spatially and time-averaged heat flux per unit area, $g$ the acceleration due to gravity. Moreover, the fluid properties are $\beta$ the isobaric thermal expansion coefficient, $\kappa$ the thermal diffusivity, $\nu$ the kinematic viscosity, $c_{p}$ the specific heat capacity (at constant pressure), $\rho$ the density and $k=c_{p} \cdot \rho \cdot \kappa$ the thermal conductivity of the fluid.

Furthermore, the geometry determines the convective flow and heat transfer. In particular, the aspect ratio $\Gamma$ of a cylindrical sample and the aspect ratios $\Gamma_{x z}$ and $\Gamma_{y z}$ of a cuboidal sample:

$$
\Gamma=\frac{D}{H}, \quad \Gamma_{x z}=\frac{L}{H}, \quad \Gamma_{y z}=\frac{W}{H},
$$

where $H$ is the sample's height, $D$ the diameter of a cylindrical samples and $W$ and $L$ are the width and length of cuboidal samples, respectively.

\footnotetext{
${ }^{5}$ Named after the British physicist Lord Rayleigh (1842-1919).

${ }^{6}$ Named after the British mathematician and engineer Osborne ReYnolds (1842-1912).

${ }^{7}$ Named after the German engineer Ludwig PrandTL (1875-1953).

${ }^{8}$ Named after the German engineer E.K. Wilhelm Nusselt (1882-1957).
} 
Besides the geometry, in RBC $R a$ and $P r$ are the only two control parameters, which determine the flow properties like heat transport, i.e. $N u$, and resulting velocity distribution, i.e. Re. In contrast to $\mathrm{RBC}$, in $\mathrm{MC}, R e$ is an additional control parameter. An external pressure gradient applies a characteristic velocity, e.g. the inflow velocity, to the convection system. Hence, the velocity and also Re turns from a passive to an active parameter. Regarding these three active flow parameters $R a, P r$ and $R e$, the Archimedes number ${ }^{9} A r$, turns out to be another important dimensionless flow parameter:

$$
A r=\frac{R a}{R e^{2} \cdot P r} .
$$

It interprets the ratio of buoyancy to inertia forces and therefore indicates whether TC or FC dominates. For the sake of completeness it should be noted that in the case of FC, thermal forces are negligible and only the pressure gradient drives the flow.

A detailed survey on the literature is given in the introduction of the results chapters, i.e. the accumulated articles therein. At this point the cited works just give an overview.

Saeidi and Khodadadi 92 studied FC in a square cavity based on computational fluid dynamics. It has a similar cross section as the here investigated cuboidal sample. Saeidi and Khodadadi studied inlet and outlet configurations similar to ours and observed, under specific parameters, a clockwise rotating LSC (see Figure 5, bottom left, in 92, which was also found in our convection sample). Another investigation on FC was performed by Körner et al. 62]. They studied the flow in a generic room with isothermal obstacles and an inlet and an outlet. Therein, one of their main findings was the existence of coherent oscillations.

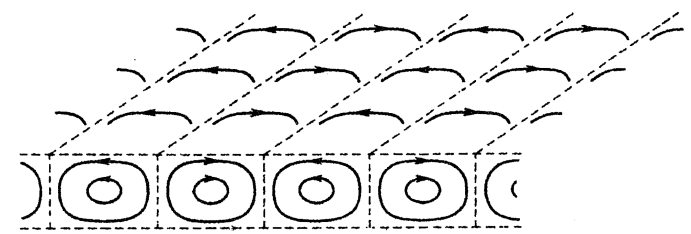

(a)

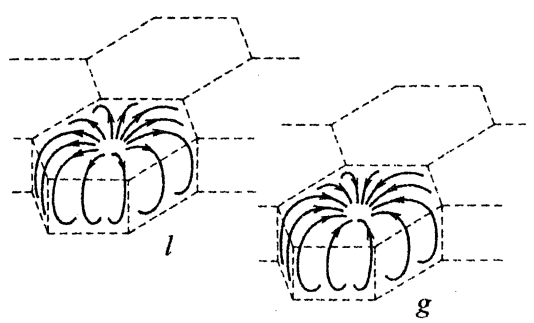

(b)

Figure 2.4: (a) Sketch of the fluid motion in rolls; (b) sketch of the fluid motion in cells (source: 43] C)World Scientific Publishing).

The literature on pure TC is vast. The studies span from pattern formation close above the onset of convection to the scaling laws in highly turbulent convection at many orders of magnitude higher $R a$. The former is discussed in detail by Getling [43], who describes different convection patterns as shown in Figure 2.4. The hexagonal cells, Figure 2.4 (b), correspond to the experiments of Bénard, Figure 2.1, and structures observed in the dried

\footnotetext{
${ }^{9}$ Named after the Greek mathematician, physicist and engineer Archimedes of Syracuse (circa 287 BC-212 BC).
} 
out salt lake and the basalt columns, Figure 2.3 (a,b). The occurrence of the different patterns is discussed deeply in terms of stability analysis.

Recent works on turbulent TC deal with the scaling of $N u$ and $R e$ as functions of $R a$ and $P r$. Here, $N u$ is a measure for the heat transport and $R e$ for the magnitude of the mean wind. Another open question in this context, which is still a matter of debate, is if LSCs in turbulent TC develop from the cellular patterns observed slightly above the onset of convection [2]. To achieve high $R a$, the aspect ratio becomes smaller and is mostly in the order of unity in recent studies. This is contrary to the original experiments of Bénard and the theoretical work of Lord Rayleigh, who assumed an infinitely extensive fluid layer. Several studies reveal different arrangements of the LSCs in the convection sample [138, 149] and varying dynamics $20,24,25,73,150]$.

A cuboidal sample leads to a different system with reduced symmetry, as compared to a cylindrical sample. However, the characteristics of the LSC in such a cuboidal sample at $\operatorname{Pr} \approx 4$ show a dependency on $R a$ as well [151. The aspect ratio is also important for the LSC arrangement in cuboidal samples. Three-dimensional numerical simulations revealed the influence of the depth of a cuboidal sample with equal width and height on the number of LSCs and their arrangement 136. Furthermore, in two-dimensional simulations, the number of LSCs was found to depend strongly on the aspect ratio [133. In case of the present configuration, the formation and dynamics of the LSCs as well as the role of thermal plumes by means of experiments [72, large eddy simulations [81, 106] and direct numerical simulations (DNS) [60], are studied.

Besides the influence of $\operatorname{Pr}, R a$ and the geometry, the boundary conditions play an important role for the development of flow structures and the heat flux. However, a two-dimensional convection sample, periodic in the horizontal direction, was investigated up to $R a=10^{10}$ with constant temperature and constant heat flux boundary conditions at the top and bottom of the sample [59. No influence of the scaling of $N u$ or the time averaged temperature profiles and the dynamics were found. Moreover, there is a heated discussion about the existence of a unifying theory of scales posited by Grossmann and Lohse [48]. They proposed, for eight different $R a-P r$ regimes, power laws for $N u$ and $R e$ depending on $R a$ and $P r$.

$\mathrm{MC}$ is a vastly occurring state, which occurs in nature, in heat exchangers or in the climatisation of rooms. Scientific works examine heated channel flow (also named PoiseuilleBÉNARD flow), see [74, cooling of electric devices, i.e. a forced flow over an arrangement of heat sources, see e.g. [7, 111, or indoor climatisation, see 69. In a geometrical similar generic enclosure, Westhoff [139] analysed the dynamics of coherent structures and their scaling in MC.

Recently, studies, which address a rather generic configuration of room ventilation by means of DNS were presented [5, 109]. They studied the flow within a room with heated obstacles and superimposed FC (setup comparable to the isothermal investigations of Körner et al. 62]). Their considered configuration with four parallelepiped obstacles can also be seen as a generic room or aircraft cabin. Hence, for MC, the transition from purely 
scientific works to those representing technical applications is fluent.

\subsubsection{THE CONVECTION SAMPLE - BETWEEN FUNDAMENTAL PHYSICS AND TECHNICAL APPLICATION}

A sketch of the investigated convection sample is given in Figure 2.5. It is a cuboidal sample and has an air inlet and an outlet on the same side of the cell. Furthermore, the bottom of the sample is heatable and the ceiling is coolable, and thus a vertical temperature gradient can be applied, i.e. TC can be generated. By applying an external pressure gradient between the inlet and outlet, FC can be produced. Running both systems simultaneously results in MC. Thereby, the ratio between the strength of TC and FC, i.e. the ratio of buoyancy to inertia forces, is variable. Besides the square vertical cross section of the cell, i.e. an aspect ratio $\Gamma_{y z}=1$ between $W$ and $H$, the sample has an aspect ratio of $\Gamma_{x z}=5$ between $L$ and $H$. Here, width, height and length of the sample amount to $W=500 \mathrm{~mm}, H=500 \mathrm{~mm}$ and $L=2500 \mathrm{~mm}$, respectively. The inlet channel is located directly below the ceiling whereas the outlet channel is placed directly above the bottom of the cell. Both span the whole length of the sample and their heights aggregate to $H_{\text {in }}=25 \mathrm{~mm}$ and $H_{\text {out }}=15 \mathrm{~mm}$, respectively.

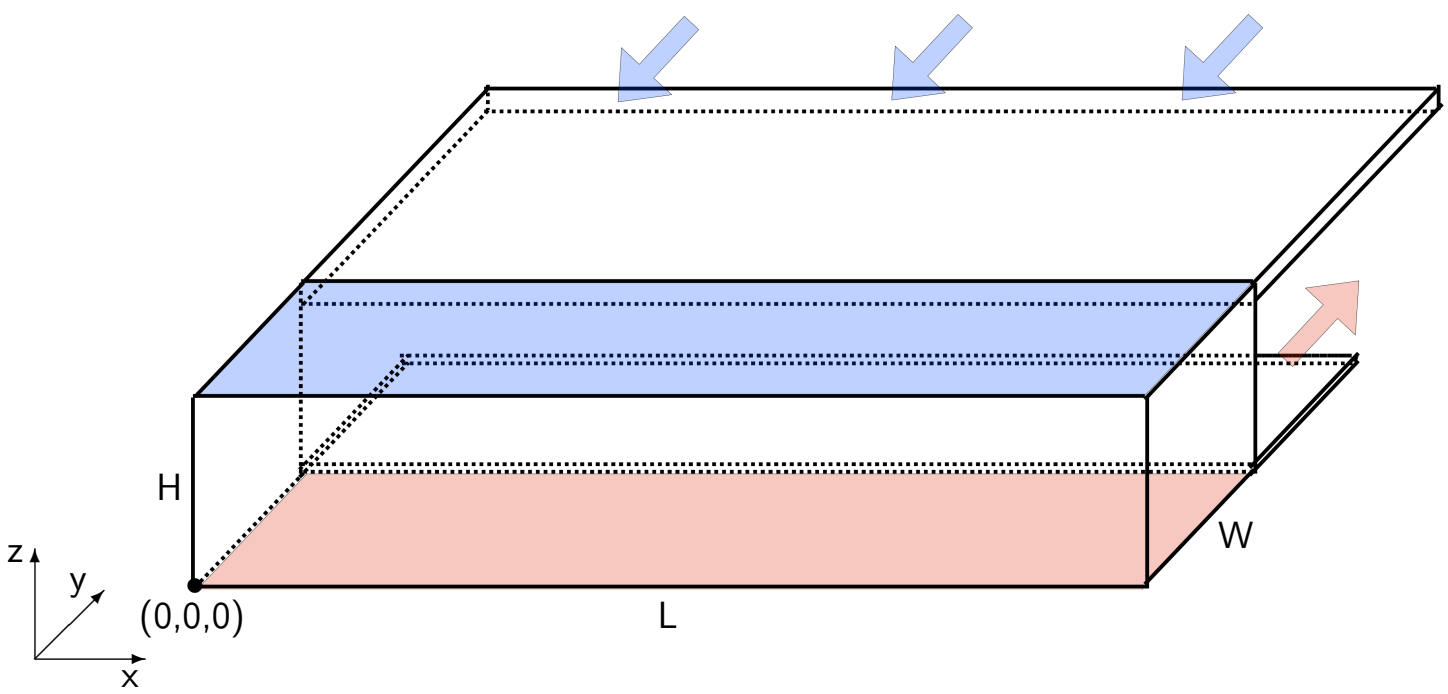

Figure 2.5: Sketch of the convection sample. The bottom plate of the cavity is heated (red) while the ceiling is kept at ambient temperature (blue). The inlet and outlet channels are located on the same side wall and span the whole length of the sample. The blue and red arrows depict the in- and outflowing air, respectively. It has a quadratic cross section, a length of $2500 \mathrm{~mm}$ and the aspect ratio between length and height amounts to $\Gamma_{x z}=5$.

Further, the present configuration can be interpreted as a strongly simplified aircraft cabin. Figure 2.6 shows the evolution from an aircraft cabin, in Figure 2.6 (a), to the classical cylindrical RBC sample in Figure 2.6 (f). In case of the present configuration, the cold air, which enters the cabin at the ceiling, is replaced and substituted by an isothermal cooled plate. Furthermore, all passengers and the interior are substituted by an isothermal 


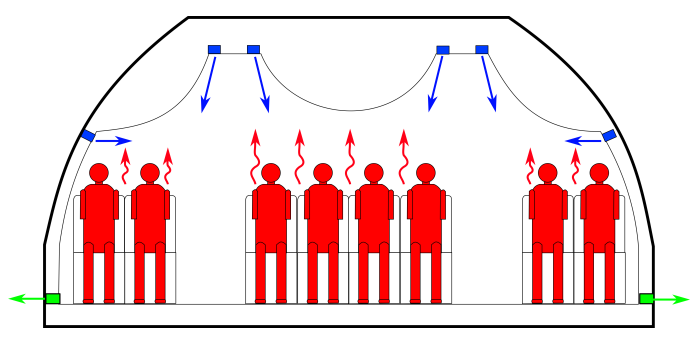

(a)

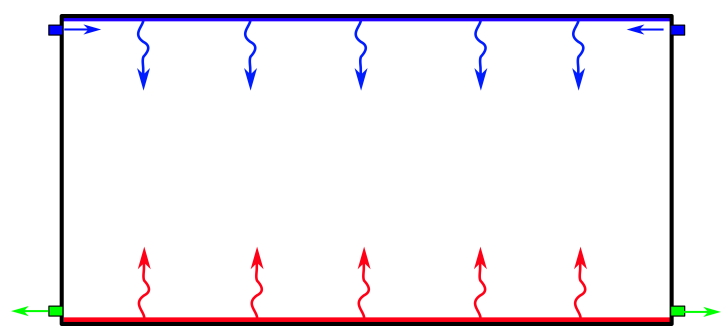

(c)

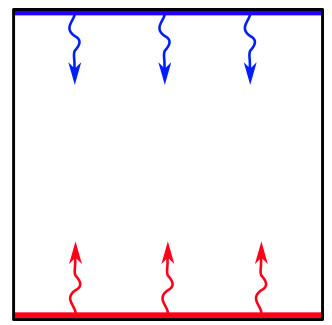

(e)

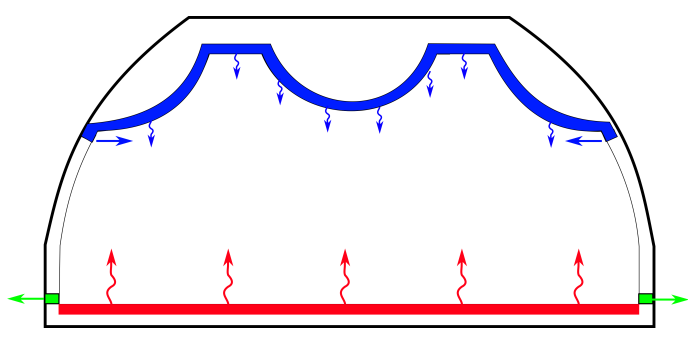

(b)

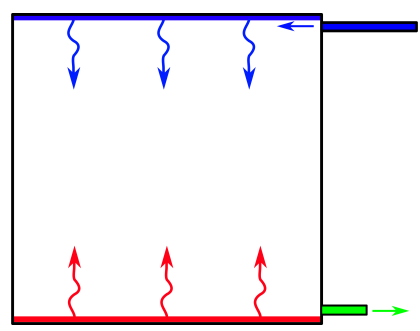

(d)

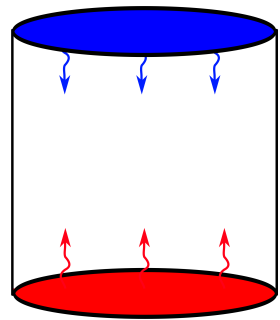

(f)

Figure 2.6: Sketch: The evolution of a convection sample from technical application to fundamental cylindrical RBC: (a) Ventilation of an aircraft cabin; (b) introducing a cooled ceiling instead of all but two air inlets and replacing all passengers by a heated bottom; (c) straightening the geometry; (d) using symmetry: MC in a cuboidal cavity; (e) closing air inlet and outlet and (f) forming a cylindrical container: TC (RBC) in a cylindrical container. Note: even if sketched in two-dimensions, all configurations shall be understood as three-dimensional (Modified, original from [93]).

heated floor. The air inlets and outlets, which are placed at the ceiling and the ground, are surrogated by venting slots (see Figure 2.6, b). Additionally, smoothing of the boundaries leads to the configuration shown in Figure 2.6 (c) and due to the high symmetry, we consider just one-half of the sample (Figure 2.6, d). The configuration represents MC in a squared cross section heated from below and cooled from above with an additional air inlet and outlet. Both slots are located on the same side and spans the whole length of the container. If we further remove the ventilation slots, we obtain TC in a cuboidal sample with a squared cross section (Figure 2.6, e). Finally, reshaping the cuboidal to a cylindrical sample leads to the classical RBC configuration (Figure 2.6, f). Realistic configurations of an aircraft cabin, including e.g. overhead compartments are the subject of just a little number of studies $16,17,64$. In contrast the literature on pure TC in particular, for 
cylindrical samples is vast as already mentioned before. Nevertheless, investigations of the present configuration or similar are topics of other recent studies $66,67,83,139,140$. Thereby, I contribute directly to the work of Westhoff et al. [140] and Puthenveettil et al. [83].

\subsection{MeAsurement TECHNiQUeS}

In the following I will present a survey of the measurement methods which are of relevance. Commencing with a brief historical treatment of the measurement techniques, an introduction of the measurement and visualisation techniques follows. The presentation of the purely qualitative visualisation technique using smoke particles is followed by a section on temperature measurements. These facilitate long-term measurements, however are restricted to invasive point-wise measurements. Subsequently, a section on PIV presents the essentials of the measurement technique for velocity fields. It is followed by a section on PIT. This section is separated into a discussion of the fundamentals of this technique, the well known combined PIT/PIV in liquids and the novel application of the combined technique for air flows. For the sake of completeness, this chapter closes with a section on the volume flow measurement techniques. All mentioned techniques besides the combination of PIT and PIV using TLC tracer particles are already state-of-the-art for air flows. Hence, the given explanations of the techniques raise no claim to completeness. The interested reader may be referred to the given references for deeper information.

\subsubsection{HistoricAL}

Visualisation of fluid flow already fascinated LEONARDO DA VINCI (1452-1519), an excellent artist and intellectual observer of processes in nature. Figure 2.7 shows two of his water studies. The upper one reflects the flow around a planar obstacle in different arrangements. The lower one features swirling structures of water falling into a pool. Even though da Vinci worked centuries before modern flow visualisation began or the first mathematical equations describing the motion of fluids were formulated, he had an eye for the characterising phenomena of the flow, like streaklines or swirls.

Centuries later, Ludwig PRAndTl (1875-1953), see Figure 2.8, founded the branch of research of modern aerodynamics. He realised that the generation of reproducible boundary conditions is of utmost importance for experiments. In 1904 he developed a water channel, the so called Prandtl channel, for the visualisation of flow phenomena. The object of interest is placed within a circulating water channel, which already includes turbulence reducing devices. Aluminium powder is placed on the water-air interface as tracer particles in order to study the flow around the object. In 1908, Prandtl introduced the first wind tunnel in Göttingen, Germany and developed, among others, the airfoil theory.

However, nowadays one does not only want to study flows by observing them, but also to quantify the underlying physical values. 


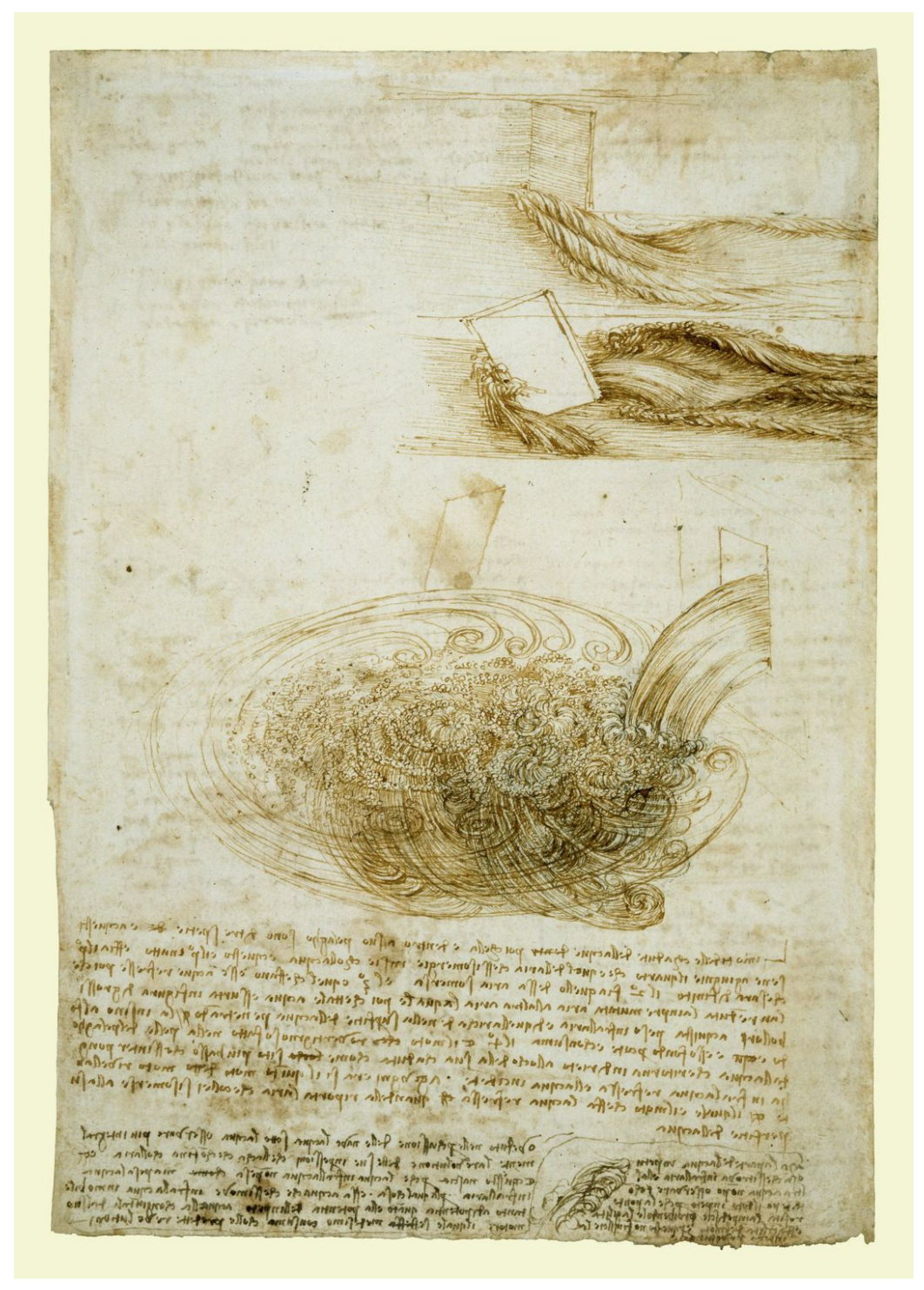

Figure 2.7: This most elaborate of Leonardo's sheets of water studies investigates two basic themes. The first, in the upper two drawings, is the flow of water past a planar obstruction. Below, Leonardo shows the fall of a stream of water from a sluice into a pool, a lucid and highly sophisticated study in which the multiple layered vortices are seen extending far below the surface, each welling current giving rise to concentric circles of bubbles that expand across each other without interference. Leonardo da Vinci (approx. 1510) (source: Caption and image taken from Royal Collection Trust / (C)Her Majesty Queen Elizabeth II 2014 [135|).

\subsubsection{SMOKE VISUALISATIONS}

Smoke visualisation is a very well-known technique for air flows which was already used by Wieselsberger [144] and Prandtl [82] to disclose turbulent structures. In particular, in the case of the early stages of flight and automobile aerodynamics one century ago (see Figure 2.9 this technique was of utmost importance. 


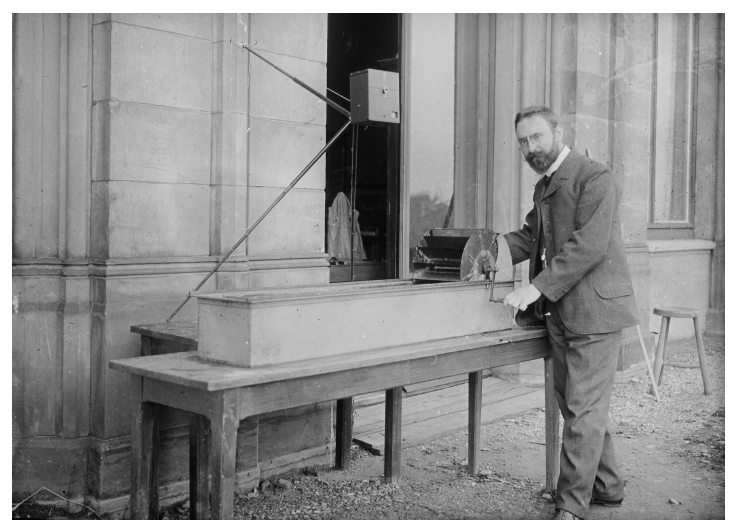

(a)

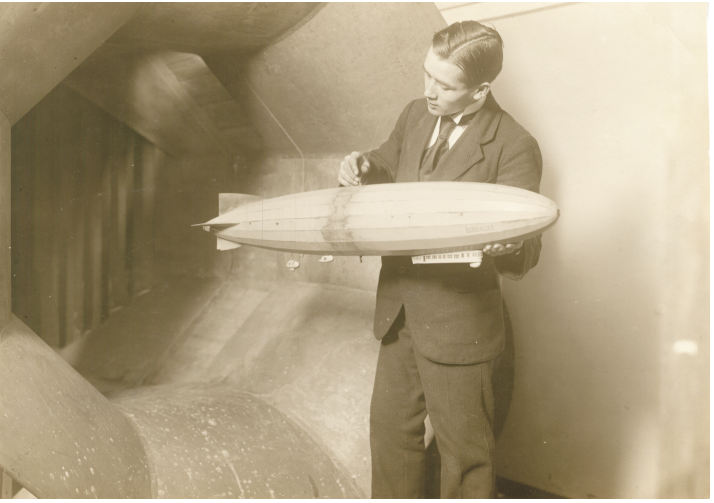

(b)

Figure 2.8: (a) Prandtl in front of the water channel in Hanover (1904); (b) a model of the Zeppelin Airship LZ120 'Bodensee' in the wind tunnel II in Göttingen (1920) (source: $36{ }^{10}$ ).

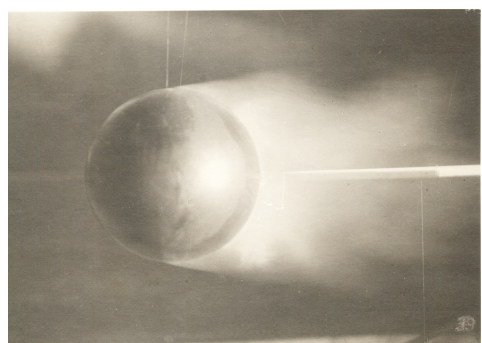

(a)

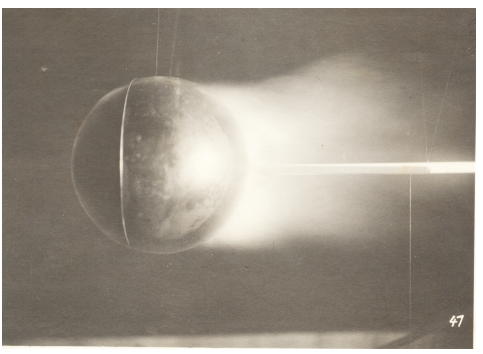

(b)

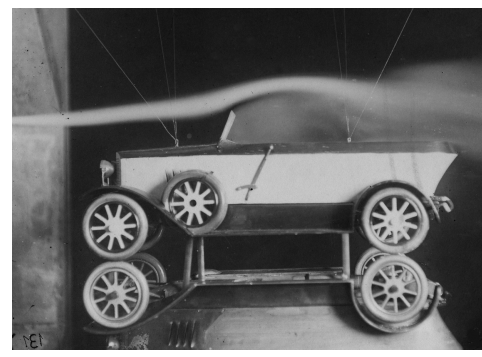

(c)

Figure 2.9: Smoke visualisation of the flow around a sphere (1914): (a) Without a trap wire; (b) with a trap wire; and (c) around a limousine model (1921) (source: $[36]^{11}$.

Mainly two different types of smoke visualisations are known. Either pointwise injection, at a single or at multiple points, of smoke particles combined with a volume illumination or a homogeneous seeding of the inflowing air or the bulk fluid with smoke particles in combination with a laser light sheet illumination. The former is the older technique. It allows for visualisation of streaklines, which collapse for a stationary flow with pathlines and streamlines (see [131], Chapter 11). The latter provides the visualisation of the flow within an illuminated plane. The seeding of the bulk flow, e.g. in a convection sample, facilitates study of the plumes which are formed in the boundary layer and thus appear darker in the laser light sheet. This technique was used for qualitative studies of the line plumes emerging from the bottom thermal boundary layer by Puthenveettil et al. [83]. In case of my studies, smoke visualisation in combination with a laser light sheet was used. The results are published in Puthenveettil et al. [83] and Schmeling et al. [96, 98, 102].

\footnotetext{
${ }^{10}$ The owner of the images could not be located. In case the owner turns up, he or she should get in touch with the author of this thesis.

${ }^{11}$ Please see above note.
} 


\subsubsection{TEMPERATURE MEASUREMENTS}

Temperature measurements, using temperature probes like resistance temperature detectors, e.g. tiny glass bead thermistors, PT-100, or thermocouples, are state-of-the-art for the investigation of thermally driven flows. They provide the advantage of not limiting the measurement time and obviate the optical access to the fluid. However, they only provide the temperatures at specific locations. For the inspection of temperature fields, multiple probes and/or scanning techniques are needed. Furthermore, these scanning techniques provide only time-averaged temperature field information. The interpretation of the results or a combination with other techniques is required to permit statements on the fluid flow. Finally, placing one or more temperature probes in the fluid leads to disturbances. Hence, this technique is an intrusive technique.

In experimental studies, smaller temperature probes, disturbing the flow as little as possible, are preferred. Moreover, such probes provide the fastest reaction times. A disadvantage of these tiny probes is that they usually possess a drift of their calibration function. Thus regular calibration processes have to be conducted or the drawbacks provided by larger probes have to be accepted.

In TC the usage of temperature probes in highly complex convection experiments is widely spread. It is accepted as the measurement technique of choice to investigate TC over long periods of time. A skilful arrangement of the probes within the convection sample can provide insightful statements on the orientation of the LSC and its dynamics.

Here, measurements using temperature probes within the fluid were used for the following publications: Schmeling et al. $95,96,98,102,104,105$.

\subsubsection{PARTiCle image Velocimetry (PIV)}

PIV is nowadays the state-of-the-art technology for the acquisition of velocity vector fields. Fast progress was achieved in the final decades of the twentieth century with the arrival of digital photography. Different types of PIV are currently in use: classical 2C-2D PIV (two velocity components in two dimensions), 3C-2D (also called stereoscopic PIV), multi-colour or scanning PIV and finally tomographic PIV (3C-3D). The latter provides the most information on the velocity distribution of the flow but the complexity of the application increases significantly.

Instead of citing the first works and successive milestones of this technique, I want to refer the reader to a book by Raffel et al. 84]. It provides a comprehensive collection of previous works (almost 500 references) and, furthermore, discusses most of the tasks when applying PIV. Nevertheless, a short introduction of the working principle of $2 \mathrm{C}-2 \mathrm{D}$ PIV is presented in the following.

The fundamental task of PIV is to make cross correlations of two subsequent particle images in many small image sections, so-called interrogation windows. Tracer particles are introduced to the fluid. A challenging issue is the generation of tracer particles, which follow the flow without having a significant momentum on its own, i.e. they should be neutrally 
buoyant. A double pulse laser with light sheet optics illuminates the measurement plane twice with a defined time lag. The cross correlation provides each interrogation window with the most probable displacement vector. It is converted into a velocity vector using the time lag between the two recordings and the magnification factor of the imaging optics. As distinguished from particle tracking velocimetry, where individual particles are tracked, a higher particle density is needed for this technique. A sketch of the measurement setup is shown in Figure 2.10.

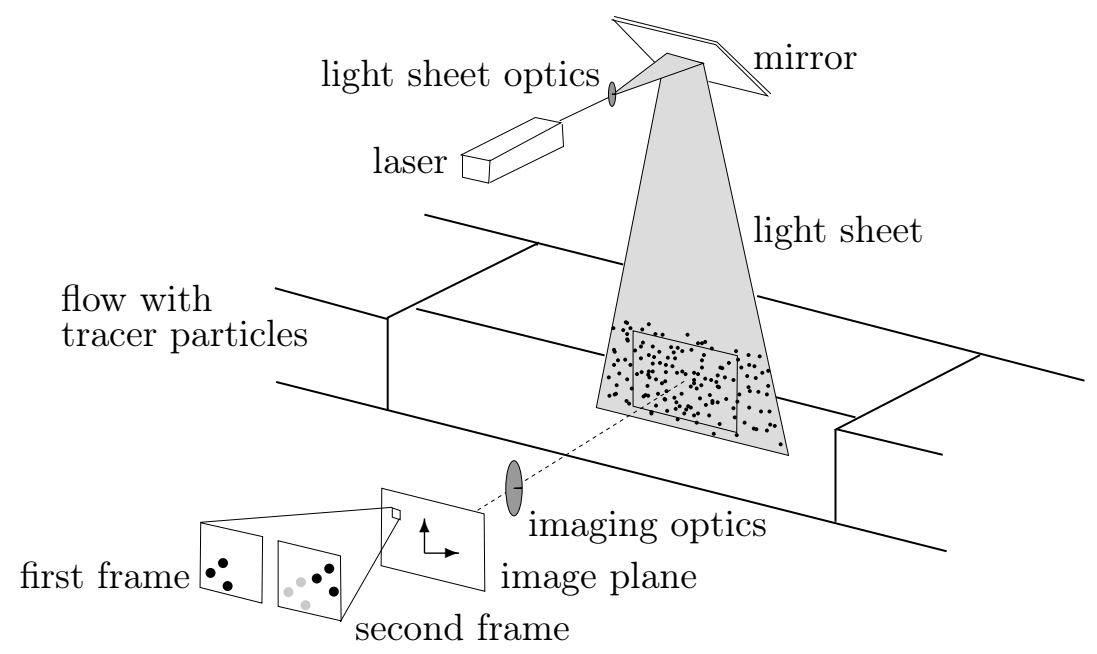

Figure 2.10: Sketch of the measurement setup of a 2C-2D PIV, according to 84 .

The results of my studies using PIV are published in Schmeling et al. 95, 100, 101, 104, 105 and Westhoff et al. [140].

\subsubsection{Particle image thermography (PIT)}

PIT is a method using temperature-sensitive particles, which have, for example, a temperature dependent colour or a temperature dependent emission (and/or absorption) intensity. This material is either placed on a surface as a coating, or it is added as small particles to the fluid flow. Hence it acts as a surface or a fluid thermometer, respectively.

Different realisations are possible, but for my studies, thermochromic liquid crystals (TLCs) in the form of small particles are used as fluid thermometers. For the sake of completeness other promising techniques like laser induced fluorescence [115], the usage of thermographic phosphorous particles $[1,38$ and acoustic tomographic imaging of the temperature [6], shall be mentioned here.

\section{THERMOCHROMIC LIQUID CRYSTALS (TLCS)}

The Austrian chemist Friedrich Reinitzer (1857-1927) was the first to discover (in 1888) the phenomenon that some cholesterol-based substances seem to have two melting points. Melting the solid crystalline structure at $145.5^{\circ} \mathrm{C}$ results in a cloudy liquid; at $178.5^{\circ} \mathrm{C}$ the blurring vanishes and a clear liquid remains [75, 88. Отто LeHmanN (1855-1922), 
a German physicist and an expert in crystal optics, concluded from the observations of Reinitzer and his own studies on Reinitzers' compounds, that the cloudy liquid must possess a kind of molecular structure in contrast to normal liquids. Due to the existence of double refraction, only known in solid crystals so far, he named the new phase 'liquid crystalline' phase and substances which show this phase 'liquid crystals', or LCs [88].

In 1961 the Nobel Prize in physics was awarded to the French theoretical physicist Pierre-Gilles de Gennes (1932-2007). He was honoured for "discovering that methods developed for studying order phenomena in simple systems can be generalised to more complex forms of matter, in particular to liquid crystals and polymer" 76.

The usage of LCs which change their behaviour once they are exposed to an electric field is nowadays wide spread in display technology and was awarded the German Future Prize in 2003.

In general LCs can be categorised into the class of soft matter and possess characterising properties of both liquids and crystals. In contrast to normal substances, LCs do not change phase from solid to liquid at melting point. However, they become liquid crystalline at melting point before changing to normal liquids at the clearing point. In this liquid crystalline phase the materials are liquid, while having anisotropic properties, in contrast to normal liquids. These anisotropic properties are based on a long-range orientation order of the molecules.

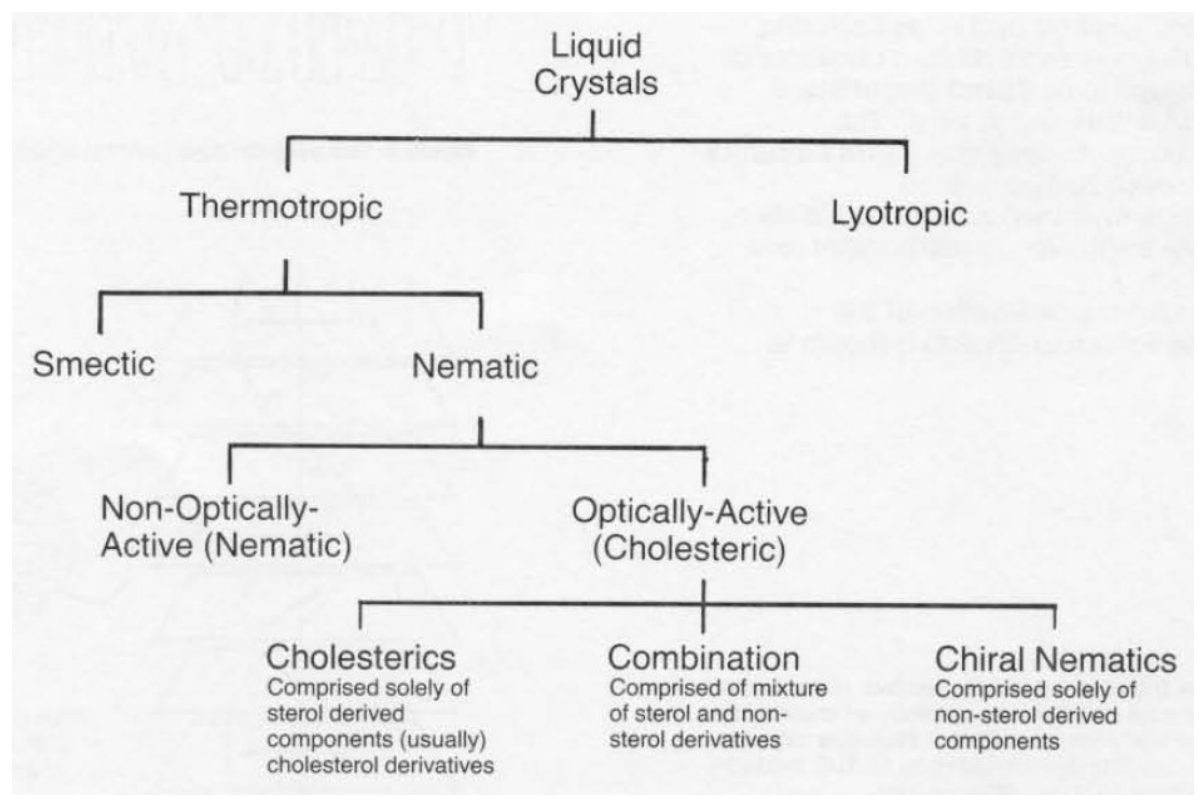

FiguRE 2.11: Schematic representation of the classification of liquid crystals (source: 50 CLCR Hallcrest).

Those LCs, which react to temperature are called TLCs. A schematic representation of LC classification is shown in Figure 2.11. Chemically, the TLCs are organic compounds with rod-like or disc-like molecules. A chemical formula for a rod-like chiral nematic LC molecule is shown in Figure 2.12. Cholesteryl ester LC substances consist of disc-like molecules. 
However, they will not be described here, because only chiral nematic LC substances are used for my studies.

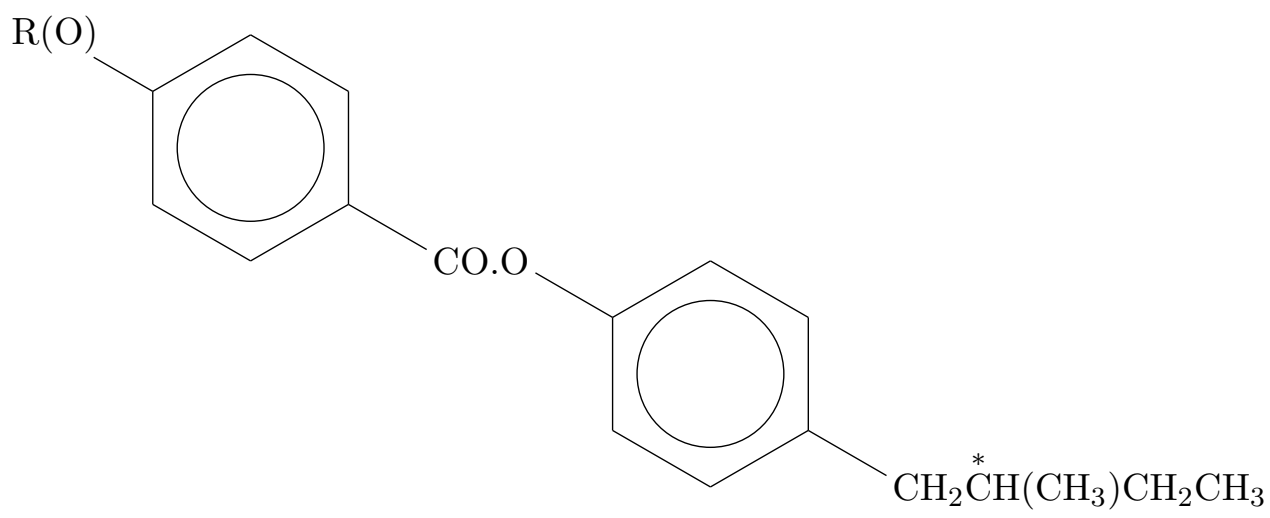

Figure 2.12: Chemical formula of a chiral nematic liquid crystal: (2-methylbutyl) phenyl 4alkyl(oxy)benzoates, where $\mathrm{R}$ is most commonly a straight chain alkyl group. Formula taken from [50].

Once a chiral nematic TLC substance is in the optically active liquid crystalline phase, the molecules are organised in layers. Within these they are arranged parallel to one another. The predominant orientation direction of the molecules in a layer is described by the director $\vec{n}$. This leads to an anisotropic optical medium and the director corresponds to the optical axis. If, furthermore, the director is rotated by a small angle between two neighbouring layers, see Figure 2.13 (a), incident unpolarised white light will be split into two linearly polarised portions. They travel at different velocities while their axes of polarisation rotate quickly. Additionally, according to Bragg scattering by solid crystals, the reflected wavelengths depend on the angle of the view. The important length for the Bragg reflection is the pitch length $P$. It is the length at which the director undergoes a full turn.

Due to thermal expansion in solid crystals the pitch changes only in a minor degree. In contrast to solid crystals, the pitch of TLCs changes due to two opposing mechanisms: Increasing temperature leads to an increasing molecular distance, which increases the pitch. Additionally, the displacement angle of the director between two neighbouring layers $\Theta$ strongly increases with rising temperature. Consequently, it leads to a decreasing pitch. In almost all used TLC substances, the latter process outweighs the other processes [50]. Thus, an increasing temperature leads to a decreasing pitch and to a decrease of the reflected wavelength. More specifically, under a given viewing angle, different wavelengths will be preferentially reflected as a function of temperature. This is illustrated in Figure 2.13 (b). The picture shows the wavelength of the reflected light, as a function of the temperature.

Moreover, the wavelength, i.e. the colour, of the reflected light does also depend on e.g. the size of the particles. Hence, for each experimental setup a colour-temperature calibration has to be conducted.

Almost 30 years ago, Hiller et al. [53,54 presented the first experiments using TLCs as temperature sensitive tracer particles to visualise the convection in a liquid. 


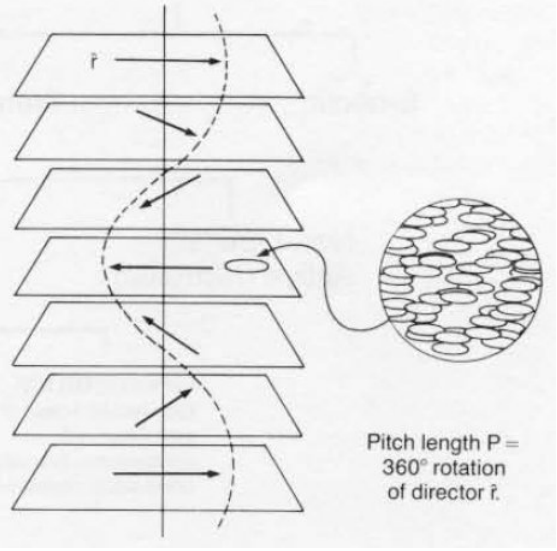

(a)

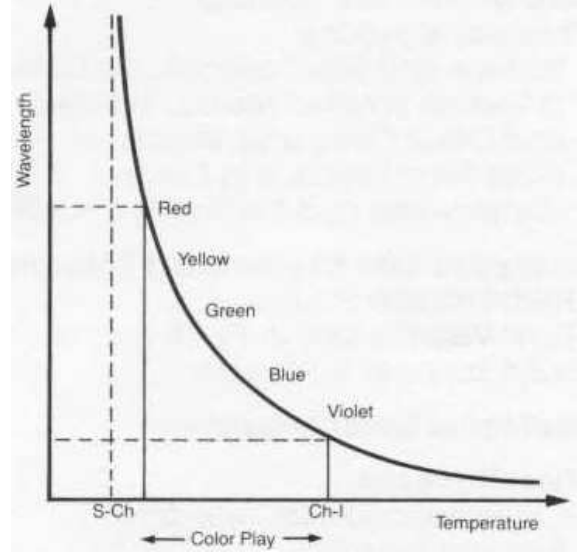

(b)

FigURE 2.13: (a) Representation of a chiral nematic liquid crystal structure: The director (arrow) traces out a helical path within the medium; (b) schematic dependence of the reflected wavelength on the temperature (source: [50 CLCR Hallcrest).

A decade later, Kowalewski 63 recorded a multi-exposed photograph of TLCs, showing a very bright colourplay of the crystals. He investigated a convective glycerol flow in a differentially heated cavity, with a warm left and a cold right side. The temperature field of a LSC structure is shown in Figure 2.14. The blue and the red tints correspond to warm and cold temperatures, respectively. The flow structure as well as the relative temperature distribution are clearly detectable.

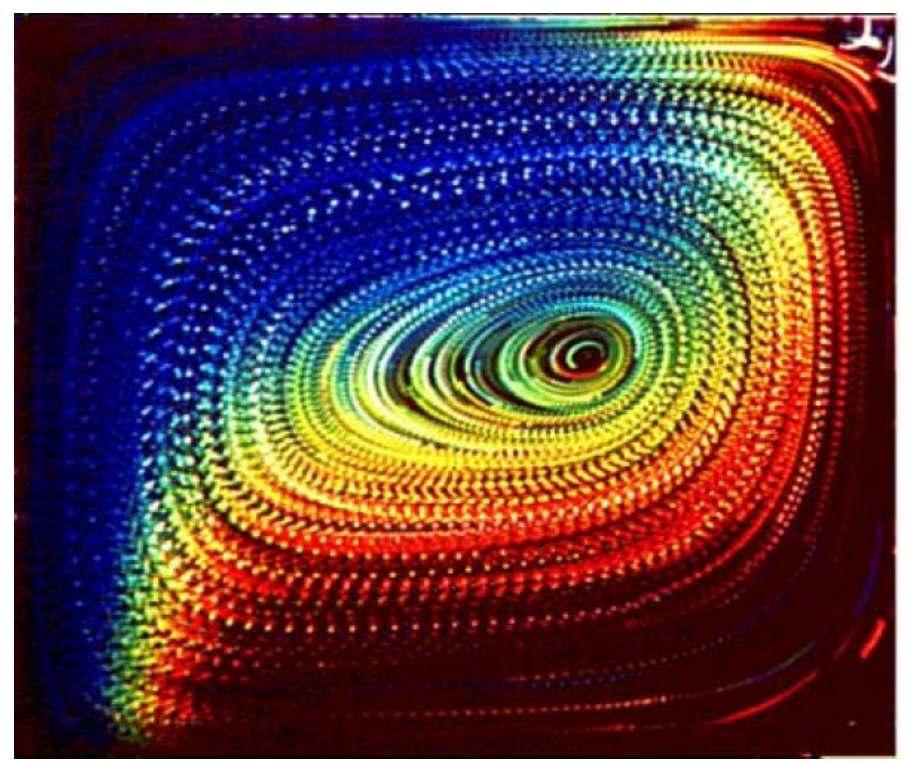

Figure 2.14: Multi-exposed photograph of TLCs in glycerol in a differentially heated cavity (warm left side and cold right side) (source: $\left.63,126{ }^{12}\right]$.

\footnotetext{
${ }^{12}$ The owner of the copyright could not be determined, not even with the help of the author. In case that the owner turns up, he or she should get in touch with the author of this thesis.
} 


\subsubsection{COMBINED PIT/PIV}

Many combinations of measurement techniques promise the simultaneous measurement of both temperature and velocity fields. All PIT techniques mentioned at the beginning of the previous section constitute, in combination with PIV, different ways to realise simultaneous thermometry and velocimetry. They all were developed at the beginning of the twenty-first century. In contrast to these techniques, we adopted PIT using TLCs as tracer particles in combination with PIV from liquid flows to air flows. A detailed description is only given for the combination of PIV and PIT using TLCs, as this is the technique for MC air flows that was developed here.

For a survey of the current state of research of combined digital PIT and PIV, the reader is referred to the recent review given by Dabiri [33]. Nowadays, the technique is established for the investigation of thermal liquid flows. The high amount of effort involved, in particular for the calibration of the TLCs, makes this technique still challenging to use. An example of simultaneous temperature and velocity field measurements is presented in Figure 2.15. Fujisawa et al. [42] investigated a water sample heated from below. They used TLCs as tracer particles and recorded the images in both a vertical plane and a horizontal plane. Accordingly, they made statements on the regions where the formation of convection patterns are located.

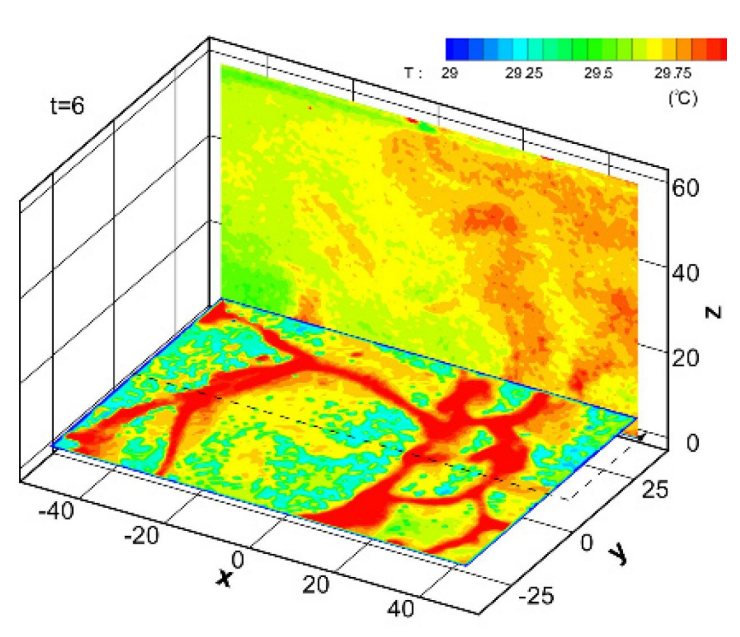

(a)

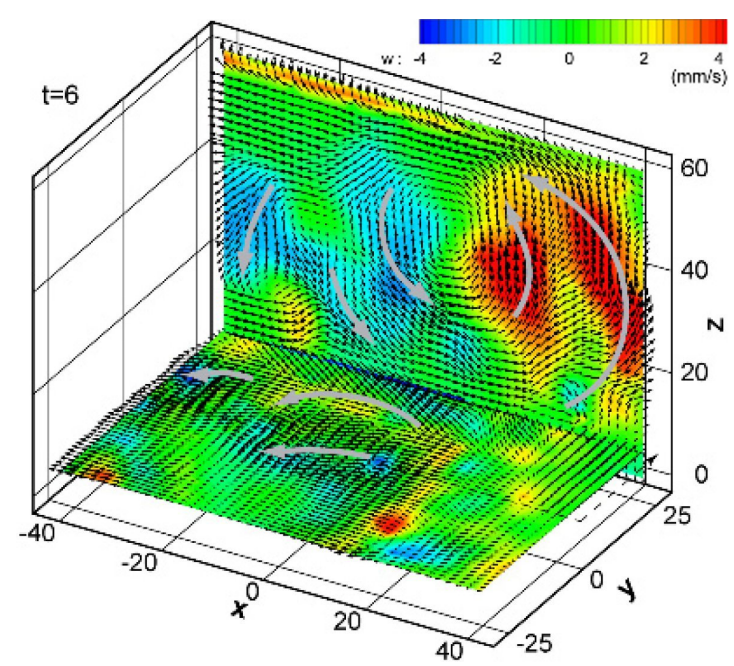

(b)

Figure 2.15: Convection in a water sample heated from below: (a) Temperature; and (b) velocity fields. Note that the vertical plane through $y=0 \mathrm{~mm}$ is the measurement plane, but it is shown in the rear of the image as not to block the results shown on the horizontal plane (source: 42 (C)Springer-Verlag).

In gaseous flows this technique was not available until a feasibility study was performed and patented by Czapp and Czapp et al. 31,32. They instigated RBC in a cubic sample. The further adaptation of this technique to MC, i.e. including a continuous air exchange was conducted in the frame of this work. The usage of the TLCs for combined PIT and 
PIV in airflows is mainly restricted by the size of the TLC particles. On the one hand, the particles must be as small as possible in order to provide the best possible following behaviour, and on the other hand they must be large enough in order to provide a good colour play. Estimations result in suitable particle diameters for air flows of the order of $10 \mu \mathrm{m}$. This is about one order of magnitude smaller than those used in liquids. Accordingly, such bright colours (see Figure 2.14) will not be achieved and a lot of work is needed for particle generation and illumination and the image filtering processes.

Details for this purpose and the application of combined PIT/PIV are published in: Schmeling et al. 94, 95, 100, 101, 103.

\subsubsection{VOLUME FLOW MEASUREMENTS}

To determine the mean inflow velocity, i.e. the Reynolds number, volume flow measurements were conducted. Two different types of volume flow measurement techniques were performed for the investigation of MC. The first method is based on the pressure drop at an orifice plate which is placed in a long straight pipe (see Figure 2.16, a). The volume flow is calculated using the fluid properties and the differential pressure over the orifice combined with correction terms due to flow separation. The empirical correction terms are determined by the International Organisation for Standardization (ISO) which defines this technique in the standard ISO 5167.

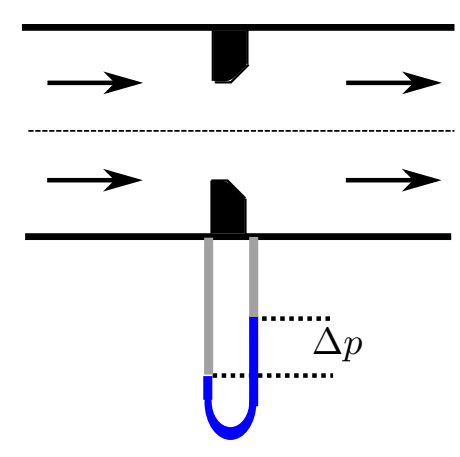

(a)

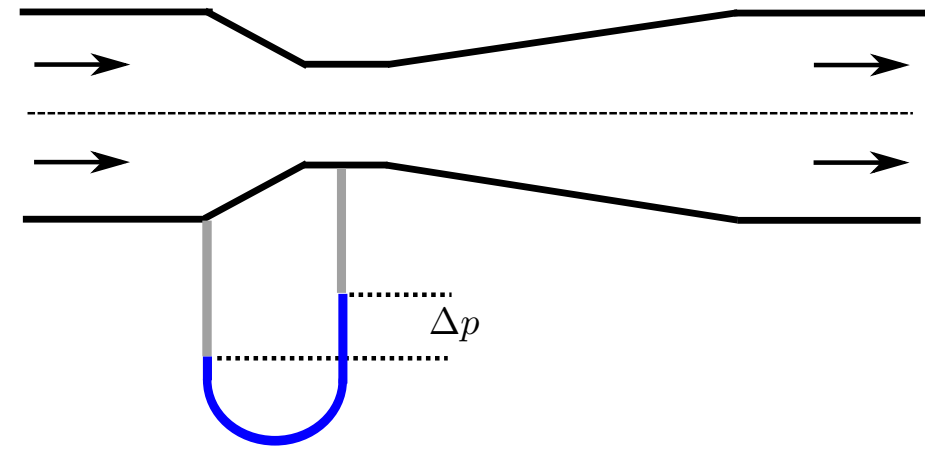

(b)

Figure 2.16: Volume flow measurements: (a) Sketch of an orifice plate; (b) sketch of a Venturi nozzle.

The second technique is based on the principle of measuring differential pressure using a Venturi nozzle ${ }^{13}$, instead of the orifice, see Figure 2.16 (b). The mathematical tools of the Bernoulli equation ${ }^{14}$ enable the calculation of the flow velocity neglecting friction effects. The main advantage of this technique is the smaller loss of pressure. A flow meter which utilises this principle is, for example, the VSM-02 flow meter from Optiserv Messtechnik GbR.

\footnotetext{
${ }^{13}$ Named after the Italian physicist Giovanni Battista Venturi (1746-1822).

${ }^{14}$ Named after the Swiss mathematician and physicist Daniel Bernoulli (1700-1782).
} 
Results based on volume measurements using a flow meter based on one of these techniques are published in: Puthenveettil et al. [83], Schmeling et al. 96, 98, 100, 102, 104, 105] and Westhoff et al. [140]. 


\section{Common Thread}

The accumulated publications in the following chapters are parts of my contribution to characterising the physical mechanisms in MC. The six questions, listed in Chapter 1, are discussed, where certain issues are addressed in particular chapters.

Chapter 4 "Large-Scale Flow Structures and Heat Transport of Turbulent Forced and Mixed Convection in a Closed Rectangular Cavity", published in the International Journal of Heat and Fluid Flow 105] addresses the issue: "How do LSCs arrange in a cuboidal convection sample of aspect ratio five as a function of the characteristic numbers?" as well as "How does the enthalpy transport between in- and outflowing air depend on the Archimedes number?". The PIV technique and temperature probes in the air inlet and outlet are utilised to determine the global heat transfer. As a result, transitions from two-dimensional flow structures, which occur at low Ar, to three-dimensional structures are observed. These latter are composed by the mutual interplay of the two-dimensional FC roll and the TC rolls, the rotation axes of which are oriented parallel to the incoming air. Furthermore, an upper boundary for the temperature difference between in- and outflowing air was found, which results in a maximum of the enthalpy flux between the inlet and outlet of the sample.

Chapters 5 and 6" "Temperature Oscillations in Turbulent Mixed Convective Air Flows", published in Turbulence and Interactions in Notes on Numerical Fluid Mechanics and Multidisciplinary Design 102 and "Oscillations of the Large-Scale Circulations in Turbulent Mixed Convection in a Closed Rectangular Cavity", published in Experiments in Fluids 98 discuss the long-term transient processes which are expected to occur in MC. For both studies, measurement times in the order of one day and more were realised. These were conducted using temperature probes within the fluid and smoke visualisations for the interpretation of the temperature signals in terms of flow structures. The question "How do LSCs arrange in a cuboidal convection sample of aspect ratio five as a function of the characteristic numbers?" is discussed here as well. In addition, the question "How can the stability as well as the dynamics of the LSCs be characterised and in which ways do the dynamics scale with the characteristic numbers?" is debated. The existence of temperature fluctuations within the sample on large time scales was found. The latter were correlated to spontaneous reconfigurations and periodic changes of the number of the roll structures. The different regimes: stable, spontaneous reconfigurations and periodic oscillations, were each ascribed to an effective Archimedes number. It was observed, for the cases with periodic oscillations that the maximal frequency scales linearly with the Reynolds number (based on 
the inflow velocity). Additionally it was found that the large-scale roll structures coupled the momentum transport between the side walls. In this process, it is of importance for many technical applications that such oscillations can be suppressed by modification of the inflow velocity. Finally, a concept is presented, which describes the dynamic processes of the oscillating structures.

Chapter 76"Simultaneous Measurement of Temperature and Velocity Fields in Convective Air Flows", published in Measurement Science and Technology [101] presents a possibility to measure the temperature and the velocity fields in air flows simultaneously. This is of utmost interest, since the formation and the dynamics of the flow structures as well as the heat transport, etc. in convective flows are determined essentially by both the temperature and velocity distributions. Consequently, I worked on the adaptation and development of combined PIT and PIV using TLCs for MC air flows. This article addresses the question "How can temperature and velocity fields be simultaneously recorded in a MC airflow?". The challenges of the combination of both techniques in air flows are discussed and different possible solutions are presented. In specific, different particle generators, the development of a white-light-sheet source based on light-emitting diodes (LEDs), a filter algorithm to reduce the background noise, a dynamic calibration technique as well as a quality analysis of the novel measurement technique are discussed. Furthermore, the question: "What is the time evolution of, for example, a warm rising plume in this enclosure?", is addressed applying initial measurements using PIT/PIV. The temperature and velocity fields of a thermal plume are shown at high spatial and temporal resolution.

Finally, Chapter 8 "Dynamics of Thermal Plumes in Turbulent MC" addresses the question: "How do the Rayleigh and the Archimedes numbers affect the dynamics of sheetlike plumes?". Here, the extended opportunities for the experimental analysis of convection flows provided by the application of PIT/PIV are applied. Hence, new insights in the physical processes, which determine MC, are achieved. The thermal fingerprints of sheet-like plumes and the stems of mushroom-like plumes are visualised in instantaneous temperature and velocity fields. For a deeper analysis of the statistics of the plumes, probability density functions (PDFs) of the temperature are shown. A sum of two Gaussian curves is used to fit the distribution functions. Studying the ratio of the probabilities $P_{2} / P_{1}$ revealed a sudden change at a critical $R a_{c} \approx 2.3 \times 10^{8}$. Here $P_{1}$ denotes the abundance of fluid temperatures imprinted by the bulk flow, while $P_{2}$ inhibits the abundance of temperatures ascribed to warm thermal plumes. Accordingly, $P_{2} / P_{1}$ is a measure for the plume fraction in the measurement plane. The change occurs in the $A r$ regime $2.7 \lesssim A r \lesssim 3.3$, in which the interaction of buoyancy induced LSCs with the wall jet of the incoming air results in an instability. The importance of this intermediate $A r$ regime is confirmed by an analysis of the angle $\alpha$ of the mean flow, which reveals a dependency on $A r$ only. 


\section{PART II}

\section{ANALYSIS}

4 Large-Scale Flow Structures and Heat Transport [105

5 Temperature Oscillations in Turbulent MC [102]

6 Oscillations of the Large-Scale Circulations 98

7 Temperature and Velocity Measurements in Convective Air

FLows 101

8 Dynamics of Thermal Plumes in Turbulent MC 100 



\title{
4 LARGe-Scale Flow Structures AND HEAT TRANSPORT 105
}

Citation and credit: Reprinted with permission from International Journal of Heat and Fluid Flow, volume 32, pages 889-900, 2011, DOI:10.1016/j.ijheatfluidflow. 2011.06.006, Copyright 2011, Elsevier Inc.

Reference: 105

Title: "Large-Scale Flow Structures and Heat Transport of Turbulent Forced and Mixed Convection in a Closed Rectangular Cavity"

Authors: Daniel Schmeling, Andreas Westhoff, Matthias Kühn, Johannes Bosbach and Claus Wagner

Contributions: I designed and built the corresponding experiment together with Matthias Kühn. I performed all measurements together with Andreas Westhoff, I analysed them and made all figures, besides Figure 4.7. Figure 4.8 and Figure 4.9, I wrote the first draft, whose structure has been preserved.

\begin{abstract}
Results of an experimental study of flow structure formation and heat transport in turbulent forced and mixed convection are presented. The experiments were conducted in a rectangular cavity with a square cross section, which has an aspect ratio between length and height of $\Gamma_{\mathrm{xz}}=5$. Air at atmospheric pressure was used as working fluid. The air inflow was supplied through a slot below the ceiling, while exhausting was provided by another slot, which is located directly above the floor. Both vents extend over the whole length of the cell. In order to induce thermal convection the bottom of the cell is heated while the ceiling is maintained at a constant temperature. This configuration allows to generate and study mixed convection under well defined conditions. Results of forced convection at $R e=1.07 \times 10^{4}$ as well as mixed convection at $1.01 \times 10^{4} \leq R e \leq 3.4 \times 10^{4}$ and $R a=2.4 \times 10^{8}(3.3 \geq A r \geq 0.3)$, which
\end{abstract}


were obtained by means of Particle Image Velocimetry and local temperature measurements, are presented. For purely forced convection a 2D mean wind, which can be approximated by a solid body rotation, is found. With increasing Archimedes number this structure becomes unstable, leading to a transition of the solid body rotation into additional smaller convection rolls. Proper orthogonal decomposition of the instantaneous velocity fields has been performed for further analysis of these coherent large-scale structures. Their fingerprint is found in the spatial temperature distribution of the out flowing air at the end of the outlet channel, which reveals a temporally stable profile with two maxima over the length of the outlet. Moreover a maximum in the global enthalpy transport by the fluid is found at $A r \approx 0.6$.

\section{LIST OF SYMBOLS}

\begin{tabular}{ll|ll}
$A r$ & Archimedes number & $c_{\mathrm{p}}$ & specific heat capacity \\
$G r$ & Grashof number & $g$ & acceleration of gravity \\
$N u$ & Nusselt number & $h$ & enthalpy flux \\
$\operatorname{Pr}$ & Prandtl number & $H$ & height of the cell (char. height) \\
$R a$ & Rayleigh number & $k$ & heat transmission coefficient \\
$R e$ & Reynolds number & $L$ & length of the cell \\
$\beta$ & thermal expansion coefficient & $\Delta Q$ & difference of thermal energy \\
$\Gamma$ & aspect ratio & $\Delta T$ & temperature difference \\
$\kappa$ & thermal diffusivity & $\Delta t$ & time interval \\
$\lambda$ & wavelength & $U, v_{\text {in }}$ & characteristic velocity \\
$\tilde{\lambda}$ & normalised eigenvalue & & (mean inflow velocity) \\
$\nu$ & kinematic viscosity & $(\bar{u}, \bar{v}, \bar{w})$ & mean velocity \\
$\phi$ & eigenfunction & $\left(u_{\mathrm{i}}, v_{\mathrm{i}}, w_{\mathrm{i}}\right)$ & instantaneous velocity \\
$\Phi$ & magnitude of the eigenfunction & $\dot{V}$ & volume flow rate \\
$\rho$ & density & $W$ & width of the cell
\end{tabular}

\subsection{INTRODUCTION}

Convective flows are usually categorised into three regimes: If the transport of heat is driven by a thermally induced density gradient the flows are termed Thermal Convection (TC), whereas the transport of heat driven by a pressure gradient is called Forced Convection (FC). Mixed Convection (MC) denotes the intermediate regime where both, TC and FC, are involved.

The system considered here is characterised by five dimensionless parameters, i.e. the Reynolds number $R e=\frac{U \cdot H}{\nu}$, the Grashof number $G r=\frac{g \cdot \beta \cdot \Delta T \cdot H^{3}}{\nu^{2}}$, the Prandtl number $\operatorname{Pr}=\frac{\nu}{\kappa}$ as well as the aspect ratios of the rectangular container $\Gamma_{\mathrm{xz}}=\frac{L}{H}$ and $\Gamma_{\mathrm{yz}}=\frac{W}{H}$. Here $\beta$ denotes the isobaric thermal expansion coefficient, $g$ the acceleration due to gravity, 
$\kappa$ the thermal diffusivity, $\nu$ the kinematic viscosity, $\Delta T$ the applied temperature difference, $U$ the characteristic velocity, $H$ the height, $L$ the length and $W$ the width of the cell. In addition to these numbers, one often refers to the Rayleigh number $R a=G r \cdot \operatorname{Pr}$ if the flow is confined between two horizontal plates, heated from below and cooled from above. Another parameter, which is very useful for the characterisation of $\mathrm{MC}$ is the Archimedes number. It is defined by $A r=\frac{R a}{R e^{2} \cdot P r}$ and is a measure for the ratio between buoyancy and inertia forces. In cases where buoyancy forces are much larger than inertia forces $(A r \gg 1)$ TC dominates and if, in contrast, inertia forces are much larger than buoyancy forces $(A r \ll 1)$ FC dominates. The regime of MC is characterised by the fact that buoyancy and inertia forces are of the same order of magnitude.

The literature on pure TC is vast. A recent overview on the heat transfer and the dynamics of large-scale structures in turbulent Rayleigh-Bénard convection was given by Ahlers et al. [3] with a focus on the dependency of $N u$ on $R a$ and $P r$ as well as the scaling of the boundary layers. The process of structure formation in a shallow rectangular cavity was visualised by Lir and Lin [70], who studied the development of rolls oriented parallel to the short side wall of the cavity as a function of $R a$. At slightly supercritical buoyancy the whole cell was found to be filled with these parallel roll structures. Sun et al. 125 observed the occurrence of an oscillatory motion in the bulk region caused by the spatial organisation of thermal plumes in turbulent Rayleigh-Bénard convection in a water filled cylindrical enclosure with an aspect ratio of one by means of Particle Image Velocimetry (PIV). At $R a=7.0 \times 10^{9}$ a large-scale circulation was found to be a result of the motion of plumes, which drive the flow mainly in the first half of their lifetime and are driven by the mean flow mostly in the last half of their life cycle. The spatial coherence of the bulk velocity oscillation could be correlated to the rise and fall of thermal plumes, e.g. a hot plume rising on the left side of the cell forces the bulk to move to the right side. Another study, which is strongly related to our work is given by Xia et al. [151, who measured the $R a$ dependency of the rotary mean wind in a water filled cubic container heated from below and cooled from above in the range $9 \times 10^{8}<R a<9 \times 10^{11}$. Thereby, the term mean wind is used for flow structures with dimensions comparable to the spatial boundaries. A main result of their study is the finding that the mean wind has a low-velocity rotary core. Moreover, a formation of an inner rotating core inside of an outer rotation shell was detected for $R a>10^{10}$. The rotational frequencies of the mean wind and the inner core were found to scale with different exponents for $R a$.

In addition to the characteristic numbers $R e, R a$ and $\operatorname{Pr} \mathrm{TC}$ is strongly influenced by the boundary conditions. Ishihara et al. [58] determined, numerically and experimentally, the spatial temperature distribution of Rayleigh-Bénard convection in a rectangular enclosure filled with silicon oil by means of thermo-sensitive liquid crystals. Depending on the temperature difference between the heating and cooling plates as well as the depth of the cell three kinds of flow patterns are observed: A steady, quasi- two-dimensional flow, a steady, three-dimensional flow and an unsteady flow.

Pure FC in a two-dimensional square cavity was studied by Saeidi and Khodadadi 92 
in a similar configuration as discussed in this paper by means of Computational Fluid Dynamics (CFD). They observed a shift of the detachment point of the wall jet from the top wall towards the inlet for increasing $R e$.

Next to TC or FC the most commonly occurring phenomenon is MC, which is the governing mechanism in e.g. cloud formation, global ocean currents [121] and transport of thermal energy in stars [116]. Furthermore it is responsible for plate tectonics in the bowels of the earth [11] or the allocation of warm air in a heated room [69].

$\mathrm{MC}$ is a frequently studied phenomenon, but many research groups concentrate on complex geometries with technical or geological interest, like aircraft cabins [14,64, heat exchanger [110], different arrangements of heat sources [7] or MC over a heated wavy lower surface 68 .

Besides all these detailed studies of pure TC and MC in specific geometries, some of the basic physical mechanisms regarding the structure formation in $\mathrm{MC}$ are far from being completely understood. Therefore, the objective of the presented work is twofold. First we want to generate an experimental data base containing velocity fields with high spatial resolution for the validation of a scaling theory for turbulent MC [141]. Second we want to contribute to an improved understanding of the fundamental physical mechanisms in MC. In order to study the superposition of pure FC and TC with optical measurement techniques under well-defined boundary conditions, a rectangular convection cell was developed. Within the cell investigations of flow structure formation and heat transport in turbulent MC are performed with air as working fluid. First results are presented in [104 and 140, 142].

In the present paper we present and discuss results of twocomponent, two-dimensional (2C-2D) and three-component, two-dimensional (3C-2D) PIV. In addition, measurements of the temperatures of the outflowing air obtained for different flow conditions $(\mathrm{Pr}=0.72$, $\Gamma_{\mathrm{xz}}=5, \Gamma_{\mathrm{yz}}=1,0<A r \lesssim 3.3$ and $\left.1.01 \times 10^{4} \lesssim R e \lesssim 3.4 \times 10^{4}\right)$ are discussed. In order to identify the topology of the coherent structures - in particular the large-scale circulations - we analysed the instantaneous velocity vector fields using a Proper Orthogonal Decomposition (POD).

The paper is outlined as follows: Section 4.2 describes the experimental setup. In Section 4.3 the experimental results of PIV and temperature measurements as well as the POD analysis are presented. The compulsive conclusion is given in the last section.

\subsection{EXPERIMENTAL SETUP}

\subsubsection{ConVection CELL}

MC was studied in a rectangular convection cell of length $L=2500 \mathrm{~mm}$ with the aspect ratios $\Gamma_{\mathrm{xz}}=\frac{L}{H}=5$ and $\Gamma_{\mathrm{yz}}=\frac{W}{H}=1$, where $H$ denotes the height and $W$ the width of the container. A sketch of the cell is shown in Figure 4.1. In order to accomplish nearly isothermal boundary conditions the heating device at the bottom consists of five separated sub-plates, each of them being actively heated via a temperature controlled water circuit. 
The ceiling is passively cooled close to ambient temperature via an air-air heat exchanger. The side panels are a double-wall system of polycarbonate plates which guarantee good optical access as well as minimal heat loss. The heat transmission coefficient of the side walls was estimated to amount $k \approx 0.96 \mathrm{~W} / \mathrm{Km}^{2}$, so that the side wall boundary conditions are nearly adiabatic. By operating the heating plates a vertical temperature gradient can be applied to the fluid and thus TC can be induced. Furthermore, the cell is equipped with an air inlet at the top and an air outlet at the bottom. The height of the inlet and the outlet is $25 \mathrm{~mm}$ and $15 \mathrm{~mm}$, respectively. Both are located on the same side and extend over the full length of the container. This configuration allows to generate a flow driven by the pressure gradient between in- and outlet. In order to achieve a homogeneous inflow the external ventilating system consists of a settling chamber in front of the inlet as well as an array of honeycombs with a length of $100 \mathrm{~mm}$ and a cell size of $3 \mathrm{~mm}$ in front of the cell inlet. With this setup we are able to generate MC with $R a<6 \times 10^{8}$ and $R e<1 \times 10^{5}$. Here the cell height is chosen as the characteristic length and the mean inflow velocity as the characteristic velocity of our system.
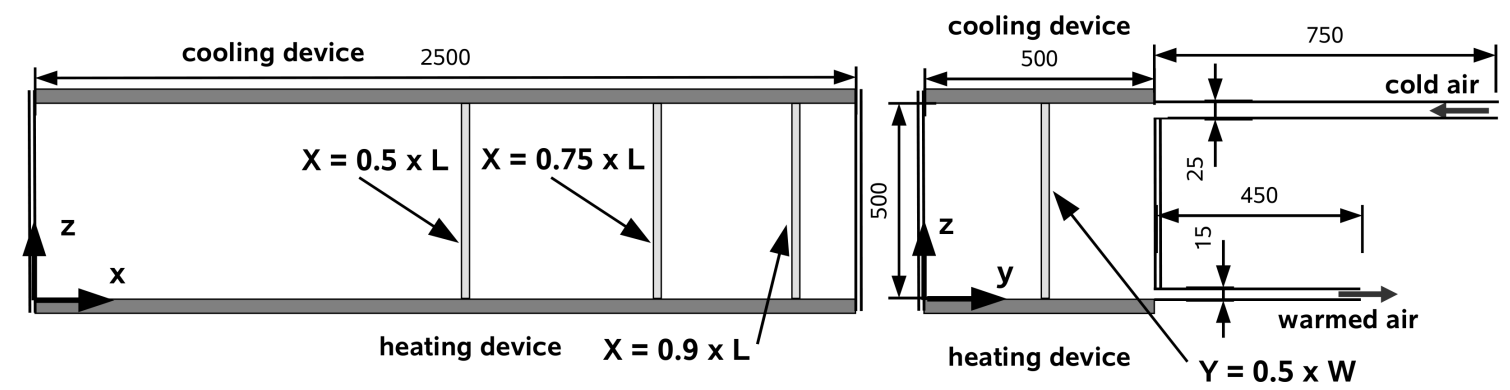

FigurE 4.1: Sketch of the convection cell with the length given in $\mathrm{mm}$. Left: The positions of $2 \mathrm{C}-$ 2D PIV measurement planes at $X=0.5 \times L, X=0.75 \times L$ and $X=0.9 \times L$. Right: The position of the 3C-2D PIV longitudinal measurement plane at $Y=0.5 \times W$.

\subsubsection{MeAsurement Systems}

Temperature measurements were performed with 62 Pt100 (1/ 3 DIN B) temperature sensors at a sample rate of $f \approx 1 / 6 \mathrm{~Hz}$. The cooling and heating plates were equipped with 25 sensors each, and they revealed spatial temperature deviations of $\Delta T_{\mathrm{c} \text {,std }}=1.2 \%$, $\Delta T_{\mathrm{c}, \max }=1.4 \%$ as well as $\Delta T_{\mathrm{h}, \mathrm{std}}=1.1 \%, \Delta T_{\mathrm{h}, \max }=2.2 \%$. Here the suffix $\mathrm{h}$ denotes the heating and $\mathrm{c}$ the cooling device, while the indices std and max denote standard and maximal deviations. All temperature deviations are normalised with the temperature difference between the heating and the cooling plates averaged in space and time, i.e. $\Delta T=21.16 \pm 0.07 \mathrm{~K}$. The $2 \mathrm{D}$ spatial temperature distributions of the cooling and the heating plates are depicted in Figure 4.2 (a) and (b), respectively. They show the time averaged temperatures of the 25 sensors, which are marked as black dots. It is found that the respective maximal lateral temperature differences on the cooling and the heating plate 
were smaller than $\pm 0.5 \mathrm{~K}$.

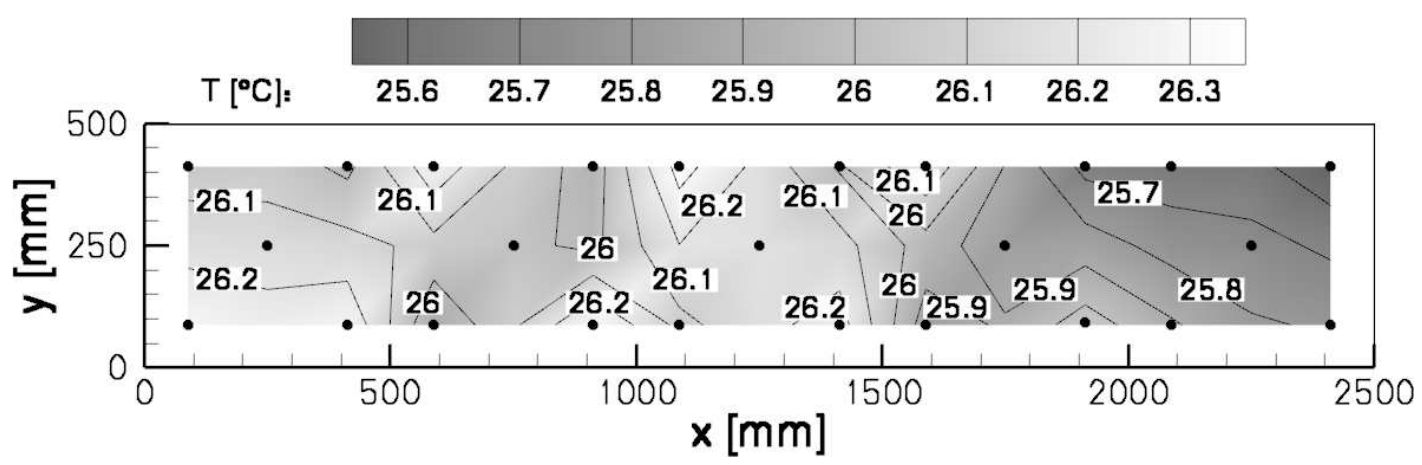

(a)

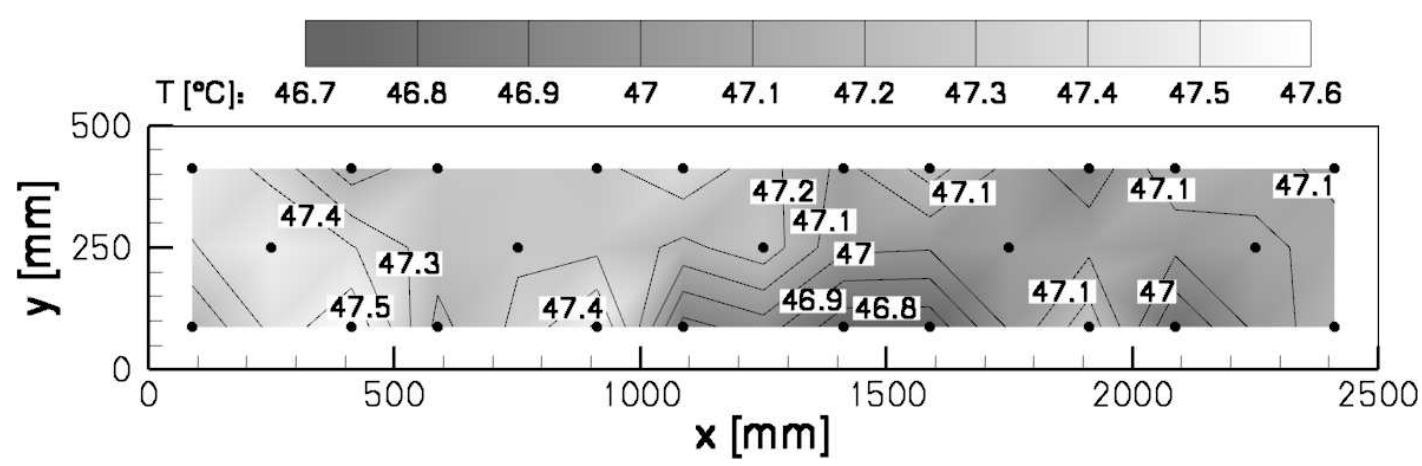

(b)

FiguRE 4.2: 2D time-averaged temperature distributions with isothermal lines of the cooling plate (a) and the heating plate (b) at $A r=3.3$. Temperatures were measured at the positions which are marked as black dots.

The temperature of the incoming air was measured by three sensors which show a deviation averaged in space and time of $\Delta T_{\mathrm{in}, \mathrm{std}}=0.7 \%$ and $\Delta T_{\mathrm{in}, \max }=0.9 \%$. To measure the temperature deviations of the outcoming air, nine sensors were installed equidistantly over the full length at the end of the outlet channel.

In order to determine instantaneous velocity fields 2C-2D and 3C- 2D PIV was applied. For further information on this measurement technique see [84. Small oil droplets with an average diameter of $d \approx 1 \mu \mathrm{m}$ were used as tracer particles. They were illuminated by a dual cavity Nd:YAG laser with a maximum pulse energy of $340 \mathrm{~mJ}$. The light pulses were shaped into a light sheet with a thickness between $2 \mathrm{~mm}$ and $5 \mathrm{~mm}$ by a telescope and a cylindrical lens. In the case of 2C-2D PIV image acquisition was realised with one and in the case of 3C-2D PIV with two CCD-cameras (pco.4000, PCO AG) with a resolution of $4008 \times 2672$ pixel. For $2 \mathrm{C}-2 \mathrm{D}$ and $3 \mathrm{C}-2 \mathrm{D}$ PIV the cameras were equipped with one $f=100 \mathrm{~mm}$ lens (Makro- Planar T*2/100, Zeiss AG) without Scheimpflug adapter and two $f=85 \mathrm{~mm}$ lenses (Planar $\mathrm{T}^{*} 1.4 / 85$, Zeiss AG) with Scheimpflug adapters, respectively. For each series of PIV 500-4800 instantaneous velocity fields were measured with a frequency of $2 / 3 \mathrm{~Hz}$ in four different measurement planes, see Table 4.1 and Figure 4.1 . 
TABLE 4.1: PIV measurement series.

\begin{tabular}{lcl}
\hline & $\begin{array}{l}\text { Number of instantaneous } \\
\text { velocity fields }\end{array}$ & $\begin{array}{l}\text { Position of the measurement } \\
\text { planes }\end{array}$ \\
\hline FC, 2C-2D PIV & 500 & $X=0.5,0.75$ and $0.9 \times L$ \\
FC, 3C-2D PIV & 800 & $Y=0.5 \times W$ \\
MC, 2C-2D PIV & 4800 & $X=0.5$ and $0.9 \times L$ \\
MC, 3C-2D PIV & 4800 & $Y=0.5 \times W$ \\
\hline
\end{tabular}

The evaluation of the 2C-2D images was performed with PIVview2C (PIVTEC GmbH) with a single-pass algorithm using double correlation [52]. A three-point Gaussian-peak fit was used for detection of the correlation maximum with sub-pixel accuracy. The interrogation window size amounted to $32 \times 32$ pixels and the overlap was set to $50 \%$, which lead to $150 \times 150$ vectors in the measurement plane and corresponds to a vector spacing of $3.3 \mathrm{~mm}$ in each direction. For evaluation of the 3C-2D images an interrogation window size of either $96 \times 96$ or $128 \times 96$ pixels was used with an overlap of $75 \%$, resulting in a vector spacing of either $6 \mathrm{~mm}$ or $8 \mathrm{~mm}$. From the instantaneous velocity maps the mean velocity fields $(\bar{u}, \bar{v}, \bar{w})$ and the root mean square $(\mathrm{rms})$ values of the resolved velocity fluctuations were calculated. For a $2 \mathrm{D}$ velocity field, i.e. in our case for $2 \mathrm{C}-2 \mathrm{D}$ PIV results, the rms values were calculated pointwise for $N$ instantaneous velocity fields $\left(v_{\mathrm{i}}, w_{\mathrm{i}}\right)$ by $U_{\mathrm{rms}}=\sqrt{\frac{1}{N} \sum_{i}^{N}\left[\left(v_{\mathrm{i}}-\bar{v}\right)^{2}+\left(w_{\mathrm{i}}-\bar{w}\right)^{2}\right]}$. For the sake of comparability with the velocity fields, we present the rms values rather than the two-dimensional turbulent kinetic energy, which is essentially given by $U_{\text {rms }}^{2}$. When we are talking about "velocity fluctuations" in the following, we refer to the rms values.

\subsection{EXPERIMENTAL RESULTS}

The outline of this section is as follows: First the results for FC and MC, as determined with PIV are presented and discussed (Sections 4.3.1 and 4.3.2). The analysis of the velocity fields by POD is presented in Section 4.3 .3 before in Section 4.3 .4 influences of the buoyancy forces on the flow field are further discussed. In the last Section 4.3.5 the findings from the temperature measurements of the in- and outflowing air are presented. Table 4.2 summarises the measured parameter range.

\subsubsection{ForCED CONVECTION CASE}

Figure 4.3 (a) depicts the time-averaged velocity field of pure FC in the cross section at $X=0.5 \times L$ for $R e=1.07 \times 10^{4}$ and $R a=0(A r=0)$, i.e. with the heating plate switched off and thus $\Delta T \approx 0 \mathrm{~K}$. The velocity vectors are shown for the two intersection lines crossing horizontally and vertically through the rotation centre, which was detected by finding the point where the velocity magnitude equals zero. On the vertical intersection 
TABLE 4.2: Parameter range.

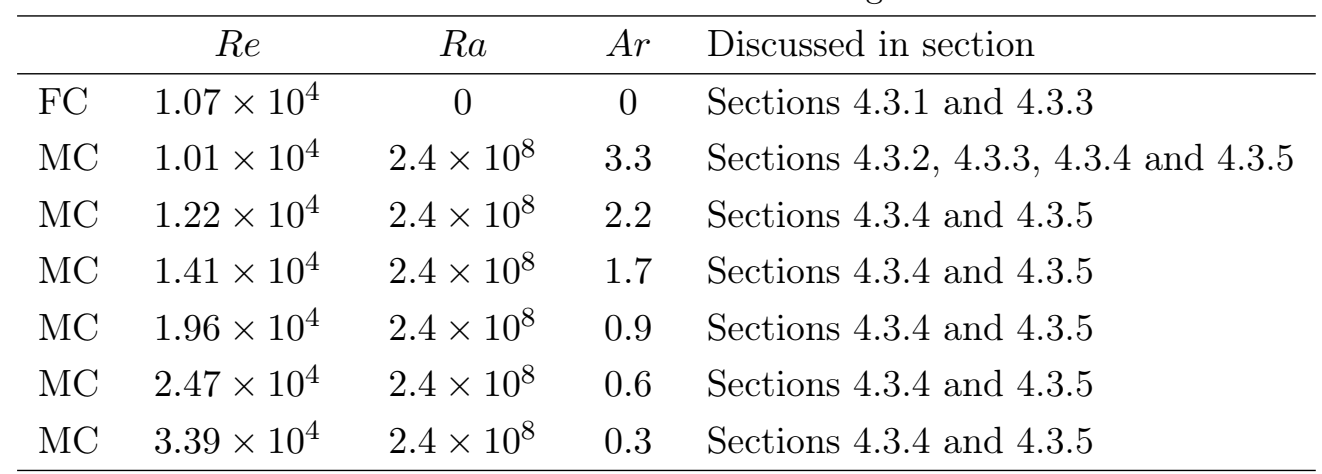

line the wall jet below the ceiling can be clearly detected. It is generated by the incoming air and forced to separate and reattach at the corners of the cell. The jet thus follows the ceiling, the side wall and the floor of the cell. Due to entrainment the average jet velocity decreases and the mass flow rate increases as the jet moves downstream. Therefore only a part of the expanded jet leaves through the outlet while the remaining part recirculates and interacts with the incoming air jet. As a result a counter clockwise rotating mean wind develops in the cell, in good agreement with the CFD results of Saeidi and Khodadadi 92. The corresponding root mean square (rms) values of the resolved velocity fluctuations are presented in Figure 4.3 (b). The rms value in the centre of the jet, which just entered the cell, relative to the mean inflow velocity $v_{\text {in }}$, amounts to $\frac{U_{\text {rms }}}{v_{\text {in }}}<0.04$. This very low value points to a stationary inflow. The shear layer between the incoming air jet and the core region can be identified clearly as locally increased rms values, which reflect the transient jet-bulk interaction caused by the Kelvin-Helmholtz-instability. Further locally increased rms values are found where the jet detaches the ceiling and the left side wall. The lowest absolute velocity fluctuations are located in the bulk region.

In order to further analyse the velocity distribution of the roll structure, line scans of the $w$-velocity component were extracted at $Z=0.5 \times H$. Figure 4.4 (circles, stars and triangles) depicts the line scans for the three measured planes. First it should be noted that the velocity profile is characterised by a constant angular velocity in all three measurement planes. Consequently the mean wind can be approximately described under FC conditions by a two-dimensional solid body rotation (Rankine vortex). The small offset of the line scans in $y$-direction is the result of the additional volume flow in the downward oriented part of the wall jet in the front of the cell.

Figure 4.3 (c) shows the spatial distribution of the $v$-component. In the absence of buoyancy forces the $v$-component depends mainly on the position in $z$-direction (height) while the $u$ and the $w$-components are nearly negligible in this measurement plane. Below the ceiling the air enters the cell trough the air inlet and is directed towards the front window of the convection cell. Above the floor the air leaves the container through the air outlet. A homogeneous flow is observed, which is almost independent of the $x$-coordinate, indicating an almost two-dimensional mean wind. However, a slightly wavy structure 


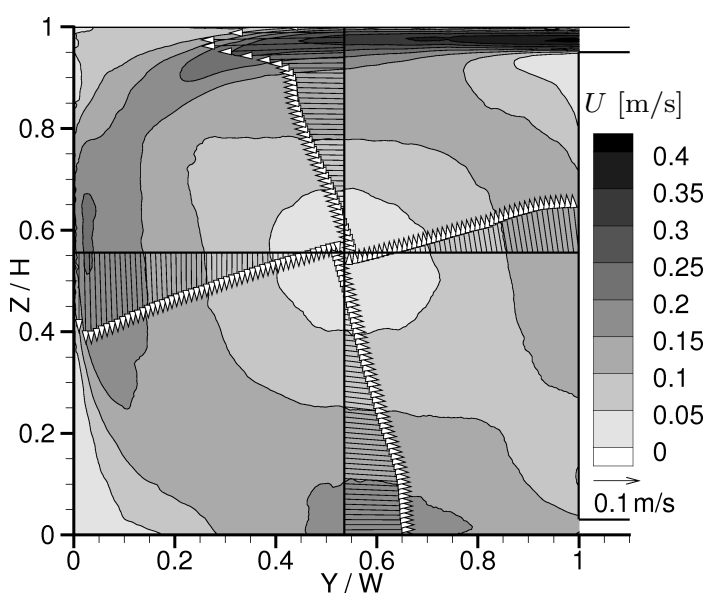

(a)

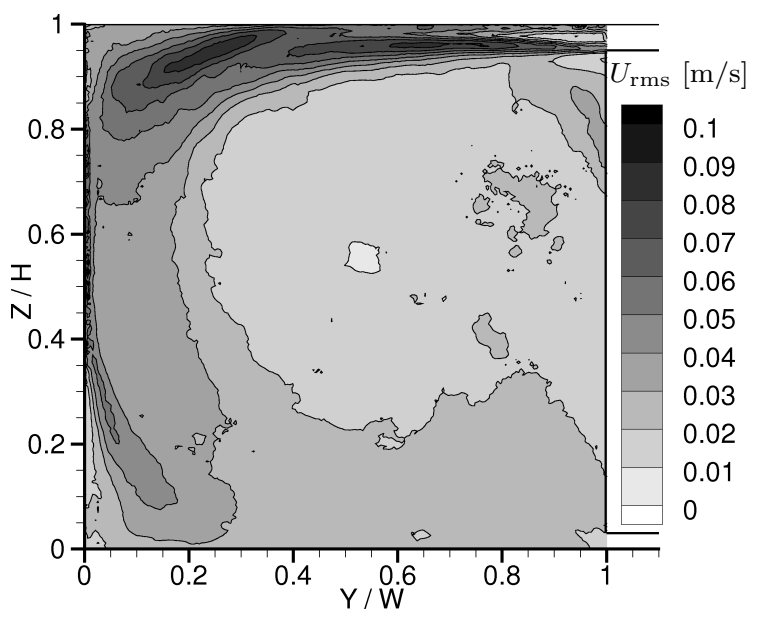

(b)

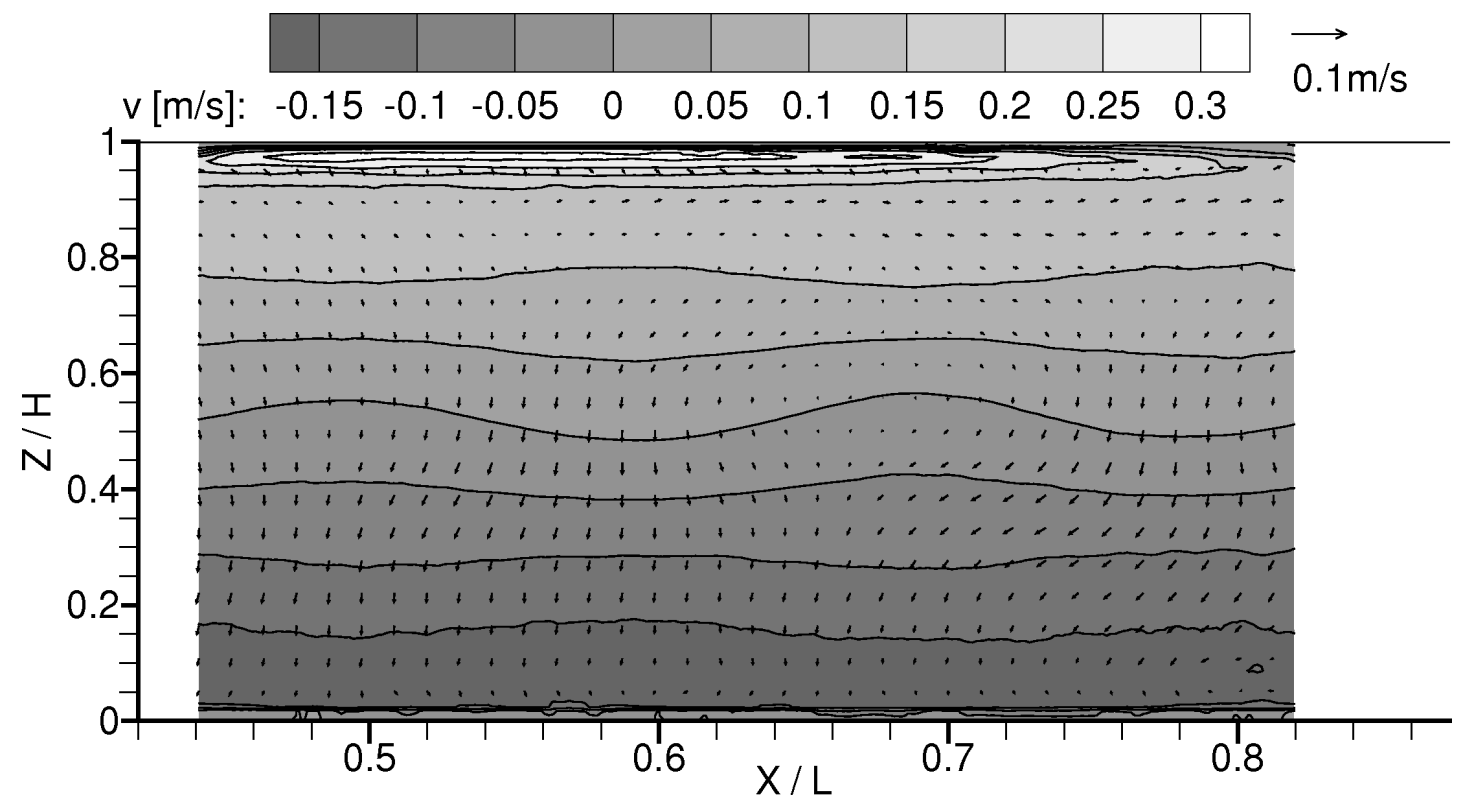

(c)

Figure 4.3: Velocity field and rms-distribution for $R e=1.07 \times 10^{4}$ and $A r=0$. (a) Timeaveraged velocity field in the mid cross section $(X=0.5 \times L)$. The contour shows the velocity magnitude $U=\sqrt{v^{2}+w^{2}}$ and the arrows depict the velocity vectors on the two intersection lines. (b) Rms-values corresponding to Figure 4.3 (a). (c) Time-averaged velocity field in the longitudinal section $(Y=0.5 \times W)$. The contour depicts the $v$-component and the arrows show the $(u \cdot w)$ velocity vectors.

recognisable by the isolines of the $v$-velocity component is detected. The wavy structure has a measured wavelength of $\lambda \approx 500 \mathrm{~mm}$ in longitudinal direction, which matches exactly a value of $\lambda=L \cdot\left(\Gamma_{\mathrm{xz}}\right)^{-1}$. The undulated pattern in the core of the roll structure was also found in tomographic PIV investigations in the same apparatus 65]. As a consequence of the undulation, the centre position of the two-dimensional solid body rotation is not spatially constant in the longitudinal direction. However, the maximal variation in the 
$z$-position in the observed region is less than $\pm 20 \mathrm{~mm}$ in our measurements.

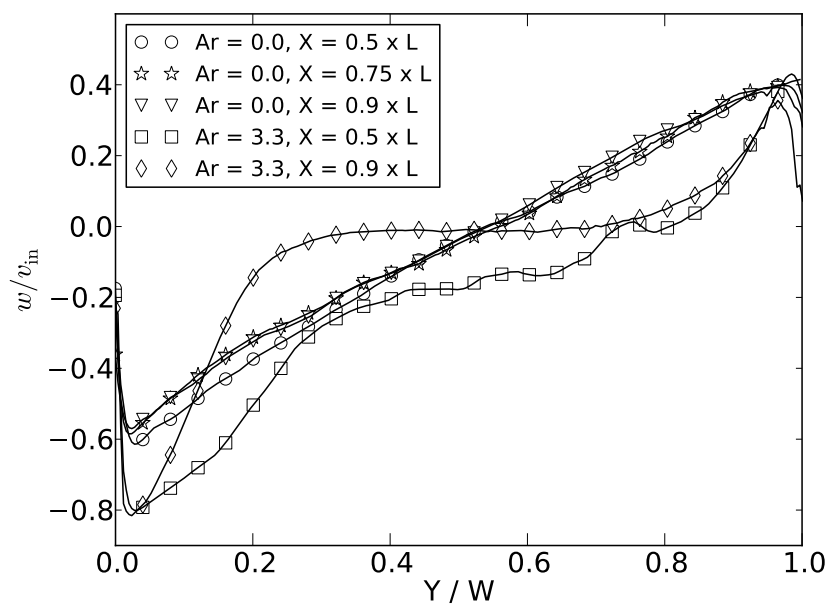

Figure 4.4: Profiles of the $w$-component of the time averaged velocity fields at $Z=0.5 \times H$. For $R e=1.07 \times 10^{4}$ and $A r=0$ profiles at three positions in longitudinal direction (circles, stars and triangles) are plotted. Additional profiles are given for $R e=$ $1.01 \times 10^{4}$ and $A r=3.3$ at two positions in longitudinal direction (squares and diamonds) for comparison.

\subsubsection{MiXed CONVECTION CASE}

The time-averaged velocity field in the vertical cross section at $X=0.5 \times L$ for the MC case ( $R e=1.01 \times 10^{4}, R a=2.4 \times 10^{8}$ and $\left.A r=3.3\right)$ is presented in Figure 4.5 (a). The resulting flow field deviates strongly from that in FC. Again the velocity vectors are shown on two intersection lines crossing horizontally and vertically through the core centre (point of zero velocity). Upon the onset of TC the structure of the wall jet has changed dramatically. As a consequence the core of the mean wind is shifted with respect to the cell centre. The jet separation in the corners of the cell occurs further downstream, which leads to a decrease of the size of the recirculation zones in the corners of the cell as compared to FC. The stationary two-dimensional roll structure detected in FC is disturbed by hot thermal plumes, which are emitted from the thermal boundary layer at the bottom of the cell. Hence, it breaks up and highly three-dimensional and unsteady structures are formed, which are known to reoccur periodically (see [140], where also a detailed analysis of the single velocity components and the dynamics of the centre position of the roll structure are presented). The very small zone of rising air compared to the zone of descending air indicates that in this measurement plane $X=0.5 \times L$ more cold air is sinking from the cooling plate than warm air is rising from the heating plate.

Figure 4.5 (b) depicts the corresponding rms values. As in the FC case, the inflow is rather homogeneous and the shear layer between the incoming air jet and the bulk region produces high velocity fluctuations. However, below the ceiling in the left part of the cell, 


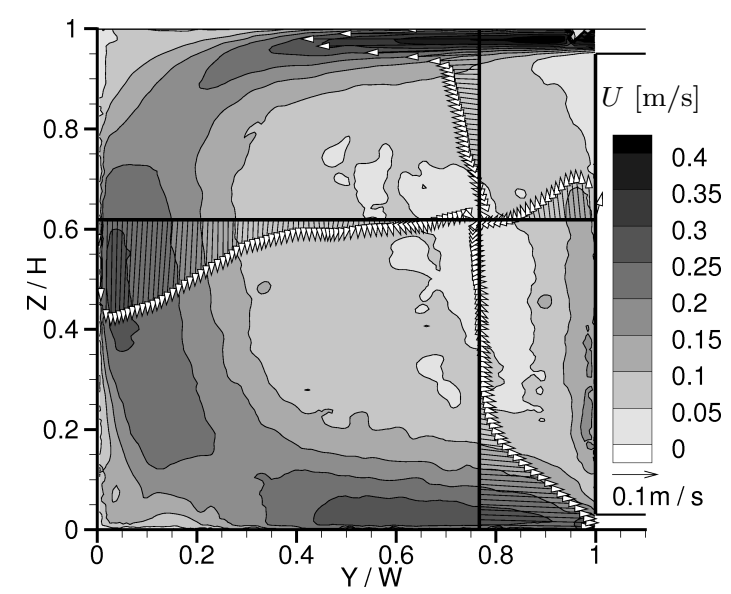

(a)

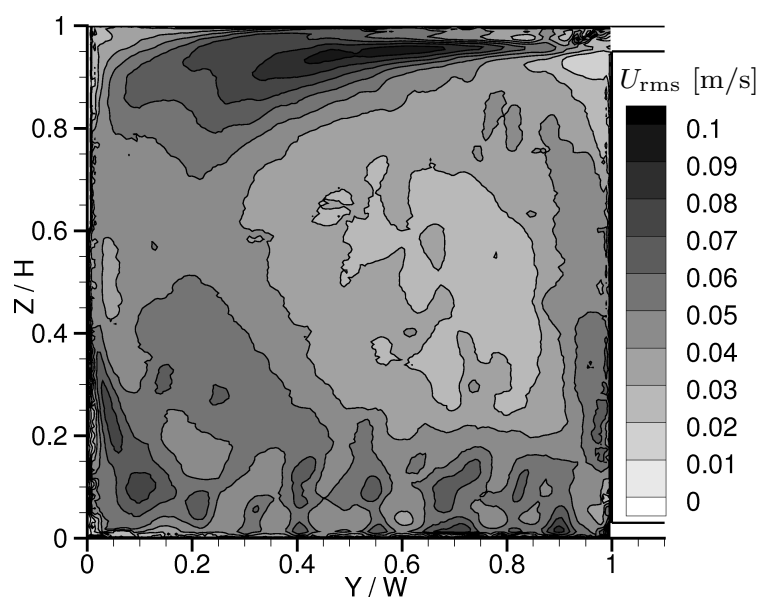

(b)

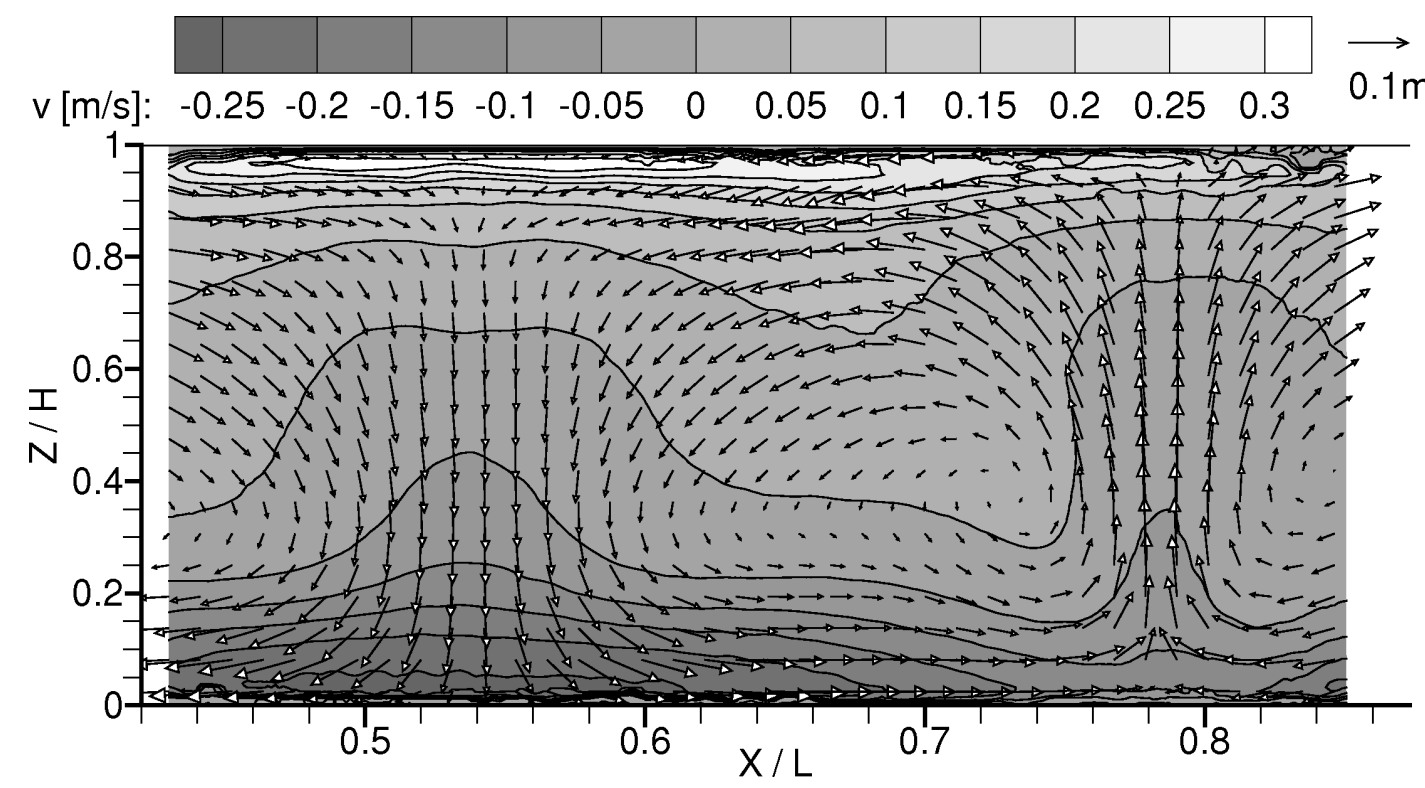

(c)

Figure 4.5: Velocity field and rms-distribution for $R e=1.01 \times 10^{4}$ and $A r=3.3$. (a) Timeaveraged velocity field in the mid cross section $(X=0.5 \times L)$. The contour shows the velocity magnitude $U=\sqrt{v^{2}+w^{2}}$ and the arrows depict the velocity vectors on the two intersection lines. (b) Rms-values corresponding to Figure 4.5 (a). (c) Time-averaged velocity field in the longitudinal section $(Y=0.5 \times W)$. The contour depicts the $v$-component and the arrows show the $(u \cdot w)$ velocity vectors.

there is no region of locally increased rms values, as it is observed for FC. This indicates that there is no fixed, or roughly fixed, separation point at the ceiling anymore. On the left side wall the jet separates at $Z \approx 0.4 \times H$, in almost the same manner as in FC. Further the overall velocity fluctuations are higher as compared to the FC case, indicating a much stronger mixing of the fluid. Very high rms values occur in the lower region next to the heating plate. The locally increased velocity fluctuations are probably caused by localised hot plumes which are emitted from the thermal boundary layer of the heating plate. For a 
further understanding of the threedimensional characteristics of the flow, again the velocity line scans of the $w$-component were extracted at $Z=0.5 \times H$ (see Figure 4.4 , squares and diamonds). Clearly, a breakdown of the solid body rotation is detected. Both, the structure of the wall jet and the location of the mean wind core are different for all cross sections. A strongly three-dimensional nature of the flow can be deduced from the line scans, which deviate considerably for the two investigated measurement planes.

The presence of buoyancy forces leads to a breakdown of the two-dimensional mean wind. More precisely the large-scale flow changes into a superposition of the incoming air jet with smaller flow structures. Figure 4.5 (c) shows the velocity field at $A r=3.3$ in the longitudinal measurement plane. The deviation to the flow field presented in Figure 4.3 (c) pinpoints the influence of buoyancy, i.e. velocity components oriented parallel to the applied temperature gradient establish. Regions of fast upward and downward oriented motion, which are ascribed to the organisation of thermal plumes, are found. The encountered flow field suggests a superposition of the wall jet of incoming air with four buoyancy induced rolls, their rotation axis being oriented mainly in the $y$-direction. This is also reflected in the spatial temperature distribution of the outflowing air: Figure 4.6 depicts the spatially and temporally resolved temperature difference between in- and out flowing air for $A r=3.3$. Thereby, the spatial resolution of approximately $278 \mathrm{~mm}$ and the temporal resolution of approximately $6.15 \mathrm{~s}$ are given by nine temperature sensors, equally spaced in the x-direction over the whole length of the outlet and the scanning frequency, respectively. Two regions, constant in time and space, with increased temperatures are found at $X \approx 0.3 \times L$ and $X \approx 0.75 \times L$, well-fitting the areas of rising hot air inside the cell.

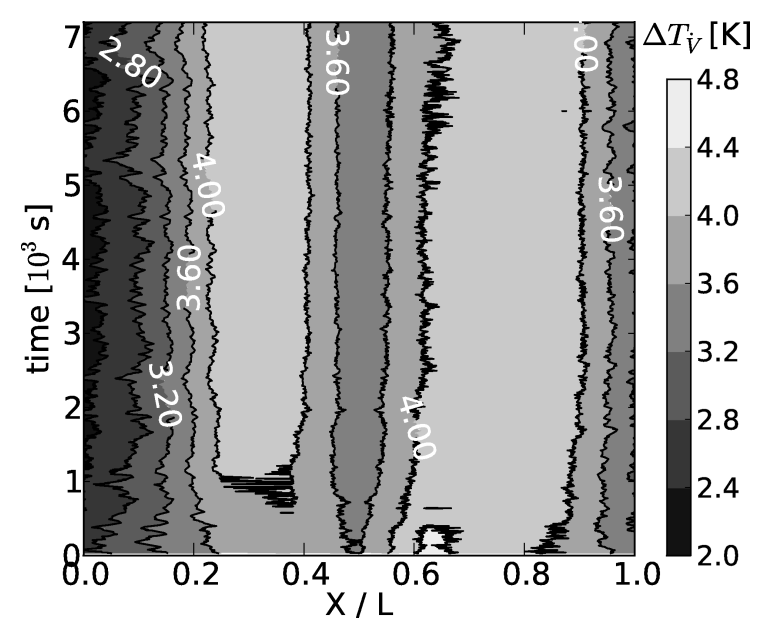

FiguRE 4.6: Distribution of the temperature difference between out- and inflowing air at the end of the air outlet as a function of time and $x$-direction. Measured for $R e=1.01 \times 10^{4}$ and $A r=3.3$. 


\subsubsection{PROPER ORTHOGONAL DECOMPOSITION}

To identify the coherent large-scale flow structures, a Proper Orthogonal Decomposition (POD) of the 3C-2D velocity fields has been performed by applying the snapshot method given by Sirovich 112,113. For further information on the POD calculation the reader is referred to Westhoff et al. [140].

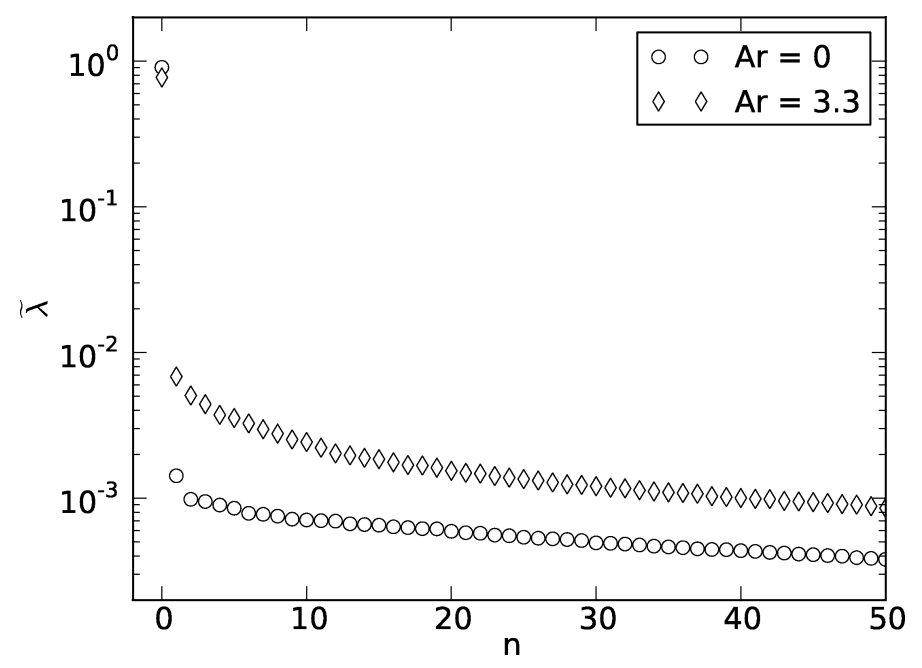

Figure 4.7: Normalised energy eigenvalues $\tilde{\lambda}$ of the first 50 modes of the POD calculated for the three dimensional velocity fields in the longitudinal cross section at $Y=0.5 \times W$ obtained for $A r=0$ and $A r=3.3$.

In Figure 4.7 the normalised energy eigenvalues $\tilde{\lambda}$ of the first 50 modes of the POD of the three-dimensional velocity fields analysed in the longitudinal cross section $Y=0.5 \times W$ are presented. The eigenvalues are listed in descending order. Thereby, $\tilde{\lambda}$ is a measure for the energy of the corresponding mode with respect to the total energy. The eigenvalues are normalised by the sum of the first 800 modes: $\sum_{n=1}^{800} \tilde{\lambda}_{n} \equiv 1$.

Prior to the discussion of the POD results, it should be emphasised that the magnitude of the eigenfunctions $\Phi_{n}=\sqrt{\phi_{n, u}^{2}+\phi_{n, v}^{2}+\phi_{n, w}^{2}}$ denotes what we call a coherent structure in the following. Here, $n$ denotes the mode number and $\phi_{n}$ represents the eigenfunctions of the corresponding velocity component. For a reasonable comparison of the coherent structures $\Phi_{n}$ is normalised by the maximum value $\max \left(\Phi_{n}\right)$.

In the FC case the first mode contains nearly $91 \%$ of the total energy. Any of the other modes carry less than $1 \%$. In very good agreement with this result, the comparison of the topology of the first coherent structure (Figure 4.8) with the time-averaged velocity field (Figure 4.3 , c) reveals that both are nearly congruent. From this we conclude that the other coherent structures represent smaller fluctuations, which are primarily found at the ceiling of the cell, where the wall jet oscillates due to the Kelvin-Helmholtz-instability. Moreover, the high energy of the first mode underlines that the flow in the pure FC case is very steady. Comparing the eigenvalue distribution of the MC case with the FC case it can be seen that still a coherent structure exists, which contains most of the energy, i.e. $\tilde{\lambda}_{1}=77 \%$. However, compared to the FC case $\tilde{\lambda}_{1}$ has decreased significantly. Moreover 


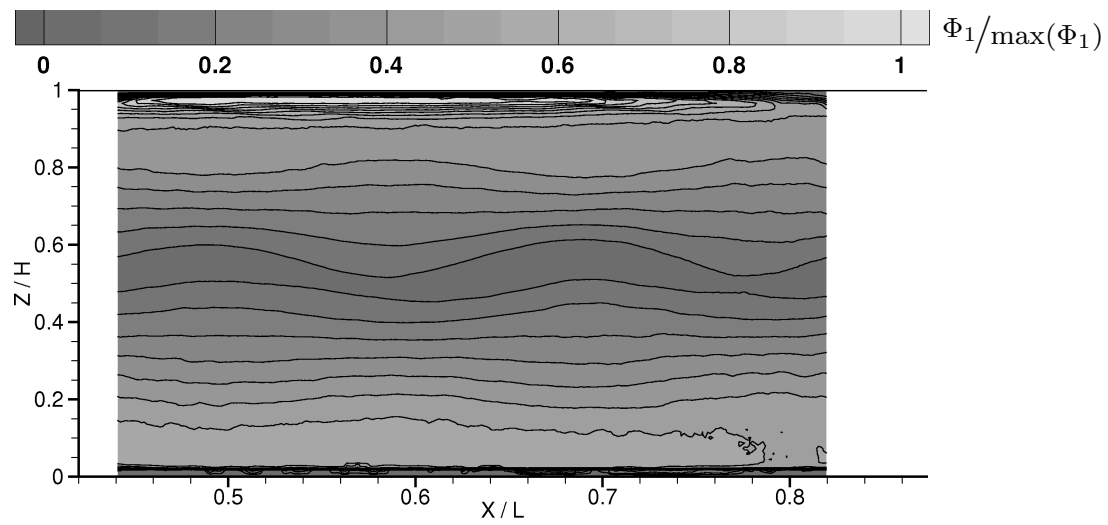

FiguRE 4.8: Topology of the coherent structure $\Phi_{1}$ of the 1 st mode obtained for $R e=1.07 \times 10^{4}$ and $A r=0$.

additional modes with eigenvalues of $\tilde{\lambda}>1 \%$ are found; for example the second mode still contains nearly $7 \%$ of the total energy. Hence, it is summarised that the onset of a buoyancy induced secondary flow leads to an decrease of the first mode energy $\tilde{\lambda}_{1}$, as well as an increased number of modes containing $\tilde{\lambda}>1 \%$ of the total energy.

In the following the first, second, fifth and sixth POD mode are analysed for the MC case. First it should be noted, that the sixth mode still contains as much as $3.4 \%$ of the total energy. In Figure 4.9 (a) the coherent structure of the first mode is shown. Similar as in the FC case (see Figure 4.8) the topology of $\Phi_{1}$ for MC is nearly congruent with the corresponding time-averaged velocity field. We conclude from this, that this mode represents the buoyancy induced large-scale circulation in longitudinal cell direction. The coherent structure, which belongs to the 2nd mode shown in Figure 4.9 (b), indicates elevated values where hot plumes are emitted from the bottom thermal boundary layer. Moreover, the structure of this mode reflects the splitting of the plumes to the left or right shortly after the emission of the plume from the thermal boundary layer. This separation of the plume is also revealed in Figure 4.9 (c) where the topology of $\Phi_{5}$ is shown. But in contrast to $\Phi_{2}$ the flow is divided into the left or right direction at $Z \approx 0.5 \times H$. The two coherent structures $\Phi_{3}$ and $\Phi_{4}$ are found to depict mainly the same topology as $\Phi_{2}$, therefore they are not shown for the sake of brevity. Locally increased $\Phi_{6}$ values are found in the region of falling plumes in Figure 4.9 (d). Thus we associate this mode to the descending cold air in the centre region of the container. However, in contrast to the 2 nd mode, a clear location for plume emission cannot be detected. The reason for this might be the interaction of the detaching wall jet at the ceiling of the container, which leads to an emission of cold plumes in a wider region in the boundary layer at the ceiling.

\subsubsection{INCREASING BUOYANCY FORCES}

In the previous subsection only the two flow cases $A r=0$ and $A r=3.3$ have been discussed. In this subsection the influence of increasing buoyancy forces on the forced flow is determined as a function of $A r$. Thereby, the Archimedes number was increased by decreasing the 

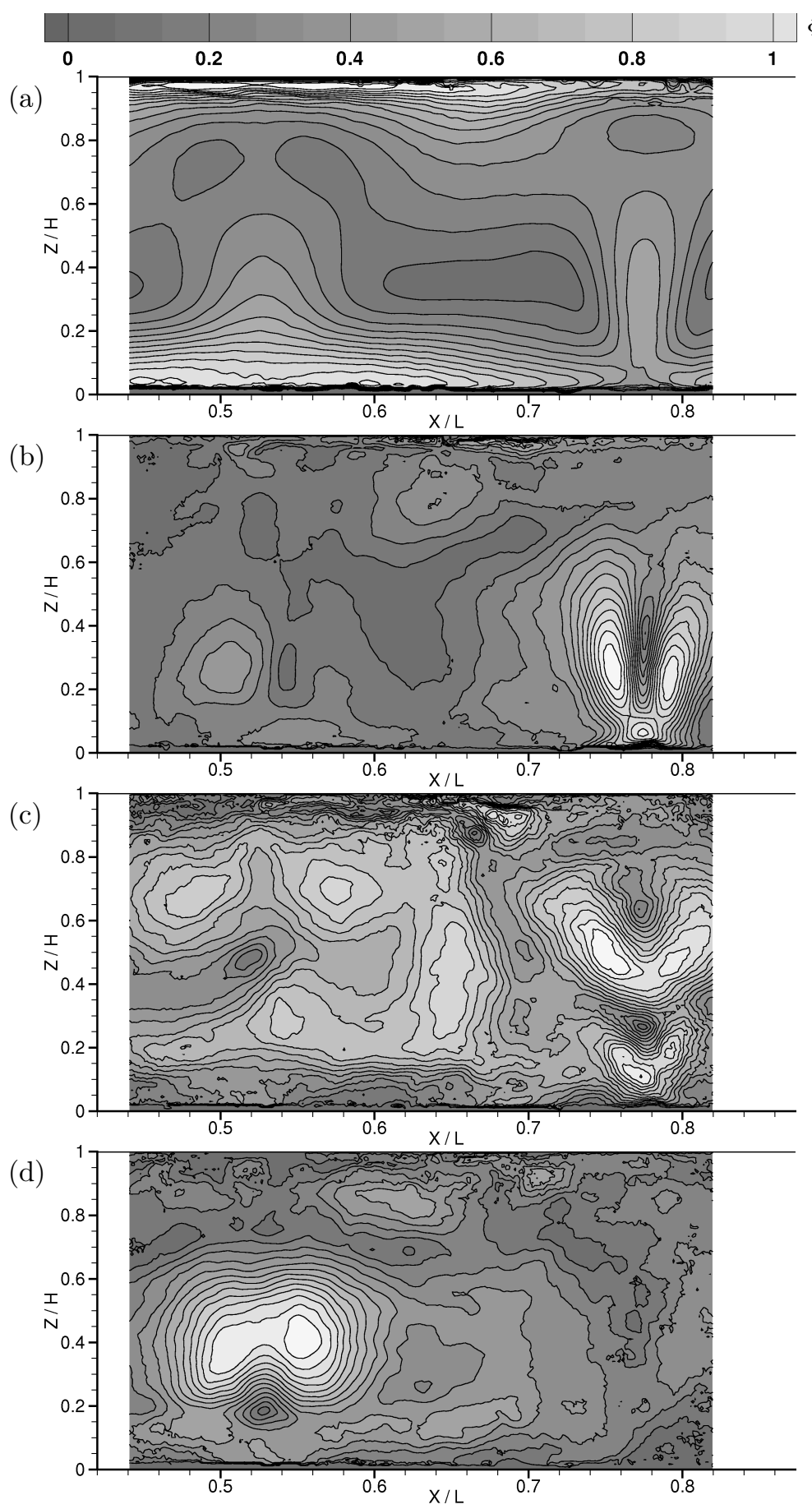

Figure 4.9: Topology of the coherent structures $\Phi_{\mathrm{n}}$, (a) 1st mode, (b) 2nd mode, (c) 5th mode and (d) 6th mode obtained for $R e=1.01 \times 10^{4}$ and $A r=3.3$. The legend in the top applies to all plots.

inflow velocity for a constant temperature difference between top and bottom of the cell, which leads to a variation of $A r$ by varying $R e$ for fixed $R a$. In order to study the growing influence of buoyancy forces on the flow with rising $A r$ the normalised difference of the $w$-component of the velocity are presented in Figure 4.10 (a) for MC and FC along the 
intersection line $Z=0.1 \times H$ in the $X=0.5 \times L$ plane. Thereby, the difference of the $w$-component between $\mathrm{MC}$ and $\mathrm{FC}$ is normalised by the the mean inflow velocity. Thus, $\Delta w=\frac{w_{\mathrm{MC}}-w_{\mathrm{FC}}}{v_{\text {in }}} \approx 0$ indicates that the effect of buoyancy is negligible. The increase of $\Delta w$ with increasing $A r$ indicates the growing modulation of the flow by the buoyancy forces.

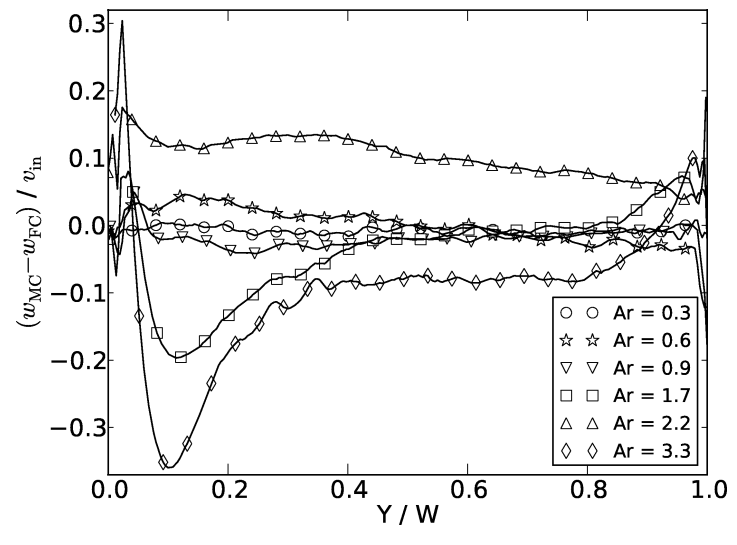

(a)

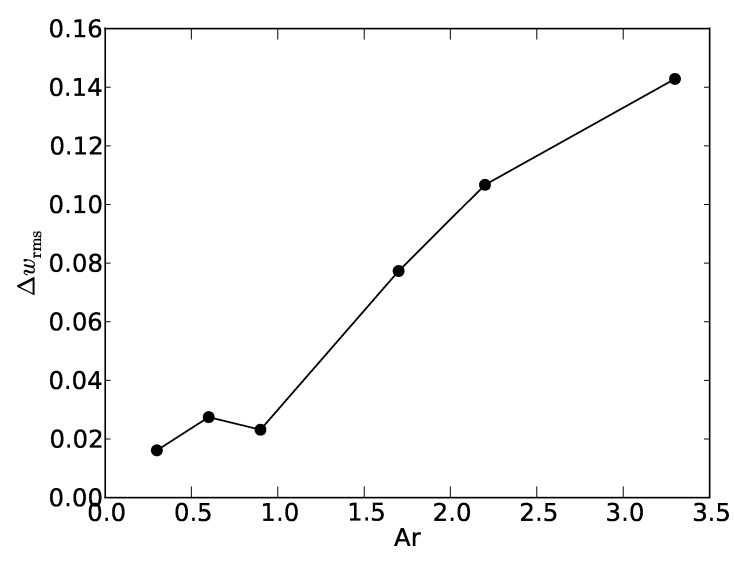

(b)

Figure 4.10: (a) $w$-component of the velocity for MC minus $w$-component of the velocity for FC normalised with the mean inflow velocity for various $A r$ at $X=0.5 \times L$ and $Z=0.1 \times H$. (b) $\Delta w_{\mathrm{rms}}$ as function of $A r$.

In addition the rms values of $\Delta w$ were calculated by $\Delta w_{\mathrm{rms}}=\sqrt{\frac{1}{N} \sum_{\mathrm{i}}^{N}\left(\frac{w_{\mathrm{MC}, \mathrm{i}}-w_{\mathrm{FC}, \mathrm{i}}}{v_{\mathrm{in}}}\right)^{2}}$, where $N$ denotes the number of data points. The rms values are a measure for the difference between MC and FC and in this respect reflect the relevance of buoyancy for the flow.

For the smallest Archimedes number, $A r=0.3, \mathrm{rms}$ values as low as $\Delta w_{\text {rms }}(A r=0.3)=0.017$ are found, indicating that the influence of the buoyancy forces on the flow field is negligible. For $A r=0.6$ and $A r=0.9$ the rms values are still less than $\Delta w_{\text {rms }}=0.03$ but the impact of buoyancy is already growing. With rising $A r$ the rms values increase up to $\Delta w_{\text {rms }}(A r=3.3)=0.14$ due to the influence of buoyancy on the $w$-component on the mean flow field. We further found that the sense of rotation of the flow structures (see Section 4.3.2) alternates with rising $A r$, see Figure 4.10(a). For $A r=1.7$ and $A r=3.3$ the TC induces a downdraught in the observed measurement plane $(X=0.5 \times L)$. For $A r=3.3$ this downdraught leads to four convection rolls whose axis are orientated in the longitudinal cell direction. In these convection rolls, which were also detected in the temperature profile of the outflowing air (Figure 4.6), fluid falls at $X \approx 0 \times L, X \approx 0.5 \times L$ as well as $X \approx 1 \times L$ and rises at $X \approx 0.25 \times L$ as well as $X \approx 0.75 \times L$, see Figure 4.11 (b) and Figure 4.10 (a). The spatial temperature distribution in the outflowing air, presented in Figure 4.6. matches the above discussed orientation of the rolls for this $A r$, since warmer and colder regions are found in areas of rising warm and descending cold air, respectively. For $A r=2.2$, on the other hand, an updraught in the observed measurement plane is found, which reflects the change of sense of rotation. Thus, at $A r=2.2$ the convection rolls induce updraughts in the vicinity of the sides as well as in the centre region of the 
convection cell and downdraughts at $X \approx 0.25 \times L$ as well as $X \approx 0.75 \times L$, see Figure 4.11 (a) and Figure 4.10 (a). By extrapolating the results of the stereoscopic PIV data to the full length of the cell, the two convection rolls in the measurement section confirm the existence of these four convection rolls as well as their alternation, see Figure 4.11. The maximal velocity magnitude in the $x-z$ plane is $\{u, w\}_{\max }=0.23 \times v_{\text {in }}$ and $\{u, w\}_{\max }=0.41 \times v_{\text {in }}$ at $A r=2.2$ and $A r=3.3$, respectively, clearly indicating the increasing importance of the buoyancy induced roll structures for the resulting flow field.

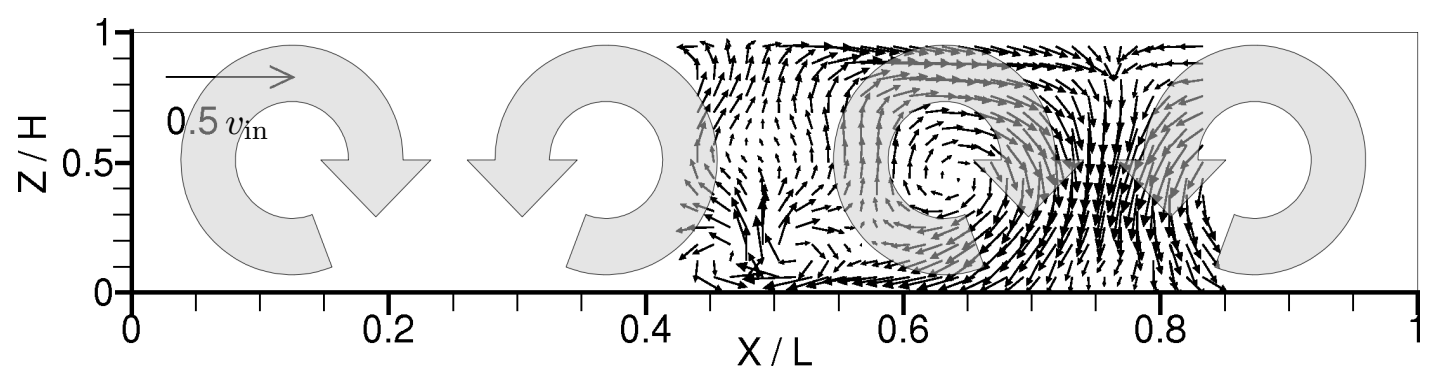

(a)

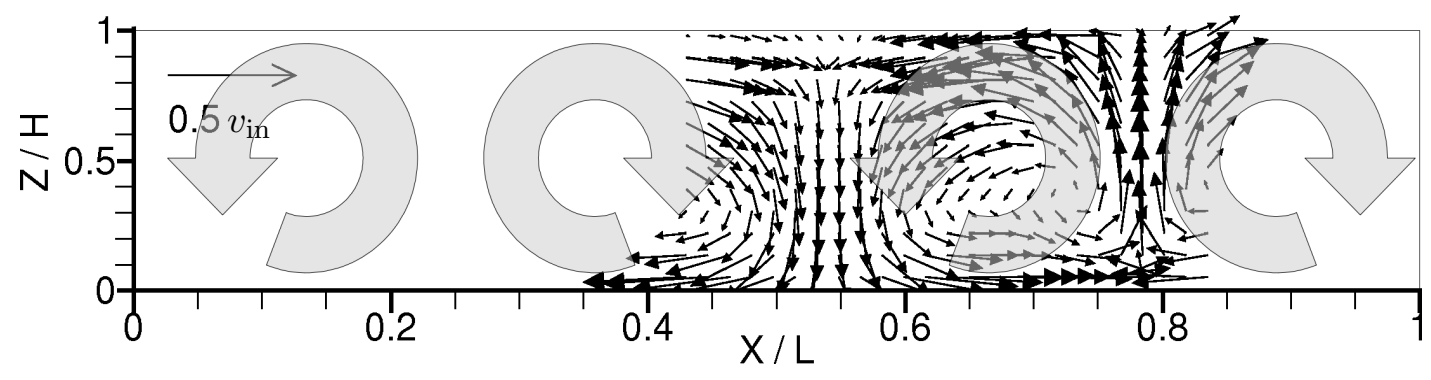

(b)

FIGURE 4.11: Sketch of the additional convection rolls superimposed with mean velocity vectors in the $x-y$ plane normalised with the corresponding mean inflow velocity. (a) $R e=1.2 \times 10^{4}$ and $A r=2.2$. (b) $R e=1.01 \times 10^{4}$ and $A r=3.3$.

Tomographic PIV measurements of Kühn et al. 65 in the same apparatus showed that these rolls are not oriented parallel to the shorter side of the cell, but rather reflect a $\mathrm{W}$-shape in the $x-z$ plane.

\subsubsection{TEMPERATURE MEASUREMENTS AND ENTHALPY FLUX}

The formation and the dynamics of the large-scale structures govern the heat transport through the cell, reflected in the characteristic temperature distribution, which was observed at the cell outlet, see Figure 4.6. In order to shine some light on the relationship between large-scale flow structure and enthalpy flux, the normalised time-averaged temperature difference between out- and inflowing air is presented for the investigated $A r$ in Figure 4.12 (a). While a clear dependence of its amplitude on $A r$ can be observed, the spatial distribution of the normalised temperature difference with two maxima does not seem to depend upon $A r$. The time resolved plot of this temperature difference, as depicted in Figure 
4.6. shows that the distribution is also constant in time, at least on time scales of the order of minutes or larger. It should be noted that the change of sense of rotation for $A r=2.2$ compared to $A r=3.3$ and the independent spatial temperature distribution indicate that the structure of the large-scale circulations in the cell centre, i.e. at $Y=0.5 \times W$, does not necessarily determine the temperature distribution at the end of the outlet channel. Otherwise the positions of the maxima in the temperature distribution would change their position with the different positions of the rising hot air. This conflicting effect seems to be a result of the fact that the flow is rather threedimensional, thus the flow field in the layer close to the outlet, i.e. at $Y \approx 1 \times W$, which determines the temperature profile at the end of the outlet channel, might be different compared to the centre cross section, i.e. $Y=0.5 \times W$. The accordance of the temperature profile and the measured flow field for $A r=3.3$ reflects, that for this $A r$ the four additional roll structures are rather stationary in the cell. These additional buoyancy induced rolls are not aligned perfectly parallel, but rather in a $W$-shape as observed in tomographic PIV measurements of Kühn et al. 65. Nevertheless, areas in $x$-direction in which the fluid rises in $Y \approx 1 \times W$ seem to be the same as in the centre cross section, i.e. at $Y=0.5 \times W$. In contrast, for $A r=2.2$, the mismatch of temperature and flow field indicates that the buoyancy induced secondary flow generates roll structures with different areas of rising hot fluid in $x$-direction for different $Y$ cross sections. Thus, to relate the temperature distribution to the large-scale circulation a consideration of the whole three-dimensional flow field would be necessary.

To investigate the dependence of the absolute temperature differences on $A r$ in more detail the enthalpy flux, which develops since warmer fluid is leaving and colder fluid is entering the cell, is analysed. Thereby, the enthalpy flux of the fluid $\left(h_{\mathrm{B}}-h_{\mathrm{A}}\right.$, see Figure 4.12 b), which can be interpreted as the amount of convected heat leaving the cell per time unit, is given by $\frac{\Delta Q}{\Delta t}=c_{\mathrm{p}} \cdot \rho \cdot \dot{V} \cdot \Delta T_{\dot{V}}$, where $c_{\mathrm{p}}$ denotes the specific heat capacity at constant pressure, $\rho$ the density, $\dot{V}$ the volume flow rate and $\Delta T_{\dot{V}}$ the temperature difference between out- and inflowing air.

In Figure 4.12 (b) the contributions to the enthalpy balance of the cell are sketched, where enthalpy fluxes are symbolised by the grey arrows $\left(h_{\mathrm{A}}\right.$ to $\left.h_{\mathrm{E}}\right)$. Here $h_{\mathrm{A}}$ and $h_{\mathrm{B}}$ denote the enthalpy fluxes due to the inflowing and outflowing air, respectively, $h_{\mathrm{C}}$ is the thermal energy flux dissipating at the ceiling of the cell and $h_{\mathrm{D}}$ is the thermal energy flux, induced by the heating plate. Enthalpy fluxes through the sidewalls of the cell $\left(h_{\mathrm{E}}\right)$ can either point into or out of the cell, depending on the ambient conditions. Since double glazing was used for the side walls with an air gap in-between and since the temperature of the surrounding air is nearly equal to the mean fluid temperature in the cell, the gross enthalpy fluxes through the side walls $\left(h_{\mathrm{E}}\right)$ can be neglected. The flux $h_{\mathrm{D}}$ of the heating plate must equal zero in all $\mathrm{FC}$ cases, because the heating plate is switched off and thermally insulated against the surrounding. For the $\mathrm{MC}$ cases a constant temperature difference between the heating and the cooling plate of $\Delta T_{\mathrm{HC}}=21.2 \mathrm{~K}$ is realised. The ceiling of the cell is kept at ambient temperature by a cooling plate. Since in the FC dominated cases the temperature of the inflowing air is nearly the same as the ambient air temperature, the enthalpy flux $h_{\mathrm{C}}$ 


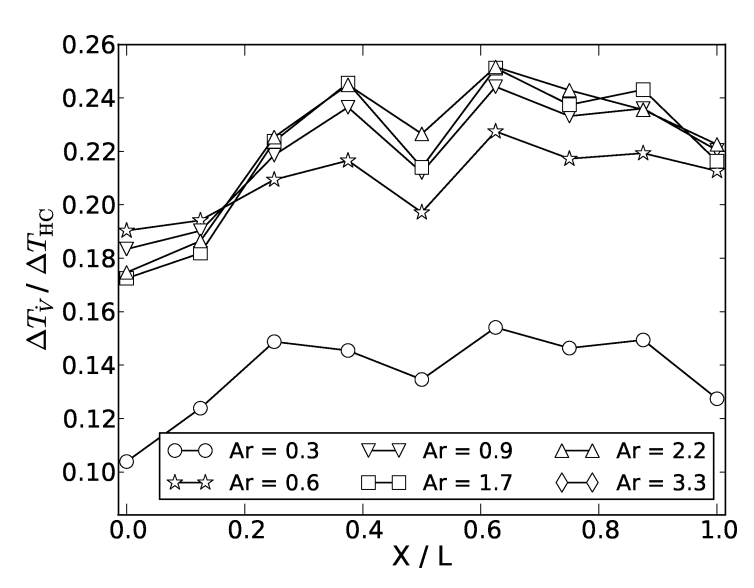

(a)

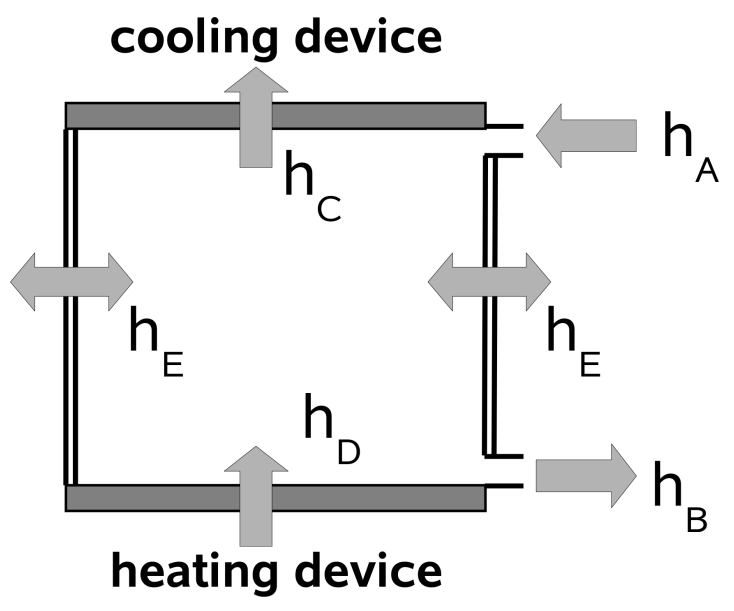

(b)

FiguRE 4.12: (a) Normalised temperature distribution of the outflowing air. (b) Sketch of the enthalpy balance of the cell with the denoted enthalpy fluxes.

is negligible too. For $A r<1$, the flow is still dominated by FC. As a result the momentum of the hot rising air originating from the bottom thermal boundary layer is much smaller than the inertia forces, and the hot plumes are swallowed directly by the air flow leaving the cell through the outlet at the bottom. Therefore, the dissipation of thermal energy through the ceiling $\left(h_{C}\right)$ is considered to be still small in this case. For higher $A r$ buoyancy effects become more important and more enthalpy is transported from the heated bottom to the ceiling by thermal plumes. Thus, the enthalpy flux $h_{\mathrm{C}}$ is expected to increase with $A r$. In order to minimise disturbances of the flow in the cell, which could be induced by the temperature sensors, the temperature of the outflowing air has been measured at the end of the outlet channel. In order to determine the temperatures at the outlet of the cell, we use an empiric equation [4] to estimate the channel Nusselt number and to take into account the heat loss of the outflowing air while passing the outflow channel. In Figure 4.13 (left axis, squares) the temperature difference between outflowing and inflowing air averaged in space and time $\Delta T_{\dot{V}}$ is shown for different $A r$. It is normalised with the mean temperature difference between the heating and cooling plates $\left(\Delta T_{\mathrm{HC}}=21.2 \mathrm{~K}\right)$. For $A r<0.9 \Delta T_{\dot{V}}$ first increases steeply with $A r$ but saturates very soon above $A r \approx 1$. The saturation of $\Delta T_{\dot{V}}$ above that value indicates that the heat flux through the cell is governed by buoyancy forces in this range of $A r$, while inertia forces, if at all, play only a minor role. As a consequence a change of $A r$, and thus $R e$, will not alter $\Delta T_{\dot{V}}$ in this range. This finding is in good agreement with Figure 4.10 (b), which reveals a plateau-like behaviour of $\Delta w_{\text {rms }}$ for $A r<1$ and a steep increase of $\Delta w_{\text {rms }}$ for $A r>1$.

Results of the normalised enthalpy flux $\left(\left.\frac{\Delta Q}{\Delta t}\right|_{A r \approx 1} \equiv 1\right)$ are presented in Figure 4.13 (right axis, circles). Above $A r \approx 0.6$ the enthalpy flux decreases with rising $A r$ since first $\Delta T_{\dot{V}}$ saturates for high $A r$, as discussed above, and second since the volume flow rate drops down with increasing $A r$. Below $A r \approx 0.6$ the enthalpy flux rises with increasing $A r$, since 


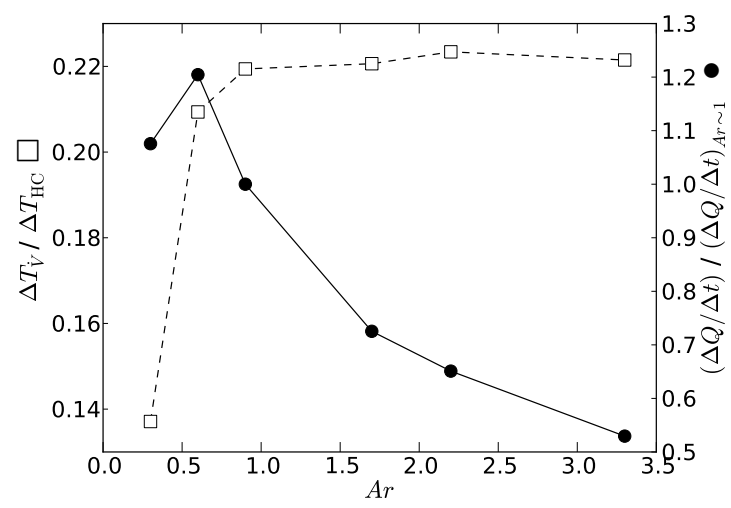

Figure 4.13: Left axis, $\square$ : Temperature difference between out- and inflowing air averaged in space and time for different $A r$, and normalised with the mean temperature difference between heating and cooling plate. Right axis, $\bullet$ : Enthalpy flux averaged in space and time for different $A r$, normalised with the averaged enthalpy flux at $A r \approx 1$.

the temperature difference grows, until the maximum is reached for $A r \approx 0.6$. The fact that the temperature difference decreases with rising $R e$, i.e. with decreasing $A r$, below $A r \approx 0.6$ can be understood as follows: On the one hand the enthalpy flux between heating plate and fluid, $h_{\mathrm{D}}$, grows with increasing fluid velocity, and thus with decreasing $A r$. On the other hand, since lower $A r$ are generated by rising $R e$, this is accompanied by a higher entrainment of fluid from the cell centre by the wall jet. According to the velocity line scans in Figure 4.3 (a) and Figure 4.5 (a), entrainment occurs mainly close to the cooling plate. This results in a higher heat transfer $h_{\mathrm{C}}$ between cell core and cooling plate. However, due to the higher shear at the cooling plate as compared to the heating plate the latter of the two processes dominates and as a result the normalised enthalpy flux decreases with decreasing $A r$ in this range.

Two important observations have been made with these temperature measurements: The first is the existence of an upper boundary for $\Delta T_{\dot{V}}$. The temperature difference first increases with increasing $A r$, then approaches a constant value of $\Delta T_{\dot{V}} \approx 4.6 \mathrm{~K}$ for $A r>0.9$. The second is the existence of a maximum in the enthalpy flux of the fluid at $A r \approx 0.6$. As a consequence there are certain flow conditions which maximise the enthalpy flux of the flow through the cell.

\subsection{Conclusion}

FC and $\mathrm{MC}$ has been investigated under controlled conditions in a rectangular convection cell with air as working fluid $(\operatorname{Pr} \approx 0.72)$. Two flow cases have been studied in detail: Pure FC for $R e=1.07 \times 10^{4}$ and $R a=0(A r=0)$ and $\mathrm{MC}$ for $R e=1.01 \times 10^{4}$ and $R a=2.4 \times 10^{8}(A r=3.3)$. For all cases $2 \mathrm{C}-2 \mathrm{D}$ and $3 \mathrm{C}-2 \mathrm{D}$ PIV has been conducted in order to study the flow in three cross sectional and one longitudinal measurement plane. The 
resulting velocity fields and the resolved velocity fluctuations reveal a strong dependence on the ratio of inertia and buoyancy forces. For pure FC the air flow in the cell is governed by a nearly two-dimensional solid body like rotation with separation regions in the cell corners. Upon the onset of TC, i.e. for $A r=3.3$ a collapse of the $2 \mathrm{D}$ mean wind was observed, as well as a formation of four convection rolls, which interact with the wall jet generated by the inflowing air. A POD of the instantaneous velocity fields confirms the existence of these large-scale coherent structures. Moreover, the POD results show that the onset of a buoyancy induced secondary flow leads to a decrease of the first mode energy as well as an increased number of modes containing more than $1 \%$ of the total energy. The observed flow structures are also visible in the temperature series measured in the air outlet. Thereby, these temperature distributions with two maxima over the length of the outlet are constant in time and space. A further result of the temperature measurements is the existence of an upper boundary for the temperature difference between out- and inflowing air. The temperature difference first increases with increasing $A r$ and then approaches a constant value for $A r>0.9$. This saturation indicates that the heat flux through the cell is governed by buoyancy forces in this range of $A r$, while inertia forces, if at all, play only a minor role. As a consequence a change of $A r$, and thus $R e$, will not alter the temperature difference in this range. This finding is in good agreement with the observed plateau-like behaviour of rms-values of the $w$-component for $A r<1$ and its steep increase for $A r>1$. Finally, as a result of the upper boundary for the temperature difference between out- and inflowing air and the decreasing $R e$ for increasing $A r$, a maximum in the enthalpy flux of the fluid is found at $A r \approx 0.6$. 



\title{
5 Temperature OsCillations IN TURBULENT MC [102]
}

Citation and credit: Reprinted with permission from Turbulence and Interactions, Proceedings of the TI 2012 conference, in volume 125 of Notes on Numerical Fluid Mechanics and Multidisciplinary Design, pages 157-163, 2014, DOI:10.1007/978-3-662-43489-5_19, Copyright 2014, Springer-Verlag Berlin Heidelberg

Reference: 102

Title: "Temperature Oscillations in Turbulent Mixed Convective Air Flows"

Authors: Daniel Schmeling, Johannes Bosbach and Claus Wagner

Contributions: I performed all measurements, analysed them and made all figures. I wrote the first draft, whose structure has been preserved.

\begin{abstract}
We report on measurements of temperature oscillations observed in turbulent mixed convection in a rectangular enclosure. They are correlated to fluctuations and spontaneous reorientations of the large-scale circulations in the flow. Stable temperature configurations, periodic reconfigurations as well as spontaneous reversals and reorientations were detected. We ascribe the observed dynamics of the large-scale flow to the interaction between the superimposed shear forces of the forced convective flow and the buoyancy driven flow. Comparison with smoke visualisations allowed to assign the observed temperature distributions to a certain number and configuration of convection rolls. A frequency analysis of the oscillations as a function of the characteristic numbers is conducted revealing a sophisticated dependency of the dynamics of the large-scale flow on $A r, R e$ or $R a$.
\end{abstract}




\subsection{INTRODUCTION}

Mixed convection (MC) denotes a flow in which forced convection, caused by inertia forces, and thermal convection, initiated by buoyancy forces, are of the same order of magnitude. In MC, the Archimedes number $A r=R a \times R e^{-2} \times P r^{-1}$ serves as a measure for the ratio between thermal and inertia forces. Here, $R a$ denotes the Rayleigh number with the height of the cell $H$ and the temperature difference $\Delta T$ between bottom and ceiling as characteristic length and temperature difference, respectively. For the Reynolds number $R e$, the mean inflow velocity $u$ and again the height of the cell serve as characteristic values while the Prandtl number $\operatorname{Pr}$ merely depends on the fluid material properties.

Previous investigations of the flow in the rectangular convection cell addressed structure formation, heat transport and low-frequency oscillations [66, 105,140$]$. Thereby, temperature oscillations in the out-flowing air, the arrangement of large-scale circulations (LSCs) and oscillations of time development coefficients of Proper Orthogonal Decomposition modes of the velocity fields were analysed. The details of the underlying dynamics of the LSCs, which govern these effects, however, are still open issues. In pure Rayleigh-Bénard convection $(\mathrm{RBC})$ in a similar rectangular cell with aspect ratio five, the arrangement and the dynamics of the LSCs 106 were studied for $10^{7}<R a<10^{10}$ by means of Large Eddy Simulations (LES). In their study they observed two, three and four roll configurations. Dynamical effects like cessations or spontaneous reorientations of the LSC are well known from turbulent $\mathrm{RBC}$ in cylindrical geometries [20]. Thereby, the aspect ratio strongly affects the azimuthal motion, reorientation, cessation and reversals of the LSC [148]. Besides the detailed studies on the dynamics of the LSCs in pure RBC, little is known about the effects in systems for which thermally induced LSCs are interacting with externally driven shear flows.

\subsection{EXPERIMENTAL SETUP AND MEASUREMENT TECHNIQUES}

All investigations presented below were conducted in a rectangular convection cell with a length of $L=2500 \mathrm{~mm}$ and aspect ratios of $\Gamma_{x z}=L / H=5$ and $\Gamma_{y z}=W / H=1$, see Fig. 5.1. In order to accomplish nearly isothermal boundary conditions the heating device at the bottom consists of five separated sub-plates, each of them being actively heated via a temperature controlled water circuit. The ceiling is passively cooled close to ambient temperature via an air-air heat exchanger.

This configuration provides isothermal boundary conditions at the bottom and ceiling with maximal spatial temperature deviations smaller than $\pm 0.3 \mathrm{~K}$. All side walls consist of double glazing polycarbonate plates with a $5 \mathrm{~mm}$ air gap and an additional $100 \mathrm{~mm}$ polystyrene insulation, in order to minimise heat losses through the side walls. The air inflow is supplied through a slot below the ceiling, while exhausting is provided by another slot above the floor. Both vents extend over the whole length of the cell. The working fluid is air at ambient pressure $(\operatorname{Pr}=0.71)$. The measurements were conducted using twelve PT-100 temperature sensors placed within the cell and another 86 PT-100 sensors 


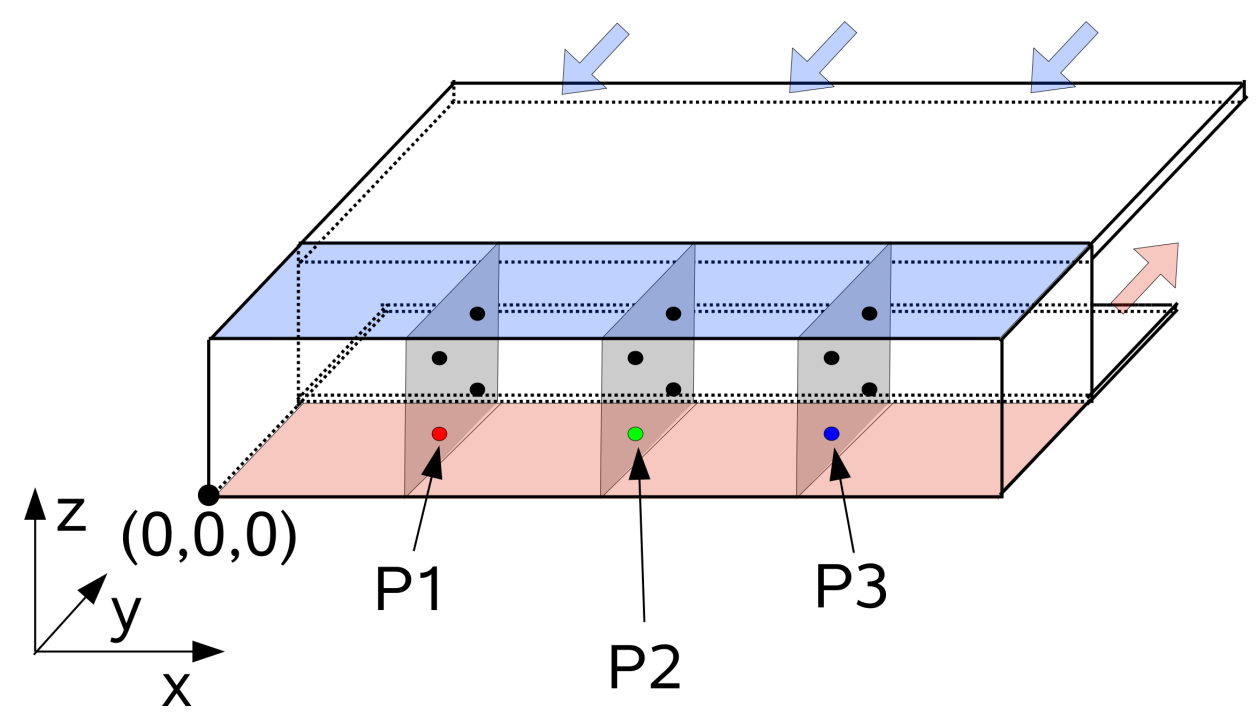

Figure 5.1: Sketch of the convection cell. The bottom of the cell is heatable (red) while the ceiling is kept at ambient temperature (blue). Inflow (blue arrows) and outflow (red arrows) channel are located at the same side and span the whole length of the cell. Black dots mark the positions of the PT-100 sensors within the cell.

for control reasons, e.g. in the cooling plate or the heating plate. Furthermore, the inflow velocity was monitored by controlling and measuring the volume flow rate during the experiment.

\subsection{Results}

We studied mixed convection in a range of $0.5<A r<6.9$ under variation of $\Delta T$ and $u$. It should be noted, that the heating system of the cell was running the whole time and that prior to each measurement the flow was allowed to stabilise for at least one hour after stable $R e$ and $R a$ were reached.

Figure 5.2 presents the result of a "stair-measurement" in which the inflow velocity was abruptly decreased in constant steps of $2.7 \mathrm{~cm} \cdot \mathrm{s}^{-1}$ every hour starting with $34.7 \mathrm{~cm} \cdot \mathrm{s}^{-1}$. This resulted in a stepwise change of Re in the range $1.1 \times 10^{4}>R e>0.7 \times 10^{4}$ while Ra was kept constant at $R a \cong 1.1 \times 10^{8}$. Consequently, $A r$ was increased in turn stepwise from $A r \cong 1.3$ to $A r \cong 3.1$ at the same time.

The resulting time series of the characteristic numbers are depicted in Fig. 5.2 (a), while Fig. 5.2 (b) shows the temperature time series of three selected sensors at $X / L=$ $0.25(P 1), 0.50(P 2)$ and $0.75(P 3)$ at the same $Y / W=0.25$ and $Z / H=0.20$. At the beginning of the measurement, i.e. for the highest $R e$, we found a stable configuration with warm air in the middle (P2) and cold air at one and three quarters of the cell (P1 and P3). This points to an arrangement of four convection rolls in the cell with warm air rising in the centre and at the sides as well as cold air falling in between. These convection rolls are superimposed on the forced mean flow, which leads to a non-uniform temperature distribution in a layer parallel to the bottom plate (not shown here for the sake of brevity). 


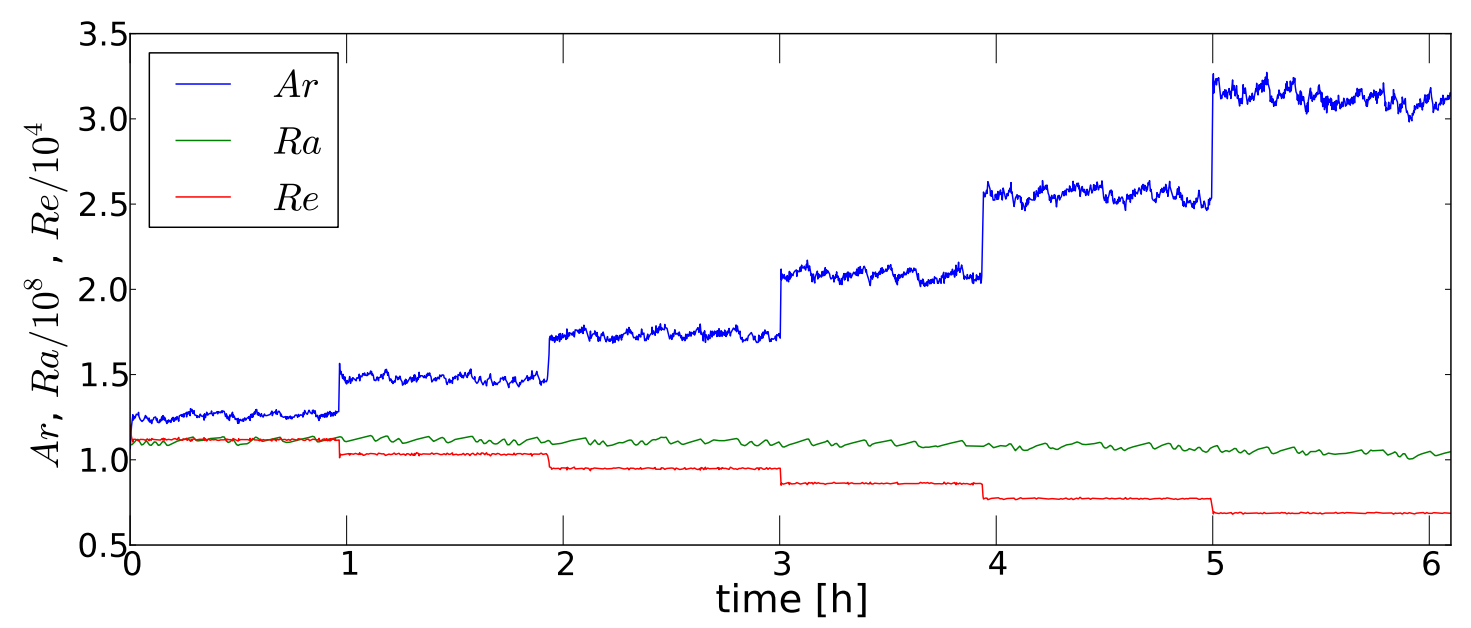

(a)

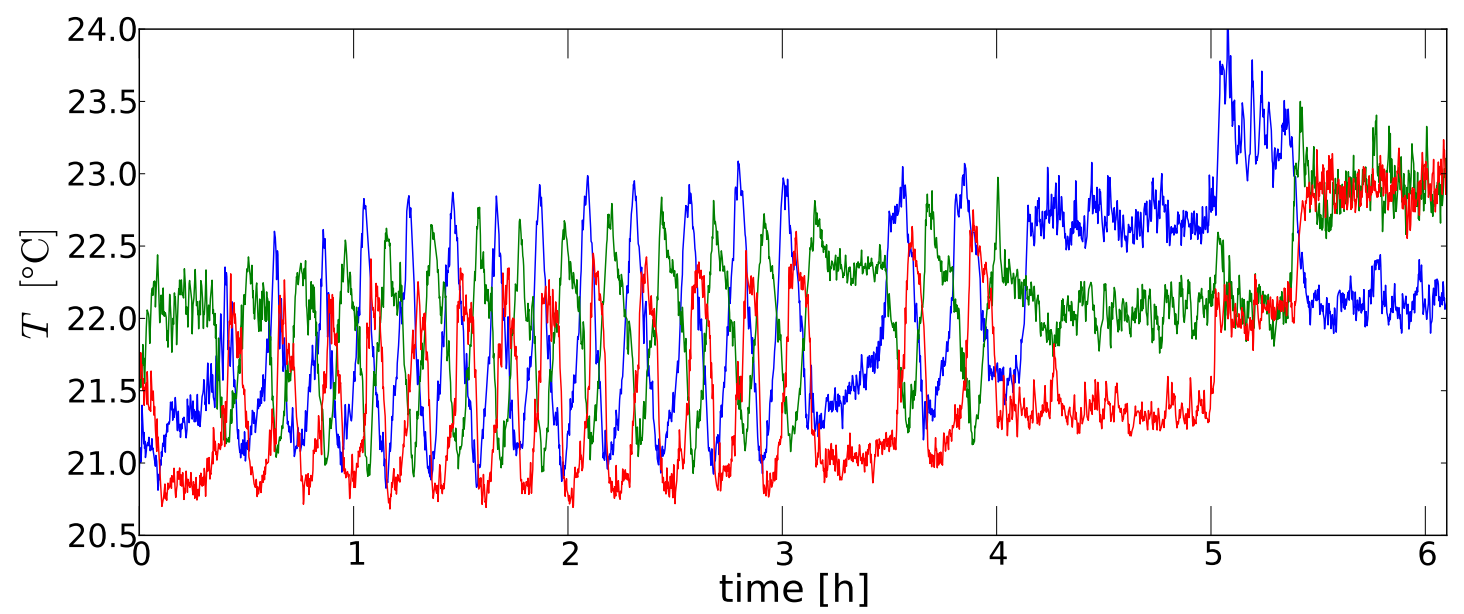

(b)

Figure 5.2: "Stair-measurement". (a) Time series of Re (red), Ra (green) and Ar (blue). (b) Temperature time series at P1 (red), P2 (green) and P3 (blue), see Fig. 5.1.

Warm air rises in the rear part of the cell whereas cold air descents close to the front wall. After approximately half an hour the temperatures start to oscillate with a frequency of $f \cong 1.2 \times 10^{-3} \mathrm{~Hz}$. Barely visible is that the frequency is slightly influenced by the change of $A r$ from $A r \cong 1.3$ to $A r \cong 1.5$ and $A r \cong 1.7$. An interesting point of this oscillation is that the centre (P2) region is the warmest for a few minutes, then suddenly cools down and meanwhile first at P3 and shortly afterwards at P1 the temperature increases strongly. The configuration with the cold air in the centre and warmest regions at one and three-quarters of the cell point to an inverted configuration to the firstly observed stable one.

Upon the further increase of $A r$ the oscillations suddenly disappear at $A r \cong 2.1$ until they start again after half an hour at the same $A r$ and finally stop completely upon the next increase to $A r \cong 2.6$. At this $A r$, the temperature field reflects warm air at P3, cold air at P1 and an intermediate temperature at P2. This temperature configuration points to the alignment of three large-scale convection rolls with rising warm air at $X \approx 0$ 
and $0.66 \times L$ and falling cold air at $X \approx 0.33$ and $1 \times L$. Combined smoke visualisations confirmed the existence of such a three-roll-configuration. After increasing $A r$ to the highest investigated value of $A r \cong 3.1$ another reorientation occurs, which we interpret as a short-circuit flow condition. The sudden increase of the mean cell temperature as well as a negative temperature gradient in $y$-direction indicate this. Inertia forces are now so small that the incoming air jet is not able to follow the ceiling, but descends directly towards the air outlet. Additionally, after approximately 30 minutes this state undergoes a spontaneous flow reversal as indicated by the temperature signals at P1, P2 and P3.

In this experiment the stepwise decrease of the inflow velocity, rather than a continuous one has been chosen mainly by experimental circumstances. Nevertheless, the changes in the topology result rather from a natural transition than the abrupt change of $R e$, because the same topologies were detected in long time measurements with constant characteristic numbers as well. Furthermore, the step size was just of the order of $10 \%$ and additionally, we performed a measurement with the same mean gradient, but a by a factor of five smaller step size. Again, periodic oscillations were found over a wide characteristic number range framed from some steady states.

Besides the "stair-measurement", other measurements were conducted under steady inflow and thermal boundary conditions. Figure 5.3 (a) and (b) show detailed views of temperature time series at two different $A r$ at constant $R a \cong 1.1 \times 10^{8}$. Again, three different dynamics are found. A stable temperature distribution (not shown here for the sake of brevity), a stable three roll configuration (3R) with a suddenly occurring change to four (4R), three counter rotating (3Rc), four counter rotating (4Rc) and back to the original three rolls (Fig. 5.3, a) as well as periodic oscillations (Fig. 5.3, b) are observed. An interesting fact is that the transition of the large-scale flow configuration seems to be the same for the spontaneously occurring (Fig. 5.3, a) case and the periodic (Fig. 5.3. b) one. The temperature decreases at P3 and simultaneously increases at P1. After approximately $200 \mathrm{~s}$ the temperature decreases at P1 to its initial value, while it rises at P2 and stays constantly low at P3. Approximately $200-400 \mathrm{~s}$ later all temperatures return to their initial values. We think, that the underlying motion of the large-scale circulation is a shift of the roll configuration in the longitudinal cell direction (x). Resulting in a change of the number and the rotation direction of the large-scale convection rolls from three rolls $(3 \mathrm{R})$ to four rolls (4R) to three counter rotating rolls (3Rc) to four counter rotation rolls (4Rc) and back to the initial three roll configuration (3R) (see Fig. 5.3, a and b).

Temperature time series were analysed using fast Fourier transform and a peak detection algorithm in order to determine the characteristic frequency. Figure 5.4 (a) shows the dependency of the found oscillation frequencies as a function of $R e$ and $A r$. The lines indicate constant $R a$. All marks depict a measured parameter combination. Crosses mark cases for which stable temperatures were found. Circles indicate cases with oscillations of the temperatures, thereby the colour of the circles is scaled by the value of the main frequency. So far, we did not find a simple dependency of the flow state on the characteristic numbers, which describes whether oscillations will occur, neither on $A r, R e, R a$ nor the Grashof 


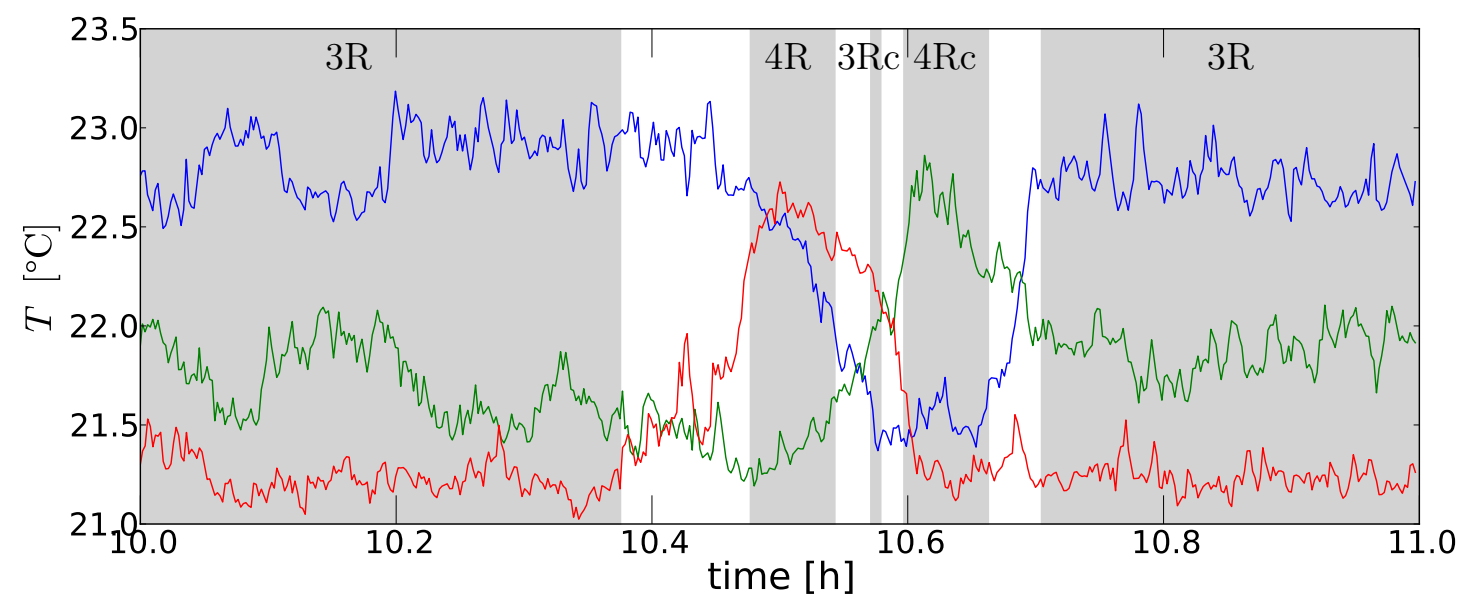

(a)

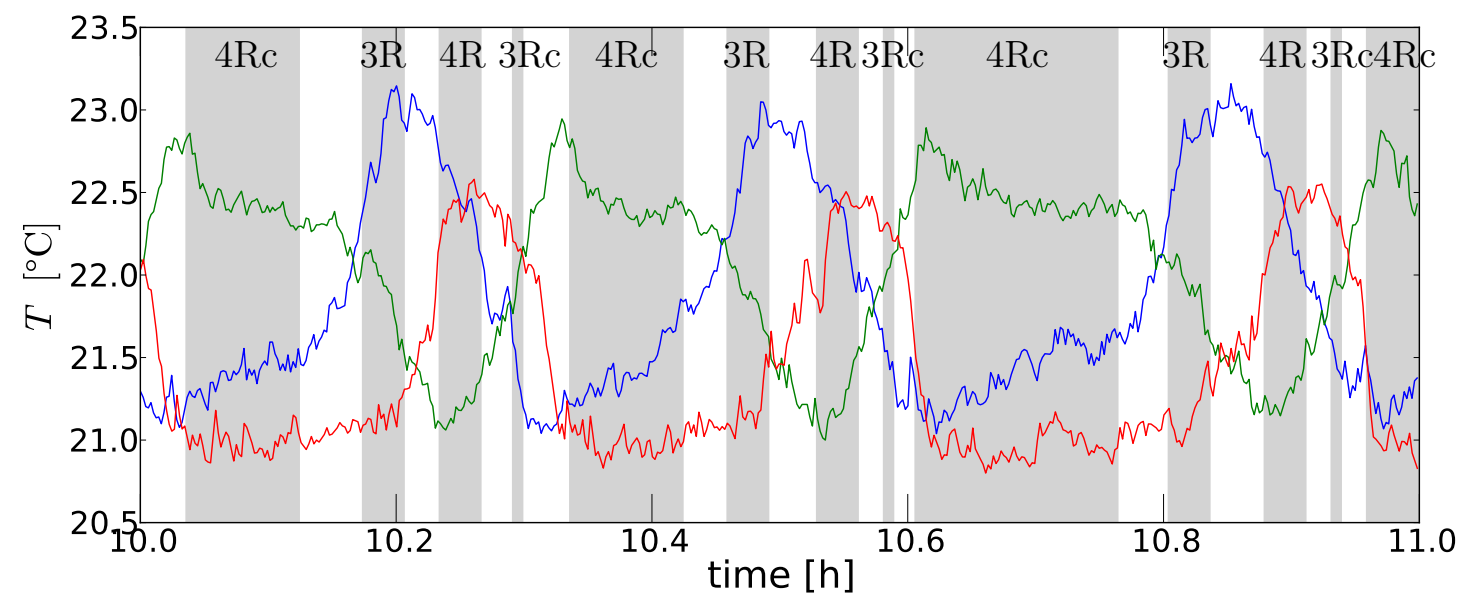

(b)

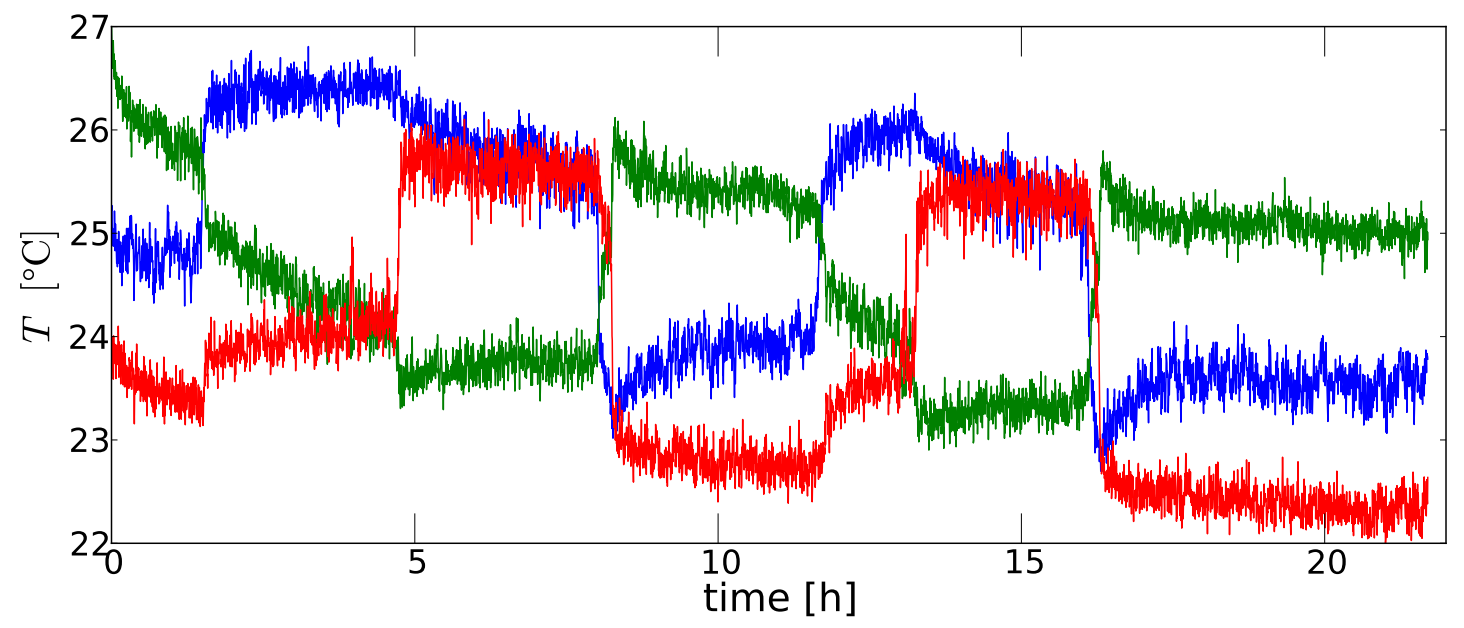

(c)

Figure 5.3: Temperature time series, P1 (red), P2 (green) and P3 (blue). One hour detail views (beginning at the measurement time of $10 \mathrm{~h}$ ) at $R a \cong 1.1 \times 10^{8}$ (a) $R e \cong 0.8 \times 10^{4}$ $(A r \cong 2.3)$ and $(\mathrm{b}) R e \cong 0.9 \times 10^{4}(A r \cong 1.9)$. (c) Full 22 hours temperature series at $R e \cong 1.4 \times 10^{4}, R a \cong 2.0 \times 10^{8}$ and $A r \cong 1.6$. 


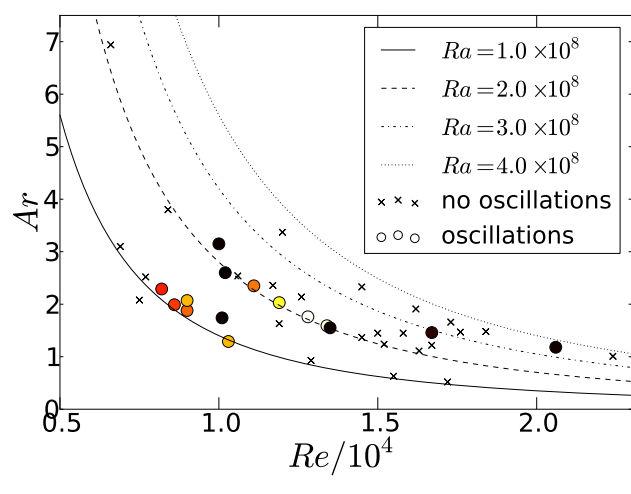

(a)

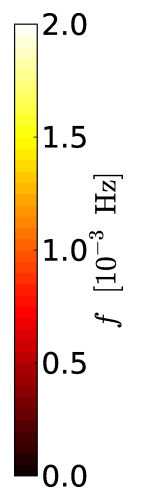

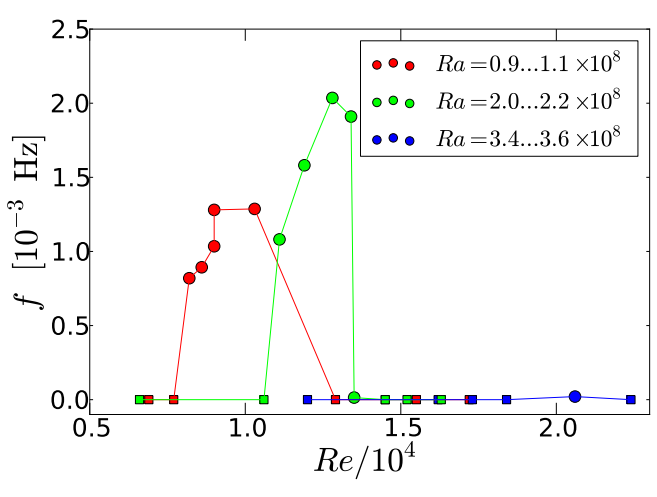

(b)

FiguRE 5.4: (a) Observed frequencies in the temperature time series in the $(A r, R e)$ parameter space, lines indicate constant $R a$ : Crosses mark cases with no periodic oscillations, while the colour of the circles is scaled with the value of the main frequency. (b) Main frequency as a function of $R e$ with $R a$ colour coded. Squares: no oscillations, circles: oscillations were detected.

number $G r$ (which is proportional to $R a$ ). Nevertheless, some statements restricting the degree of freedom of the occurrence of oscillations can be made. First, the smaller Re and the smaller $R a$ the higher is the possibility for the occurrence of oscillations. Second, for a given (and not too high) $R a$, oscillations occur only in an intermediate regime of $A r(R e)$, neither at too high nor at too low values. Third, with increasing $R a$ oscillations occur at higher Re $(A r)$ and have higher frequencies (see Fig. 5.4 , b).

Furthermore, we found some very low frequencies, e.g. $f \cong 2.0 \times 10^{-5} \mathrm{~Hz}$ at $R a \cong 2.0 \times 10^{8}$, $R e \cong 1.4 \times 10^{4}$ and $A r \cong 1.6$, meaning that there were just two complete oscillations during a measurement time of almost $22 \mathrm{~h}$, see Fig. 5.3 (c). As opposed to the dynamics observed in Fig. 5.3 (a) and (b) complete reversals of the rotation direction as well as a change of the number of convection rolls occurred for this parameter configuration.

Sergent and Le Quéré [106] found in Rayleigh-Bénard convection the existence of two, three or four convection rolls as a function of time as well as reversals of their rotation directions, but no fast oscillations as depicted in Fig. 5.3 (b). We ascribe the occurrence of the oscillations to the interaction between the superimposed shear forces of the forced convective flow to the buoyancy driven flow.

Ongoing measurements with more sensors revealed for $R a \cong 2 \times 10^{8}(1.3<A r<2.3$ and $\left.1.1 \times 10^{4}<R e<1.5 \times 10^{4}\right)$ that the oscillations are a continuous sequence with four, three, four counter rotating and three counter rotating convection rolls within the cell.

\subsection{CONCLUSion}

Temperature oscillations, which we observed during experiments in turbulent mixed convection in a rectangular enclosure, were analysed. These temperature fluctuations are ascribed to oscillations and spontaneous reorientations of the large-scale circulations in 
the flow. Stable temperature configurations, periodic oscillations as well as spontaneous reversals and reorientations were detected. So far, we could not find a simple dependency on the characteristic numbers $(A r, R e$ and $R a)$ which describes whether oscillations will occur. But we can state the following: The smaller $R e$ and the smaller $R a$ the higher is the possibility for the occurrence of oscillations. Furthermore, with increasing $R a$, oscillations occur at higher $R e(A r)$ and have higher frequencies. Due to the fact that the occurrence of the oscillations and the spontaneous reorientations of the large-scale flow depend on both $R e$ and $R a$, we ascribe them to the interaction between the superimposed shear forces of the forced convective flow to the buoyancy driven flow.

Measurements with more sensors as well as combined temperature and velocity measurements are currently performed and are expected to shed more light on the observed processes. 


\title{
6 Oscillations of THE LARGE-SCALE Circulations 98
}

Citation and credit: Reprinted with permission from Experiments in Fluids, volume 54, article 1517, 2013, DOI:10.1007/s00348-013-1517-3, Copyright 2013, Springer-Verlag Berlin Heidelberg

Reference: 98

Title: "Oscillations of the Large-Scale Circulations in Turbulent Mixed Convection in a Closed Rectangular Cavity"

Authors: Daniel Schmeling, Johannes Bosbach and Claus Wagner

Contributions: I performed all measurements, analysed them and made all figures. I wrote the first draft, whose structure has been preserved.

\begin{abstract}
Fluid temperature time series are recorded in turbulent mixed convection at specific locations inside a cuboidal convection cell. They reveal instabilities of the large-scale flow structures, which organise the heat transport in an intermediate range of Archimedes numbers, where buoyancy and inertia forces are of similar strength. The instabilities lead to periodic or spontaneous transitions between three and four convection rolls. Further, for either high Rayleigh or Reynolds numbers, for which the flow is either governed by buoyancy or by inertia forces, respectively, stable large-scale circulations (LSCs) develop. In the intermediate $R a-R e$ number regime, we ascribe the complex dynamics, visible as oscillation in the temperature time series, to the interaction of the pressure-driven wall jet at the ceiling with the buoyancy-driven LSCs. The maximal main oscillation frequency is about one order of magnitude smaller than the turnover frequencies of either the wall jet-induced circulation rolls or thermally induced LSCs. It is further shown that the periodic reconfigurations of the LSCs can be controlled by adjusting the inflow velocity, that is, the Reynolds number, to generate stable LSCs.
\end{abstract}




\subsection{INTRODUCTION}

Mixed convection (MC) denoting the combination of the pressure-driven forced convection (FC) and buoyancy-driven thermal convection (TC) occurs abundantly in nature and in many technical applications. In these flows, the Archimedes number $A r=R a \times R e^{-2} \times \operatorname{Pr}^{-1}$ expresses the ratio between buoyancy and inertia forces with $R a, R e$ and $\operatorname{Pr}$ denoting the Rayleigh, Reynolds and Prandtl number, respectively.

Of great economic and ecologic relevance are dynamics and size of the LSCs in MC which organise the heat transport for example in heat exchangers, rooms or more specifically in cabins of aircraft, trains and other vehicles. In the latter passengers and electrical devices induce TC and the air flow of the air-conditioning system superimposes FC. Studies in this field have been conducted in generic enclosures [17], full-scale aircraft cabin mock-ups [64] and flight tests [13]. The analysis of the dynamics of the LSCs in realistic cabin environments is hampered by the geometric complexity. It is well known that the heat transport in these environments is closely related to the large- and small-scale turbulent flow structure. This interaction is also important for development of energy efficient ventilation systems [13, 15].

Since ventilation in aircraft cabins is to a very high degree driven by TC [13, 15, the dynamics and spatial distribution of the LSCs in TC have recently gained importance also from a technological point of view. In this respect, fundamental studies of pure TC, that is, Rayleigh-Bénard convection (RBC), reveal that the arrangement of the LSCs governs the local heat transport 107, 132, 138, 149,. The development and orientation of LSCs generated in TC in a cuboidal Rayleigh-Bénard cell with aspect ratio five were investigated by large eddy simulations (LES) 81, 106 and direct numerical simulations (DNS) 60. The analysis of the LES data revealed two, three and four roll configurations as well as spontaneous transitions.

Furthermore, Ishihara et al. [58 determined the spatial temperature distribution in their TC experiments in a rectangular enclosure. Depending on $R a$ and the depth of their cuboidal sample, they obtained the following three different flow states: A steady quasi-two-dimensional flow, a steady three-dimensional flow and an unsteady flow state.

Dynamic effects like rotations, cessations, spontaneous reorientations and even torsional oscillations as well as a "sloshing mode" of the LSCs are well known from turbulent RBC in cylindrical geometries $20,24,25,73,150$. Sreenivasan et al. 118 proposed that the reversals are triggered by an imbalance of buoyant and frictional forces, with inertia playing a secondary role. Furthermore, they suggested that the reversals are a result of various boundary layer instabilities like jets and plumes. Fontenele Araujo et al. [39] analysed the wind reversals in turbulent RBC and developed a numerical model to describe the regimes of uniform circulations as well as chaotic and periodic reversals in the $\operatorname{Ra} \times \operatorname{Pr}$ phase space.

Several physical pictures and models based on different simplifying assumptions are developed for these numerous dynamic processes in pure RBC [21, 22, 39, 89, 118]. These pictures help to understand the process of a reversal, whereas the different models reproduce the most important dynamical processes. For example, Brown and Ahlers 21,22 based 
their model on two ordinary differential equations, one for the strength and one for the azimuthal orientation of the LSC. The dynamics of the model is a result of the addition of statistical forces. These forces can be interpreted as the action of the turbulent fluctuations that exist throughout the system.

The results of the above discussed studies which address the formation and the dynamics of LSC in pure TC are transferable to MC for very high Ar only. Further, little is known about the organisation and stability of the LSCs in MC. Nevertheless, it seemed worthwhile to review the TC studies, to see which ideas or concepts might be adoptable to MC.

A series of studies, which address a few of the above mentioned issues, has been performed recently in a rather generic configuration by means of DNS and experiments [5, 62, 109]. However, the investigations have been performed either under isothermal conditions or on time scales too short to study the low frequent behaviour of the large-scale structures. Also previous investigations of the flow in the cuboidal sample addressed below, focus on the structure formation, heat transport and low-frequency oscillations $66,67,104,105: 140]$. Their temperature measurements revealed oscillations in the outflowing air, and the arrangement of LSCs as well as oscillations of time development coefficients of Proper Orthogonal Decomposition (POD) of the velocity fields were analysed. Furthermore, plume length and plume spacing were studied in pure TC in our cuboidal cavity [83]. Though, their work did not focus on the dynamics of the LSCs in detail. To fill this gap, the following questions will be addressed below:

1. How do the LSCs arrange in a cuboidal convection cell of aspect ratio five as a function of the characteristic numbers?

2. How can the stability as well as the dynamics of the LSCs be characterised and how does the dynamics scale with the characteristic numbers?

Finally, we give some possible explanations concerning the question how the stability and the dynamic process of the LSCs can be modelled with reference to the characteristic numbers.

The paper is outlined as follows: Section 6.2 describes the experimental setup. In section 6.3 , the experimental results of the temperature measurements are presented and discussed. The compulsive conclusion is given in the last section.

\subsection{EXPERIMENTAL SETUP AND PROCEDURE}

$\mathrm{MC}$ is studied in a rectangular convection cell of length $L=2,500 \mathrm{~mm}$ with the aspect ratios $\Gamma_{\mathrm{xz}}=\frac{L}{H}=5$ and $\Gamma_{\mathrm{yz}}=\frac{W}{H}=1$, where $H$ denotes the height and $W$ the width of the container. A sketch of the cell is shown in Figure 6.1. In order to accomplish nearly isothermal boundary conditions, the heating device at the bottom consists of five separate sub-plates, each of them being actively heated via a temperature- controlled water circuit. The ceiling is passively cooled close to ambient temperature by an air-air heat exchanger. The side panels are a double-wall system of polycarbonate plates, which guarantee good 
optical access as well as minimal heat loss. The heat losses are further reduced with an additional layer of $100 \mathrm{~mm}$ polystyrene insulation installed for the temperature measurements, for which no optical access was necessary. By operating the heating plates, a vertical temperature gradient is generated to induce TC. Furthermore, the cell is equipped with an air inlet at the top and an air outlet at the bottom. The heights of the inlet and the outlet channels amount to $25 \mathrm{~mm}$ and $15 \mathrm{~mm}$, respectively. Both are located on the same side wall and extend over the full length of the container. This configuration allows to generate a flow between the in- and outlet driven by the applied pressure gradient. A more detailed description of the convection cell can be found in 105.

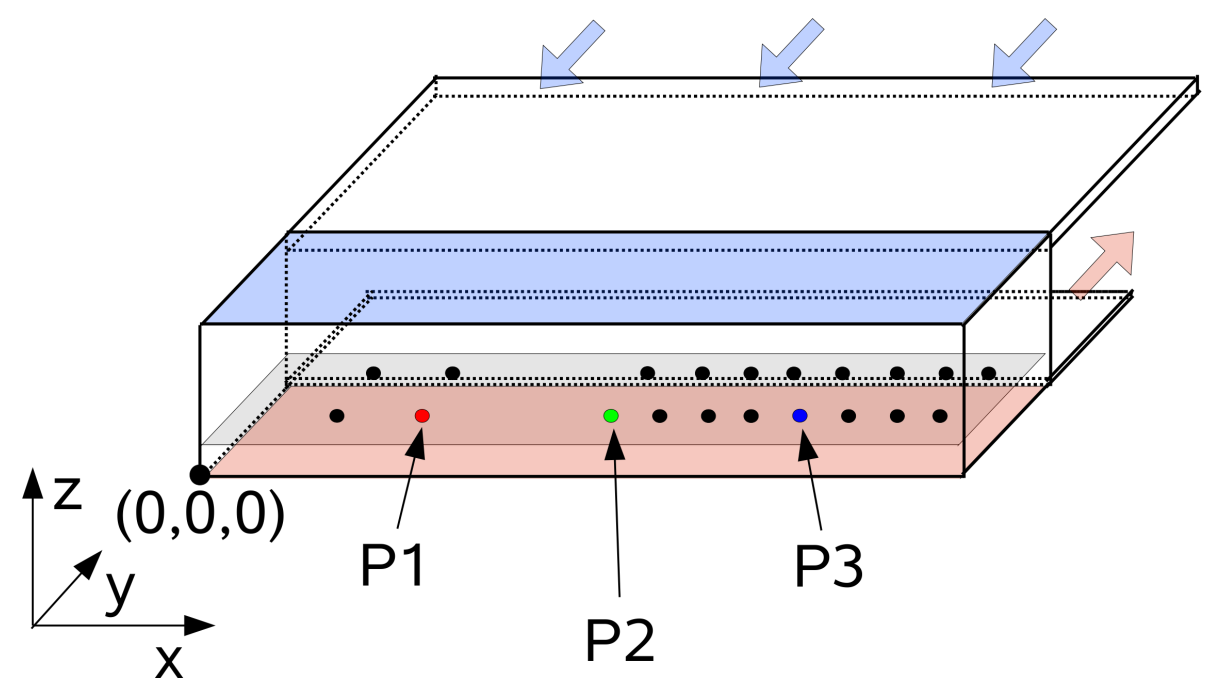

Figure 6.1: Sketch of the convection cell. The bottom plate of the cell is heated (red), while the ceiling is kept at ambient temperature (blue). The in- and outflow channels are located at the same side wall and span the whole length of the cell. Dots mark the positions of the Pt100 sensors in the layer $Z / H=0.2$ within the cell. More Pt100 sensors are placed in the layer $Z / H=0.8$, but are not shown for the sake of clarity.

Temperature measurements were performed with 35 Pt100 (1/3 DIN B) temperature sensors placed within the cell and 86 more Pt100 sensors installed in the cooling and heating plates as well as in the in- and outlet. The sample rate amounted to $f \approx 1 / 9 \mathrm{~Hz}$.

The temperature distributions in the cooling and the heating plates were measured with 25 sensors each. They revealed spatial temperature deviations of $\Delta T_{\mathrm{c} \text {,std }}=0.6 \%$ and $\Delta T_{\mathrm{h}, \mathrm{std}}=1.1 \%$. Here, the suffix $\mathrm{h}$ denotes the heating and $\mathrm{c}$ the cooling device, while the index std labels the standard deviation. All temperature deviations are normalised with the temperature difference of the heating and the cooling plates, averaged in space and time over all measurement series, that is, $\Delta T=17.18 \pm 0.34 \mathrm{~K}$. The $2 \mathrm{D}$ spatial temperature distributions of the cooling and the heating plates are depicted in Figure 6.2 (a) and (b), respectively. They reveal the time-averaged temperatures of the 25 sensors, whose positions are marked as black dots. It is observed that the respective maximal lateral temperature differences on the cooling and the heating plates are smaller than $\pm 0.35 \mathrm{~K}$.

The temperature of the incoming air is measured with three sensors. The obtained 


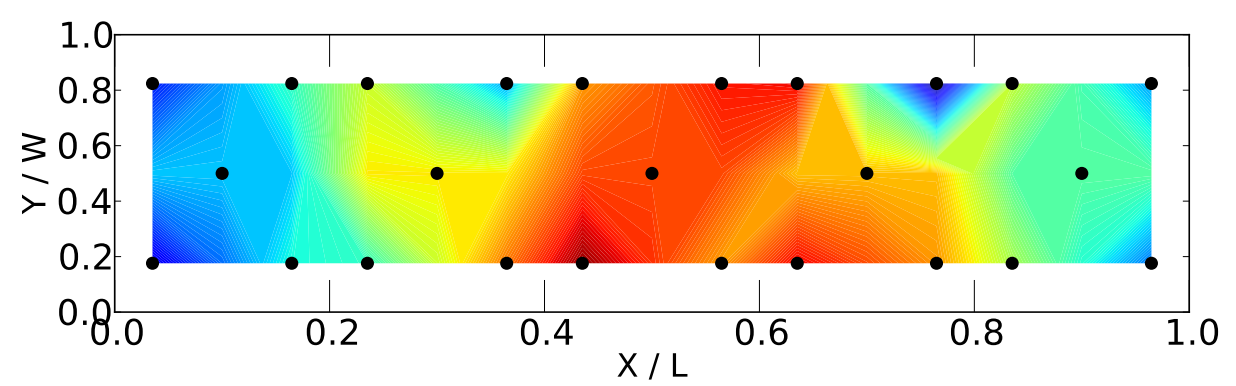

(a)

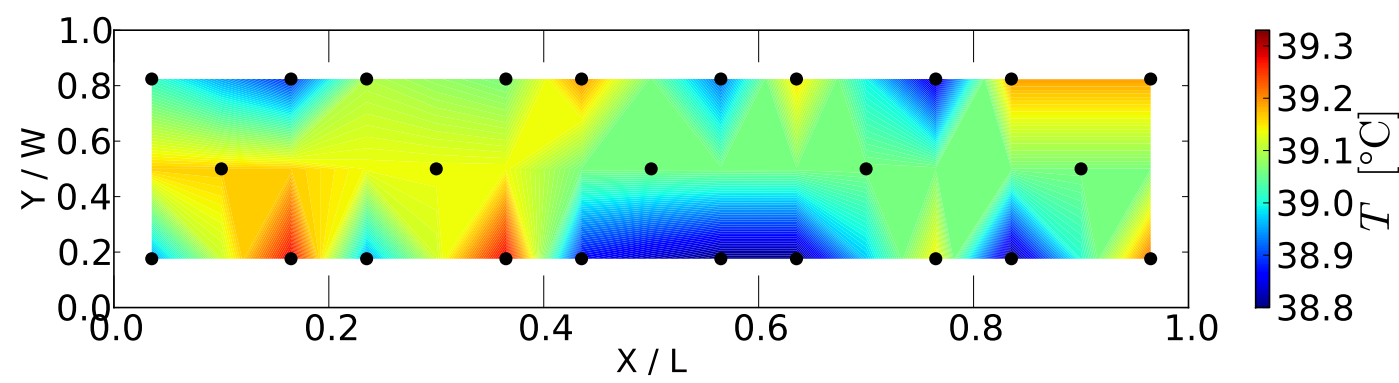

(b)

Figure 6.2: $2 \mathrm{D}$ time-averaged temperature distributions in the cooling plate (a) and the heating plate (b) at mean temperatures of $\left\langle T_{c}\right\rangle=21.6^{\circ} \mathrm{C}$ and $\left\langle T_{h}\right\rangle=39.0^{\circ} \mathrm{C}$. Temperatures are measured at the positions which are marked as black dots.

standard deviation with respect to the mean temperature values averaged in space and time is $\Delta T_{\mathrm{in}, \mathrm{std}}=0.7 \%$. To determine the temperature deviations of the leaving air, nine sensors are installed equidistantly over the full length at the end of the outlet channel. More Pt100 temperature sensors are used within the cell to analyse of the fluid temperatures. These 35 sensors are mainly located at a height of $Z / H=0.20$ to provide an adequate spatial resolution within this layer. Another six sensors are installed at $Z / H=0.80$ and another three at various $Z$ positions. Table 6.1 summarises the positions of all sensors within the convection cell.

In the following, we will mainly concentrate on the signals of three temperature probes located at $Z / H=0.2, Y / W=0.25$ as well as $P 1: X / L=0.25, P 2: X / L=0.50$ and $P 3: X / L=0.75$ (P1 red, P2 green and P3 blue), see also Table 6.2 and Figure 6.1 .

To determine the Prandtl number $\operatorname{Pr}=\frac{\nu}{\kappa}$, Reynolds number $R e=\frac{U \cdot H}{\nu}$, Rayleigh number $R a=\frac{g \cdot \beta \cdot \Delta T \cdot H^{3}}{\nu \cdot \kappa}$ and Archimedes number $A r=\frac{R a}{P r \cdot R e^{2}}$, the fluid's kinematic viscosity $(\nu)$, thermal diffusivity $(\kappa)$ and isobaric thermal expansion coefficient $(\beta)$ are assumed to be a function of temperature only. Assuming a constant static pressure of $p=1$ bar is an acceptable assumption, since the ambient pressure varied between 950 and 1,050 hPa during the measurement campaign, which results in changes for properties $\nu, \kappa$ and $\beta$ of less than $3 \%$. For completeness, it should be mentioned that $g$ stands for the gravitational acceleration and $H, U$ as well as $\Delta T$ for the height of the cell, the mean inflow velocity and the temperature difference between bottom and ceiling of the cell, respectively. 
TABLE 6.1: Temperature sensor positions within the convection cell for the analysis of the fluid temperatures.

\begin{tabular}{lllllllll}
\hline$X / L$ & $Y / W$ & $Z / H$ & $X / L$ & $Y / W$ & $Z / H$ & $X / L$ & $Y / W$ & $Z / H$ \\
\hline $1 / 16$ & $1 / 4$ & $1 / 5$ & $1 / 8$ & $1 / 4$ & $1 / 5$ & $1 / 8$ & $3 / 4$ & $1 / 5$ \\
$3 / 16$ & $1 / 4$ & $1 / 5$ & $1 / 4$ & $1 / 4$ & $1 / 5$ & $1 / 4$ & $3 / 4$ & $1 / 5$ \\
$1 / 2$ & $1 / 4$ & $1 / 5$ & $1 / 2$ & $3 / 4$ & $1 / 5$ & $9 / 16$ & $1 / 4$ & $1 / 5$ \\
$9 / 16$ & $3 / 4$ & $1 / 5$ & $19 / 32$ & $3 / 8$ & $1 / 5$ & $19 / 32$ & $5 / 8$ & $1 / 5$ \\
$5 / 8$ & $1 / 4$ & $1 / 5$ & $5 / 8$ & $3 / 4$ & $1 / 5$ & $21 / 32$ & $3 / 8$ & $1 / 5$ \\
$21 / 32$ & $5 / 8$ & $1 / 5$ & $11 / 16$ & $1 / 4$ & $1 / 5$ & $11 / 16$ & $3 / 4$ & $1 / 5$ \\
$3 / 4$ & $1 / 4$ & $1 / 5$ & $3 / 4$ & $3 / 4$ & $1 / 5$ & $13 / 16$ & $1 / 4$ & $1 / 5$ \\
$13 / 16$ & $3 / 4$ & $1 / 5$ & $7 / 8$ & $1 / 4$ & $1 / 5$ & $7 / 8$ & $3 / 4$ & $1 / 5$ \\
$15 / 16$ & $1 / 4$ & $1 / 5$ & $15 / 16$ & $3 / 4$ & $1 / 5$ & & & \\
\hline $1 / 4$ & $1 / 4$ & $4 / 5$ & $1 / 4$ & $3 / 4$ & $4 / 5$ & $1 / 2$ & $1 / 4$ & $4 / 5$ \\
$1 / 2$ & $3 / 4$ & $4 / 5$ & $3 / 4$ & $1 / 4$ & $4 / 5$ & $3 / 4$ & $3 / 4$ & $4 / 5$ \\
\hline $5 / 8$ & $1 / 2$ & $1 / 50$ & $5 / 8$ & $1 / 2$ & $1 / 2$ & $5 / 8$ & $1 / 2$ & $49 / 50$ \\
\hline
\end{tabular}

TABLE 6.2: Three selected temperature sensor positions, see also Figure 6.1

\begin{tabular}{lllll}
\hline Name & Colour & $X / L$ & $Y / W$ & $Z / H$ \\
\hline P1 & red & 0.25 & 0.25 & 0.20 \\
P2 & green & 0.50 & 0.25 & 0.20 \\
P3 & blue & 0.75 & 0.25 & 0.20 \\
\hline
\end{tabular}

The fluid properties are calculated using the mean fluid temperature $<T_{\text {fluid }}>$, which is determined by averaging the recorded temperatures of twelve symmetrically arranged sensors in the fluid. Second-order polynomials 6.1 based on data of the "VDI-Wärmeatlas" 134 are used to take into account the effect of the mean temperature on the fluid properties:

$$
\{\nu, \kappa, \beta\}=a_{\{\nu, \kappa, \beta\}} \cdot<T_{\text {fluid }}>^{2}+b_{\{\nu, \kappa, \beta\}} \cdot<T_{\text {fluid }}>+c_{\{\nu, \kappa, \beta\}} \cdot
$$

For each fit, eleven data points are used in the range $0^{\circ} \mathrm{C} \leq<T_{\text {fluid }}>\leq 100^{\circ} \mathrm{C}$ in steps of $\Delta<T_{\text {fluid }}>=10 \mathrm{~K}$.

During all measurements, the temperatures of the cooling and heating plates and the inflow velocity are monitored. Thus, the time variations of characteristic numbers are considered.

To give an example, the time series of the characteristic numbers obtained for $A r=2.7$ (shown in Figure 6.3) demonstrates that, after a settling time of approx. $4 \mathrm{~h}$, all characteristic numbers fluctuate around a constant mean value.

Smoke visualisations are performed using two continuous wave lasers with a wavelength 


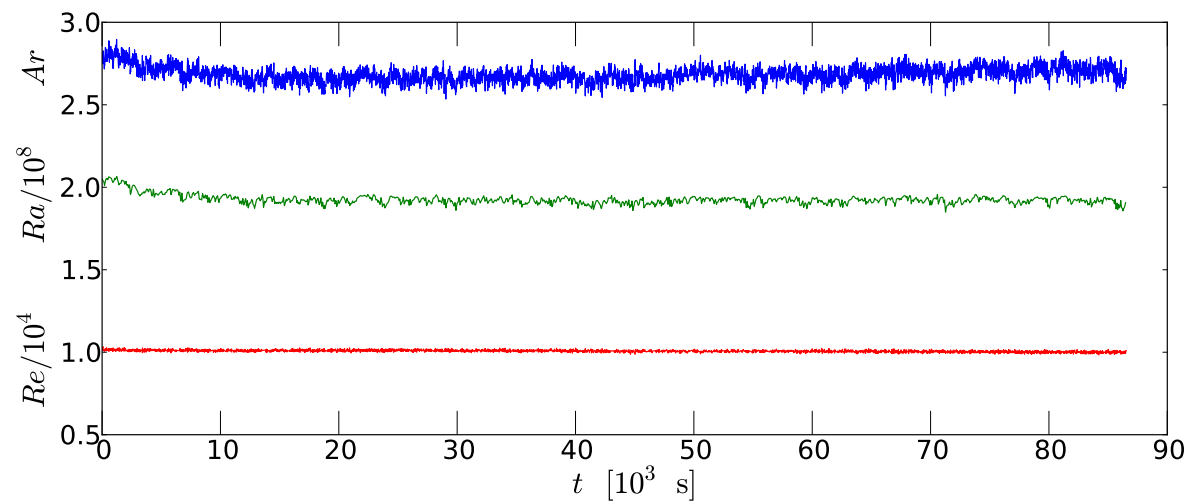

FiguRE 6.3: Representative time series of the characteristic numbers: Ar (blue), Ra (green) and Re (red).

$\lambda=532 \mathrm{~nm}$ and a nominal output power $P=200 \mathrm{~mW}$. Both lasers are equipped with light sheet optics consisting of a convex and a concave spherical lens as well as a cylindrical one. The light sheets are coupled into the convection cell through both end faces in order to provide a homogeneous illumination over the whole cell length. The smoke is injected into the suction opening of the fan, which is used to apply the external pressure gradient between the in- and outlet of the cell.

Images are recorded using a PCO.4000 camera equipped with 21-mm Zeiss lenses in the single shutter mode at approximately 6 frames per second. To achieve a better contrast in the recorded images, a local normalisation of the intensity values is applied. This technique is comparable to the high dynamic range (HDR) technique, well known from image manipulation software (see e.g. 45]), and results in images with fewer bright or dark saturated regions.

\subsection{Results AND DisCUSSION}

This section is outlined as follows: first, we will present temperature time series during a variation of $R e$ and $A r$, which show static and dynamic LSCs. Second, we will discuss smoke visualisations together with corresponding temperature profiles. Third, we will analyse three systematically different temperature time series recorded at sensor positions which give an impression of the considered periodic oscillations. Based on these temperature time series, we will draw conclusions regarding the structure of the LSCs within the cell. Finally, we will perform a frequency analysis and try to picture the dynamics of the LSCs with a mechanical model.

To make sure that the flow reached its equilibrium for each parameter combination, the following measurements are conducted in phases for which the characteristic numbers are maintained, that is, after long enough settling times, if not otherwise stated (see "staircase measurement"). In specific, we allowed at least a few hours of the total measurement time of more than $18 \mathrm{~h}$ for the flow to settle before we started averaging or comparing the different 
states. For many parameter combinations, we repeated the measurement at different days and found that the results are reproducible.

Table 6.3 summarises the investigated parameter range of $A r$ and $R e$ together with the obtained mean temperatures for a constant $R a=2.0 \times 10^{8}$, exemplarily. The mean fluid temperature increases with $A r$ because the Reynolds number decreases. The latter reflects that less cold air enters the cell, while the temperature difference $\Delta T$ remains nearly constant. More measurements were conducted in the range of $0.8 \times 10^{8} \leq R a \leq 3.6 \times 10^{8}$.

TABLE 6.3: Investigated parameter range at constant $R a \approx 2.0 \times 10^{8}$. The mean fluid temperature $<T_{\text {fluid }}>$ was calculated as mean value of twelve symmetrically arranged sensors within the cell (in the layers $X / L \in\{0.25,0.50,0.75\}, Y / W \in\{0.25,0.75\}$ and $Z / H \in\{0.20,0.80\})$.

\begin{tabular}{llllll}
\hline$U_{\text {in }}[\mathrm{m} / \mathrm{s}]$ & $\Delta T[\mathrm{~K}]$ & $\operatorname{Re}\left[\times 10^{4}\right]$ & $A r[1]$ & $<T_{\text {fluid }}>\left[{ }^{\circ} \mathrm{C}\right]$ & $<T_{\text {in }}>\left[{ }^{\circ} \mathrm{C}\right]$ \\
\hline 0.51 & 17.4 & 1.63 & 1.11 & 23.8 & 18.7 \\
0.48 & 17.1 & 1.52 & 1.24 & 24.4 & 19.0 \\
0.45 & 17.1 & 1.45 & 1.37 & 24.4 & 18.6 \\
0.43 & 17.1 & 1.34 & 1.59 & 24.6 & 18.7 \\
0.43 & 17.2 & 1.35 & 1.57 & 24.5 & 18.7 \\
0.40 & 17.3 & 1.28 & 1.76 & 24.4 & 18.4 \\
0.37 & 17.3 & 1.19 & 2.03 & 24.6 & 18.3 \\
0.35 & 17.2 & 1.10 & 2.35 & 24.8 & 18.2 \\
0.33 & 17.1 & 1.06 & 2.53 & 25.0 & 18.1 \\
0.32 & 16.6 & 1.01 & 2.68 & 25.5 & 18.1 \\
\hline
\end{tabular}

\subsubsection{Multiple LSCs DURing VARIATion OF $R e$ AND $A r$}

To demonstrate the transition paths between the possible multiple static and dynamic LSCs, we conducted a "staircase measurement". Thereby, the inflow velocity was lowered every hour by a value of $\Delta U \approx 2.7 \times 10^{-2} \mathrm{~ms}^{-1}$ while maintaining $\Delta T$. This results in a time development of the characteristic numbers as presented in Figure 6.4 (a): while $R a$ is kept constant, $R e$ decreases and thus $A r$ increases stepwise.

It should be noted explicitly that we do not expect to see the "final" states of the flow for each parameter combination, because a settling time of 1 hour is too short for the flow to relax. We rather want to demonstrate the transition between the different LSCs as a result of the variation of $R e$ and $A r$. Measurements at constant parameter combinations and much longer time periods and, thus, settling times are discussed hereafter.

The temperature time series at P1, P2 and P3 obtained during the "staircase measurement" are shown in Figure 6.4 (b). They reveal many different phases with steady LSCs, for example, for $0 \lesssim t \lesssim 1.5 \times 10^{3} \mathrm{~s}$ and $15 \times 10^{3} \mathrm{~s} \lesssim t \lesssim 18 \times 10^{3} \mathrm{~s}$, spontaneous occurring reconfigurations of the LSCs, for example, at $t \approx 1.5 \times 10^{3} \mathrm{~s}$ and $t \approx 19.5 \times 10^{3} \mathrm{~s}$ as well 


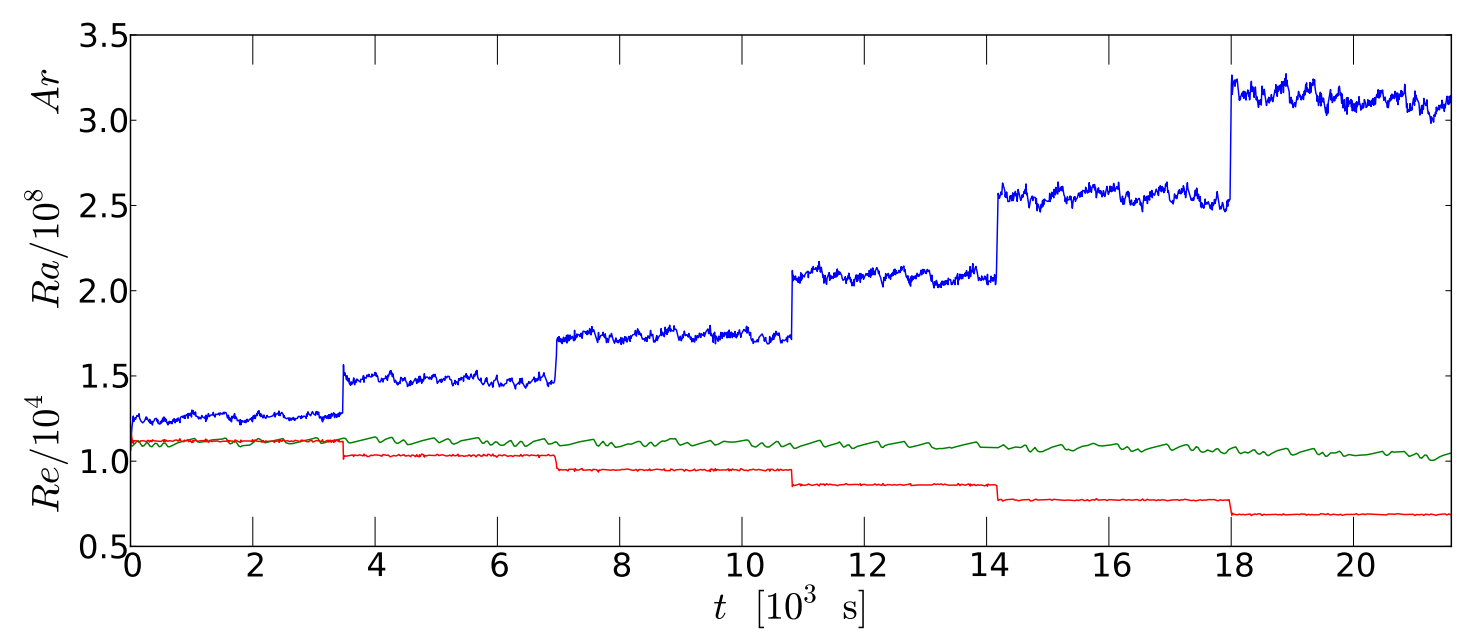

(a)

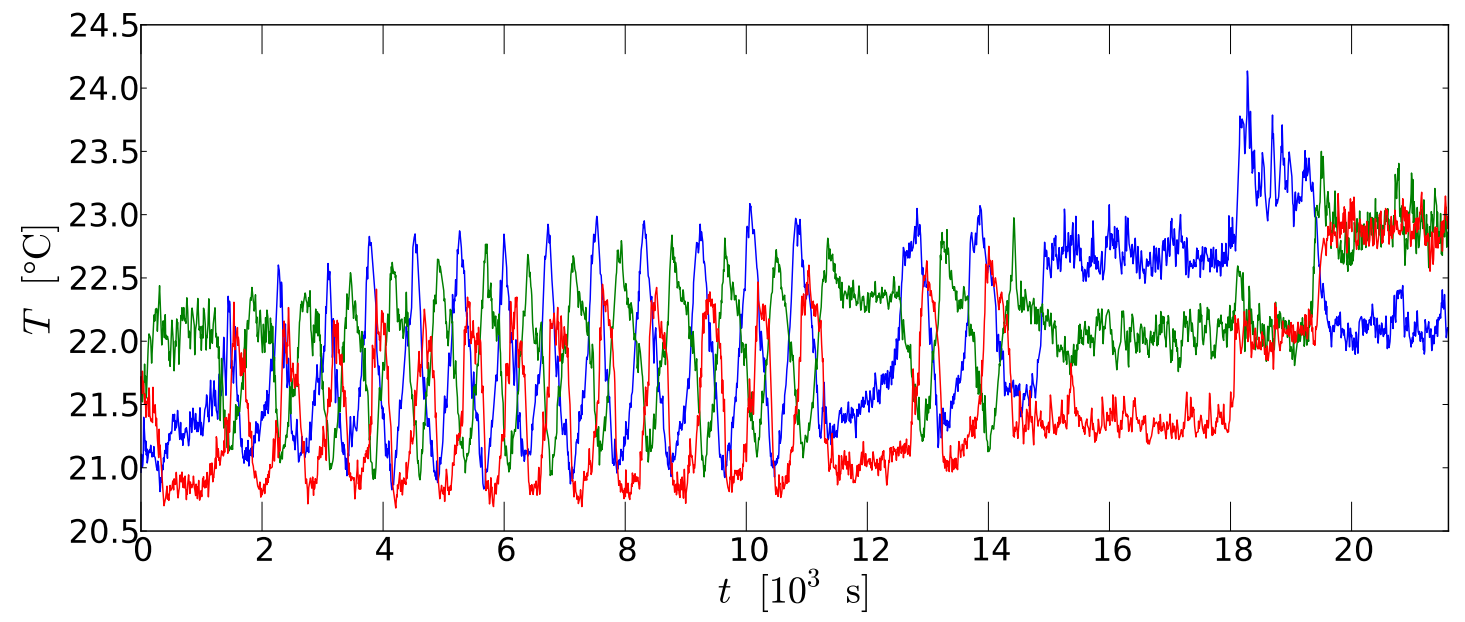

(b)

FiguRE 6.4: (a) Time series of the characteristic numbers: Ar (blue), Ra (green) and Re (red). (b) Corresponding temperature time series at three selected sensor positions: P1 (red), P2 (green) and P3 (blue), see Table 6.2 and Figure 6.1

as LSCs with periodic oscillations for $3 \times 10^{3} \mathrm{~s} \lesssim t \lesssim 11 \times 10^{3} \mathrm{~s}$. The existence of these multiple static and dynamic cases is the motivation for the following discussions.

\subsubsection{TEMPERATURE PROFILES AND SMOKE VISUALISATIONS}

In this subsection, we want to try to answer the question how the temperature signals correlate with the LSC configurations. We used smoke to visualise the LSCs in time intervals of nearly constant temperature signals, during which the additional polystyrene insulation of the convection cell was partly removed. Consequently, the thermal boundary conditions slightly deviate from those measurements conducted with the complete insulation for the same characteristic numbers. The aim of these visualisations is to link the events in the recorded temperature time series to changes in the structure of the LSCs. We discuss two 
examples of stable temperature distributions, that is, a four roll configuration with cold air descending in the centre (see Figure 6.5) and a three roll configuration with cold air descending at the right side wall (see Figure 6.6). Both figures include (a) the temperature time series at P1, P2 and P3, (b) snapshots of the corresponding smoke visualisations and (c) sketches of the LSCs.

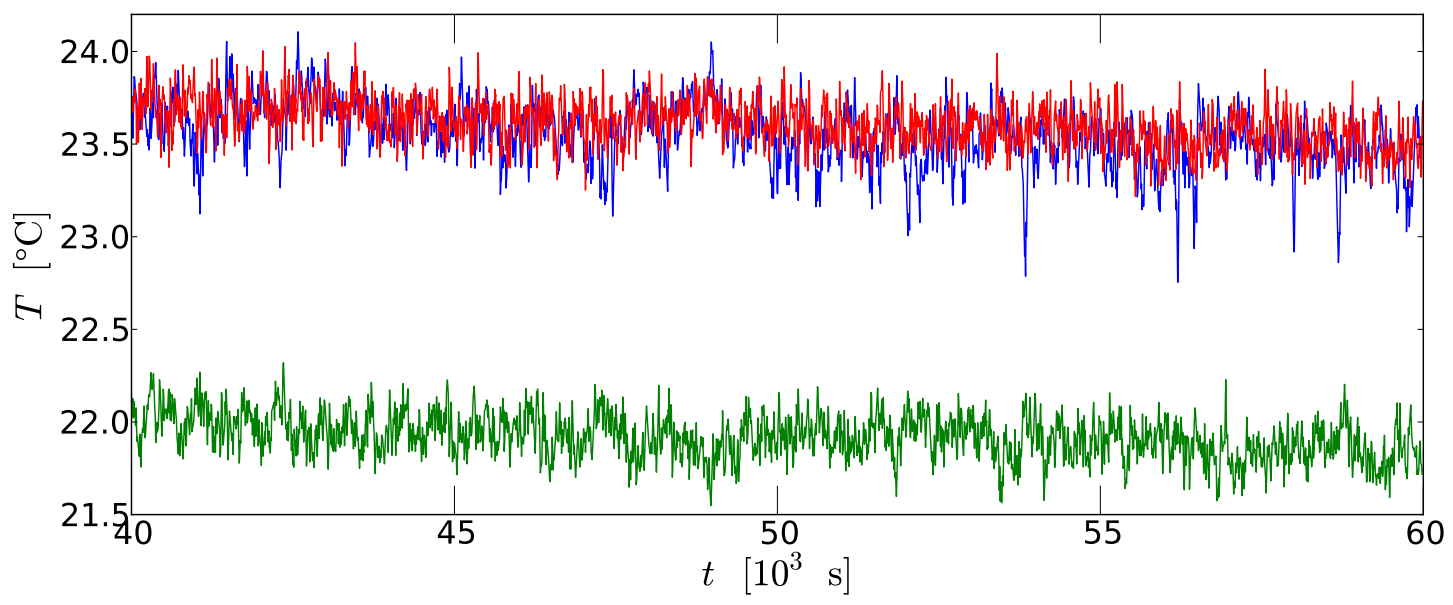

(a)

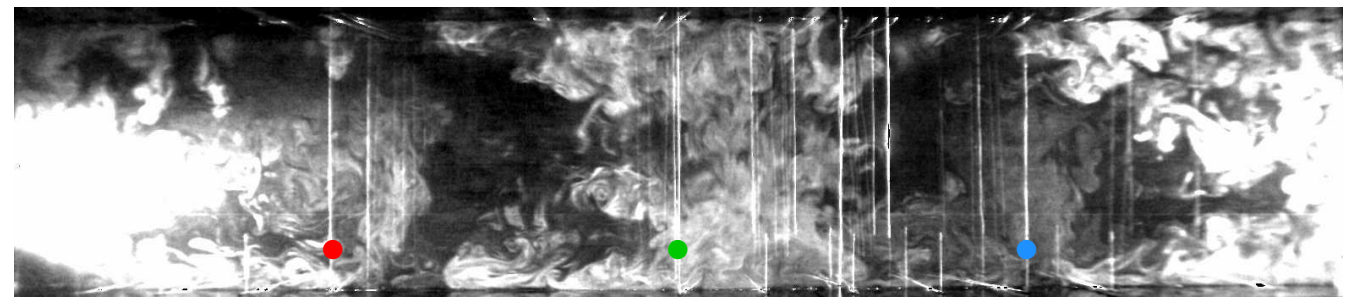

(b)

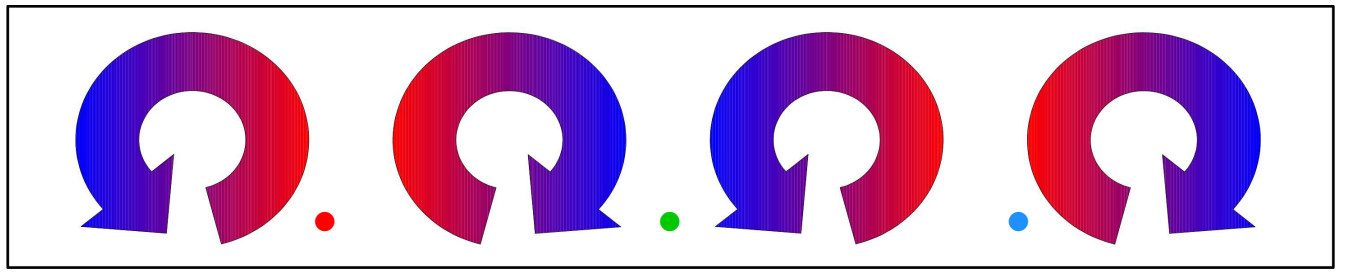

(c)

Figure 6.5: (a) Temperature time series at three selected sensor positions: P1 (red), P2 (green) and P3 (blue), see Table 6.2 and Figure 6.1. obtained for $A r=1.6, R a=1.6 \times 10^{8}$ and $R e=1.2 \times 10^{4}$. (b) Smoke visualisations corresponding to this temperature distribution, bullets mark the positions of P1, P2 and P3. A supplemental movie 1 of the smoke visualisation is available online [97. (c) Sketch of the LSCs showing four convection rolls.

The smoke images are taken, while smoke is injected homogeneously in the inflowing air. Thus, regions that appear bright are regions where the air descends, whereas darker regions with little smoke reflect zones of rising air. Totally, black areas are regions without smoke, 


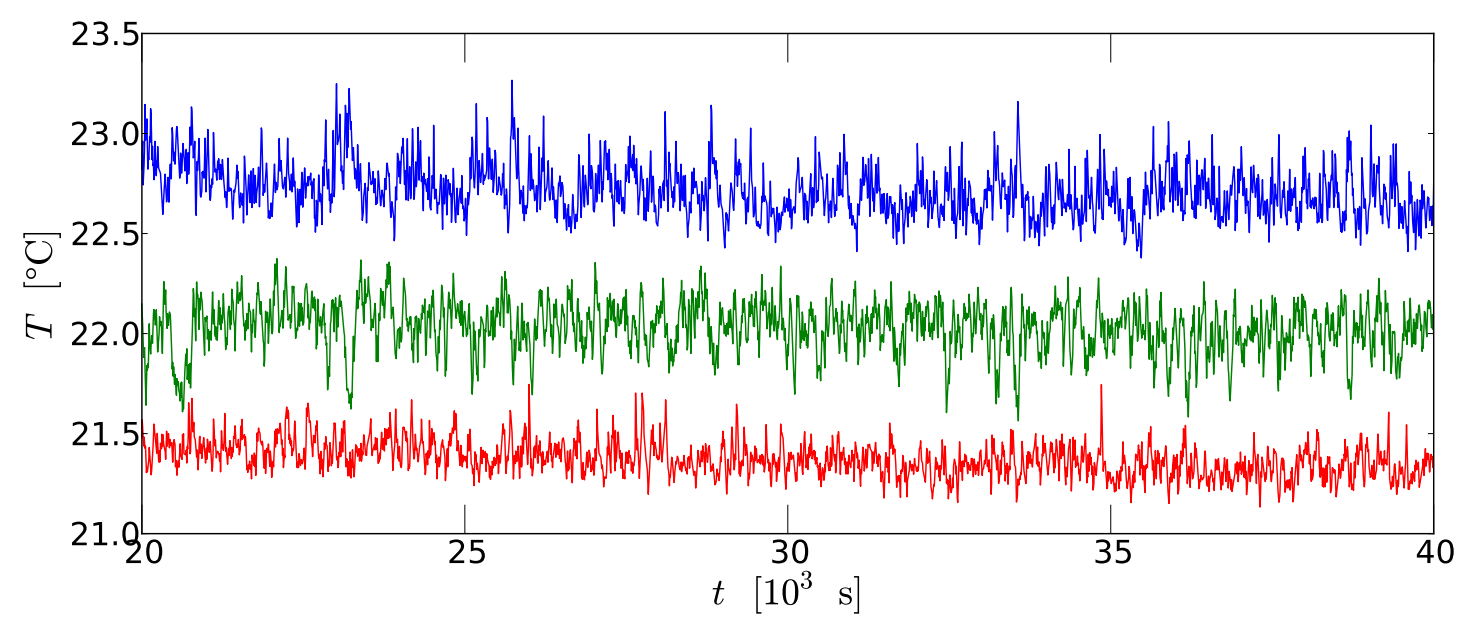

(a)

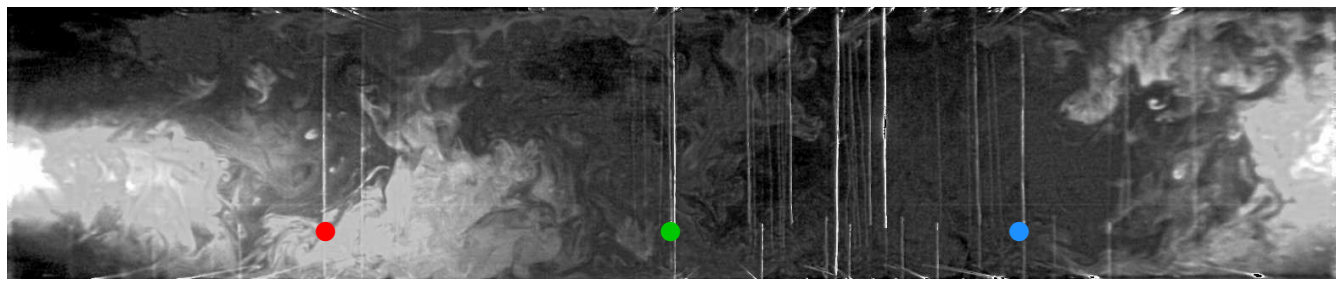

(b)

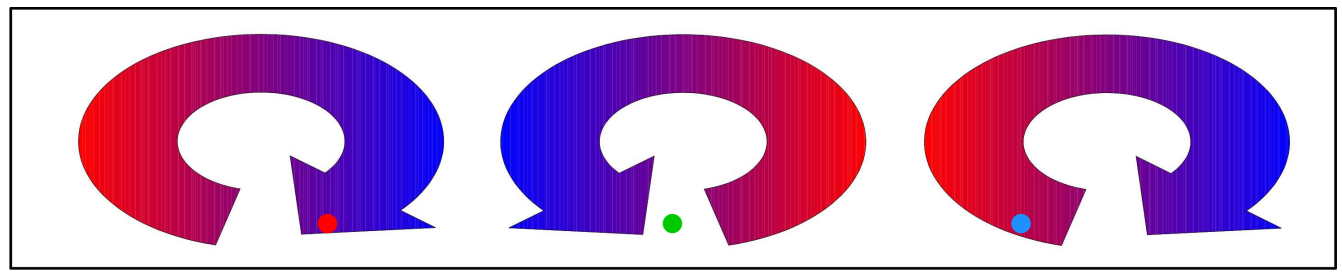

(c)

Figure 6.6: (a) Temperature time series at three selected sensor positions: P1 (red), P2 (green) and P3 (blue), see Table 6.2 and Figure 6.1. obtained for $A r=2.5, R a=1.1 \times 10^{8}$ and $R e=0.8 \times 10^{4}$. (b) Smoke visualisations corresponding to this temperature distribution, bullets mark the positions of P1, P2 and P3. A supplemental movie 2 of the smoke visualisation is available online 97. (c) Sketch of the LSCs showing three convection rolls.

for example, the centres of the convection rolls. The number of the rolls and the rotation direction of the LSCs are better visible in the supplemental movies available online [97].

Interpreting the smoke visualisations and taking into account geometric aspects of the experiments allows to ascribe warm regions to rising fluid and vice versa cold regions to downwelling fluid. The reason for this is that the heating plate is located just below the measurement plane (selected temperature sensors in $X-Y$ plane at constant $Z / H=$ 0.2 ). Thus, warm fluid originates from lower regions and is driven upwards by buoyancy. Additionally, considering the smoke visualisations allows to assign the flow structure, that 
is, the number and orientation of the LSCs to certain temperature distributions. The four rolls, visualised in Figure 6.5, are a result of cold air descending next to both side walls and in the centre region, while warm air rises at one and three-quarters of the cell length. Thus, the temperature at P2 (and close to both side walls) is lower compared to that at P1 and P3, where the warm air rises. In the three roll case, shown in Figure 6.6, P3 is closest to a region of rising warm fluid, and thus, P3 has the highest temperature. P1 is located close to a region of descending cold air, and therefore the temperature is the lowest there. The temperature level measured at P2 is located in between. This demonstrates that the measured mean temperature levels (Figure 6.5, a and Figure 6.6, a) reflect the vertical momentum transport in the LSC (Figure 6.5, b and Figure 6.6, b).

\subsubsection{TEMPERATURE TIME SERIES}

After the interpretation of the temperature distributions in terms of LSC configurations, we want to concentrate on different static and dynamic states which develop for certain $\operatorname{Re}-A r$ combinations. Therefore, Figure 6.7 gives an overview of the investigated parameter combinations and different observed LSC dynamics. We introduce the following three categories: stable LSCs (crosses), LSCs with some spontaneous reconfigurations (squares) and finally, LSCs with perfectly periodic oscillations (circles). Furthermore, for $R a>$ $3.0 \times 10^{8}$ and $R e>1.5 \times 10^{4}$ the LSCs are stable, that is, no oscillations and almost no spontaneous reversals occur.

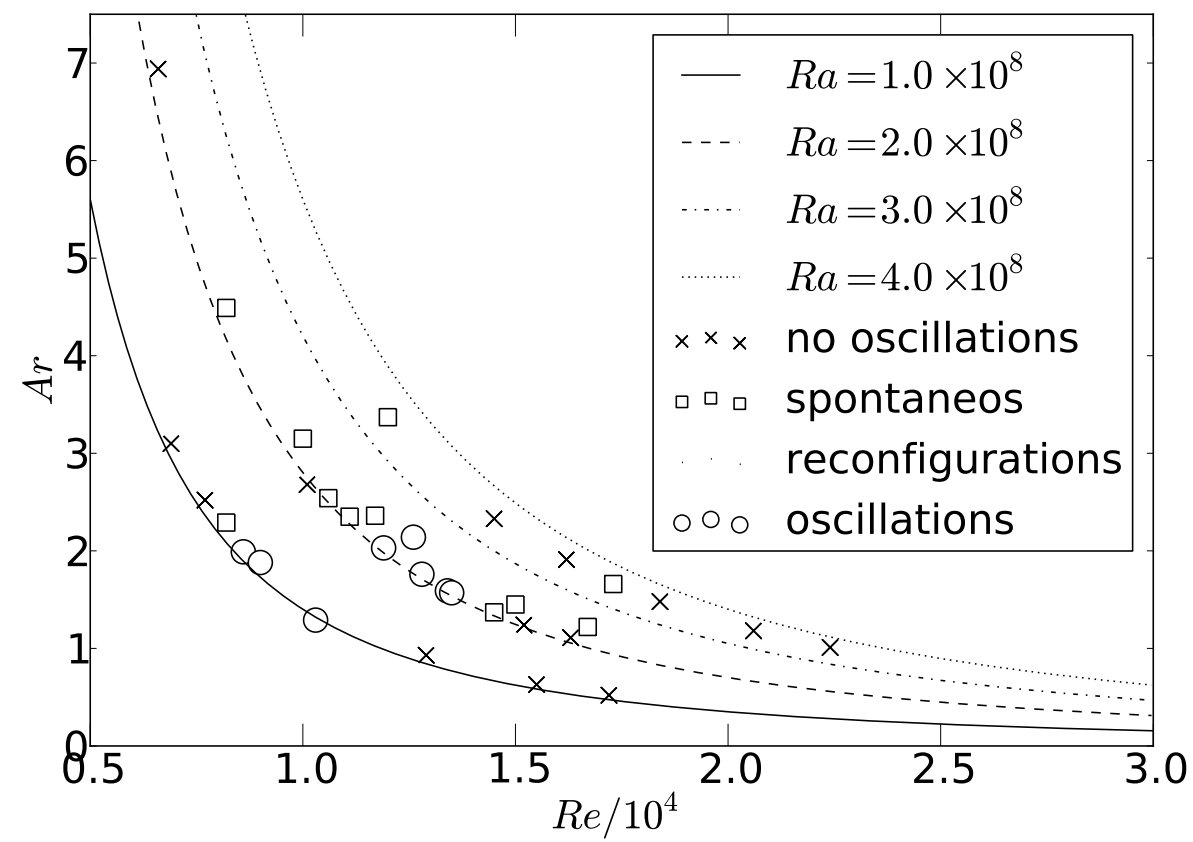

Figure 6.7: Investigated $R e-A r$ parameter space, lines indicate constant $R a$. Three different dynamical scenarios are found: steady configurations with no oscillations (crosses), spontaneous reconfigurations or quasi-periodic oscillations (squares) and periodic oscillations (circles). 
In the following, examples for the above introduced three categories are discussed for a fixed $R a$ of $2.0 \times 10^{8}$ and varying $R e$.

Temperature time series of a steady LSC, a LSC with a spontaneous reconfiguration and a LSC, which oscillates periodically, are presented in Figure 6.8 (a-c) for a time period of one hour. The temperature time series for $A r=1.1$ shown in Figure 6.8 (a) represents a LSC which was stable during the whole measurement time of about one day.

For $A r$ in one of the intermediate regimes between periodic oscillations and stable configurations, in our case $A r \approx 1.3$ or $A r \approx 2.3$, oscillations in the temperature signal occur sporadically. A temperature time series obtained for $A r \approx 2.3$ is presented in Figure 6.8 (b). Again, the temperature signals recorded at the above defined three sensor positions are plotted over a period of one hour. Most of the time, the temperature levels are maintained, but the values are different compared to $A r \approx 1.1$. However, the temperature decreases at P2 and increases at P1 aperiodically, then decreases at P3 and increases at P2. Afterwards, the temperature first decreases at P1 to its initial value before it decreases at P2 and increases at P3 to their respective initial values as well.

Figure 6.8 (c) shows temperature time series obtained for $A r=1.8$, revealing periodic oscillations of the temperature signals with a frequency of $f=2.0 \times 10^{-3} \mathrm{~s}^{-1}$, which were stable for the whole measurement time. The evolution process regarding the temperature signals of such an oscillation is exactly the same as in the case of $A r \approx 2.3$, where a single reorientation occurs (see Figure 6.8, b). It can be ascribed to a movement of the convection rolls to the right, also shown later in Figure 6.10,

Comparing the time series obtained for the three different $A r$ in Figure 6.8 reveals that for high or low $A r$ numbers (see also Figure 6.7), that is, ratios of buoyancy and inertia forces, the LSCs are stable. This means that small disturbances of the velocity or temperatures cannot change the arrangement of the found convection rolls. Further, for strong buoyancy forces (high $A r$ ), the rising warm air is not affected by the wall jet. It rather splits the wall jet, thus a steady LSC is formed in the whole cell. If, in contrast, the wall jet is strong (low $A r$ ), the warm upwelling fluid does not reach the top plate and steady TC-driven LSCs are formed within a FC dominated convection roll.

Analysing the signals of all temperature sensors in the layer $Z / H=0.2$ reveals four systematically different types of temperature distributions, which, taking into account our results of the smoke visualisations, reflect four different LSC arrangements: Two LSC configurations with three convection rolls (3R and 3Rc, see Figure 6.9, here c stands for counter rotating) and two with four convection rolls (4R and 4Rc, see Figure 6.9 develop during one oscillation period. Their temporal evolution is illustrated in Figure 6.9. A dynamic formation of the convection rolls at $X / L \approx 0$ (left side wall) and break down at $X / L \approx 1$ (right side wall) is also detected, resulting in the observed periodic change between LSC configurations with three and four rolls.

The detailed analysis of the oscillation process is given in Figure 6.10 (a-d). In addition, the corresponding temperature time series of P1, P2 and P3 are shown in Figure 6.11. which further indicate the points in time, at which the contours of Figure 6.10 are depicted. 


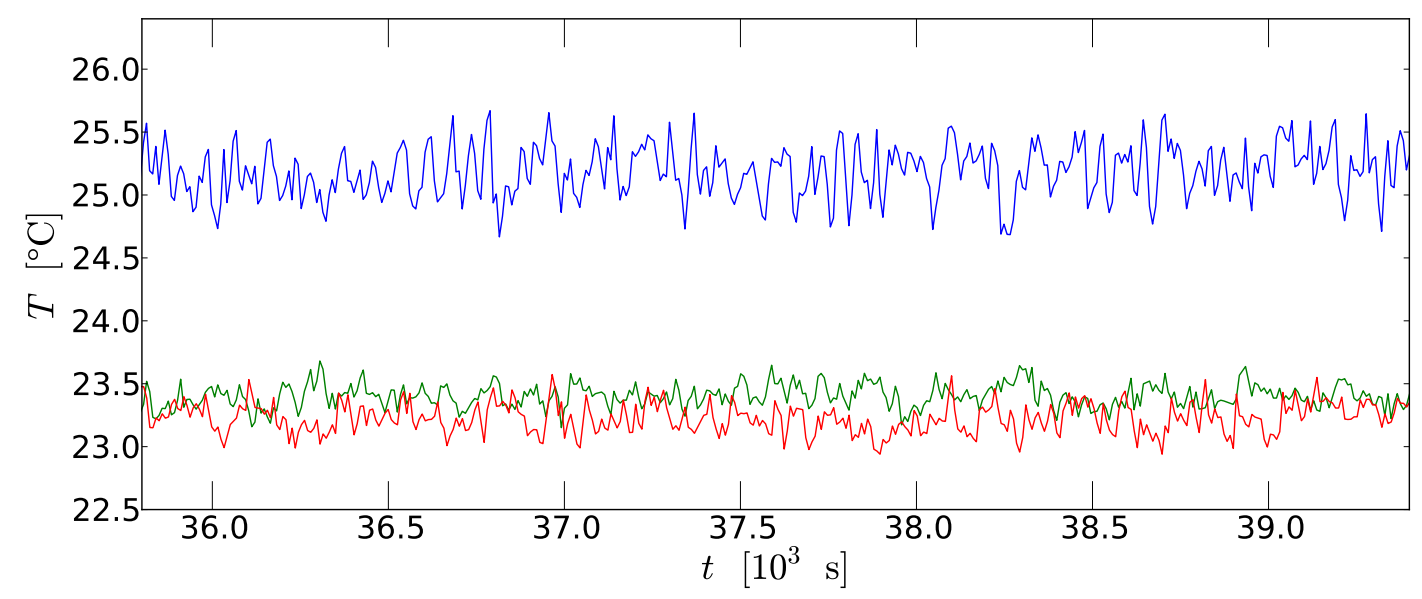

(a)

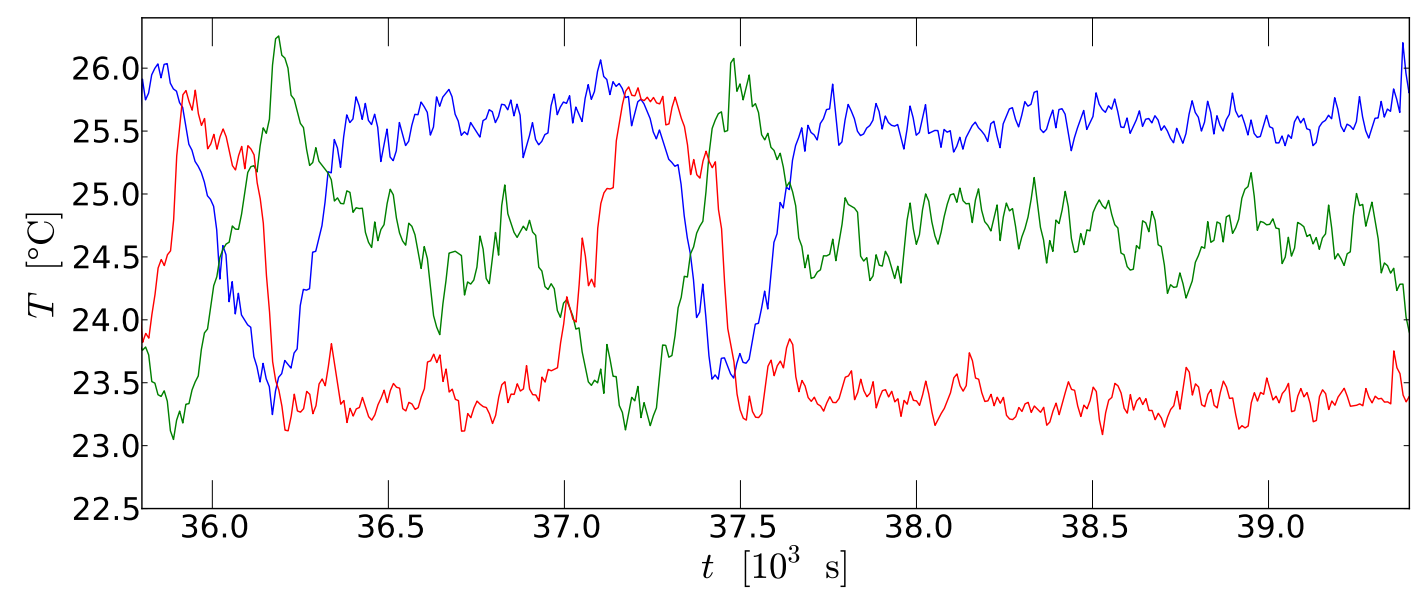

(b)

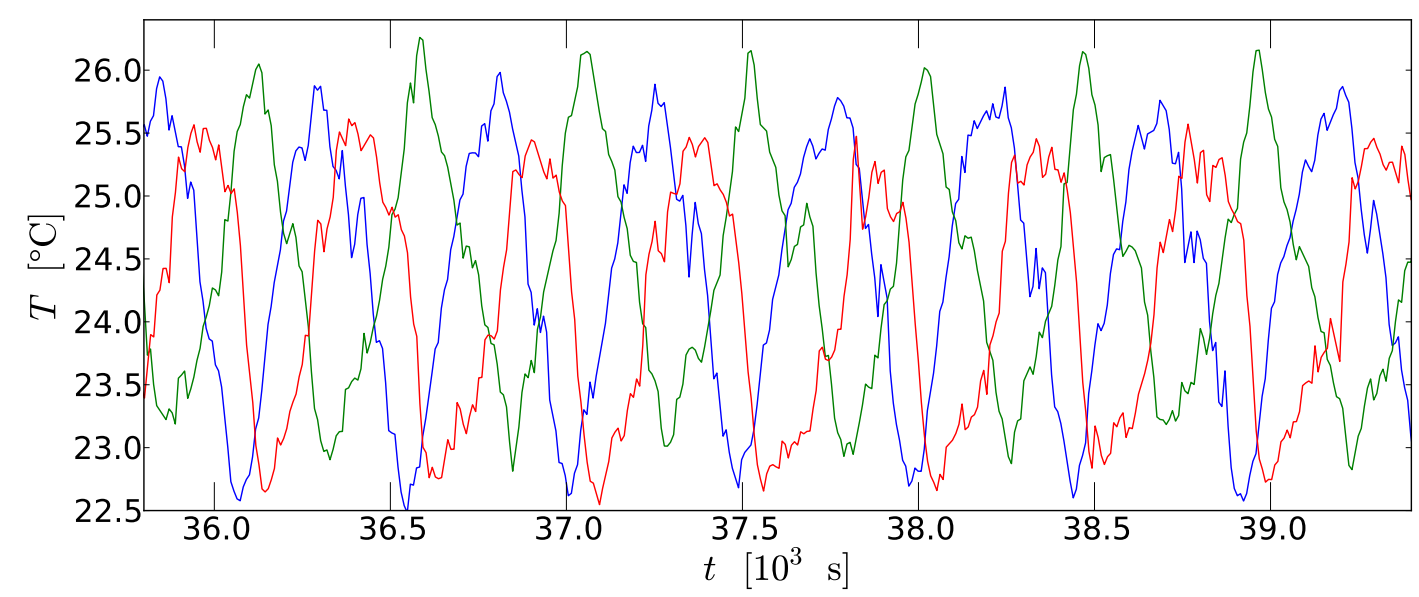

(c)

FiguRE 6.8: Temperature time series at three selected sensor positions: P1 (red), P2 (green) and P3 (blue), see Table 6.2 and Figure 6.1. At $R a=2.0 \times 10^{8}$ and (a) $A r=1.1$, (b) $A r=2.3$ and (c) $A r=1.8$. 


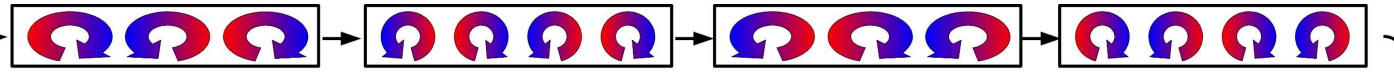 \\ $3 R$ \\ $4 \mathrm{R}$ \\ $3 R c$ \\ $4 \mathrm{Rc}$}

FiguRE 6.9: Sketch of the dynamics of the convection rolls. The pictograms, each showing the $X-Z$ plane, correspond from left to right to the temperature distributions (a) to (d) in Figure 6.10.

The four images in Figure 6.10 (a-d) are instantaneous temperature contour plots in a layer parallel to the heating plate at $Z / H=0.2$, which are spaced equidistantly in time over one oscillation period. As can be seen, the temperature in the rear part of the cell (higher $Y$ ) is always higher than in the front part for a given $X$ position. This can be explained by the fact that the cold air which enters the cell through the inlet slot at the cooling plate first follows the ceiling and down the front wall before it moves in positive $Y$ direction over the bottom plate. Above the heating plate, it warms up, and thus, the rear is warmer than the front for a given $X$ position. At high $A r$ (not shown here for the sake of brevity), it also happens that the inflowing air cannot follow the ceiling, but descents immediately after entering the cell. In these cases, the cold air reaches the bottom plate in the rear part and flows in negative $Y$ direction over the bottom. Hence, the rear is colder than the front part of the cell at a given $X$. Anyhow, in the considered case shown in Figure 6.10 , the inertia forces are strong enough to prevent such a bypass flow and to superimpose a three-dimensional forced convection roll.

The considerations in the Section 6.3.2 aiming to correlate the temperature distributions with a certain LSC configuration allow to assign the convection rolls in Figure 6.9 to the temperature distributions in Figure 6.10. Since the four different temperature distributions occur periodically, we conclude that there is a continuous change of the number of convection rolls between three and four with different rotational directions, adding up to the above discussed four different LSC configurations.

The arrows in Figure 6.10 indicate the movement of warm or cold air during one oscillation. By analysing the travelled distance of a certain region in a given period of time, the travelling velocity $u$ in $X$ direction is determined to amount $u=\frac{\Delta X}{\Delta t_{\mathrm{osc}}}=\frac{1.25 \mathrm{~m}}{500 \mathrm{~s}}=2.5 \times 10^{-3} \mathrm{~ms}^{-1}$. $u$ is a measure for the speed of the LSC movement towards the right side wall. It is two orders of magnitude lower than the inflow, the buoyancy and the horizontal velocity component of the TC-induced LSC [105] $u_{\text {in }}=0.40 \mathrm{~ms}^{-1}, u_{\text {buoy }}=\sqrt{\beta g H \Delta T}=0.56 \mathrm{~ms}^{-1}$ and $u_{\mathrm{LSC}(\mathrm{TC})}=0.11 \mathrm{~ms}^{-1}$ for this case, respectively. On the other hand, $u$ is two orders of magnitude higher than the thermal diffusion velocity $u_{\kappa} \approx \frac{\kappa}{H}=4.3 \times 10^{-5} \mathrm{~ms}^{-1}$. For further comparison, we estimated the eddy turnover frequencies of the FC roll and the buoyancy-induced LSCs using the mean inflow velocity and the horizontal velocity component of the LSC [105], respectively. The resulting frequencies $f_{F C} \approx 2 \times 10^{-1} \mathrm{~s}^{-1}$ and $f_{L S C s} \approx 4.2 \times 10^{-2} \mathrm{~s}^{-1}$ are at least 20 times higher than our observed frequency of $f=1 / \Delta t=2 \times 10^{-3} \mathrm{~s}^{-1}$.

Additionally, we know from tomographic PIV, which was performed in our sample for 


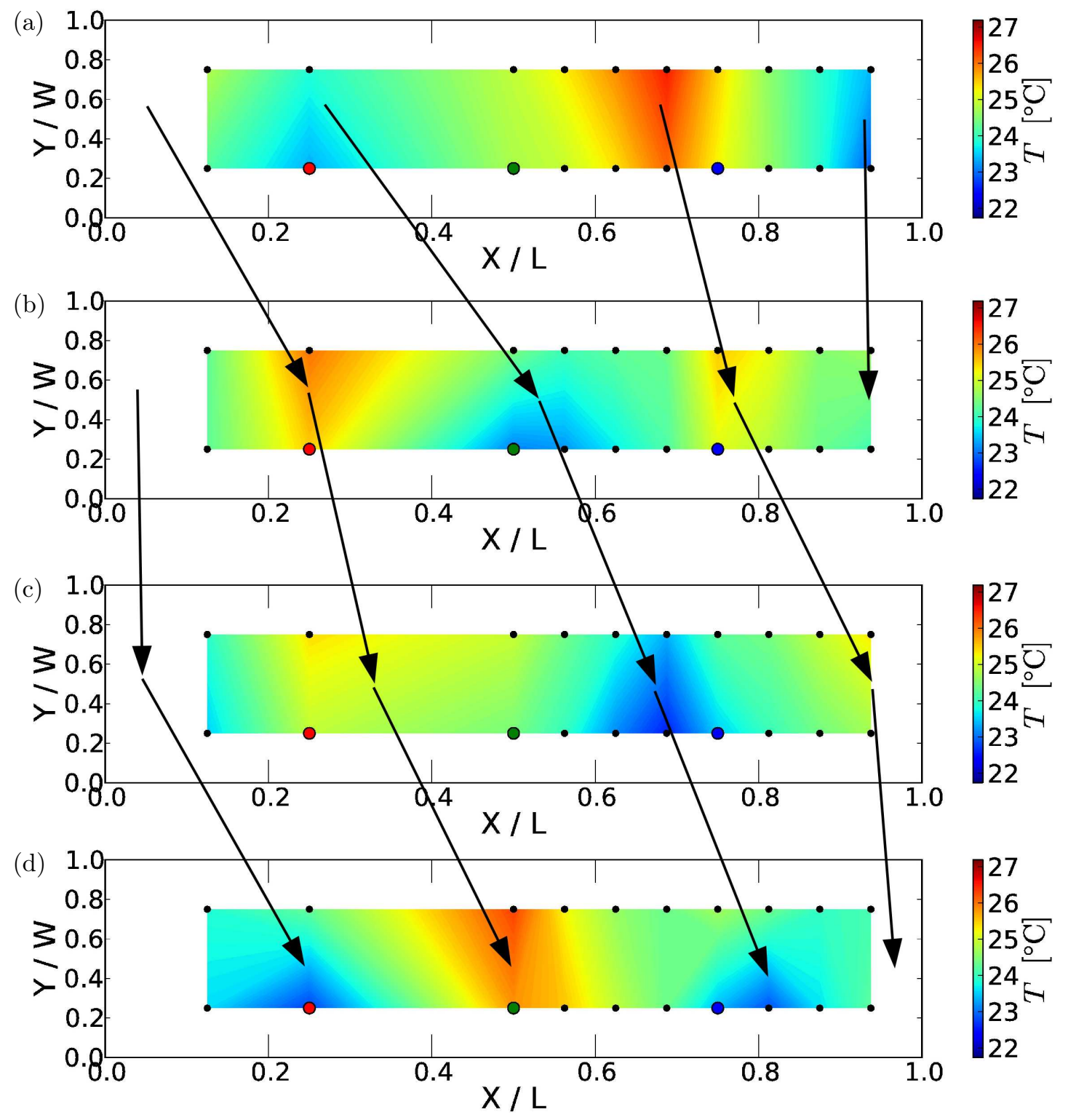

Figure 6.10: 2D instantaneous temperature distributions in the layer $Z / H=0.20$ at $A r=1.8$ and $R a=2.0 \times 10^{8}$. Temperatures were measured at the positions which are marked as black and colourful dots. Time lag between two sequent images is exactly one-fourth of the oscillation period (see also Figure 6.11. The temperature profiles can be assigned to different roll configurations: (a) 3R, (b) 4R, (c) 3Rc and (d) 4Rc (see Figure 6.9p. A supplemental movie 3 made of many of these instantaneous temperature distributions is available online 97$]$.

$R e=1.0 \times 10^{4}$ and $A r=3.6$ (i.e. $R a=2.56 \times 10^{8}$ ) that four TC-induced LSCs arrange in the box in a "W"-shape 67]. This alignment with cold air descending at three positions in the front of the cell and warm air rising at two positions in the rear of the cell corresponds to the instantaneous temperature distribution shown in Figure6.10 (b). During the rather short measurement time realised for the tomographic analysis of approximately $750 \mathrm{~s}$, however, no reorientations took place. The mean velocity and the length of one LSC are 


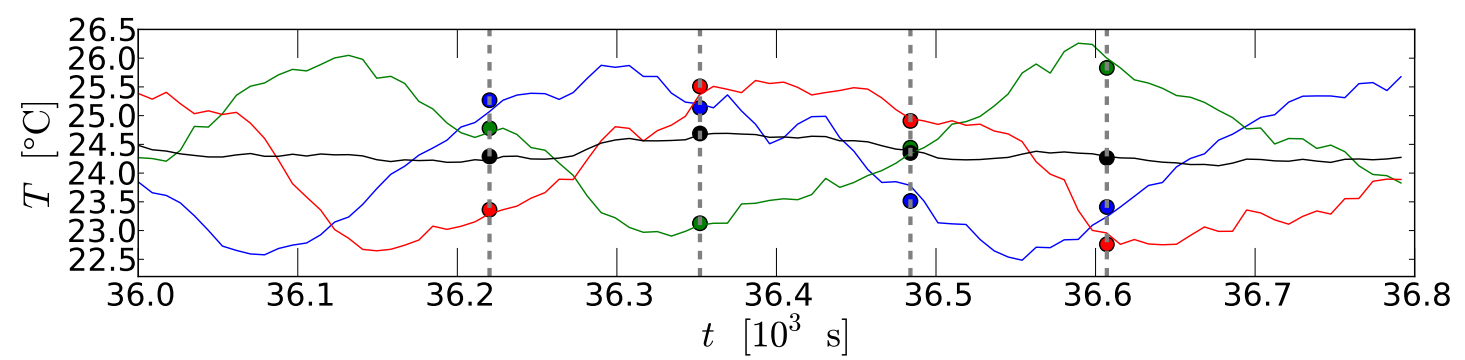

Figure 6.11: Temperature time series at three selected sensor positions: P1 (red), P2 (green) and P3 (blue), see Figure 6.10 as well as Table 6.2 and Figure 6.1, and mean fluid temperature in the cell. The four marked points in time correspond to the instantaneous temperature distributions in Figure 6.10, (a) to (d), from left to right.

estimated to $|u| \approx 0.09 \mathrm{~ms}^{-1}$ and $L_{\mathrm{LSC}} \approx 2 \times 0.8 \mathrm{~m}+2 \times 0.5 \mathrm{~m}=2.6 \mathrm{~m}$ (see Figure 6.12 , $\mathrm{b}$ and $\mathrm{c}$ ), which leads to an eddy turnover frequency of $f_{\mathrm{LSC}} \approx 3.4 \times 10^{-2} \mathrm{~s}^{-1}$. This is in good agreement with the value $f_{L S C} \approx 4.2 \times 10^{-2} \mathrm{~s}^{-1}$ estimated above, even though the measurements were conducted at slightly different $R e, R a$ and $A r$.

Table 6.4 summarises the estimated and measured velocities and frequencies at $R a=$ $2.0 \times 10^{8}$ and $A r=1.8$.

TABLE 6.4: Comparison of the velocities and frequencies at $R a=2.0 \times 10^{8}$ and $A r=1.8$, bold velocities are measured, others are estimated.

\begin{tabular}{|c|c|c|c|}
\hline Origin & $\begin{array}{l}\text { Length } \\
(\mathrm{m})\end{array}$ & $\begin{array}{l}\text { Velocity } \\
\left(\mathrm{ms}^{-1}\right)\end{array}$ & $\begin{array}{l}\text { Frequency } \\
\left(\mathrm{s}^{-1}\right)\end{array}$ \\
\hline $\mathrm{FC}$ & 2.0, see Figure 6.12 (a) & 0.40 & 0.20 \\
\hline buoyancy & 2.6 , see Figure 6.12 (b and c) & 0.56 & 0.22 \\
\hline LSC (TC induced) & 2.6, see Figure $6.12(\mathrm{~b}$ and $\mathrm{c})$ & 0.11 & $4.2 \times 10^{-2}$ \\
\hline $\begin{array}{l}\text { diffusion (purely } \\
\text { conductive) }\end{array}$ & 0.5 (height of the cell) & $4.3 \times 10^{-5}$ & $8.6 \times 10^{-5}$ \\
\hline here observed & 1.25 , see Figure 6.10 & $2.5 \times 10^{-3}$ & $2 \times 10^{-3}$ \\
\hline
\end{tabular}

The fact that the dynamic processes of the roll formation and break down lead to a continuous change of the LSC configuration indicates the coupling between the side walls. A detailed analysis of the time scale of the TC-induced LSCs 105 reveals that a convected distortion needs approximately $\Delta t_{\mathrm{LSC}}=\frac{8 \times 0.5 \mathrm{~m}+8 \times 0.8 \mathrm{~m}}{u_{\mathrm{LSC}(\mathrm{TC})}} \approx 95 \mathrm{~s}$ to travel from one side wall to the other and back. Here, we assumed the travelled path to follow a four roll configuration, see Figure 6.13. Considering that the rolls arrange in a "W"-shape as mentioned above, the side lengths of each roll are estimated to $0.5 \mathrm{~m}$ and $0.8 \mathrm{~m}$. The time scale is approximately a quarter of the observed oscillation period $\frac{\Delta t_{\text {osc }}}{4} \approx 125 \mathrm{~s}$, which corresponds to the time that the system needs to change from a configuration to the next one, for example, from $3 \mathrm{R}$ to $4 \mathrm{R}$. This estimated time scale reflects the fastest possible way to transport information 
with the LSCs assuming that the dynamics at the two opposing side walls are coupled.

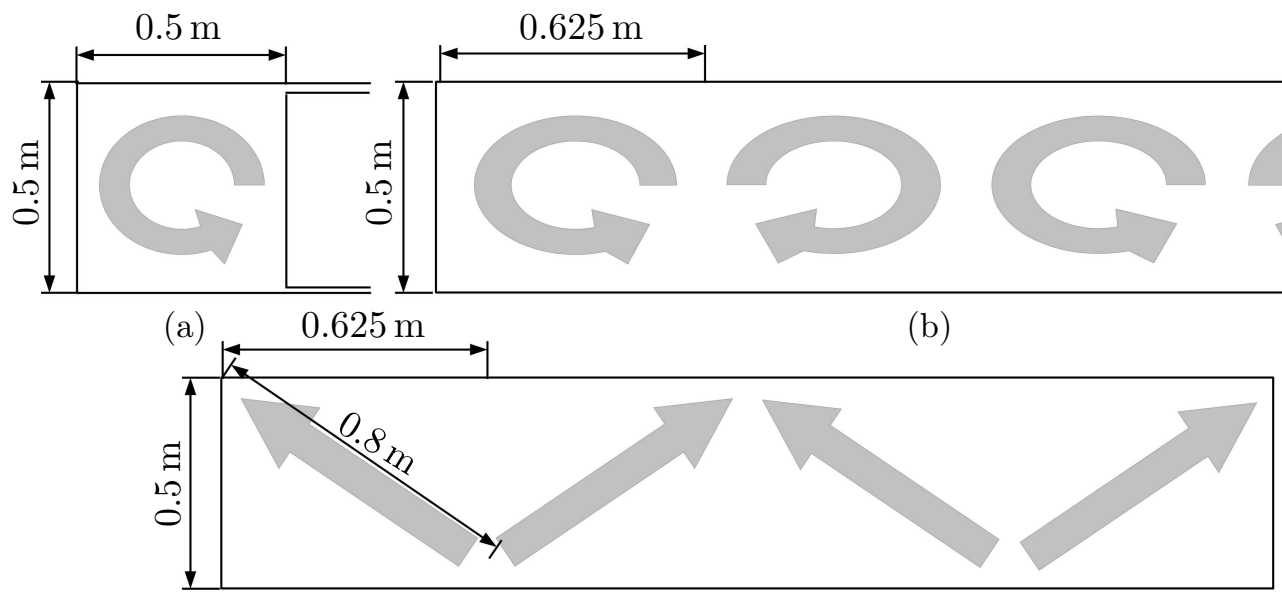

(c)

Figure 6.12: Sketch of the length of the convection rolls, (a) in the $y-z$ plane for FC, (b) in the $x-z$ plane for TC for a four roll configurations and (c) top view ( $x-y$ plane) on a layer close above the heating plate for $\mathrm{TC}$ in a four roll configurations.

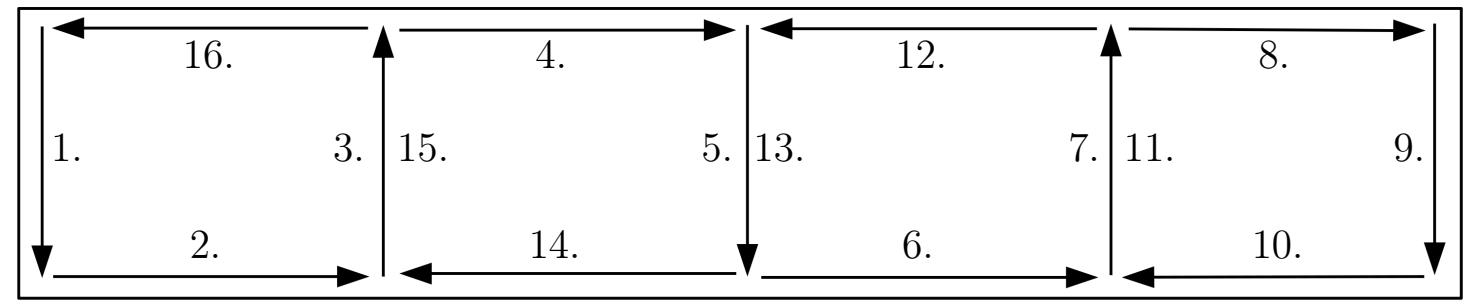

Figure 6.13: Sketch of the travelled path in the $x-z$ plane of a distortion starting in the top left corner for a four roll configurations.

\subsubsection{TWO-POINT CORRELATIONS}

We present in this subsection two-point correlations in phase diagrams of the temperature signals recorded at P1 and P3. These diagrams help to characterise the dynamical behaviour of the different observed scenarios: steady LSCs, LSCs with spontaneous reconfigurations and periodically oscillating cases.

Figure 6.14 shows phase diagrams for four different $A r$ and at a fixed $R a$ of $2.0 \times 10^{8}$. Thereby, (a)-(c) correspond to the temperature time series presented in Figure6.8 (a)-(c). Further, the phase diagram of an additional $A r$ at which a steady LSCs showed very few reconfigurations compared to those illustrated in Figure 6.14 (b) is presented in Figure 6.14 (d).

For the stable LSCs, (a) in Figure 6.8 and Figure 6.14, a single fixed point in the $T_{\mathrm{P} 3}-T_{\mathrm{P} 1}$ phase space exists. The temperatures at P3 and P1 amount to approximately 25 and $23.2^{\circ} \mathrm{C}$, respectively, and vary only slightly during the measurement time. In contrast, the behaviour in the phase diagrams is significantly different for cases with spontaneous 


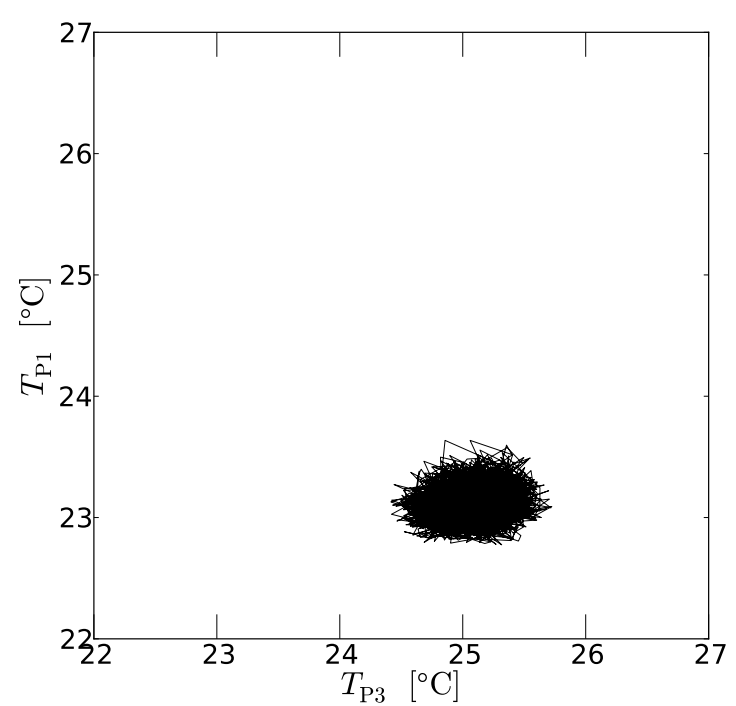

(a)

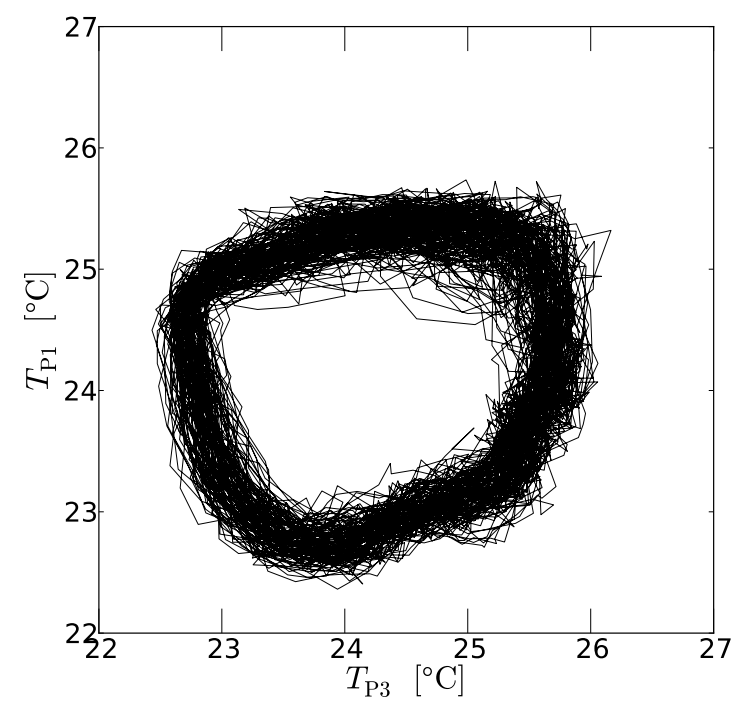

(c)

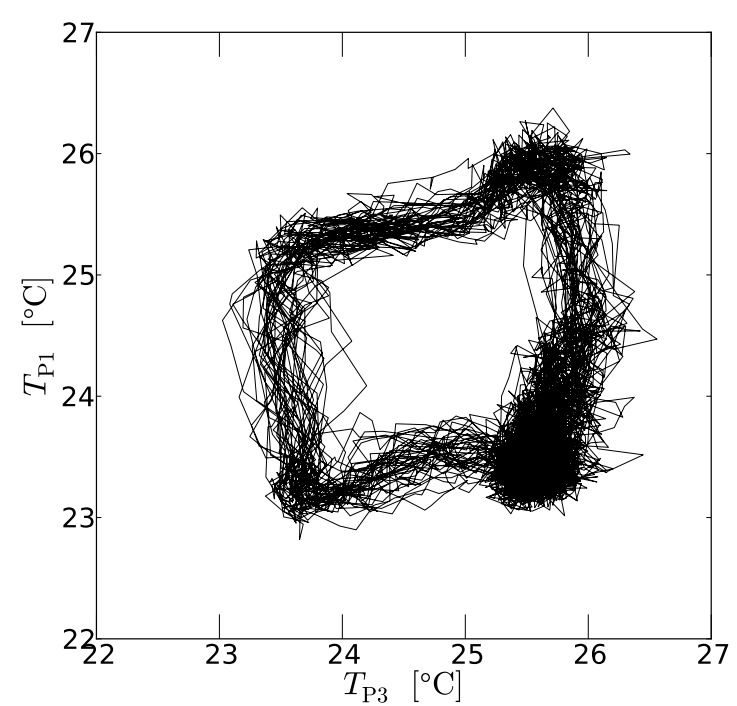

(b)

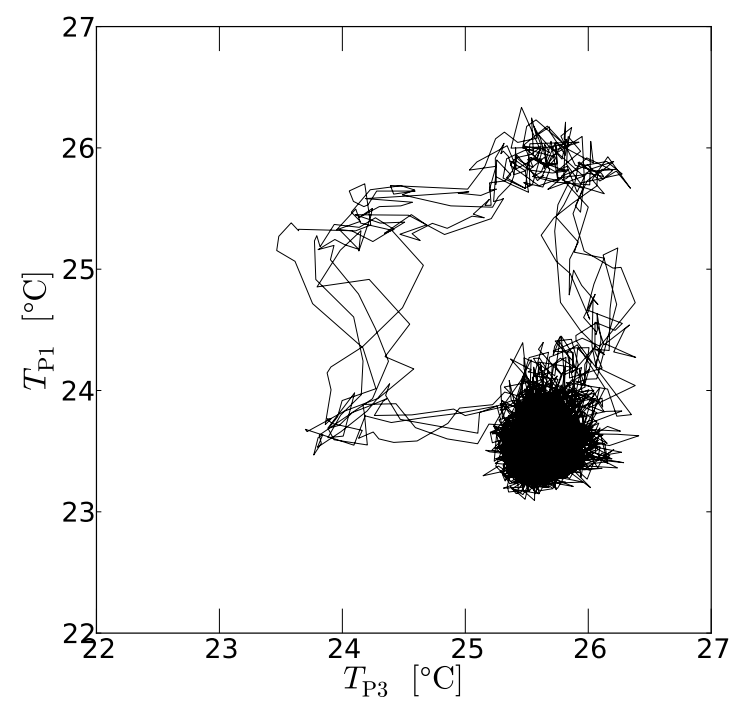

(d)

Figure 6.14: Temperature phase diagrams at P3 and P1 at $R a=2.0 \times 10^{8}$. (a) $A r=1.1$, (b) $A r=2.3$, (c) $A r=1.8$ and (d) $A r=2.5$.

reconfigurations, see Figure 6.14 (b) and (d), where the data of (b) correspond to Figure 6.8 (b). Thereby, (b) is a parameter combination with many spontaneous reconfigurations, whereas (d) reveals only very few reconfigurations. Again the system is most likely to be found in a LSC configuration with constant $T_{\mathrm{P} 3} \approx 25.5^{\circ} \mathrm{C}$ and $T_{\mathrm{P} 1} \approx 23.5^{\circ} \mathrm{C}$, however, also describes some paths in the phase diagram. These paths reveal some interesting features. Firstly, they are almost squares, which means that the temperature changes at one position, either P1 or P3, while it stays constant at the other. Secondly, a slight distortion of the square is found in the top right corner. A possible explanation is that the LSCs arrange a bit longer in a configuration with warm regions at P1 and P3, before rearranging again in 
different configurations.

For the oscillating case, see (c) in Figure 6.8 and Figure 6.14, there is no fixed point, that is, no most likely appearing configuration of the LSCs. The shape of the path in the phase diagram is a mixture of a square and a circle, that is, a mixture between a system with constant temperature at one point while it changes at the other (square) and a system with temperature values changing sinusoidal with a phase shift of $\pi / 2$ between the different points (circle). Though, their temperature time dependency can be described as a superposition of a sinusoidal and an in phase square function. Furthermore, the temperatures at P1 and P3 are strongly correlated, and their functions have a phase shift of approximately $\pi / 2$.

\subsubsection{Frequency ANALYSiS}

Fast Fourier transformations of the temperature time series lead to the normalised frequency spectra for $A r=1.8$ and $R a=2.0 \times 10^{8}$ presented in Figure 6.15. Here, (a) depicts the power spectrum of the temperature time series measured at P2 (see Table 6.2 Figure 6.1), while (b) shows the power spectrum of $A r$. Regarding the fluid temperature (see Figure 6.15, a), the frequency $f=2 \times 10^{-3} \mathrm{~Hz}$ dominates the spectrum, with a peak value three orders of magnitude higher than that of the other frequencies. Additionally, peaks occur in the spectrum of the sensor signal at P2 at multiple frequencies $\left(f=4 \times 10^{-3} \mathrm{~Hz}\right.$ and $f=6 \times 10^{-3} \mathrm{~Hz}$ ). In contrast, in the spectrum of $A r$ (see Figure 6.15, b), which is a function of the inflow velocity $(R e)$ and the temperature difference $(R a)$, no dominant frequency is visible. This confirms that fluid temperature oscillations characterised by a single dominating main frequency are not triggered by variations in the boundary conditions (here: inflow velocity and temperature difference). Thus, they are triggered by internal flow-induced mechanisms.

Figure 6.16 illustrates how the main oscillation frequencies depend on $R e$ and $A r$ for two different $R a$ numbers (open symbols: $R a=1.1 \times 10^{8}$ and filled symbols: $R a=2.0 \times 10^{8}$ ). The different symbols indicate the dynamics of the LSCs: steady configurations with no oscillations (triangles), spontaneous reconfigurations or quasi-periodic oscillations (squares) and periodic oscillations (circles). The dependency of the frequency on Re presented in Figure 6.16 (a) underlines the linear dependency, as indicated by the regression line, of the oscillation frequency on $R e$ for cases with periodic oscillations. The frequency scales with $f\left[10^{-3} \mathrm{~s}^{-1}\right] \sim 2.1 \times \operatorname{Re}\left[10^{4}\right]$ and, thus, with the inflow velocity. On the other hand, periodic oscillations occur only for $R a<3.0 \times 10^{8}$ (see Figure 6.7) which indicates that the TC contribution determines whether oscillations can occur or not. This result is in good agreement with observations in the literature [124], who did not find any reversals in pure TC for $R a \geq 4 \times 10^{7}$ for $\operatorname{Pr} \approx 0.7$. The deviation of the absolute numbers will be caused by the different geometries and the different systems, that is, MC versus TC. Furthermore, it is known that an increase in the mean wind velocity leads to thinner thermal boundary layers and thus to a higher effective $R a$ number. 


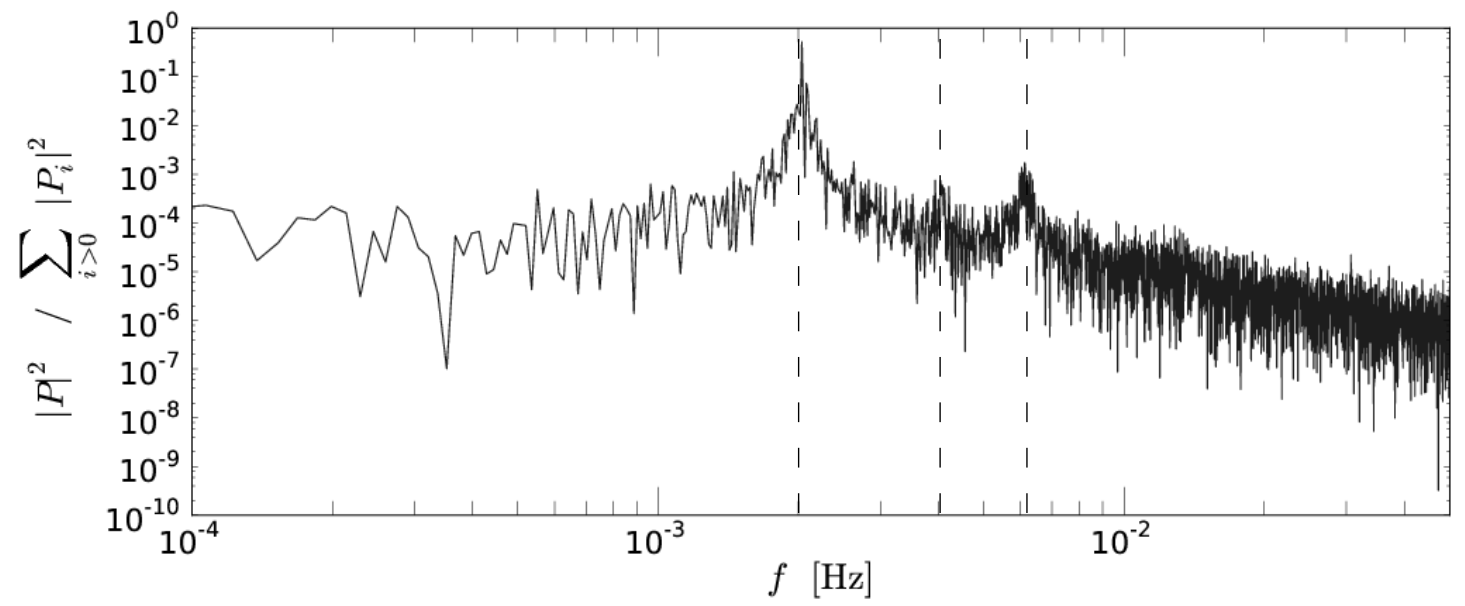

(a)

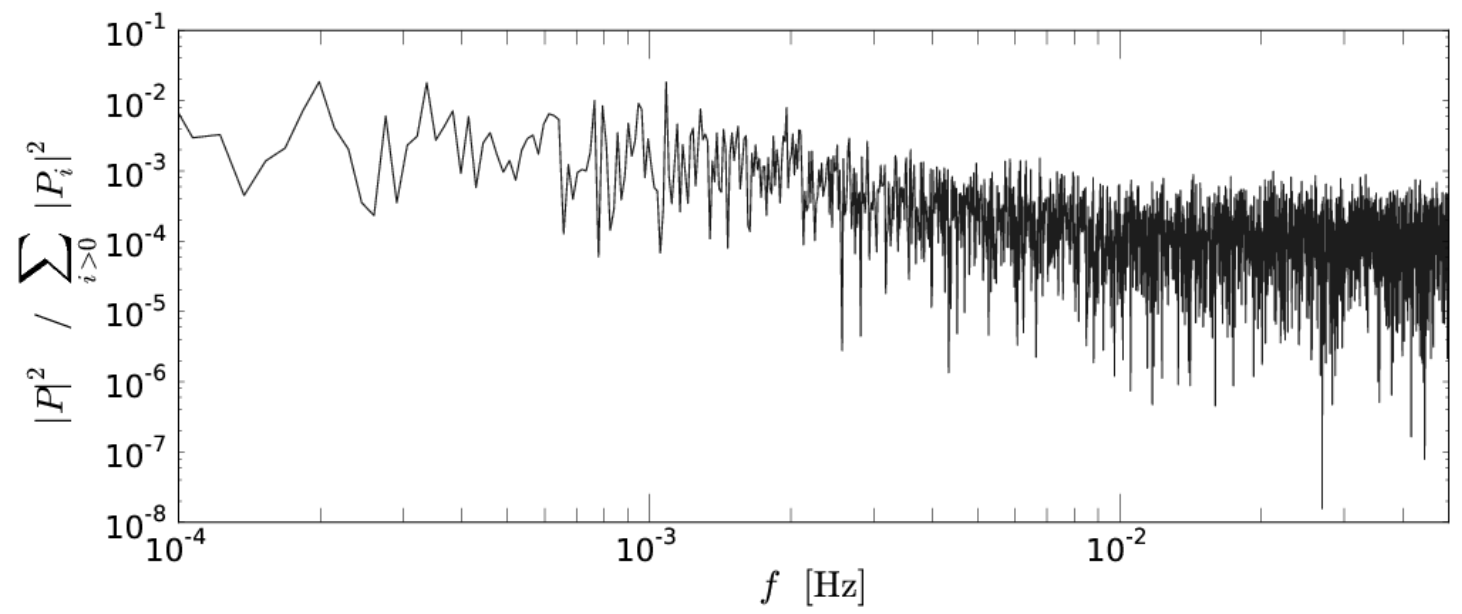

(b)

FiguRe 6.15: Normalised frequency spectra on double logarithmic scale at $R e=1.3 \times 10^{4}$, $A r=1.8$ and $R a=2.0 \times 10^{8}$ (a) of temperature time series measured at P2, (b) of $A r$.

Since the temperatures of the ceiling and the inflowing air slightly differ, we calculated an effective Rayleigh number $R a_{\text {eff }}$. The latter is based on the temperature difference between the heated bottom and the inflowing air ( $\left.\Delta T_{\text {bottom-inflow }}\right)$ in contrast to the $R a$ number used above which is determined with the temperature difference between bottom and ceiling ( $\Delta T_{\text {bottom-ceiling }}$ ). Using $R a_{\text {eff }}$ instead of $R a$ leads to an effective Archimedes number $A r_{\text {eff }}$ as well. In Figure 6.16 (b), we show the oscillation frequency as a function of $A r_{\text {eff }}$. By using $A r_{\text {eff }}$, the regions separating the different dynamics collapse for the different $R a$. We therefore conclude, that $A r_{\text {eff }}$ is better suited to distinguish oscillating flow states from steady ones. For high and low $A r_{\text {eff }}$, that is, region III, the LSCs are stable (within our measurement time). The reason might be that either buoyancy or inertia forces are dominant. In the intermediate range, that is, $1.5<A r_{\text {eff }}<3.2$, the LSCs are not stable. 


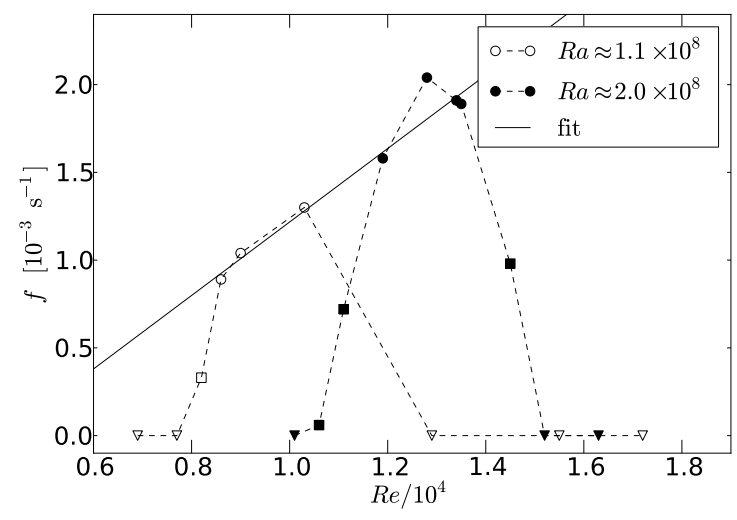

(a)

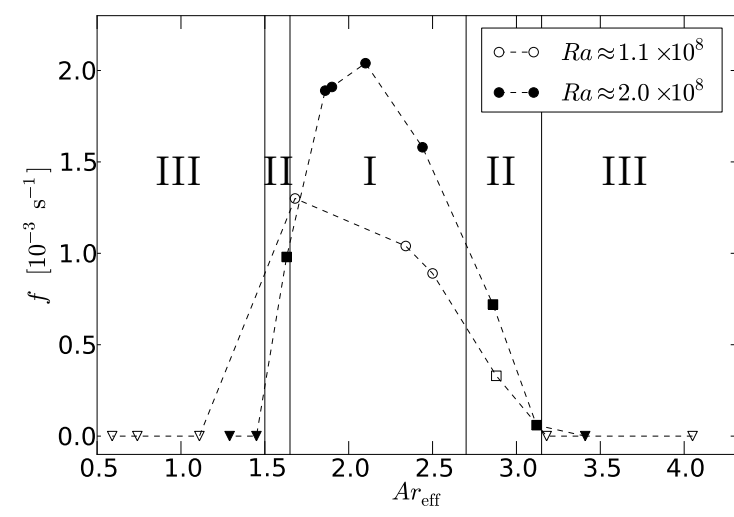

(b)

Figure 6.16: Main frequencies of the recorded temperature oscillations. Different symbols indicate: steady configurations with no oscillations (triangles), spontaneous reconfigurations or quasi-periodic oscillations (squares) and periodic oscillations (circles). Open symbols: $R a \approx 1.1 \times 10^{8}$ and filled symbols: $R a \approx 2.0 \times 10^{8}$. (a) Frequencies as a function of $R e$. (b) Frequencies as a function of $A r_{\text {eff }}$. The region classifications stand for: periodic oscillations (I), spontaneous reconfigurations (II), here the frequency is the number of events divided by the measurement time and finally no oscillations/steady LSC configurations (III).

Thereby, periodic oscillations were found for $1.6<A r_{\text {eff }}<2.7$, that is, region I, whereas in the other $A r_{\text {eff }}$ regions (II) we observed spontaneous reconfigurations or "jumps". For cases with spontaneous reconfigurations, the measured frequency is a result of the number of events divided by the total measuring time. Finally, it must be noted that at $A r_{\text {eff }}=2.1$ $\left(R a=2.0 \times 10^{8}\right)$, the frequency reaches its maximum value of $f=2.0 \times 10^{-3} \mathrm{~s}^{-1}$.

These different dynamical modes as well as their dependency on $A r\left(A r_{\text {eff }}\right)$ are also shown in the phase spaces depicted in Figure 6.14. With increasing $A r$ (a-c-b-d in Figure 6.14), the LSC configuration is stable (a), periodically oscillating (highly instable) (c), rather instable with a fixed point (b) and almost stable with only very few reconfigurations (d), well corresponding to Figure 6.16 (b).

To summarise the discussion of this section we state that for fixed $\operatorname{Pr} \approx 0.7$ :

1. The strength of TC $(R a)$ determines if oscillations of the LSCs can occur

2. $\mathrm{MC}\left(A r_{\text {eff }}\right)$ determines the dynamics (steady, spontaneous reconfigurations or periodic oscillations) if TC allows oscillations

3. FC $(R e)$ determines the oscillation frequency if periodic oscillations occur

The analysis further revealed that whether stable or periodically oscillating LSCs develop can be controlled by adjusting the inflow velocity, that is, Re. In the future, this sensitivity might allow for a control of the LSCs in rooms or aircraft cabins.

As a further comment, it should be noted that there is an upper boundary for $\operatorname{Pr}$ in pure TC, above which no reversals occur anymore [124. In their explanation, the existence 
of the bound is related to the boundary layer thicknesses. For both, too large and too small ratio of thermal to kinetic boundary layer thickness, the transport of thermal energy towards the corner rolls is damped, and thus, no reversals can occur. To our knowledge, the $\operatorname{Pr}$ dependency has not been analysed in MC yet.

\subsubsection{DynamiCs OF THE LSCS}

During the transition between flow states with three and four LSCs, rolls develop and break down again. However, this never happened in the cell centre. The LSCs always developed and collapsed at one of the short side walls. That a LSC never breaks down in the centre of the cell can be easily explained by looking at the rotation directions of the remaining LSCs. These would not fit if only one centre LSC broke down, thus two centre LSCs have to break down simultaneously. However, the possibility for such a double break down is lower than that of a to a single roll break down because of a higher potential barrier for the change from the one configuration to the other. The principle of potential barriers which separate different LSC configurations was introduced for pure RBC, see for example, 118 or 23. Whereas Sreenivasan et al. 118 only describe the reversals of the LSC in terms of potentials, Brown and Ahlers 23] developed a model based on two differential equations which can describe both, azimuthal orientation of the LSC as well as reversals in terms of potentials. These potentials have local minima for the different LSC configurations, thereby the minima are separated by the potential barriers, which can be overcome by turbulent fluctuations. In this picture, the system "jumps" from one state to the other and a reversal of the LSC takes place.

Regarding the dynamics in our system, it seems to be important that the LSCs form a "W"-alignment in a zigzag shape (see Kühn et al. [67|) where warm air rises rather in the rear of the cell and cold air descents in the front. For low Ar, Kühn et al. 67] found no "W"- shape, but rather a wavy FC roll, while for very high $A r$, we observed a bypass flow with the inflowing air descending towards the air outlet directly after entering the cell. In this situation, the LSCs arrange parallel to the short sides of the cell. Thus, only for intermediate $A r$ the above discussed zigzag shape develops. Since oscillations occur only for intermediate $A r$, we conclude that such a zigzag shape is a prerequisite for the generation of temperature oscillations.

An important finding related to the appearance of temperature oscillations is the fixed phase relation between the formation of a new LSC at one and the break down of a LSC at the other side wall. This is another evidence for the coupling of the dynamics at both side walls, which causes the periodicity of the oscillations observed for certain parameter combinations. The existence of such a back coupling is also supported by the rather good agreement of the different examined time scales discussed at the end of Section 6.3.3, which also suggests that the back-coupling process is based on convective transport by the LSCs. The latter, however, occurs only for intermediate $A r$ due to the decorrelation of the LSCs by thermal plumes and background turbulence at high and low $A r$. More precisely, the 
number of plumes per LSC increases with rising $R a$. The latter leads to larger fluctuations and thus, to a decrease in the correlation length. Similarly at low $A r$, that is, rather high $R e$, the turbulent structures generated in the shear layers decorrelate the LSCs. As a result for either too high or too low $A r$, the flow at the side walls decouples and, therefore, no oscillations occur.

To summarise and better envision the dynamics of the convection rolls, we consider rubber straps used in classical mechanics. Each convection roll, neglecting its rotation velocity, is modelled by a rubber strap, and neighbouring rolls are connected to each other. Such a picture is chosen because it underlines the importance of the LSCs as basis for the coupling process and simultaneously includes that the LSCs try to arrange in symmetrical way. A system like this tends to form equally sized rolls by minimising the total force, needed to stretch the straps. This means that a larger roll represented by a highly stretched rubber strap is contracted, while its neighbours expand and vice versa.

The physical motivation of this picture can be described as follows: two neighbouring LSCs tend to have approximately the same rather high temperature $T_{1}$ where the warm air rises. Due to the homogeneous inflow temperature, the rolls must have approximately equal length above the heating plate. There they pick up the same amount of warm plumes rising from the thermal boundary layer, in order to increase the temperature to $T_{1}$. Turbulent fluctuations which are present in the fluid abundantly will move the point where the two rolls touch. Thus, eventually one of the LSCs will have a longer path above the heating plate. However, in the following, the enlarged LSC will shrink again for two reasons: Firstly, the adjacent LSC, which now has a shorter path to travel over the heating plate, and thus gains less buoyancy until it reaches the touching or stagnation point, induces a force to the enlarged roll. This force tends to push the point of rising warm air back. Secondly, the fluid in the enlarged LSC will gather more thermal plumes and thus gains an extra amount of buoyancy which leads to an erstwhile rising and thus a backwards shift of the touching point too. Modelling the LSCs as connected rubber straps takes these motion mechanisms into account.

We first consider three convection rolls, the left one rotating clockwise (see Figure 6.9 left and Figure 6.10, a). In such a structure, a small instability or unbalance between the rising warm air next to the left side wall and the inflowing air can push the rising air away from the wall. Consequently, cold inflowing air can descend next to the left side wall (see Figure 6.10, transition from a to b), which leads to a new convection roll. Thus, following the rubber strap concept which describes the interactions of the rolls, the new roll enlarges while the "old" ones shrink (see Figure 6.10, b). Temporally, this leads to four equally sized rolls, but, as mentioned above, the right roll move towards the side wall where it collapses (see Figure 6.10, transition from b to c). Then, the "new right" roll shrinks as well while the other two rolls grow. Now, the left roll is pulled away from the side wall. Warm air starts to rise next to the side wall and again a new roll is formed on the left side (see Figure 6.10. d). This ongoing process leads to a continuous formation and breakdown of convection rolls and short periods of relaxation of the "rubber straps". In this concept, the influence of $\mathrm{Ar}$ 
on the frequency of the oscillation is interpreted as its influence on the rubber straps spring constant or longitudinal expansion coefficient. However, the exact coupling and interaction of the wall jet with the thermal convection, which are the underlying physical processes, are still an open issue.

Regarding the rubber strap concept, we want to emphasise that the theory might not explain the direction of the movement, but once the movement started, it can explain the ongoing process. A cause for the direction of the movement, in our case always towards the right side wall of the convection cell, might be a tiny spatial imperfection in the inflow velocity distribution, the inlet or outlet height, a spatial imperfection of the heating or the cooling plate, or an other tiny unbalance in the experimental set up, like, for example, a very small tilt angle. Regarding the tilt angle in RBC, Chillà et al. [29] found a very weak, but existing influence on the Nusselt number and Brown and Ahlers 222 observed an effect of the tilt angle on the dynamics of the LSC within a cylindrical RBC sample. That means that even very small tilt angles of the cell might lead to a preferable direction of movement of the convection rolls within our cell.

\subsubsection{LSC MODELS IN RBC}

The next step, after having found such an envisioning picture of the dynamics, is to find a physically motivated model which can describe the observed processes. The development of such a model, which describes the LSC dynamics in MC, however, is still an open issue. Nevertheless, it seems worthwhile already to review the developed models for RBC, to see which ideas or concepts might be adoptable to our case.

Sreenivasan et al. [118] provided a physical picture of the reversal of the mean wind, as a result of their temperature measurements in a cylindrical cell with aspect ratio one. The authors state that the imbalance between buoyancy and friction triggers the reversals, while inertia plays a secondary role. Furthermore, they state that if their simple model holds, various boundary layer instabilities, jets and plumes can provide the reversal mechanism. Fontenele Araujo et al. [39] theoretically analysed the physical mechanism of flow reversals through cessation in turbulent RBC. Both Sreenivasan et al. [118 and Fontenele Araujo et al [39] qualitatively describe the physical mechanisms as follows: for flows with sufficiently strong wind an uprising warm plume might get too fast by a temperature surplus, then it fails to cool down completely while passing the cold top plate. Thus, it is warmer than its surrounding fluid while advected down along the side wall. By buoyancy, it counteracts to the LSC and may stop it or even reverse its direction. Another model for pure RBC in a cylindrical sample with aspect ratio between 0.5 and 3 , which describes a stable LSC with occasional cessations and rotations, in good agreement with experimental results, was proposed by Brown and Ahlers [21] and extended to non-cylindrical geometries [22]. Their model is based on two ordinary differential equations, one for the strength and one for the azimuthal orientation of the LSC. The dynamics of the model is a result of the addition of statistical forces. These forces can be interpreted as the action of the turbulent 
fluctuations that exist throughout the system. As a result the, LSC is stabilised by the angular momentum, while the thermally induced turbulent fluctuations destabilise the LSC. Occasionally, the fluctuations are strong enough to overcome a potential barrier and as a consequence a reversal of the LSC occurs.

It is expectable that similar dynamics as described by the models for RBC, for example, reversals, sloshing, rotating and twisting of the LSC, will occur in MC for the same reasons: angular momentum stabilises the LSCs, while turbulent fluctuations destabilise it. In contrast to pure $\mathrm{RBC}$, in $\mathrm{MC}$ the destabilising turbulent fluctuations can be caused by the interaction of the wall jet with the LSCs, additionally to the thermally induced fluctuations. Furthermore, in a system like ours with multiple LSCs, the LSC-LSC interactions matter possibly in the same way as the LSC-wall interactions. However, the cited physical models might also hold for the occurrence of reversals and oscillations in MC in our cavity, but since the wall jet is superimposed to TC, the relevant processes are, despite the reduced symmetry, most likely more sophisticated and will be subject of further studies.

\subsection{SUMMARY AND CONCLUSION}

We observed and studied oscillations of the large-scale circulation in turbulent mixed convection in a rectangular mixed convection cell using temperature probes and smoke visualisations. The measured temperature values could be assigned to certain arrangements of the LSCs in the cell. Thus, the observed temperature oscillations could be ascribed to specific reconfigurations of the LSCs. A periodic change between a three and a four convection rolls as well as spontaneous reconfigurations and steady states were found.

Periodic oscillations were only detected in an intermediate regime of $A r_{\text {eff }}$, from which we conclude that an equilibrium between inertia and buoyancy forces is an essential ingredient for the development of these oscillations. We infer from this that the oscillations are triggered by the interaction of the forced convective wall jet at the ceiling with the thermally driven LSCs. Hereby, it turned out that the effective Archimedes number based on the temperature difference between heated bottom and inflowing air seem to be a better parameter to predict the occurrence of periodic oscillations. However, a comparable strength of buoyancy and inertia forces is not an adequate criterion, since further $R a$ must not be too high. Otherwise only steady configurations are found. Frequency analysis of the sensor signals reveals a pronounced main frequency and smaller peaks at multiples of it. Further, the frequency scales linearly with $R e$, which is based on the inflow velocity, for those cases where periodic oscillations occur. This reflects that the FC contribution determines the frequency of the oscillations.

The maximal observed main frequency of the oscillations reaches a value of $f=2.0 \times$ $10^{-3} \mathrm{~s}^{-1}$ at $A r=1.8\left(A r_{\text {eff }}=2.1\right)$ and $R a=2.0 \times 10^{8}\left(R e=1.3 \times 10^{4}\right)$. This oscillation frequency is about one order of magnitude smaller as compared to the turnover frequencies of both, wall jet and LSCs.

An important result of the presented study is that the oscillation of the LSCs develops 
due to the formation of a new LSC next to one side wall followed by a break down of a LSC next to the other side wall. Consequently, the LSCs couple the momentum transport at opposing side walls. Further, a back-coupling links LSCs breaking down at one side wall to formation of new LSC at the other side wall. Even more, stable LSCs as well as periodically oscillating LSCs can be destroyed by adjusting the inflow velocity, that is, $R e$.

A concept to describe the mechanisms acting to trigger the periodic oscillations was presented, which pictures the convection rolls as elastic rubber straps known from classical mechanics. How exactly wall jet and thermal convective- induced LSCs couple and how the oscillations are triggered are still open questions, which have to be addressed in further studies. In order to shed more light on the details of the observed processes of, for example, roll formation or the interaction between wall jet and buoyancy- driven convection rolls, simultaneously determined velocity and temperature fields on long time scales are highly desirable. Ongoing work in the fields of time resolved tomographic PIV as well as the combination of PIV and particle image thermography (PIT) based on thermochromic liquid crystals (TLCs) promises new insights into the above discussed coupling processes.

\section{ACKNOWLEDGEMENTS}

The authors are grateful to Sven Lange for his help with the visualisations and to Sebastian Wagner for several constructive discussions. 



\title{
7 Temperature AND VelOCITY Measurements in Convective Air FLOWS 101
}

Citation and credit: Reprinted with permission from Measurements Science and Technology, volume 25, article 035302, 2014, DOI:10.1088/0957-0233/25/3/035302, Copyright 2014, IOP Publishing Ltd

Reference: 101]

Title: "Simultaneous Measurement of Temperature and Velocity Fields in Convective Air Flows"

Authors: Daniel Schmeling, Johannes Bosbach and Claus Wagner

Contributions: I performed all measurements, analysed them and made all figures. I wrote the first draft, whose structure has been preserved.

Note: For the sake of homogeneity of this thesis, the spelling was changed from British English with -ize endings in the journal to British English with -ise endings.

\begin{abstract}
Thermal convective air flows are of great relevance in fundamental studies and technical applications such as heat exchangers or indoor ventilation. Since these kinds of flow are driven by temperature gradients, simultaneous measurements of instantaneous velocity and temperature fields are highly desirable. A possible solution is the combination of Particle Image Velocimetry (PIV) and Particle Image Thermography (PIT) using thermochromic liquid crystals (TLCs) as tracer particles. While combined PIV and PIT is already state of the art for measurements in liquids, this is not yet the case for gas flows. In the presented study we address the adaptation of the measuring technique to gaseous fluids with respect to the generation of the tracer particles, the particle illumination, and the image filtering
\end{abstract}


process. Results of the simultaneous PIV/PIT stemming from application to a fluid system with continuous air exchange are presented. The measurements were conducted in a cuboidal convection sample with air in- and outlet at a Rayleigh number $R a \approx 9.0 \times 10^{7}$. They prove the feasibility of the method by providing absolute and relative temperature accuracies of $\sigma_{T}=0.19 \mathrm{~K}$ and $\sigma_{\Delta T}=0.06 \mathrm{~K}$, respectively. Further open issues, that have to be addressed in order to mature the technique are identified.

\subsection{INTRODUCTION}

Thermal convective flows occur in technical applications such as heat exchangers [7] or indoor climatisation 69 and are of great interest in fundamental studies too. Since these flows are thermally driven, simultaneous measurements of the instantaneous velocity and temperature fields are highly desirable, e.g., to study the dynamics of thermal plumes and their influence on the local and global heat transfer in thermal and mixed convection, or to allow for profound statements to issues like passenger comfort and heat transport processes in aircraft cabins 13, 16. Possible measurement techniques in liquids, e.g. water, are the Molecular Tagging Velocimetry and Thermometry (MTV\&T) 55,61] or the combination of Laser Induced Fluorescence (LIF) and Particle Image Velocimetry (PIV) 115]. Another established technique in liquids is the combination of Particle Image Thermography (PIT) and PIV with thermochromic liquid crystals (TLCs) as tracer particles [9, 40, 54, 153. Thereby, this combined technique is based on the well known PIV algorithms for velocimetry 84 and the special behaviour of the TLCs in reflecting light with different wavelengths when having different temperatures for the thermometry [33,114]. Details about the measurement technique can be found in the review papers of Smith et al. [114] and Dabiri [33] as well as in Tropea et al. 131] (see chapter 7.1). However, the above cited references address combined velocity and temperature measurements in liquids only. Ahlers et al. [3] and Puthenveettil et al. 83 showed that flow properties such as large-scale structures, the Nusselt number, i.e. the heat transport, and the length of near-wall plumes, depend on the Prandtl number. Since air flows with a Prandtl number of $\operatorname{Pr} \approx 0.7$ are found not only in many technical applications, e.g. indoor climatisation [69], but also in more fundamental thermal convection problems, the development of a combined velocity and temperature measurement technique for gaseous, especially air flows is very desirable. Thus, the intention of our work is the combination of PIT and PIV using TLCs as tracer particles in air flows. Especially, we are interested in systems with continuous fluid exchange, such as the climatisation problems mentioned above, which poses some additional challenges to the measurement technique.

The feasibility of combined PIT and PIV for pure thermal convection has already been demonstrated in a cubical Rayleigh-Bénard cell with air as the working fluid [31,103]. In these measurements the TLC tracer particles were sprayed into the cubical cell which was closed after the particle injection. After a short settling time, the images for PIT and PIV were recorded with two cameras, a grey level double shutter camera (Pixelfly QE, (C) PCO) and a commercial digital reflex camera (D70s, C)NIKON). This setup allowed first 
measurements in thermally driven air flows in closed systems with no air exchange.

Other possibilities for acquiring the temperature and the velocity field in air flows are the combination of LIF and PIV [115] or the acoustic tomographic imaging of the temperature and flow field $[6]$. Nevertheless, these promising techniques are either just proposed and not yet accomplished (LIF/PIV), or reveal only a very poor resolution so far (acoustic tomographic imaging).

Furthermore, Fujisawa and Hashizume [41] have suggested that highly accurate measurements of the velocity can only be carried out using additional tracer particles in order to satisfy the condition for PIV analysis. Whether and to what extent this claim holds for the adaptation of this combined measurement technique to air flows will be analysed in the near future by a comparison of conventional PIV results with those acquired with white light and TLC tracer particles.

The present paper is intended as a step towards simultaneous measurements of instantaneous temperature and velocity fields in air flows in open systems with air exchange using TLCs. It is structured as follows: Section 7.2 first reviews some of the fundamentals of TLCs, then discusses the new achievements in particle generation, their illumination, and finally the issues of the image filtering process. This section is followed by a paragraph on the application of the novel measurement technique to a convection experiment. A summary and conclusion including an outlook is given at the end of the article.

\subsection{General Considerations of Combined PIT and PIV}

The general requirements which must be fulfilled to apply TLCs as tracer particles in air flows for combined PIT and PIV are the following: First, in order to conduct PIT, they have to provide a temperature-dependent reflection of different wavelengths with a short temperature response time. Second, for accurate PIV, the tracer particles must possess good following behaviour, a high light scattering efficiency and a long lifetime. Additionally, for systems with continuous fluid exchange, the tracer particles need to be continuously produced at a high rate. Furthermore, it has to be considered that the colour play of the TLCs, which is exploited in PIT to locally detect the fluid temperatures, not only depends on the temperature but also on, e.g. the angle between the incident illumination and line of view, the background light, as well as the size and the age of the droplets. Consequently, a spatially resolved calibration is needed for high precision measurements, and TLC particles with a narrow size distribution have to be generated. While all of the above discussed issues are addressed in our ongoing study, the present paper focuses on recent progress in particle illumination and characterisation as well as image processing.

\subsubsection{Fundamentals of Thermochromic Liquid Crystals}

Based on the review papers of Smith et al. 114$]$ and Dabiri [33], some of the fundamentals of TLCs, especially some of the colour-dependent parameters, are described in the following. 
It is well known that the colour of a TLC droplet depends not only on its temperature but also on, e.g. the angle between the incident illumination and line of view, the background light and the age of the droplet. Due to the angular dependence, a local calibration provides a far better accuracy than a global calibration. Another important parameter is the size of the droplets. Hence, a major requirement is that the produced particles have a sharp size distribution. In this respect the minimal/maximal possible size of the TLCs, which depends on two factors, must be detected. On the one hand the TLCs must maintain their temperature-dependent reflection of different wavelengths (the play of colours), i.e. they must be as large as possible, and on the other hand, they need the best possible following characteristics, i.e. they must be as small as possible.

From previous studies by Czapp [31] and investigations of the colour play of the crystal as function of the particle generator settings, we know that the diameter of the TLCs should be of the order of $10 \mu \mathrm{m}$. An estimate of the reaction time of the TLCs to temperature changes, the following behaviour and the sinking velocity is given in Czapp 31 and Raffel et al. 84. There, the reaction time based on the heat transport from the fluid to crystal via convection $\left(\tau_{\alpha}\right)$ is estimated as $\alpha=N u \cdot \lambda_{t, \text { air }} \cdot d_{\mathrm{p}}^{-1}$ using $N u=2$ for very creeping flows. Here, $\alpha$ is the heat transmission coefficient. The other possible heat transfer mechanism from the fluid to the crystal is heat radiation $\left(\tau_{\sigma}\right)$, which can be approximated using the Stefan-Boltzmann law and the emissivity $\epsilon=1$. Once the heat is transferred from the fluid to the crystal, or vice versa, the surrounding is colder than the crystal. Thus, the crystal itself must transport the heat to its interior by heat conduction $\left(\tau_{\lambda}\right)$, which can be estimated based on the heat conductivity of the crystal $\lambda_{\mathrm{p}}$. Finally, the helical structure of the crystal, i.e. the colour of the crystal, must change according to the temperature of the crystal. Since this relaxation time $\tau_{\text {relax }}$ is hard to estimate we refer to the value $100 \mathrm{~ms}$ for cholesteryl ester and $1-10 \mathrm{~ms}$ for chiral nematic crystals published in [12,56, 57, 143]. Thus, based on the above discussed assumptions, one obtains the following estimates for the relevant reaction times for the colour change of a TLC particle with a diameter $d_{\mathrm{p}}=10 \mu \mathrm{m}$ :

$$
\begin{aligned}
\tau_{\alpha} & \approx \frac{C_{p} \cdot d_{\mathrm{p}}}{6 \cdot \alpha} \approx \frac{C_{p} \cdot d_{\mathrm{p}}^{2}}{12 \cdot \lambda_{t, \text { air }}}=1.0 \mathrm{~ms}, \\
\tau_{\sigma} & \approx \frac{C_{p} \cdot d_{\mathrm{p}}}{24 \cdot \sigma \cdot T^{3}}=0.8 \mathrm{~s}, \\
\tau_{\lambda} & \approx \frac{C_{p} \cdot d_{\mathrm{p}}^{2}}{4 \cdot \lambda_{\mathrm{p}}}=0.3 \ldots 2.5 \mathrm{~ms}, \\
\tau_{\text {relax }} & =1 \ldots 10 \mathrm{~ms},(100 \mathrm{~ms} \text { for cholesteryl ester crystals }),
\end{aligned}
$$

with the heat capacity per volume of $C_{p}$, the Stefan-Boltzmann constant $\sigma$, and the ambient temperature $T$. The above shown three orders of magnitude higher reaction times of the radiative heat transport to the particle $\left(\tau_{\sigma}\right)$ is negligible compared to the heat transport via convection $\left(\tau_{\alpha}\right)$. Contrarily, the time constant for the internal heat conduction within the particle $\left(\tau_{\lambda}\right)$ is like that of $\tau_{\alpha}$ of the order of milliseconds as well. Another important time constant is the time that the TLC needs to change its colour after having changed its temperature $\tau_{\text {relax }}$. For thin layers of chiral nematic crystals, which is the crystal type 
we use in our experiments, values in the range from $1-10 \mathrm{~ms}$ are reported for $\tau_{\text {relax }}$ in the literature [12,56,57, 143]. Although the lateral dimensions of the TLCs might affect $\tau_{\text {relax }}$, the values for small droplets of chiral nematic crystals are similar. Dabiri [33, report response times for TLC particles from Hallcrest with a diameter of $10 \mu \mathrm{m}$ of $1-4 \mathrm{~ms}$. Tropea et al. [131] give a response time of $3-10 \mathrm{~ms}$ for TLC tracer particles with diameters of $20-50 \mu \mathrm{m}$. However, much larger values of up to 100 milliseconds are reported for another crystal type, i.e. cholesteryl ester (cholesteric) crystals $12,56,57$. . According to the discussion above we expect the overall thermal response time $\tau_{\mathrm{t}}$ to be below $10 \mathrm{~ms}$ for our chiral nematic crystals (Hallcrest, R20C6W) with a mean diameter of approx $10 \mu \mathrm{m}$. It is a function of the processes discussed above, some of which proceed in parallel while others take place consecutively. However, our analysis revealed the relaxation time as limiting time scale, while direct measurements of $\tau_{\text {relax }}$ of dispersed TLC particles in air have not been reported so far and constitute an open issue. Though, in spite of the fast response time compared to conventional temperature sensors, precise temperature measurements are possible only if the TLC particles are able to adapt their temperature on time scales comparable to that of the local convective velocity and the spatial resolution of the measurement. To quantify this, we estimate the maximal travelled distance $\delta_{\min }=\tau_{\mathrm{t}} \cdot u_{\max }$ during the reaction time $\tau_{\mathrm{t}}$ which ought to be smaller than the minimum of the interrogation window size and the size of the smallest coherent structures, i.e. approximately ten times the Kolmogorov length $\eta_{\mathrm{K}} 123$ :

$$
\begin{aligned}
& \delta_{\min }<d_{\text {int. window }} \\
& \delta_{\min }<10 \cdot \eta_{\mathrm{K}} .
\end{aligned}
$$

In our measurements the maximal fluid velocity is $u_{\max } \approx 0.1 \mathrm{~m} / \mathrm{s}$, thus $\delta_{\min } \approx 10^{-3} \mathrm{~m}$, while the $10 \cdot \eta_{\mathrm{K}} \approx 10^{-2} \mathrm{~m}$ (for $R a \approx 10^{8}$ and $\operatorname{Pr} \approx 0.7$ ) [123] and the interrogation window size amounts to $d_{\text {int. window }} \approx 5 \cdot 10^{-3} \mathrm{~m}$. Hence, the thermal reaction time is short enough for the here considered flows.

An overview of the parameter range, in which the technique is applicable according to the above discussed limitations is depicted in figure 7.1. The dark grey shaded triangle in figure 7.1, in which the measurement technique can be applied, reflects that the spatial resolution not only decreases with increasing velocity but also crucially depends on the reaction time, which lies between one and $10 \mathrm{~ms}$. The region of resolvable spatial fluctuations depending on the maximal fluid velocity $u$ is highlighted in light and dark grey for $\tau_{\mathrm{t}}=1 \mathrm{~ms}$ and $\tau_{\mathrm{t}}=10 \mathrm{~ms}$, respectively. For the interrogation size of the measurements presented below, which is marked as the black dot in figure 7.1, the response time of $\tau_{\mathrm{t}}=10 \mathrm{~ms}$ is small enough. Though, it must be noted that if this is not the case backtracing of the particle positions using the measured velocity field with sophisticated tracing algorithms allows to enlarge the parameter range in Fig. 1 in which the measurement technique can be applied.

Other important parameters, which might limit the accuracy, are the mechanical reaction time $\tau_{\mathrm{m}}$ and the sinking velocity due to gravity $v_{\mathrm{g}}$. They can be estimated (following Raffel 


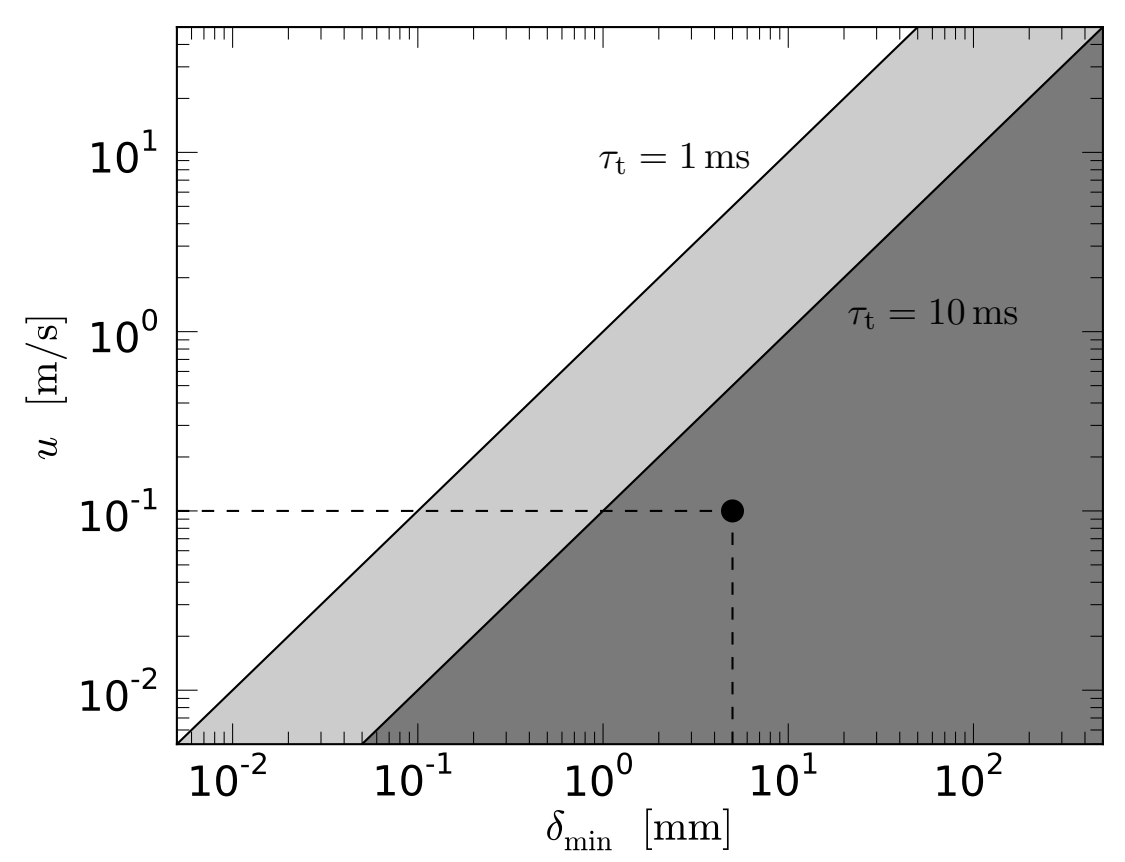

Figure 7.1: Parameter range dependent on the spatial resolution depending on the thermal reaction time of the TLCs and the maximal fluid velocity with applicability range of the measurement technique shaded in dark grey. The values of our measurement are marked with the black dot.

et al. [84]) assuming Stokes friction:

$$
\begin{aligned}
v_{\mathrm{g}} & =d_{\mathrm{p}}^{2} \cdot \frac{\rho_{\mathrm{p}}-\rho}{18 \cdot \eta} \cdot g \approx 3 \mathrm{~mm} / \mathrm{s} \\
\tau_{\mathrm{m}} & =d_{\mathrm{p}}^{2} \cdot \frac{\rho_{\mathrm{p}}-\rho}{18 \cdot \eta} \approx 0.3 \mathrm{~ms}
\end{aligned}
$$

using the particle density $\rho_{\mathrm{p}} \approx 1.01 \cdot 10^{3} \mathrm{~kg} / \mathrm{m}^{3}\left(\rho_{\mathrm{TLC}}=1.00-1.02 \cdot 10^{3} \mathrm{~kg} / \mathrm{m}^{3}\right.$ given by Hallcrest for the unencapsulated TLCs used by us), the gravitational acceleration $g$ as well as the density $\rho \approx 1.2 \mathrm{~kg} / \mathrm{m}^{3}$ and the dynamic viscosity $\eta \approx 1.8 \cdot 10^{-5} \mathrm{~kg} /(\mathrm{m} \cdot \mathrm{s})$ of air at $20^{\circ} \mathrm{C}$. Although this sinking velocity is lowered by the Brownian motion, it is still a limiting factor for the total measurement time without supplying new particles and the accuracy of the velocity measurement. Again based on the assumption of Stokes, Raffel et al. 84 describe the following behaviour of the smallest particles while travelling through a shock wave:

$$
v_{\mathrm{p}}(t)=v_{\infty}\left[1-\exp \left(-\frac{t}{\tau_{\mathrm{m}}}\right)\right]
$$

with the velocity of the particle $v_{\mathrm{p}}(t)$ and the velocity of the surrounding fluid $v_{\infty}$. These estimates result for a $d_{\mathrm{p}}=10 \mu \mathrm{m}$ TLC particle in an air flow in a time of $t \leq 3 \mathrm{~ms}$ that the particle needs to reach $99 \%$ of the fluid velocity. Thus, TLC particles with $d_{\mathrm{p}} \leq 10 \mu \mathrm{m}$ follow rather slow fluid flows almost instantaneously.

To calculate the temperature from the colour of the TLC particles, a transformation of the colour space must be applied. For the TLC particles used with this specific diameter, 
a calibration of the temperature-dependent hue value [33] has to be performed, so that a temperature accuracy better than $\pm 0.3 \mathrm{~K}$ is achieved. Thereby the hue is, besides saturation and value or saturation and lightness, one of the three parameters of the HSV or HLS colour space, respectively. For graphical representations of the colour spaces, see figure 7.2. The parameter value is the only non-obvious parameter, and can be understood as the brightness (or darkness) value of the colour with $V=0$ being black and $V=1$ being the pure colour. The hue saturation intensity (HSI) colour space is another possible colour space which can be used for the calibration of temperatures to colours. Its difference from the HLS colour space is only the third parameter: lightness is the relative light intensity and intensity is the absolute one. Using either one of these doesn't matter much. For completeness it should be noted that the hue saturation brightness (HSB) colour space, which some of the literature refers to, is exactly the same as the HSV colour space, only naming the parameter more obviously brightness instead of value. Nevertheless, in the following, we will use the HSV name instead of HSB because it is the more common expression.

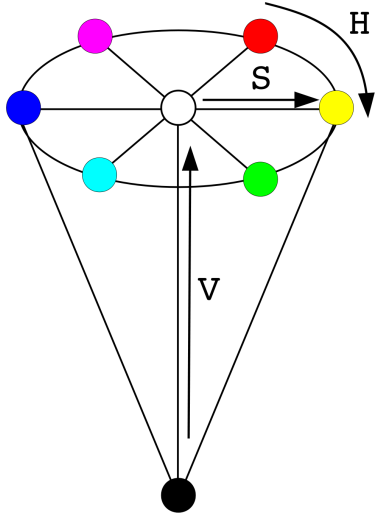

(a)

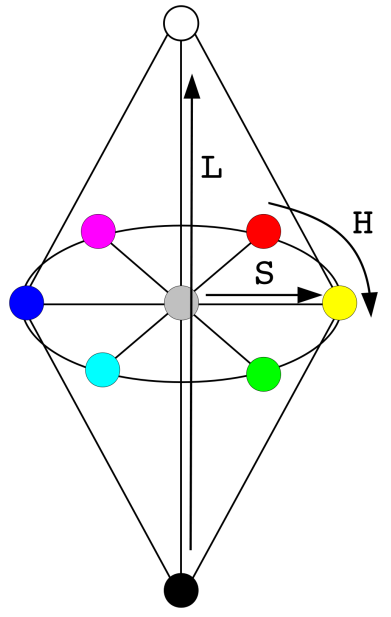

(b)

Figure 7.2: Representations of (a) the HSV and (b) the HLS colour space.

The hue value $h$ can be calculated from the RGB values (red: $r$, green: $g$, blue: $b$ ) 44 by:

$$
h(r, g, b)= \begin{cases}\frac{g-b}{\max (r, g, b)-\min (r, g, b)}, & \text { if } r=\max (r, g, b) \\ \frac{b-r}{\max (r, g, b)-\min (r, g, b)}+2, & \text { if } g=\max (r, g, b) \\ \frac{r-g}{\max (r, g, b)-\min (r, g, b)}+4, & \text { if } b=\max (r, g, b)\end{cases}
$$

The parameters saturation and lightness, intensity or value can be used to correct artifacts like black or white pixels of the image and for the filtering process which is needed for the separation of the particles from the background. A possible realisation of this process is described in section 7.2.4. All colour information needed to calculate the temperature is expressed with the single parameter hue, hence this parameter is used to calculate the temperature from the colour image. 


\subsubsection{Generation of Tracer Particles}

For the production of the tiny unencapsulated TLC particles, a generator is needed that fulfils the following requirements: Firstly, to investigate systems with air exchange, a continuous production of the particles must be realised. Secondly, the particles have to exhibit a sharp size distribution in order to allow for a well defined colour play and finally, the mean particle size, however, should be adjustable by the operational parameters of the generator in order to allow matching of the size-dependent particle colour play to the actual measurement. The final particles must, on the one hand, maintain their temperaturedependent reflection of different wavelengths (the play of colours), i.e. they must be as large as possible. On the other hand, they need the best possible following characteristics, i.e. they must be as small as possible.

While in previous studies, particle generation based on the Rayleigh instability [86], which potentially provides some of the above mentioned requirements, was employed [103], two different generators have been tested. One is based on an air-atomising nozzle $(0.25 \mathrm{~mm}$ nozzle diameter, liquid nozzle: PF1050, air nozzle: PA64, (CSpraying Systems) and the other one is an airbrush system $(0.3 \mathrm{~mm}$ nozzle diameter). A inverted grey scale image of the particle jet produced by the airbrush system is presented in figure 7.3 . Both generators provide a particle production rate that is sufficient for measurements on the square meter scale. To generate the tracer particles, the TLC mixture is first dissolved in isopropyl alcohol. In the second step, the mixture is atomised by the generator. After evaporation of the isopropyl alcohol from the atomised droplets, atomised TLC tracers remain. However, the resulting particles have no monodisperse size distribution, see figure 7.4(a) and (b). Due to the above mentioned aspects, it is almost impossible to measure the TLC particle size directly. Therefore we used water instead of the TLC mixture and measured the particle size distribution produced with the generators by applying Phase Doppler Anemometry (PDA). The diameter statistics of the generated water droplets for both generators at their working points are presented in figure 7.4. All droplet sizes were measured with a PDA system assuming spherical droplets. The particle generators used provide a sufficient number of particles with the desired density and size distribution and are therefore well suited for simultaneous PIT/PIV investigations. A further analysis of the particle diameter distribution of the generators revealed that the airbrush system provides droplets whose diameters follow a log-normal distribution, see the Gaussian curve on a logarithmic $x$-axis (inlay of figure 7.4, a). For the air-atomising nozzle, no such simple particle distribution was obtained. The semilogarithmic plot (inlay of figure 7.4 b) reveals that there is a rather high proportion of large particles. These particle statistics underline that the airbrush system provides a sharper size distribution and, therefore, appears to be more suitable for our purposes. Nevertheless, more development work can be performed with respect to the generation of TLC particles, to further optimise, e.g. the dispersity of the generated particles.

In our experiments, the mass mixing ratio between the TLC mixture and isopropyl 
alcohol was set to $1: 2$ or $1: 1$, eventually resulting in a TLC particle size smaller than the produced droplets by a factor of approximately 1.4 or 1.3 in diameter, respectively. Of course, variation of the mixing ratio opens up another possibility to vary the TLC tracer particle size.

For the results presented in this paper, we used the airbrush system and a mixing ratio of $1: 1$ for the particle generation. The size of most of the produced TLC-isopropyl alcohol droplet turned out to be approximately $10 \mu \mathrm{m}$, see figure 7.4 (a). Thus, the TLC particle diameter should be in the range of $d_{\mathrm{p}} \approx 7 \mu \mathrm{m}$. The particles produced with this combination of generator and mixing ratio, led so far to the most convincing results in simultaneous PIV and PIT. Please note that according to the assumptions given by Raffel et al. [84], discussed above, the mechanical reaction time until the particles reach $99 \%$ of the fluid velocity is in the order of $\tau_{\mathrm{m}} \approx 1.5 \mathrm{~ms}$. For the rather slow flows considered here, the particles are assumed to follow the surrounding fluid instantaneously.

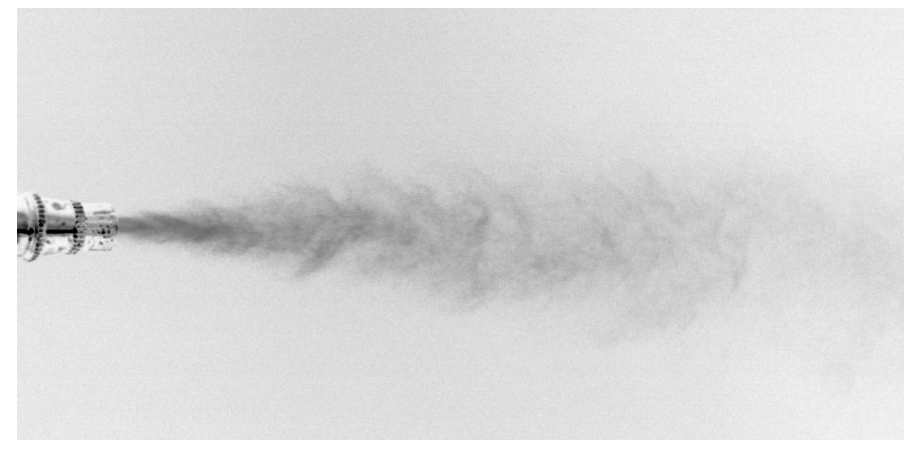

Figure 7.3: Inverted greyscale image of the particle jet produced by the airbrush system.

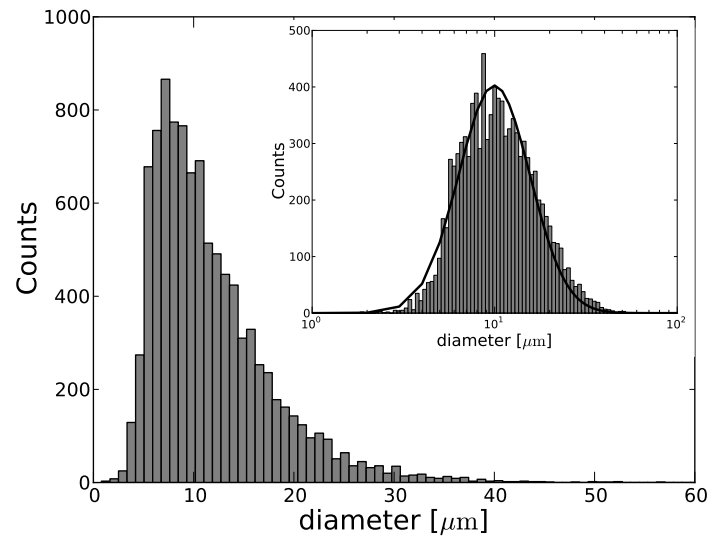

(a)

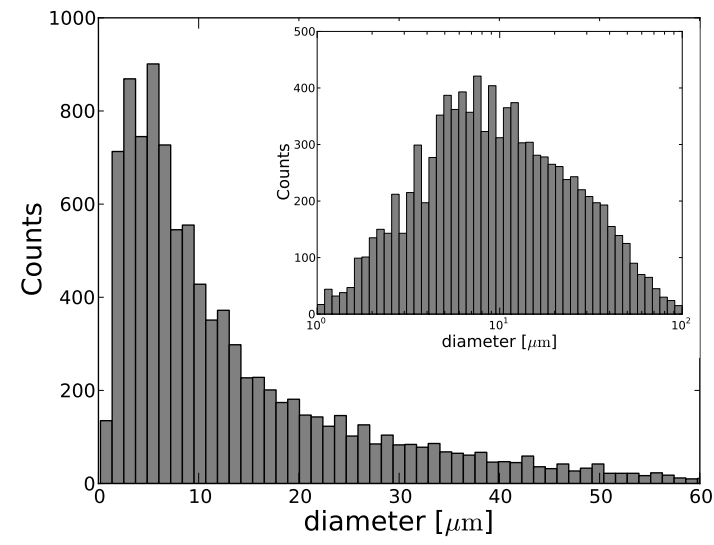

(b)

FiguRE 7.4: Diameter statistics of generated water droplets for both particle generators: (a) airbrush system, (b) air-atomising nozzle at $p_{\text {fluid }}=0.2$ bar and $p_{\text {air }}=0.3$ bar, inlays: logarithmic $x$-axes. 


\subsubsection{White Light Sheet}

One of the main components in a simultaneous PIT/PIV setup is the white light sheet. For high quality PIV measurements, it should provide an intensity that is high enough and a collimation that is good enough to allow sharp imaging of single tracer particles. This implies also that the pulses of the light source must be short enough to not smear the particle images. Furthermore, the flashing frequency should be as high as possible, to achieve the best possible time resolution as well.

The white light sheet employed in our measurements is based on Light Emitting Diodes (LEDs) and was specially developed for this purpose. The LEDs have the advantages of low cost, easy handling, and good luminous efficiency. However, their drawbacks are the low coherence and high divergence of the emitted light and their finite size, i.e. they can not be considered as point sources. A successful implementation of LEDs for PIV investigations was already reported in Willert et al. [145. Due to the need for a high light intensity, white colour and a good focusability at the same time in our application, we developed a light sheet source based on 60 COSRAM Platinum Dragon LEDs, LW W5SN-KYLY-JKQL, which are arranged in a one-dimensional array on a printed circuit board. Each single LED is equipped with a clip-on lens (C)SHOWIN Clip-On Lens for Osram GD + LED) in order to reduce the angle of radiation from approximately $120^{\circ}$ to approximately $15^{\circ}$. Another main advantage of the applied clip-on optics is their small spatial extension, which is just a bit larger than the LEDs are, allowing the achieving of a spatial density of 11.6 LEDs per $10 \mathrm{~cm}$. The one-dimensional array of LEDs is used in combination with a slit aperture and a cylindrical lens. By adjusting the distances between the different elements, one can manipulate the brightness and divergence of the produced light sheet. A sketch of the light sheet optics is shown in figure 7.5 .

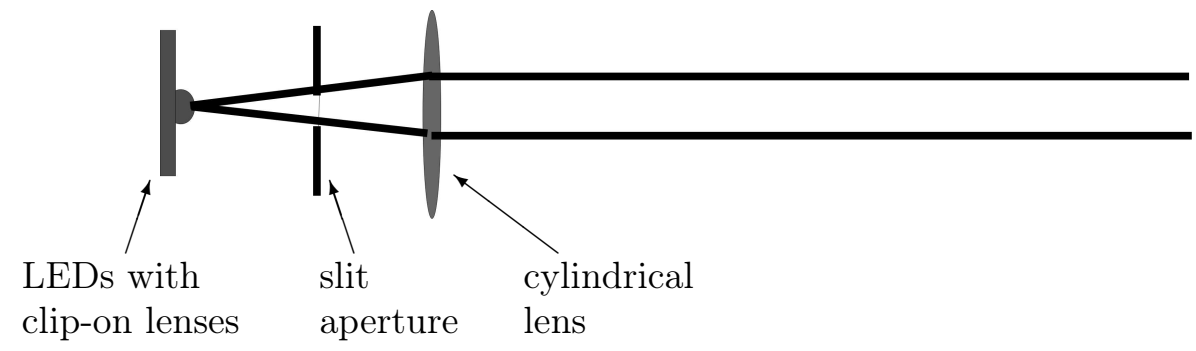

FIGURE 7.5: Sketch of the new developed white light sheet optics, including the LED array, the clip-on optics and a combination of a slit aperture and a cylindrical lens for the formation of the light sheet.

Each of the white light emitting LEDs has a luminous flux of $191 \mathrm{~lm}$ at a forward current of $1 \mathrm{~A}$ 78. PIV investigations require a pulsed light source, this implies that the LEDs can be operated at an increased forward current of 3.3 A. For reasonable PIV results in thermal convection at $\operatorname{Pr} \approx 0.7$ and moderate $R a$ numbers, a pulse length equal to or shorter than approximately $1 \mathrm{~ms}$ is sufficiently short. The duration of the light pulses and the time delay between the first and the second pulse for PIV can be controlled with an external trigger 
unit using TTL signals. The time response of the light source to a double pulse TTL trigger signal was measured using a photodiode, placed in the light sheet, and a oscilloscope. For the characterisation of the light source only, the delay between the two pulses was set to $\tau=5 \mathrm{~ms}$ and the duration of each pulse to $1 \mathrm{~ms}$. While the pulse duration is kept at $1 \mathrm{~ms}$ during our actual experimental investigations, the delay time is adjusted to $\tau=16 \mathrm{~ms}$ in order to get a maximal pixel displacement in the order of $\Delta x \approx 10 \mathrm{px}$. Figure 7.6 shows the time traces of the light sheet output and a the trigger pulse. Thereby, figure 7.6 (a) depicts both pulses, necessary for PIV investigations, and (b) sketches a magnification of just one pulse, to show the reaction of the light output on a trigger signal. The rise and fall times of the light output are $44 \mu \mathrm{s}$ and $63 \mu \mathrm{s}$, respectively.

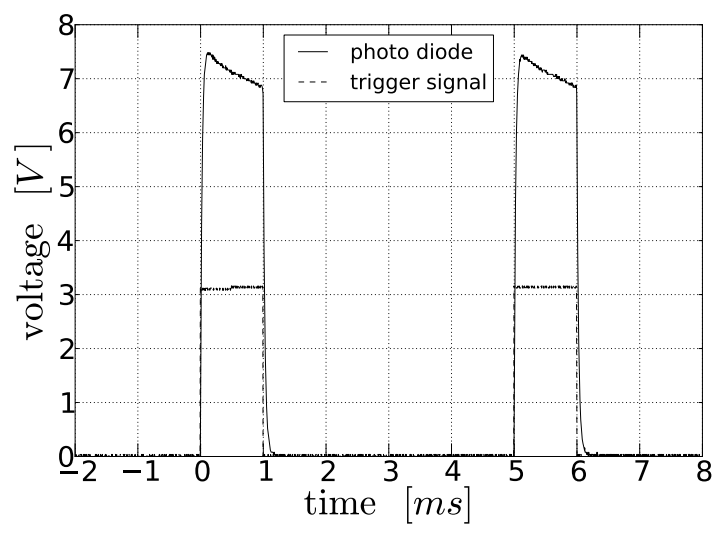

(a)

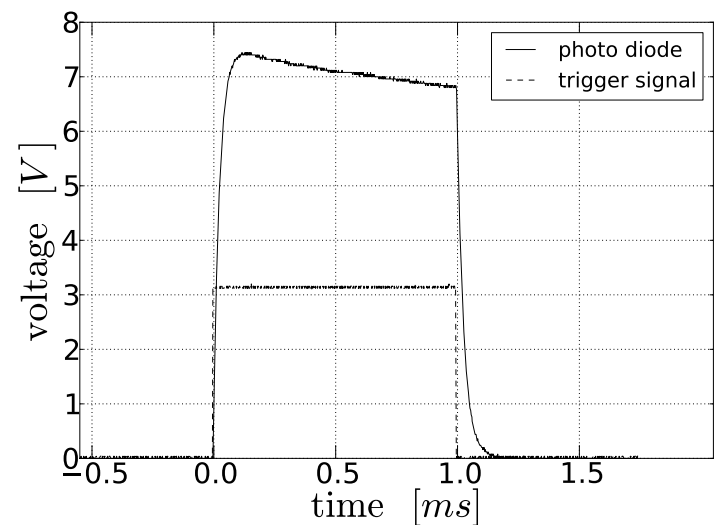

(b)

Figure 7.6: Time trace of light emission as detected by a photodiode $(-)$ as response to a TTL trigger signal (--): (a) $1 \mathrm{~ms}$ light pulse duration and a time delay between the first and the second trigger signal of $5 \mathrm{~ms}$, (b) magnification of one of the two pulses depicted in (a).

The recorded pulse duration of $1 \mathrm{~ms}$ is sufficiently short for investigation of rather slow flows, e.g. indoor climatisation, but may not be applicable for high flow velocities (also depending on the used camera and lens system). At high flow velocities, such a long pulse duration will lead to elliptically stretched rather than sharp circular images of the tracer particles, and therefore will lead to a decreasing accuracy of the velocity evaluation. We also tested a shorter light pulse length of $0.5 \mathrm{~ms}$, which makes it possible to measure faster flows as well. Due to a better signal to noise ratio and no drawbacks in the quality of the particle images, we used a pulse length of $1 \mathrm{~ms}$ for the study presented here. It should be noted that $\tau=5 \mathrm{~ms}$ was fixed for the characterisation of the light source, only. During the measurements, $\tau$ was adjusted to get pixel displacement values which are suitable for the PIV interrogation, see section 7.3 .2 .

Figure 7.7(a) presents intensity profiles of the lightsheet which were measured using a photodiode mounted onto a traverse stage. For all presented distances $(100 \mathrm{~mm} \leq x \leq$ $500 \mathrm{~mm}$ ) from the front lens of the light sheet optics, a Gaussian profile was found. To 
further characterise the light sheet, the maximal values of the photo voltage as well as the full width of the profiles at half maximum (fwhm) are presented in figure 7.7(b) for distances from $50 \mathrm{~mm}$ to $1000 \mathrm{~mm}$. One finds that for $x \leq 500 \mathrm{~mm}$ the maximal photo voltage (i.e. the luminous intensity of the light sheet) drops only by $25 \%$, while the thickness in terms of fwhm is always less than $10 \mathrm{~mm}$. For larger distances, the maximal photo voltage drops rather quickly, to approximately $30 \%$ of its maximum, and the light sheet thickness increases up to $18 \mathrm{~mm}$ at $x=1000 \mathrm{~mm}$.

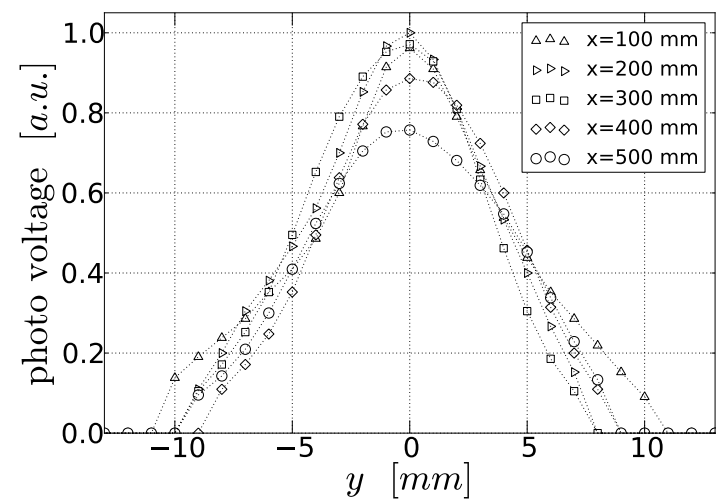

(a)

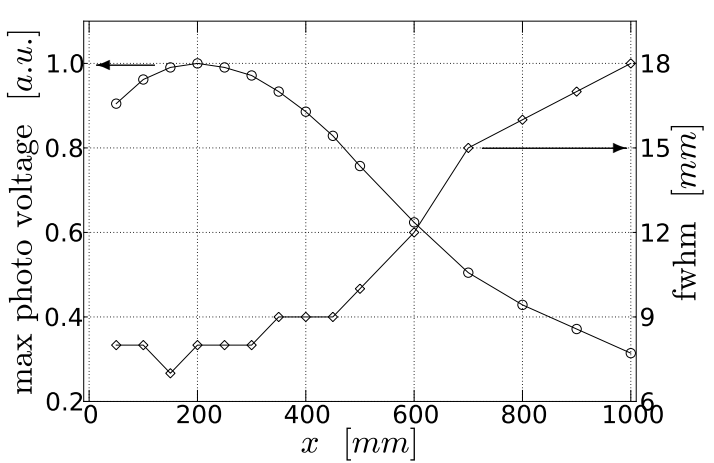

(b)

Figure 7.7: (a) Intensity profiles of the lightsheet (at half height of the lightsheet) and (b) maximal intensity and full width at half maximum (fwhm) for various distances form the front lens, measured using a photodiode.

Thus, summarising, we can state that the developed white light sheet optics produces a light sheet with a thickness (fwhm) of approximately $9 \mathrm{~mm}$, very low divergence, and rather homogeneous illumination over a distance of $500 \mathrm{~mm}$.

The repetition rate is, at present, set to $4 \mathrm{~Hz}$ and in our investigations limited by the cameras used rather than by the light sheet optics. It is much higher than the repetition rate of approximately $1 / 5 \mathrm{~Hz}$ that Czapp [32 was able to realise. Furthermore, the new frequency of $4 \mathrm{~Hz}$ allows for well time-resolved studies of rather slow processes, e.g. the indoor climatisation or convection driven flows at low and moderate Rayleigh numbers in which we are interested.

\subsubsection{Image Filtering Process}

A characteristic difficulty of PIT of air flows arises from the large difference of index of refraction between fluid and particle. While this is advantageous for parallel PIV measurements, the high amount of Mie-scattered white light tends to overcast the colour information of the inelastically scattered light. Precise PIT measurements thus require special attention during image processing in order to separate the temperature dependent colour information from the background noise. In order to keep the amount of scattered white light to a minimum, the PIT camera was positioned at an angle of approximately 
$45^{\circ}$ in the backward direction, which already reduces the background significantly. Such a camera setup for PIV leads to perspective errors associated with the out of plane velocity component. While the backward position of the colour camera is essential to receive a sufficient image quality for PIT, the viewing angle of the PIV camera can be chosen independently. In the current study the PIV camera has been installed viewing slightly into backward scattering for practical reasons. However, in future measurements the PIV camera can be operated perpendicular to the field of view as well, while the colour camera is maintained in backward direction, as long as the optical accessibility of the setup allows it.

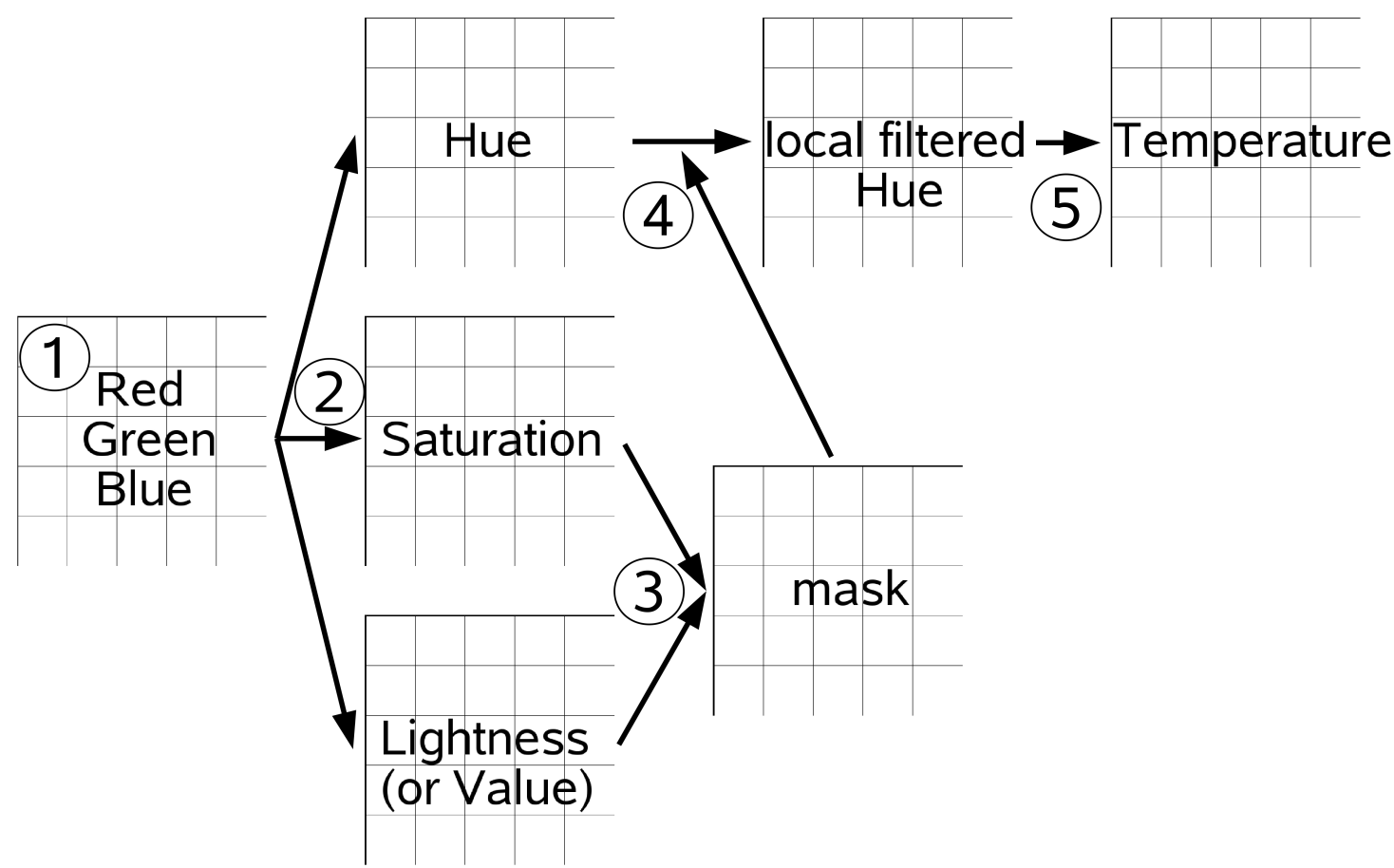

Figure 7.8: Sketch of the image filtering process. Step 1: recorded RGB image, step 2: decomposed HLS / HSV image, step 3: generation of the mask using L and S or S and V, step 4: application of the mask to $\mathrm{H}$, step 5: calculation of the local temperatures using hue-temperature calibration functions.

The images for both the PIV and PIT are recorded simultaneously with two Pixelfly (C)PCO) cameras, one grey level double shutter and one colour camera. The double shutter camera is used for the well known PIV evaluation (see e.g. [84]). For the temperature measurements the colour camera is used. This camera records red/green/blue (RGB) images with a Bayer filter (step 1 in figure 7.8). Both images are deskewed and overlapped using calibration grids and are projecting tools similar to those used for stereoscopic PIV.

Since no neutral sunlight is used for illumination, a specific white balance is fundamental for acquiring realistic and reproducible colours. Therefore, we use a neutral greycard which has a remission of light that is independent of the wavelength of the incident light. Such a card is well known in photography. It allows to determine a reference value for each setup, in particular for a specific arrangement of the illumination, and thus to choose the right 


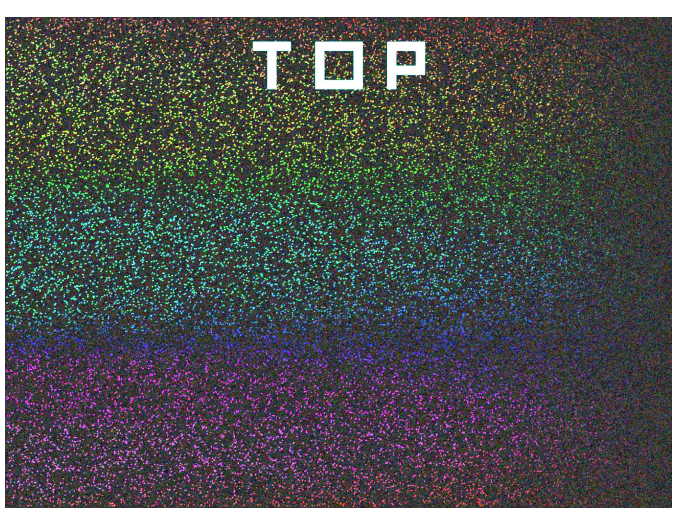

(a)

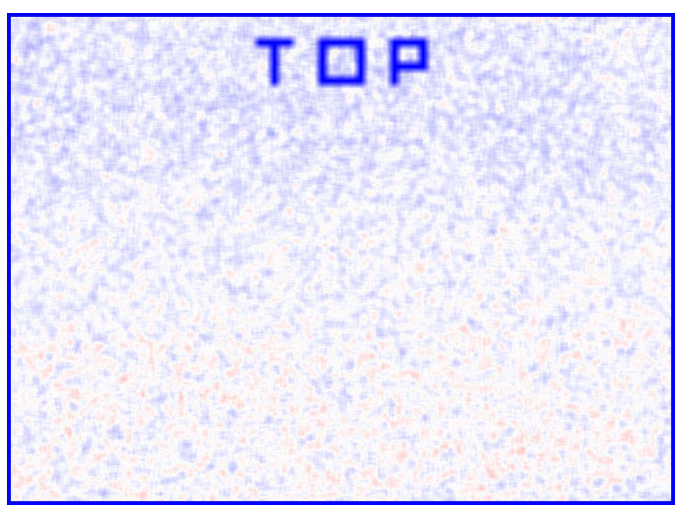

(c)

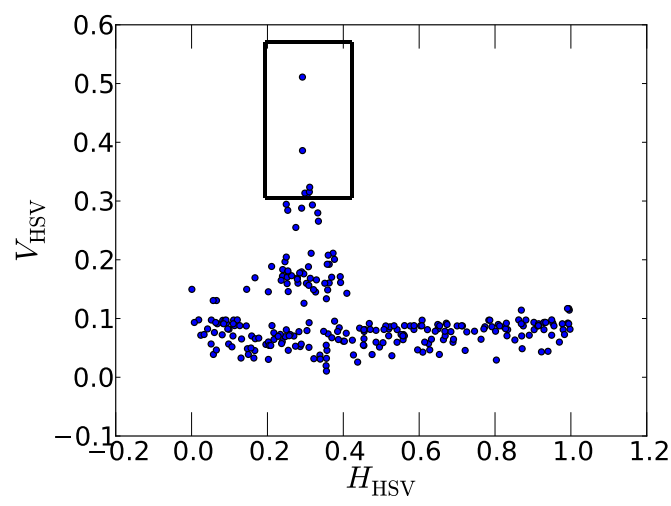

(e)

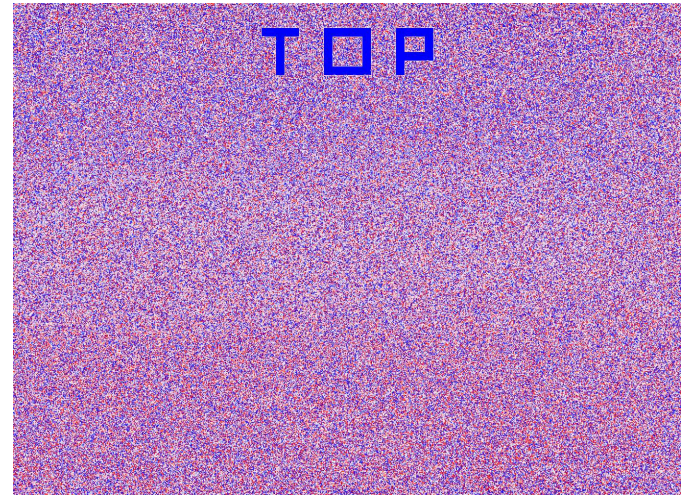

(b)

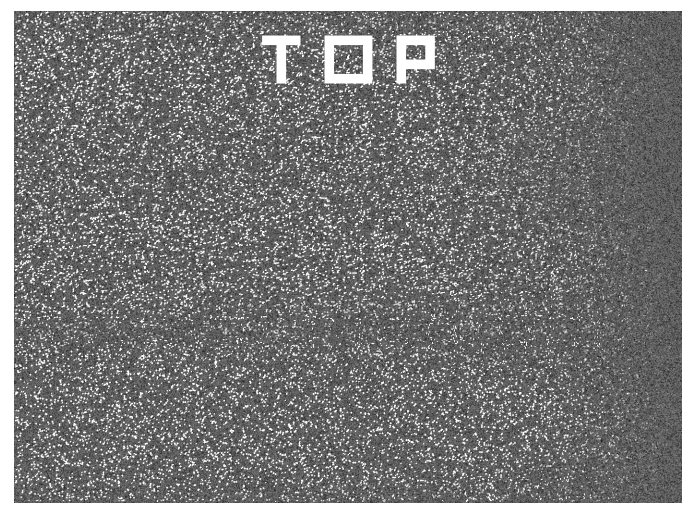

(d)

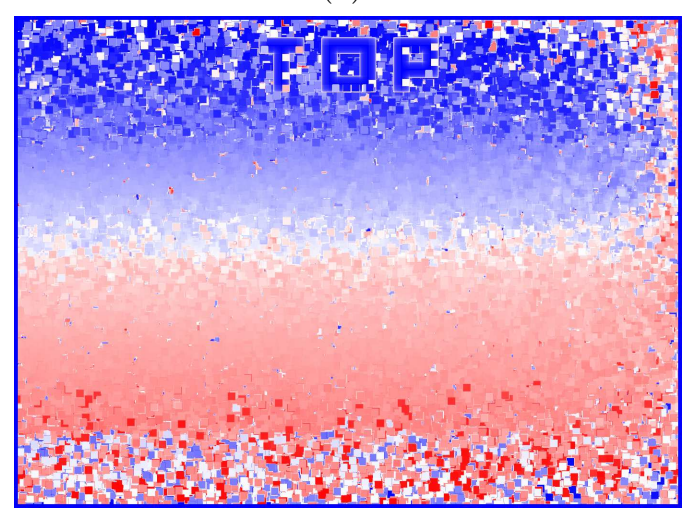

(f)

0

(g)

FIGURE 7.9: Filtering process of the raw images for the separation of the particles from the background noise: (a) synthetic colourful particle image (RGB), (b) hue channel (in HSV colourspace), (c) mean hue value in $16 \times 16 \mathrm{px}$ interrogation windows, (d) value channel (in HSV colourspace), (e) hue vs. value for a $16 \times 16 \mathrm{px}$ interrogation window, box marks those pixels with highest four value levels which are used for the hue calculation within this window, (f) final hue image in $16 \times 16 \mathrm{px}$ interrogation windows after filtering using a threshold in the value channel, (g) colour scale for images (b), (c) and (f). 
white balance which guarantees a neutral colour and their reproducibility.

For filtering and temperature analysis, these images are converted into the hue/saturation/value (HSV) or the hue/lightness/saturation (HLS) colour space (step 2 in figure 7.8). Due to the finite width of the particle size distribution, the recorded images reveal a rather high amount of background noise. In order to take only the colourful particles into account, a threshold for the value (HSV) is used in order to filter the hue values (step 3 and 4). Different filter algorithms could also be based on the saturation (HSV or HLS) or the lightness (HLS). Finally, the local temperatures can be calculated using hue-temperature calibration functions (step 5).

The main challenge is to identify those pixels which are neither background noise, nor small particles scattering white light. This can be done in different colour spaces. For a realisation in the HLS colour space, only those pixels should be taken into account that have a high saturation and a lightness in the range of $\epsilon<L<1-\epsilon$ with $\epsilon>0$. In the HSV colour space, both parameters saturation and value must be above a certain threshold. However, we could get rather good results by using a local filter which is just based on the value parameter of the HSV colour space. Therefore, we will focus on the HSV colour space and in the following all given parameters, hue, saturation and value, will correspond to this colour space.

As an example, a synthetic image with a different coloured particles and background noise is generated. It is shown in figure 7.9 (a). The background noise is set to $0.1 \in[0,1]$, the particles with a size of $3 \times 3 \mathrm{px}$ are placed on top of the noise and have a linearly decreasing intensity from left to right from 0.9 to 0 . Thus, close to the right side of the image the particles vanish within the noise. Such a configuration is chosen to see how robust the filtering algorithm is with respect to the signal to noise ratio. The colours of the particles are red at the top, then passing to yellow, green, blue and purple, until they become red at the bottom again. Therefore, the hue increases from top to bottom from $H=0$ to $H=1$.

Figure 7.9 (b) presents the pixelwise calculated hue map of the test image. One finds a random distribution of all hue values, clearly showing that the hue can not directly be used for the calculation of the colour and therefore the temperature distribution.

The next possible step is to apply a median filter. The result for a $16 \times 16 \mathrm{px}$ median filter is shown in figure 7.9 (c). The tendency of increasing hue from top to bottom is visible now, nevertheless all mean hue values are in the vicinity of $H=0.5$. This is caused by the impact of the background noise with random hue values and clearly shows that an image which has more background than particles can not be simply evaluated by calculating the mean hue within interrogation windows.

The idea is to use interrogation windows again, but to take only those pixels into the calculation of the mean hue value for this interrogation window that are not background noise. Due to slightly inhomogeneous illumination in almost all experimental setups, we applied such a filter with a local rather than a global threshold for the identification of particles. Therefore, we chose the $V$ value, i.e. the brightness, (see figure 7.9, d) of the colour 
image and localise those pixels where the value of $V$ has increased from the background level. Within each interrogation window, we take only those pixels into account for the calculation of the mean hue for the whole interrogation window, where the highest $V$-values are located. To clarify this procedure, figure 7.9 (e) shows a scatter plot of hue vs. $V$-value for a $16 \times 16 \mathrm{px}$ interrogation window. For low values of $V(V \lesssim 0.1)$, one finds all $H \in[0,1]$, but for high values of $V$, only a rather sharp hue distribution appears. We identify those pixels as the particles and the rest as the background noise. For the calculation of the mean hue, we take into account only the pixels with the highest value of $V$, which are marked with a box; in this example the threshold was set to four. Both the size of the interrogation windows as well as the threshold for how many pixels should be taken into account must be adjusted to the particle density of the image.

Applying this procedure to the synthetic image results in figure 7.9 (f). One can see three things: firstly, the hue gradient from almost $H=0$ at the top to almost $H=1$ at the bottom can be reproduced. Secondly, even at a very low signal to noise ratio, on the right side of the image, the algorithm reproduces the hue values correctly. Thirdly, in red regions where $H \approx 0$ or $H \approx 1$, some errors are produced due to fact the hue is periodic and that a pure red colour is either $H=0$ or $H=1$. However, for the calibration of the liquid crystals this fact should not be bothersome, because the liquid crystals turn from red to yellow and green to blue until they become colourless again, meaning that $H \approx 1$ will not appear using liquid crystals.

Although filtering can be realised in a similar way in the HLS colour space, filtering with the $V$-value in the HSV colour space yields good results for almost all investigated particle images so far, so we concentrate on this technique for now.

\subsubsection{Dynamic Calibration}

To obtain the absolute temperature information from the hue values, a hue-temperature calibration must be conducted. The calibration function found is only valid for the specific setup, i.e. only for the specific arrangement of cameras and light-sheet optics as well as for the crystal type and the particle generator used. Typically, during periods of stable layering of the temperatures, the different hue values and the corresponding temperatures are recorded. Due to the difficulty of generating these stable temperatures within our geometry and due to the high time effort of this calibration technique, we decided to apply, as a fast approach, a dynamic calibration to correlate the recorded hue values to the absolute temperatures. The main advantage is the rapidity of the calibration process. Therefore, a very quickly reacting, precisely calibrated glassbed NLC thermistor with a diameter of only $400 \mu \mathrm{m}$ is placed within the measurement plane. Using this thermistor, which is calibrated with an accuracy of $\sigma_{\mathrm{T}, \text { therm. }}=0.05 \mathrm{~K}$, the temperatures are recorded at a high frequency (compared to the recording frequency of the PIT/PIV images) of approximately $13 \mathrm{~Hz}$. For the hue-temperature calibration, the hue values are evaluated as described in the previous section and those hue values recorded next to the position of the thermistor are correlated 
to the temperatures. This results in time series for hue and temperature, as shown in figure 7.10(a). By eye, one can already see that the two quantities correlate with each other. It must be noted that the response time of the crystals is much lower than that of the $400 \mu \mathrm{m}$ thermistor, whose thermal inertia acts as a low pass filter and therefore averages the high frequent temperature fluctuation caused by turbulence.

Plotting the temperature as a function of the hue value confirms this, see figure 7.10 (b). A linear fit to the measured data results in $T\left[{ }^{\circ} \mathrm{C}\right]=m \cdot H+b=14.8( \pm 0.7) \cdot H+$ $18.5( \pm 0.1)$. The fluctuations of the signal incorporate different contributions. However, the main part is ascribed to the different reaction times of the thermistor and the TLCs. The latter are able to react to the temperature changes in the turbulent flow, i.e. the TLCs register more temperature fluctuations than the thermistor. However, the resulting uncertainty of the calibration parameters can be easily reduced by extending the calibration measurements, because the uncertainty of the mean hue value in a certain hue interval $\sigma_{<H>}$ scales like $\sigma_{<H>}=\sigma_{H} / \sqrt{n}$, where $\sigma_{H}$ is the standard deviation of the hue values in the interval and $n$ is the number of recorded values. Using the fit errors of the linear regression, the uncertainty of the thermistor and the Gaussian error propagation for our actual calibration measurement, a hue depending calibration uncertainty of $\sigma_{T_{\text {cal,min }}}=$ $\sqrt{\left(\sigma_{m} \cdot H_{\min }\right)^{2}+\sigma_{b}^{2}+\sigma_{\mathrm{T}, \text { therm }}^{2}}=0.15 \mathrm{~K}$ at $H_{\min }=0.14$ is found, which increases to $\sigma_{T_{\text {cal }, \text { max }}}=\sqrt{\left(\sigma_{m} \cdot H_{\max }\right)^{2}+\sigma_{b}^{2}+\sigma_{\mathrm{T}, \text { therm }}^{2}}=0.18 \mathrm{~K}$ at $H_{\max }=0.21$. The corresponding error interval is indicated in figure 7.10 (b) by the dashed lines.

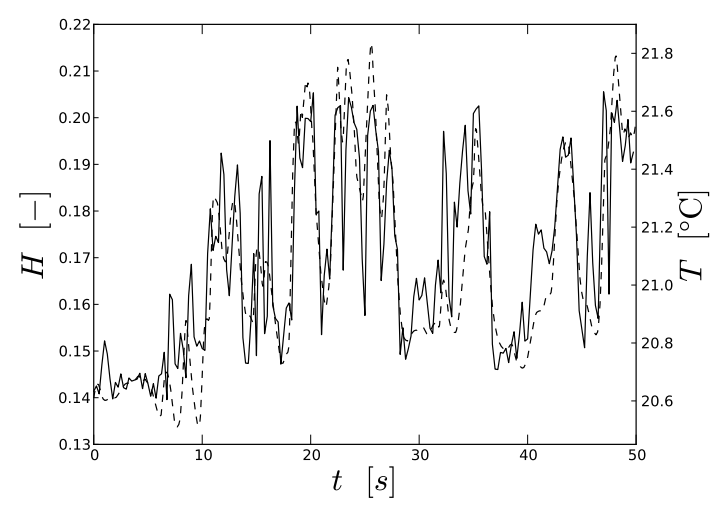

(a)

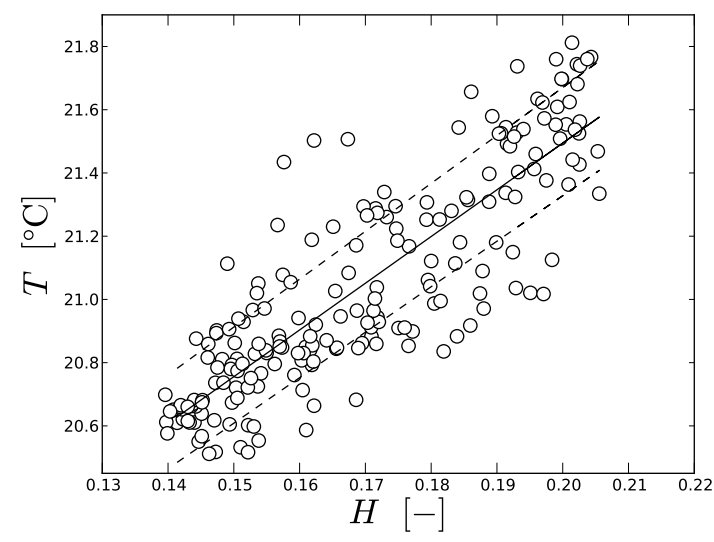

(b)

Figure 7.10: (a) Time series of simultaneously recorded hue (-, left axis) and temperature (--, right axis). (b) Correlation of temperature and hue value, including a linear fit $(-): T\left[{ }^{\circ} \mathrm{C}\right]=14.8( \pm 0.7) \cdot H+18.5( \pm 0.1)$ including its error interval $(--)$.

However, these uncertainties are valid for absolute temperature measurements only. The error of relative temperature measurements due to the uncertainty of the calibration, following the Gaussian error propagation, is much smaller and given by $\sigma_{\Delta T_{\text {cal,max }}}=$ $\sqrt{\left(\sigma_{m} \cdot \Delta H_{\max }\right)^{2}}=0.05 \mathrm{~K}$. For the actual measurement, however, other noise sources, which can be expected to average out during calibration, come into play and have to be 
considered. Most noteworthy for PIT is the pixel-hue noise, which is the variation of the hue value in a single interrogation window at an imaginary constant temperature. This error contribution can be caused by colour-noise of the PIT-camera, colour-noise of the light source and the size distribution of the TLC particles, whose colour play is known to depend on the actual particle size. Obviously, this noise can be decreased at the expense of spatial resolution by enlarging the interrogation window size. For an interrogation window size of $12 \times 12 \mathrm{px}$ in combination with a threshold of 64 , i.e. the settings used in the following, this error has been estimated to amount to $\sigma_{\text {hue }} \approx 0.0016$, as described in the following. As already stated in section 7.2.1, the smallest coherent structures to be expected in our flow measure around $10 \mathrm{~mm}$, while the dimensions of our interrogation windows for PIT are as small as $5 \times 5 \mathrm{~mm}$. Accordingly, the hue fluctuations of a single interrogation window reflect measurement noise only. In order to determine this value, we computed the mean spatial standard deviation of the measured hue values in quadratic conglomerates of adjacent interrogation windows, which we averaged over the whole field of view and the whole image series. The results are depicted in figure 7.11. In order to determine the standard deviation of the hue value for a single interrogation window, the measured data was fitted with a power law and extrapolated to the value for $n_{\text {int.win }}=1$, which is then interpreted as the hue noise error of a single interrogation window.

Using again Gaussian error propagation, we obtain $\sigma_{T_{\max }}=\sqrt{\left(\sigma_{m} \cdot H_{\max }\right)^{2}+\sigma_{b}^{2}+}$ $\overline{+\sigma_{\text {T,therm }}^{2}+\left(\sigma_{\text {hue }} \cdot m\right)^{2}}=0.19 \mathrm{~K}$ and $\sigma_{\Delta T_{\max }}=\sqrt{\left(\sigma_{m} \cdot \Delta H_{\max }\right)^{2}+2 \cdot\left(\sigma_{\text {hue }} \cdot m\right)^{2}}=0.06 \mathrm{~K}$ as maximal values for the absolute and the relative error, respectively. This results in a dynamic range for the actual measurement of $\left(H_{\max }-H_{\min }\right) / \sigma_{\text {hue }} \approx 43$.

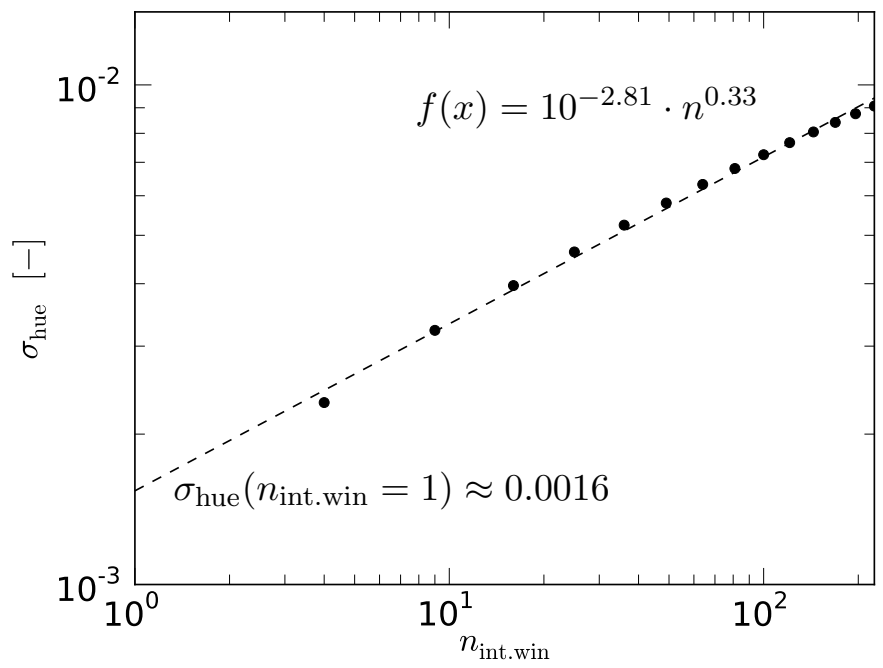

Figure 7.11: Estimation of the hue error for an interrogation window size of $12 \times 12 \mathrm{px}$ using a threshold of 64 .

At this point it should be noted though, that the calibration described above has to be improved in the next steps by incorporating more measurement data, considering the impact of different viewing angles in the same field of view and systematically investigating 
the use of the evaluation parameters, which influence and can thus be used to optimise bandwidth and the dynamic range.

Other filtering and calibration procedures, as proposed in the literature, e.g. Rao and Zang [85] proposing a $5 \times 5$ median filter, Grewal et al. [47] introducing neural networks, and Roesgen and Totaro [91] suggesting a statistical technique using a proper orthogonal decomposition, are promising approaches to further improve the accuracy of the technique.

\subsection{Experimental Testing and Analysis}

\subsubsection{EXPERIMENTAL Setup}

The investigated mixed convection cell has a square cross section of $500 \mathrm{~mm} \times 500 \mathrm{~mm}$ and an aspect ratio between length and height of $\Gamma_{\mathrm{xz}}=5$ (see figure 7.12, a). The air inflow is supplied through a slot below the ceiling, while exhaustion is provided by another slot above the floor. Both vents extend over the whole length of the cell. The bottom and ceiling of the cell can be maintained at different temperatures. This setup allows generating mixed convection under well defined conditions, and serves as a generalised model system for geometrically more complex technical configurations. The flow field and the heat transport within this container have already been presented in some publications: Westhoff et al. [140] investigated the low-frequency oscillations of the large-scale circulation, Kühn et al. [66 applied a newly developed tomographic PIV technique to large volumes, and Schmeling et al. 105 studied the large-scale flow structures and the heat transport using PIV and temperature probes. Again Schmeling et al. [98] investigated the large-scale circulations and their oscillations by means of many temperature probes within the cell combined with smoke visualisations.

For the first experiments with the combined PIT/PIV technique, no external pressure gradient is applied between the inlet and the outlet of the enclosure. Thus, the measurements are conducted rather in pure thermal convection than in mixed convection. Nevertheless, due to the still existing openings of both the inflow and outflow channels, a slow fluid exchange with the surrounding takes place. The fields of view with extensions of approximately $300 \times 150 \mathrm{~mm}^{2}$ for PIT and $225 \times 150 \mathrm{~mm}^{2}$ for PIV are located close above the heated bottom plate and close to the front side of the enclosure, see figure 7.12 (b) and the coordinate systems in figure 7.16, where $y=0$ and $z=0$ is the lower front edge of the enclosure. The difference of the sizes of the fields of view is caused by the different angles under which the cameras are operated. Remapping and superposing the images is realised based on a well known technique for stereoscopic PIV (e.g. [84]) using recordings of a calibration grid.

The TLC particles are generated with the airbrush system, which is supposed to inject the particles into the settling chamber in front of the inlet for mixed convection cases. During the feasibility tests, as already mentioned, the external volume flow is switched off in order to provide a longer retention period in the cell, therefore, the particles have to be sprayed directly into the enclosure. For illumination of the particles, the specially developed 


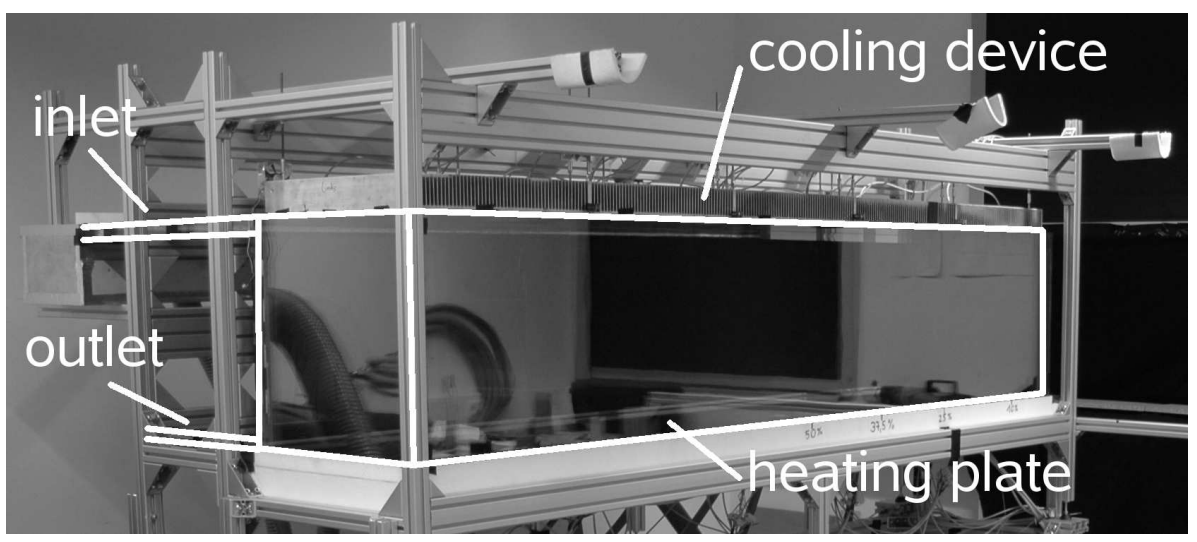

(a)

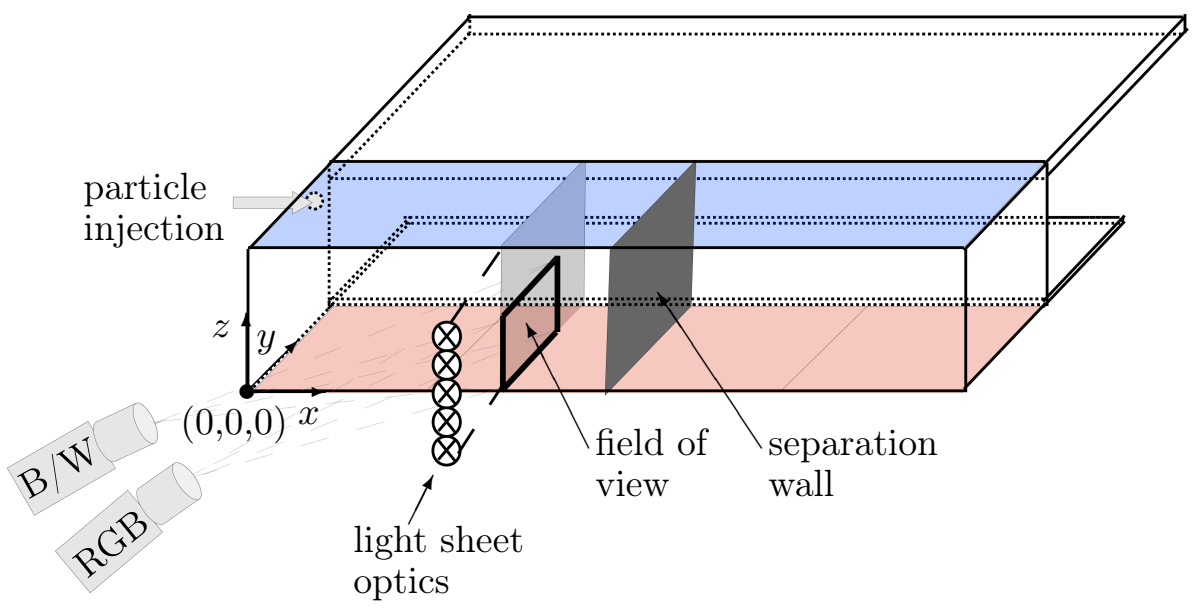

(b)

Figure 7.12: (a): Picture of the mixed convection cell showing the heating plate at the bottom, the cooling plate at the top, as well as the slots for air in- and outflow at the side. (b): Sketch of the experimental setup used for combined PIT and PIV, including the coordinate system and the origin.

white light sheet based on white LEDs is used. The particle images are recorded with a double shutter grey level and a single shutter colour CCD camera, so that temperatures could be determined from the local hue values and velocities from the local particle image displacements between subsequent recordings. The use of two cameras provides better results than using just a single double shutter colour CCD camera. This is because the less sensitive colour camera can be operated in async. shutter mode, recording only the first of the two light pulses, while the highly sensitive grey level camera is operated in the double shutter mode for the PIV evaluation. Furthermore, the signal to noise ratio of the grey level camera is lower than that of the colour camera, which leads to a higher quality of the PIV results. 


\subsubsection{Results I: QUALITY OF THE RESUlts}

A raw particle colour image is presented in figure 7.13 (a), to depict the quality and the contrast of the colour images used for the temperature evaluation. A plume of warmer air can already be seen in this picture: the blue part in the centre indicates higher hue values than those of the green and red surroundings. Both frames of the $\mathrm{B} / \mathrm{W}$ image after high and low pass filtering are shown in figure 7.13 (b). Further, information on the applicability of PIV is given in figure 7.14, which presents (a) the correlation plane (left) for one interrogation window $(24 \times 24 \mathrm{px})$, including the corresponding windows of the two frames (middle and right). The size of the particle images, which is approximately $2 \times 2$ to $3 \times 3 \mathrm{px}$, the suitable number density of particle images per interrogation window, and the clear peak in the correlation plane indicate the high quality of the PIV measurements. Additionally, figure 7.14 (b) reflects the 2D and (c) the 1D pixel displacement distributions; these depict the good quality of the PIV results in terms of the absence of peak locking.
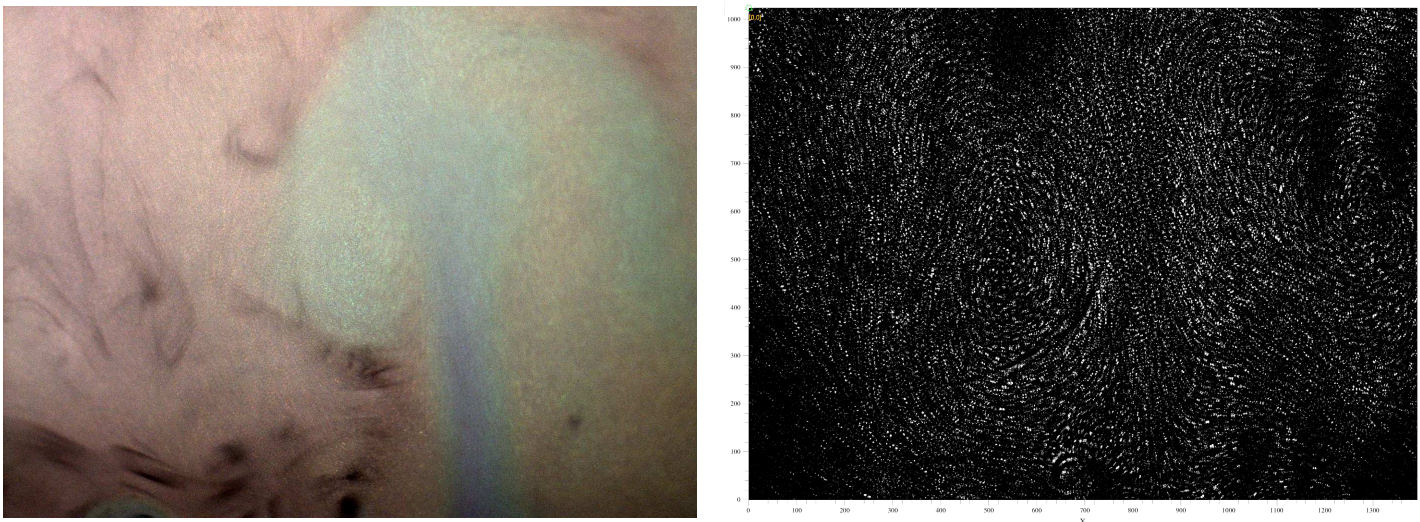

Figure 7.13: (a) Raw colour image, (b) both frames of the B/W image after high and low pass filtering. Note that the images are unrectified and do not completely overlap. A warmer plume can already be seen in this picture: the blue part in the centre indicates higher hue values than the green: these increased hue values indicate an increased temperature.

After rectifying, overlapping and evaluating the data, simultaneously recorded instantaneous temperature and velocity fields as depicted in figure 7.15 can be calculated. The formation of a warm plume which rises from the lower thermal boundary layer can be seen. The top hat of the plume as well as both side vorticities and the root with quickly rising warm air are clearly visible. Such a detailed recorded picture of temperature and velocity of a rising thermal plume has, as far as we know, not been previously recorded experimentally.

For the velocity calculation, we use a pulse delay of $\tau=16 \mathrm{~ms}$ at a light pulse length of $1 \mathrm{~ms}$, which leads to a pixel displacement up to $10 \mathrm{px}$ and therefore a maximal particle shift during one light pulse of less than $\Delta x \approx 0.7 \mathrm{px}$. Furthermore, we use an interrogation window size of $24 \times 24 \mathrm{px}$, an overlap of $50 \%$, a three point Gaussian fit for the peak detection, and a seven step multigrid evaluation starting at a window size of $256 \times 256 \mathrm{px}$ and disabled sub-pixel image shifting. This results in a vector spacing of approximately 

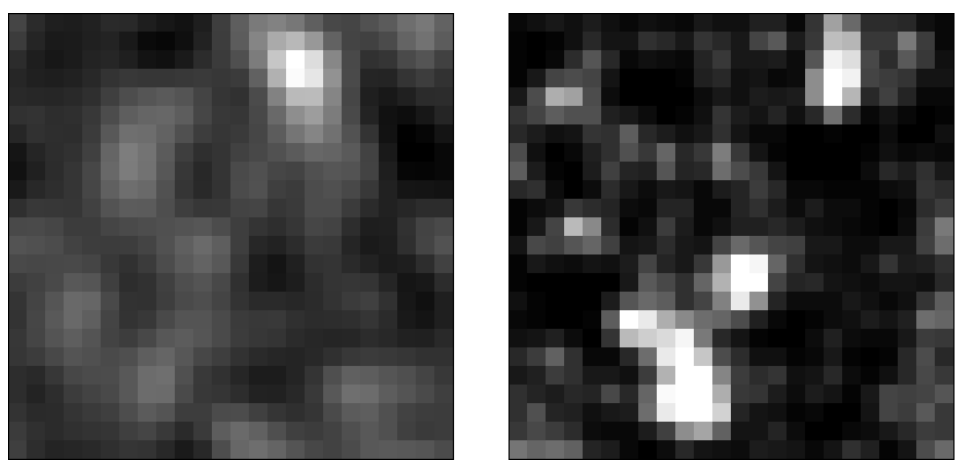

(a)
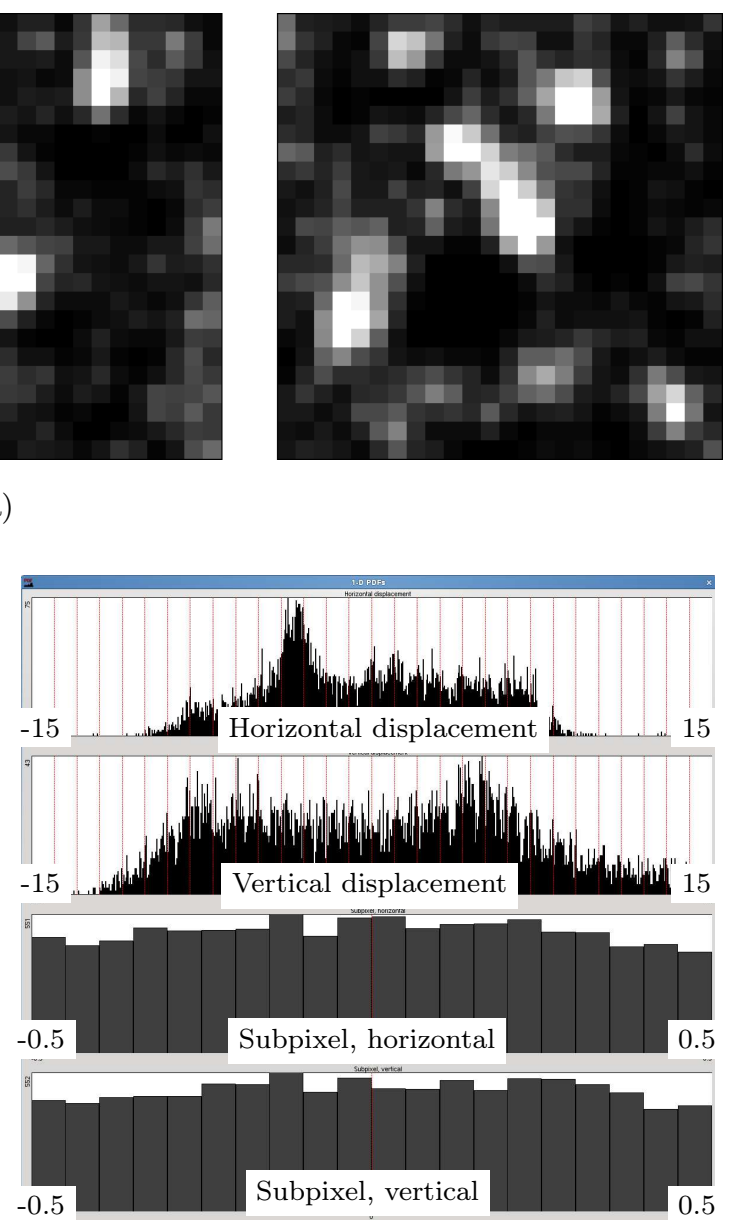

(c)

FIGURE 7.14: (a) Left: correlation plane, middle: first image, right: second image. (b) 2D displacement plane and (c) 1D displacement histograms.

$2.5 \mathrm{~mm}$. For the sake of clarity, only every $2^{\text {th }}$ vector in each direction is shown in figures 7.15 and 7.16 . The hue values, i.e. the temperature values as well, are calculated using a filter size of $12 \times 12$ pixel and a threshold of 64 , see section 7.2 .4 .

To conclude this subsection, a short discussion of possible errors and limitations resulting from the use of TLC tracer particles, an LED based white light illumination, as well as the camera systems will be given. First to be mentioned are the spatial homogeneity of the particle distribution, as in any PIV evaluation. This is already rather good, see e.g. the particle images in figure 7.13 (b). The homogeneity of the illumination, which is also no problem for this field of view, but might be a more important issue with larger measurement planes due to the divergence of the LEDs. The light sheet thickness, which is approximately $10 \mathrm{~mm}$, is in reasonably good agreement with the interrogation window size used for PIV $(\approx 5 \times 5 \mathrm{~mm})$, but imposes a restriction on the spatial resolution for non purely $2 \mathrm{D}$ flows. These points may cause some errors and limitations, but do not influence the results in our studies negatively so far. Secondly, there are restrictions regarding the 

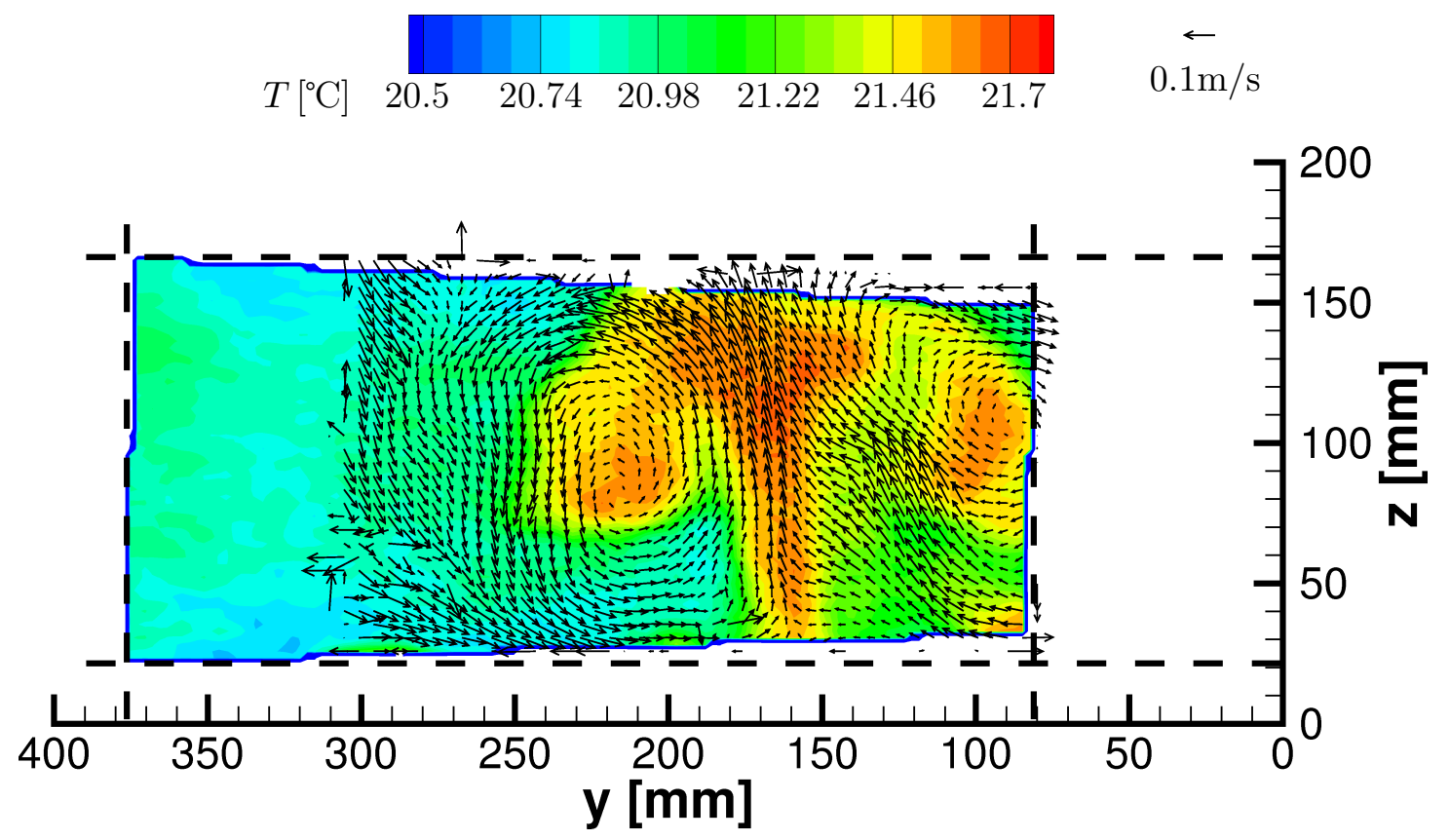

Figure 7.15: Instantaneous temperature contours and velocity vectors for the above shown raw particle images. For the sake of clarity, only every $2^{\text {nd }}$ vector is shown in each direction. Recorded at $\operatorname{Pr}=0.71, R e=0$ and $R a \approx 9.0 \times 10^{7}$. The colour bar reflects the relative accuracy of $\sigma_{\Delta T}=0.06 \mathrm{~K}$. The error of the absolute temperatures is $\sigma_{T}=0.19 \mathrm{~K}$.

maximal flow velocity that can be studied. These are the following behaviour of the tracer particles, which is discussed in detail in section 7.2.2. The duration of the light pulse, which limits, in combination with the imaging optics used, the maximal flow velocity as well, because particle images would be smeared out or stretched to stripes for too long pulses in combination with fast flows. In our system, a pulse duration of $1 \mathrm{~ms}$ still leads to sharp particle images, see e.g. the particle images in figure 7.13 (b) and 7.14 (a). Thirdly, the accuracy of the temperature and the sensitivity of the hue measurements have been analysed and discussed in section 7.2.5. Currently, the achievable accuracy in the temperature measurement is limited by the accuracy of the hue temperature calibration, while the hue sensitivity would offer more headroom. Nevertheless, relative temperatures can already be measured with an accuracy of $\sigma_{\Delta T_{\max }}=0.06 \mathrm{~K}$, which is reflected in the colour bar of the contour plots of our results accordingly.

\subsubsection{Results II: Flow ANALysis}

The convective air flow investigated here is induced by a temperature gradient between the bottom and the top plates of $\Delta T=7.2 \mathrm{~K}$, while the external pressure gradient is set to zero. This results in the following characteristic numbers: Rayleigh number $R a \approx 9.0 \times 10^{7}$, Reynolds number based on the inflow velocity $R e=0$ and Prandtl number $\operatorname{Pr}=0.71$. Thus, except for the unsuppressed fluid exchange through the openings of the inlet and the 


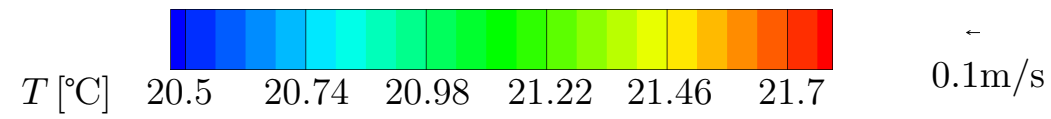

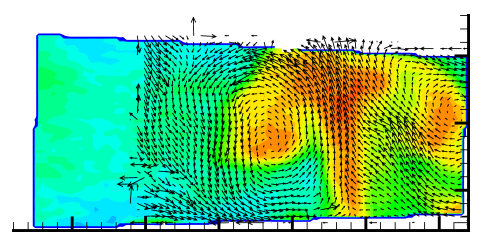

(a)

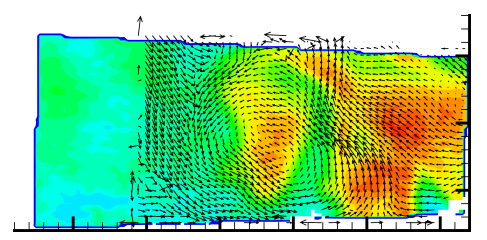

(d)

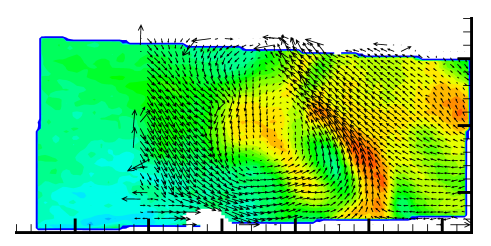

(g)

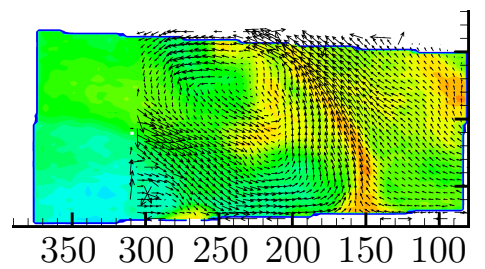

(j)

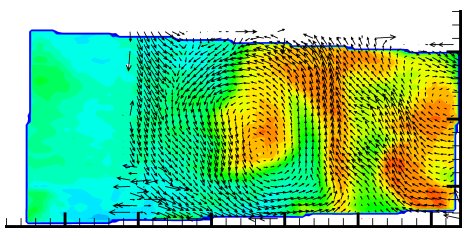

(b)

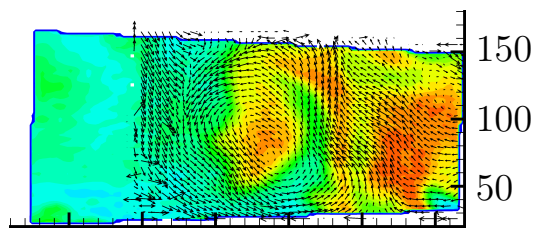

(c)

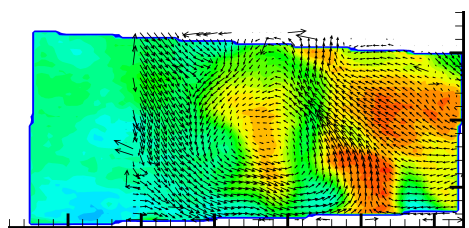

(e)

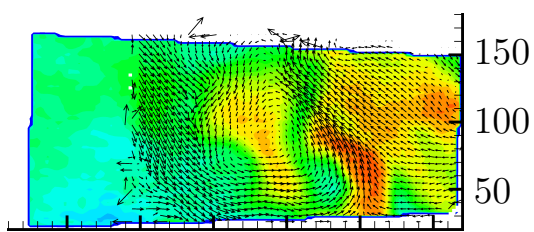

(f)

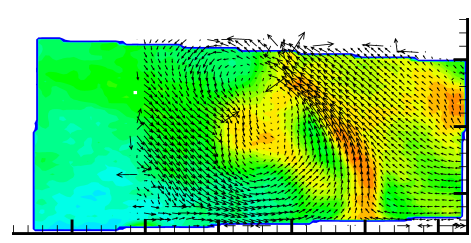

(h)

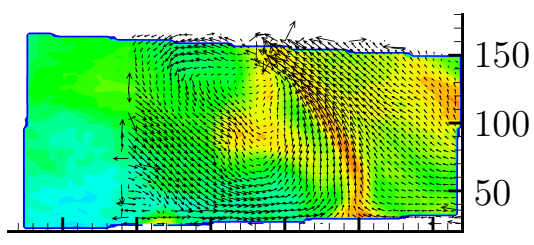

(i)

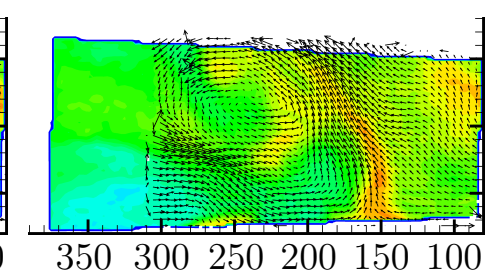

(k)

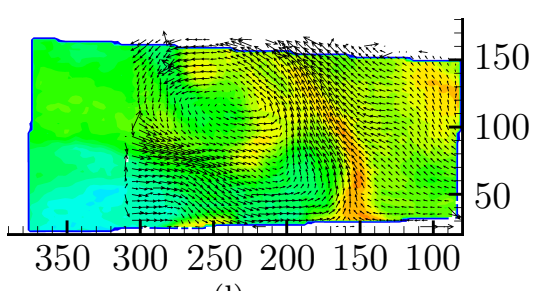

(1)

FiguRE 7.16: Instantaneous temperature contours and velocity vectors for twelve instants in time ( $\Delta t=0.25 \mathrm{~s}$ between two images) showing the dynamics within the enclosure. For the sake of clarity, only every $2^{\text {nd }}$ vector is shown in each direction. The legend and the reference vector at the top apply to all images. The colour bar reflects the relative accuracy of $\sigma_{\Delta T}=0.06 \mathrm{~K}$. The error of the absolute temperatures is $\sigma_{T}=0.19 \mathrm{~K}$.

outlet, pure thermal convection is investigated.

The results of simultaneous PIT and PIV measurements in our rectangular convection cell are presented in figure 7.16, which shows the temperature as contour and velocity, calculated from image pairs by means of well established PIV algorithms [84, as a vector field. Thereby, the maximal observed velocity is approximately $0.12 \mathrm{~m} / \mathrm{s}$. The images are recorded at a recording frequency of $4 \mathrm{~Hz}$. Figure 7.16 shows twelve consecutive images, i.e. the time difference between two images is $0.25 \mathrm{~s}$ and the series spans a duration of $3 \mathrm{~s}$. A supplemental movie created out of 400 single images at a recording frequency of $4 \mathrm{~Hz}$, i.e. spanning a time period of approximately $100 \mathrm{~s}$ is available online 99]. 
The parameters used for the PIV and PIT evaluation are the same as described in the previous section, besides the fact that now only every $2^{\text {th }}$ vector is shown in each direction for the sake of clarity in figure 7.16 and every $3^{\text {rd }}$ in the movie. Finally it should be noted that no outlier detection is used during the PIV interrogation, but for the visualisation, especially in the supplemental movie, a value blanking using a threshold for the velocity is applied to remove distracting vectors.

Figure 7.16 (a) is again the warm plume which was already depicted and described in the section above (figure 7.15). The time series of images here (a) to (l) shows the breakdown of the typical plume structure towards a thin region of warm rising air at a rather high time resolution of $4 \mathrm{~Hz}$. Thereby, the vortex on the left side of the plume is almost at rest during these $3 \mathrm{~s}$, while the right one convects out of the field of view, leading to the breakdown of the typical mushroom structure. Due to the fact that the velocity and temperature fields are recorded as 2D fields in a plane, it must be taken into account that there will be an out-of-plane component which is not recorded here.

Nevertheless, from the fluid dynamical point of view, the instantaneous temperature and velocity fields recorded at a high frequency open new possibilities in, e.g. analysing the correlation between these two quantities and studying their time evolution. Furthermore, simultaneous measurements will help to further investigate the dynamical processes which lead to, e.g. reorientations and oscillations within convective flows observed, amongst others, in our convection cell $[98]$.

\subsection{SUMMARY AND CONCLUSION}

We performed combined Particle Image Thermography (PIT) and Particle Image Velocimetry (PIV) in convective air flow using thermochromic liquid crystals (TLCs) as tracer particles. The technique allows for simultaneous recording of planar instantaneous temperature and velocity fields. In order to accomplish our measurements, different ways of particle generation were studied and a suitable white light sheet optics was developed. The latter generates a white light sheet with a thickness of less than $10 \mathrm{~mm}$ over a distance of $500 \mathrm{~mm}$. It is based on Light Emitting Diodes (LEDs), which guarantee a fast response within less than $50 \mu$ s to the trigger signal at rather low cost.

In order to separate the temperature information from the background and pixel noise, a filtering procedure of the recorded colour data was developed. It is based on averaging the hue over interrogation windows while taking into account only those pixels with a $V$ value (HSV) above a certain local threshold. Its working process, applicability and benefits are demonstrated on a synthetic image.

Results from application of the technique to mixed convection in a cuboidal container were presented and discussed. Amongst others, a thermal plume including its mushroom structure, the adjacent vortices, and its root with quickly rising warm air could be captured with a high spatial resolution. Furthermore, due to a time resolution of $4 \mathrm{~Hz}$, a large fraction of its dynamics could be resolved. 
In order to perform quantitative measurements, a fast calibration procedure has been developed and employed which provided a calibration accuracy of the absolute temperatures of $\sigma_{T}=0.19 \mathrm{~K}$ in the studied temperature range. By considering the errors of the calibration curve and the hue noise, which has been computed from the measurement data, a standard error for relative temperature measurements as low as $\sigma_{\Delta T}=0.06 \mathrm{~K}$ was calculated, corresponding to a temperature dynamic range based on the investigated temperature interval of 20. The dynamic range on the hue scale, however is much higher and amounts to 43 , revealing an even higher potential of the technique, depending on the accuracy of the hue temperature calibration.

The next steps in further maturing the technique therefore incorporate further research on the hue-temperature calibration in order to push the limits in terms of increased precision as well as the optimisation of the measurement and evaluation parameters for higher dynamic ranges for the hue values. Other issues, which have to be addressed to help to establish the technique are further characterisation TLC particles in air flow. Here we think especially on the colour play, which is known to deviate for dispersed TLC particles from the bulk properties. Knowledge and control of the size dependence of the colour play could help to optimise the measurement and dynamic range in the future. Also measurement of the exact reaction time of the particles are required to assess the potential of the technique to application in terms of higher velocities.

\section{ACKNOWLEDGEMENTS}

The authors are grateful to Daniel Schiepel for his ideas on the setup of the new lightsheet optics. 


\title{
8 Dynamics of Thermal Plumes in Turbulent MC 100
}

Citation and credit: in preparation for Experiments in Fluids

Reference: 100

Title: "Measurements of the dynamics of thermal plumes in turbulent mixed convection based on combined PIT and PIV"

Authors: Daniel Schmeling, Johannes Bosbach and Claus Wagner

Contributions: I performed all measurements, analysed them and made all figures. I wrote the first draft, whose structure has been preserved.

\begin{abstract}
The dynamics of thermal plumes and their abundance is investigated in mixed convection in a cuboidal sample with respect to the characteristic numbers. The parameter range spans $R a=1.0-3.2 \times 10^{8}, R e=0.5-1.7 \times 10^{4}$ and $A r=1.1-7.6$. Combined Particle Image Thermography (PIT) and Particle Image Velocimetry (PIV) is conducted in a horizontal layer close above the bottom thermal boundary layer. This combination of measurement techniques, using thermochromic liquid crystals (TLCs) as tracer particles, which is novel for air flows, allows for simultaneous measurement of temperature and velocity fields. Details of the measurement technique are published in 101.

The fingerprints of sheet-like plumes and those of the stems of mushroom like plumes are visible in the instantaneous temperature fields. A study of temperature PDFs reveals, that the distributions can be well described by a sum of two Gaussian distributions. Analysing the ratio of the probabilities $P_{2} / P_{1}$ reveals a sudden change at a critical $R a_{c} \approx 2.3 \times 10^{8}$. Here $P_{1}$ denotes the abundance of fluid temperatures imprinted by the bulk flow, while $P_{2}$ represses the abundance of temperatures ascribed to warm thermal plumes. Accordingly, $P_{2} / P_{1}$ is a measure for the plume fraction in the measurement plane. The change occurs in
\end{abstract}


the $A r$ regime $2.7 \lesssim A r \lesssim 3.3$, in which the interaction of buoyancy induced large scale circulations (LSCs) with the wall jet of the incoming air results in an instability reported already by 98 .

A combined evaluation of the temperature and velocity fields reveals a change of the horizontal heat fluxes is found at $A r \approx 2.7-3$. Furthermore, the total amount of heat transported horizontally within the measurement layer increases with $R a$ in bulk dominated regions, while it stays almost constant for plume dominated ones.

A flow reversal in the measurement plane occurs as a function of $A r$. At low $A r$ the angle between the mean flow direction and the $x$-axis amounts to approximately $120^{\circ}$. An intermediate $A r$ results in an angle of $18^{\circ}$, which we ascribe to the interactions of buoyancy with the wall jet. At higher $A r$ we find an angle of approximately $-75^{\circ}$. Moreover, no unique dependency of the time averaged angle on $R e$ or $R a$ could be observed.

\subsection{INTRODUCTION}

Convective flows, in specific Rayleigh-Bénard convection (RBC), are abundantly addressed in scientific studies. For detailed reviews see [3, 30,71. Onwards, the occurrence of convection in nature and in technical applications is also tackled in many publications, see e.g. 17, 34, 69, 110, 120.

Thereby, it is well known, that the thermal plumes in convection play a central role for heat transport and structure formation in TC. Accordingly, their investigation augurs significant progress in the understanding of the physical processes in convective flows. Measurement techniques similar to ours were already applied to turbulent RBC by Zocchi et al. [154 using water as working fluid, at $\operatorname{Pr} \approx 6$. They used thermochromic liquid crystals (TLCs) to visualise the temperature fields in a horizontal layer in the lower thermal boundary layer and a vertical layer close to the cold top ceiling of the sample. Herein, the visualisations showed the fingerprints of thermal plumes and sheet-like plumes, swirling of them and the impact of cold plumes hitting the warm boundary layer at the bottom. From the observation Zocchi et al. depicted a "life cycle" within the sample (see Fig. 3 in [154]). This life cycle comprises that plumes, which hit the opposing boundary layer excite waves therein. These waves travel as sheet-like plumes along the boundary layer with the mean wind. Meanwhile, mushroom like plumes and swirls develop out of the line plumes. While travelling with the wave, they grow until they detach and travel through the bulk of the sample towards the other boundary layer.

More recently, the role of plumes for the onset of the large-scale circulation (LSC) was studied by Xi et al. [147], who stated that the thermal plumes initiate the horizontal largescale flow. This was concluded from shadowgraph images and velocity field measurements. Similar shadowgraph images, which show the spatial distribution of the thermal plumes, were recorded by Shang et al. 146 at $\operatorname{Pr}=596$. From combined temperature and velocity measurements they concluded, that the heat in turbulent convection is carried by the thermal plumes. Furthermore, the local heat flux was found to be highly intermittent, 
which is associated with the dynamics of the plumes.

A deeper analysis of the individual sheet-like plumes reveals that their area, circumference and amount of heat exhibit log-normal distributions 152 . Visualisations of temperature fields of the plumes by using TLCs and accompanied velocity measurements in a cylindrical water convection sample resulted in these findings. By analysing planes in different heights, the authors concluded that the sheet-like plumes collide and convolute to swirls when moving over the heated plate. These swirls become mushroom like plumes when they move away from the plate. The alignment of the sheet-like plumes, even though they did not name them so, parallel to the mean wind in the convection sample, was already observed by [51]. Parallel streaks were found within the bottom thermal boundary layer in thermal convection (TC) and different possible formation mechanisms were discussed.

For a quantitative analysis of the plumes and the impact of the latter on e.g. the heat transport or the large-scale flow, criteria for the detection are required. One possibility for extracting sheet-like plumes from data generated in Direct Numerical Simulations (DNS) was presented by Shishkina and Wagner [108]. They proposed a temperature threshold to distinguish between the plumes and the turbulent background. The application of this criterion showed that sheet-like plume are characterised by relative large vertical velocities and high values of local heat flux. Moreover, the highest values of these quantities are found in regions where the sheet-like plumes collide, swirl up and form the stems of mushroom like plumes, which develop into the bulk of the convection sample.

Recently, Emran and Schuhmacher [37] proposed a criterion based on both, temperature fluctuations and vertical velocity, in order to decompose the sample volume into a plume dominated part and the turbulent background part. They found that the plume dominated part is not only limited to the thermal boundary layers, but instead extends over the whole sample volume. They state, that the subvolume of the plume dominated part forms a skeleton through which the heat is transported through the sample.

It should be noted that all mentioned criteria for plume detection are either purely qualitative, use a threshold of the temperature or depend even on temperature and velocity fields. Hence, the experimental acquisition of both quantities is highly desirable.

Further studies of the normalised length of sheet-like plumes close to the heated bottom plate were performed by Puthenveettil et al. [83. For three different $\operatorname{Pr}$ the normalised length of the plumes per unit area was found to remain constant for a single fluid and varying $R a$. Furthermore they showed that the dimensionless heat transport, i.e. $N u$, is directly proportional to the the length per unit area times the height of the sample.

Recently, plume fragmentation could be ascribed to interactions with the turbulent background and domain walls 18 . They investigated sheet-like plumes and their fragmentation in turbulent $\mathrm{RBC}$ using the shadowgraphy technique, see for instant [35]. In a convection sample of aspect ratio $\Gamma \approx 10$, using compressed gases at $\operatorname{Pr} \approx 0.7$ as working fluids, statistics of the plume lengths are analysed. The observed log-normal distribution as well as the scaling of the total plume length with $R a$ revealed that the length distribution is determined by fragmentation caused by interactions with turbulent bulk fluctuations and 
domain walls. Regarding these findings, the impact of additional shear, i.e. mixed convection as investigated in the current study, which causes distinguished velocity fluctuations in the bulk, opens new perspectives.

Concluding this introduction, it shall be noted that the investigations of the plume dynamics in air were so far conducted based on numerical simulations or qualitative visualisation techniques. Accordingly, the measurement of temperature fields, absolute or just relative, in air in a layer parallel and as close as possible to the bottom thermal boundary layer, would allow for new insights in the involved physical mechanisms. Finally, performing and analysing all these measurements in a system with additional shear forces, namely mixed convection (MC), will help to expand the knowledge of thermal plumes and their interactions with bulk fluctuations.

\subsection{Experimental Setup AND Procedure}

\subsubsection{Convection SAmple}

MC is studied in a cuboidal convection sample of length $L=2500 \mathrm{~mm}$ with the aspect ratios $\Gamma_{\mathrm{xz}}=\frac{L}{H}=5$ and $\Gamma_{\mathrm{yz}}=\frac{W}{H}=1$, where $H$ denotes the height and $W$ the width of the sample. A sketch of the sample is shown in Fig. 8.1. The bottom of the sample is heatable, while the ceiling is kept at ambient temperature. The side panels are a double-wall system of polycarbonate plates, which guarantee good optical access as well as minimal heat loss. By operating the heating plates, a vertical temperature gradient is generated to induce TC. Furthermore, the cell is equipped with an air inlet and an air outlet located at the top and at the bottom of one side wall, respectively. The height of the inlet and the outlet channels amounts to $25 \mathrm{~mm}$ and $15 \mathrm{~mm}$, respectively. Both are located on the same side wall and extend over the full length of the container. This configuration allows to generate a flow between the in- and outlet, driven by an applied pressure gradient. A more detailed description of the convection cell can be found in 105.

The Prandtl number $\operatorname{Pr}=\frac{\nu}{\kappa}$, Reynolds number $R e=\frac{U \cdot H}{\nu}$, Rayleigh number $R a=$ $\frac{g \cdot \beta \cdot \Delta T \cdot H^{3}}{\nu \cdot \kappa}$ and Archimedes number $A r=\frac{R a}{R e^{2} \cdot P r}$, are based on the fluid properties of air (kinematic viscosity $\nu$, thermal diffusivity $\kappa$ and isobaric thermal expansion coefficient $\beta$ ), the gravitational acceleration $g$, the mean inlet velocity $U$, the height of the sample $H$ and the temperature difference between bottom and top of the sample $\Delta T$.

The measurement plane of the combined PIV and PIT measurement is located horizontally in the $x-y$ plane at $z=0.03 \times H$, as close to the heated bottom plate as possible, see Figure 8.1. It should be noted, that the field of view (black rectangle in Figure 8.1) with the extension of approximately $150 \mathrm{~mm} \times 200 \mathrm{~mm}$, covers a rather small fraction of the horizontal cross section of the sample $(2500 \mathrm{~mm} \times 500 \mathrm{~mm})$. The spatial resolution of the velocity and temperature fields amounts to approximately $2 \mathrm{~mm}$. The time resolution of this measurement, i.e. the time lag between two recordings, amounts to $0.25 \mathrm{~s}$. This is less than one tenth of the turnaround times of the forced convection (FC) and TC induced LSCs, 


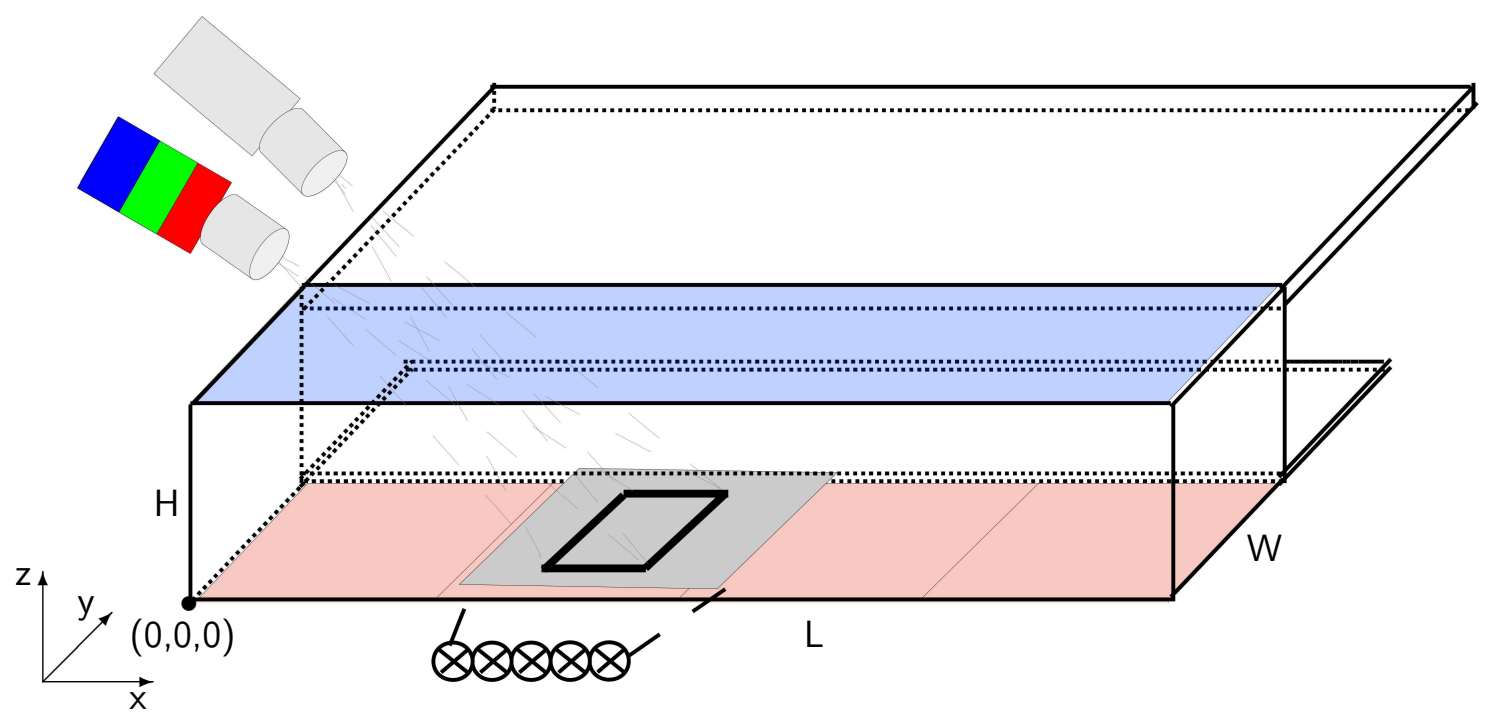

Figure 8.1: Sketch of the convection sample. The bottom plate of the cell is heated (red) while the ceiling is kept at ambient temperature (blue). The in- and outflow channels are located at the same side wall and span the whole length of the cell. The illuminated area is marked in grey whereas the field of view is shown as black rectangle.

see 98 . Hence, for each turnaround of the LSCs at least ten velocity and temperature fields are recorded. A measurement series comprises 700 images, covering up to 70 turnarounds of the LSCs.

To classify the height of the measurement plane above the heating plate in terms of other relevant distances in the convection sample, the thickness of the bottom thermal boundary layer $\delta_{T}$ is estimated as follows. From pure Rayleigh Bénard convection, one knows that $\delta_{T}=H / 2 N u$, with the height of the sample $H$ and the Nusselt number $N u=\frac{\Phi_{q} \cdot H}{k \cdot \Delta T}$, see for instance [18, 137]. Where $\Phi_{q}$ denotes the spatially and time-averaged heat flux per unit area and $k$ the thermal conductivity of the fluid. Using the prefactors of Grossmann and Lohse [48] (or newer works, e.g. [122]), we estimate $\delta_{T} \approx 7.6-5.4 \mathrm{~mm}$ for our investigated $R a=1.0-3.2 \times 10^{8}$ regime and $\operatorname{Pr}=0.71$. This value can be seen as upper boundary for $\delta_{T}$ in our mixed convection experiments as well, since the additional shear will only decrease $\delta_{T}$. Hence, our measurement plane at $Z=15 \mathrm{~mm}$ is located close above the bottom thermal boundary layer for all investigated $R a$. However, it should be noted, that Wagner et al. [137] showed that the thickness of $\delta_{T}$ can locally increase by a factor of 2 compared to $H / 2 N u$. Consequently, locally and temporally, the thermal boundary layer can reach into our measurement plane.

\subsubsection{CAlibration}

The calibration is conducted in the same way as presented in 101. However, it must be noted, that during the temperature - hue calibration of the TLCs in this setup an aggravation occurred. Occasionally, calibration values are only available in an intermediate 
hue regime. An explanation for the limitation of the hue-temperature calibration is that the used thermistor caused reflections in the hue field in a larger area than it did in the calibration measurement conducted in [101]. Hence, the hue evaluation position could not be chosen as close to the thermistor as before. This results in the need of spatial filtering of the hue values over four positions, yielding the absence of very small and very high hue values for the calibration. As a result we could determine a hue-temperature dependency as depicted in Figure 8.2 .

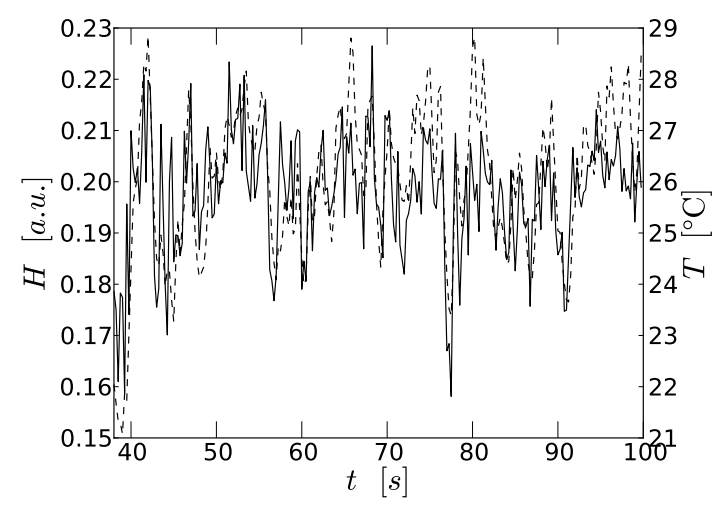

(a)

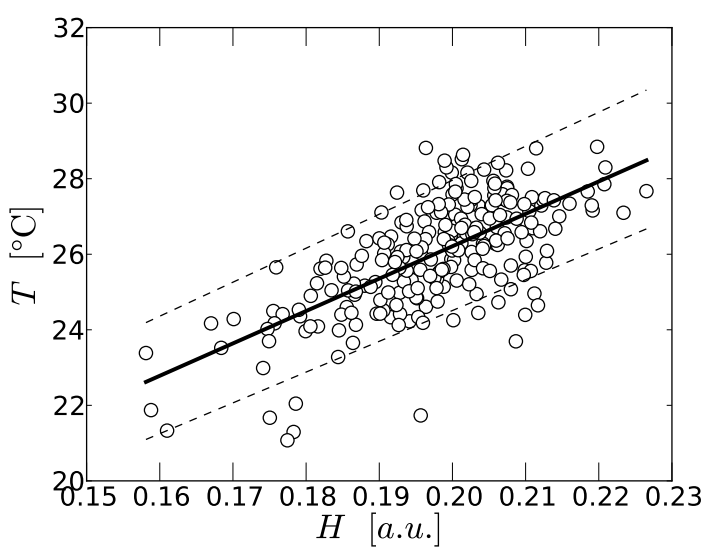

(b)

Figure 8.2: Hue-temperature calibration of the TLCs: (a) Time series of the hue (-, left axis) and temperature signals (--, right axis). (b) Temperature as a function of hue with a linear fit including its uncertainty based on the standard deviation.

It is well known, that TLCs are characterised, in general, by non-linear calibration functions. However, we decided to use a linear one, since the mean error of the fitted curve turned out to be only marginally larger than that of a fourth order fit. Furthermore, the temperature range of the calibration measurements does not cover the full range of measured hue values during the measurement campaign. Hence, a linear calibration function offers smaller uncertainty for the extrapolation. The fitted curve has the function $T\left[{ }^{\circ} \mathrm{C}\right]=b+m \cdot H$. The fitting parameters amount to $b=9.1(1.2)$ and $m=85.8(6.1)$, while the variance of residuals (reduced chisquare) of the fitted curve amounts to $\sigma_{T}=1.2 \mathrm{~K}$.

A detailed sensitivity analysis and a discussion of the measurement accuracy is in given in [101]. As opposed to the measurement therein, we used different types of TLCs, here R20C20W CLCR Hallcrest, to capture a larger temperature range. This results in a reduced absolute temperature accuracy at a similar dynamic range of the calibration.

\subsection{Results AND Discussion}

Sheet-like plumes can be observed when looking from above into the thermal boundary layer. This should be also true for the fingerprints of the sheet-like plumes when the measurement plane is located close above the thermal boundary layer. Herein, the formation of swirls 
should also be visible. However, with increasing distance of the measurement plane to the thermal boundary layer, turbulent interactions with the bulk will more and more fragment the sheet-like plumes (see [18]). Hence, an increase of this distance will raise the effect of the bulk interactions. Thus, the fraction of the undisturbed line plumes will decrease.

It must be noted that a measurement within a layer requires special attention, since only two velocity components are recorded in a two dimensional field of view. However, the fluid motion and hence also the path of the plumes can not be expected to be two dimensional at these $R a$. Therefore, in general, there will be a non negligible velocity component perpendicular to the measurement plane, which is not captured by the applied measurement technique.

The investigated $R a-R e-A r$ parameter combinations are summarised in table 8.1. Herein, also the mean values of $\langle u\rangle_{x, y, t},\langle v\rangle_{x, y, t},\langle h\rangle_{x, y, t}$ and $\langle T\rangle_{x, y, t}$ averaged in space and time, after an outlier detection was applied, are given. They will be discussed later.

TABLE 8.1: Parameter range as well as time and space averaged values. The standard deviations are given in parentheses.

\begin{tabular}{cccccccc}
\hline case & $\begin{array}{c}R a \\
{\left[\times 10^{8}\right]}\end{array}$ & $\begin{array}{c}R e \\
{\left[\times 10^{4}\right]}\end{array}$ & $\begin{array}{c}A r \\
{[1]}\end{array}$ & $\begin{array}{c}\langle u\rangle_{x, y, t} \\
{\left[\mathrm{~ms}^{-1}\right]}\end{array}$ & $\begin{array}{c}\langle v\rangle_{x, y, t} \\
{\left[\mathrm{~ms}^{-1}\right]}\end{array}$ & $\begin{array}{c}\langle h\rangle_{x, y, t} \\
{[a . u .]}\end{array}$ & $\begin{array}{c}\langle T\rangle_{x, y, t} \\
{\left[{ }^{\circ} \mathrm{C}\right]}\end{array}$ \\
\hline $\mathrm{A} 01$ & 1.0 & 0.5 & 5.3 & $0.025(8)$ & $-0.024(10)$ & $0.166(3)$ & $23.3(1.6)$ \\
$\mathrm{A} 03$ & 1.4 & 0.5 & 7.6 & $0.012(17)$ & $-0.095(11)$ & $0.177(2)$ & $24.2(1.7)$ \\
$\mathrm{A} 05$ & 1.3 & 1.0 & 1.8 & $-0.054(15)$ & $0.097(10)$ & $0.172(2)$ & $23.8(1.6)$ \\
$\mathrm{A} 07$ & 1.7 & 1.0 & 2.4 & $-0.097(19)$ & $0.082(15)$ & $0.177(3)$ & $24.2(1.7)$ \\
$\mathrm{A} 08$ & 1.7 & 0.8 & 3.5 & $0.025(15)$ & $-0.103(12)$ & $0.186(2)$ & $25.0(1.7)$ \\
$\mathrm{A} 09$ & 2.3 & 1.0 & 3.1 & $0.026(25)$ & $-0.088(28)$ & $0.181(6)$ & $24.6(1.8)$ \\
$\mathrm{A} 11$ & 2.2 & 1.7 & 1.1 & $-0.064(16)$ & $0.150(13)$ & $0.168(4)$ & $23.5(1.7)$ \\
$\mathrm{A} 12$ & 2.6 & 1.2 & 2.7 & $0.132(26)$ & $0.044(26)$ & $0.179(4)$ & $24.4(1.7)$ \\
$\mathrm{A} 13$ & 3.2 & 1.2 & 3.3 & $0.030(29)$ & $-0.096(37)$ & $0.192(7)$ & $25.5(1.8)$ \\
\hline
\end{tabular}

This section is outlined as follows: first, we will present series of instantaneous velocity and temperature fields to visualise the flow field within the investigated layer. Afterwards, probability density functions (PDFs) of the temperature are presented to analyse how the relevance of plumes compared to the bulk depends on the characteristic numbers. This is followed by a section on the convective heat transport. Finally, mean flow fields, the standard deviation of the temperature and the $A r$ dependency of the mean angle of the velocity within the measurement plane are shown and discussed.

\subsubsection{VisUALISATION OF THE DYNAMICS}

Although the measurements are performed above the thermal boundary layer, which has a thickness of only a few millimetres (always less than $10 \mathrm{~mm}$ ), still the thermal fingerprints of the so called sheet-like or line plumes [83, 108], indicated by the red structures in Figures 
8.3 8.5, are clearly visible. The images are recorded at a frequency of $4 \mathrm{~Hz}$ being high enough to study the plume dynamics. All depicted cases are recorded at approximately constant $R e=1.0-1.2 \times 10^{4}$.

In Figure 8.3 subsequent instantaneous velocity and temperature fields are shown for case A05. Few temperature fingerprints of the sheet-like plumes and their predominant orientation parallel to the local shear are visible. Furthermore, the lower values of both velocity components, especially of the $v$ component, in the region around $y=50 \mathrm{~mm}$, indicates that the mean wind of the large-scale circulation crosses the measurement plane here. With increasing $y$, the $v$ component increases as well. The temperature range spans values from 21.6 to $26.8^{\circ} \mathrm{C}$. A detailed analysis of the distribution of the temperature in terms of PDFs is given in the next section.

Instantaneous fields recorded for case A09 are visualised in Figure 8.4. In comparison to the previously discussed Figure 8.3, $R a$ is increased from $R a=1.3 \times 10^{8}$ to $R a=2.3 \times 10^{8}$. Accordingly, $A r$ changes from $A r=1.8$ to $A r=3.1$. The first to be mentioned is the higher dynamic range of the temperature, i.e. the temperature values span from 21.6 to $28.0^{\circ} \mathrm{C}$, which is an increase of $25 \%$ compared to the previously discussed parameter combination. Furthermore, the prevalent flow direction is directed in negative $y$ direction. This means, that the large-scale circulation rotates in the opposite direction than in case A05. This so called "bypass" flow was also found for high $A r$ in earlier investigations, see [98]. Furthermore, the fingerprints of the sheet-like plumes in Figure 8.4 are orientated not necessarily parallel to the local shear, i.e. the local velocity. A possible explanation is that the thickness of the thermal boundary layer $\delta_{T}$ decreased compared to case A05. From pure Rayleigh Bénard convection, one knows that $\delta_{T}=H / 2 N u$ scales with $R a^{-0.285} 137$. Hence, for mixed convection at a constant $R e$, we expect a similar behaviour. This means that the lower $\delta_{T}$ and the constant position of the measurement plane leads to a increasing distance of the measurement plane relative to $\delta_{T}$, i.e. a measurement position further away from the boundary layer. According to Bosbach et al. [18], the interactions of the sheet-like plumes with the turbulent fluctuations of the temperature and/or the velocity in the bulk lead to a fragmentation of the plumes. Since an increase of $R a$ implies stronger fluctuations, the sheet-likes plumes are more fragmented than the former ones in case A05.

In the bulk, fingerprints of thermal plumes are not expected to be aligned with the flow velocity. Thermal structures, which were sheet-like plumes within the thermal boundary layer spiral up and become the stems of the mushroom like plumes with increasing distance from the boundary layer. For the investigated parameters, the measurement plane seems to be located within this mixing region, since both types of structures, i.e. sheet-like plumes and larger agglomerated regions reflecting the stems of the mushroom like plumes, are found.

In case A13, presented in Figure 8.5. $R a$ is increased from $R a=2.3 \times 10^{8}$ to $R a=3.2 \times 10^{8}$ at constant $A r \approx 3.2$, in comparison to the previously discussed case A09. In case A13 the temperature range spans from 21.6 to $27.6{ }^{\circ} \mathrm{C}$, see Figure 8.5 , which is a slightly smaller range than the ones in $\mathrm{A} 05$ and $\mathrm{A} 09$. It is noteworthy that we find lower temperatures at a 


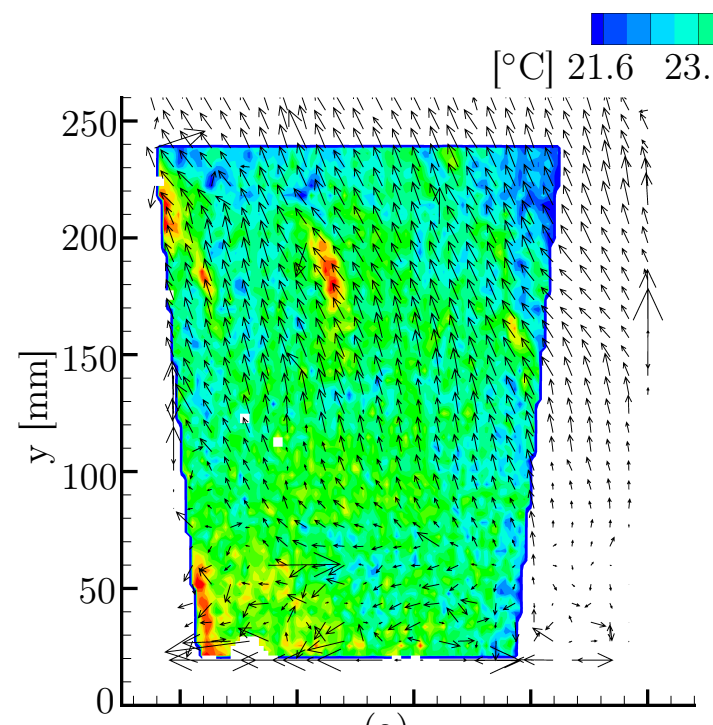

(a)

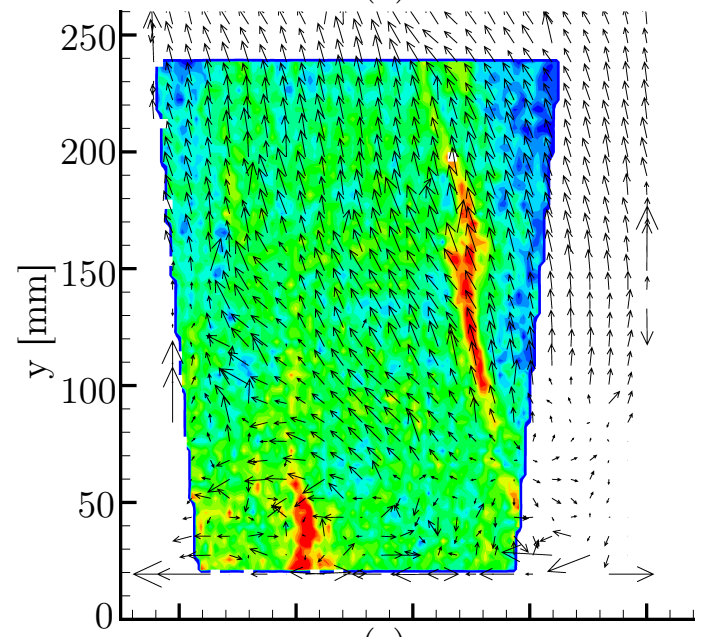

(c)

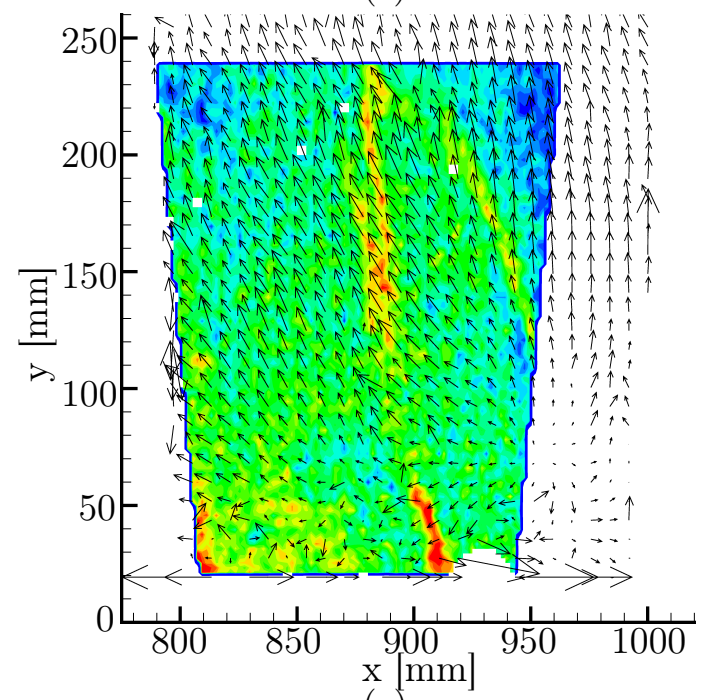

(e)
$0.2 \mathrm{~m} / \mathrm{s}$

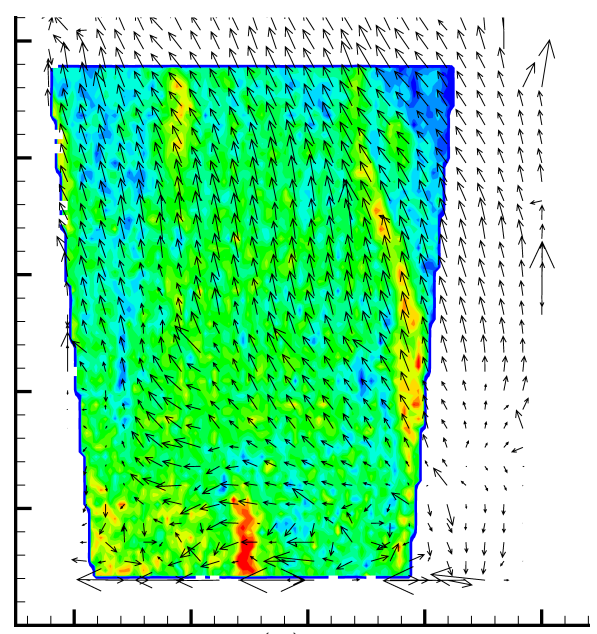

(b)

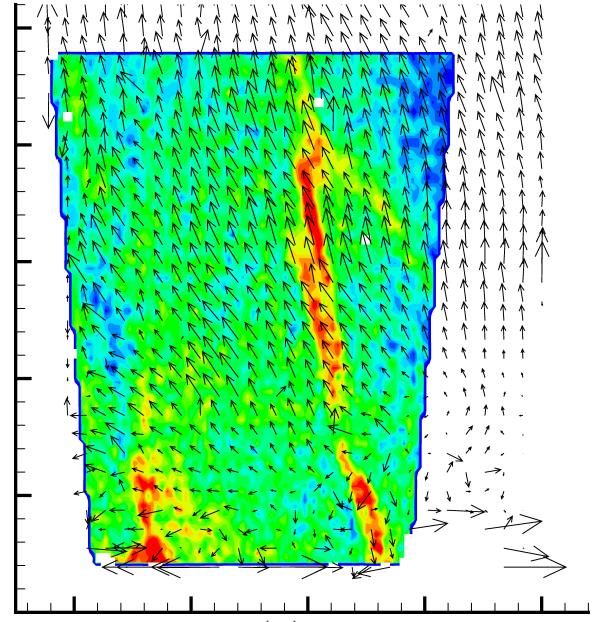

(d)

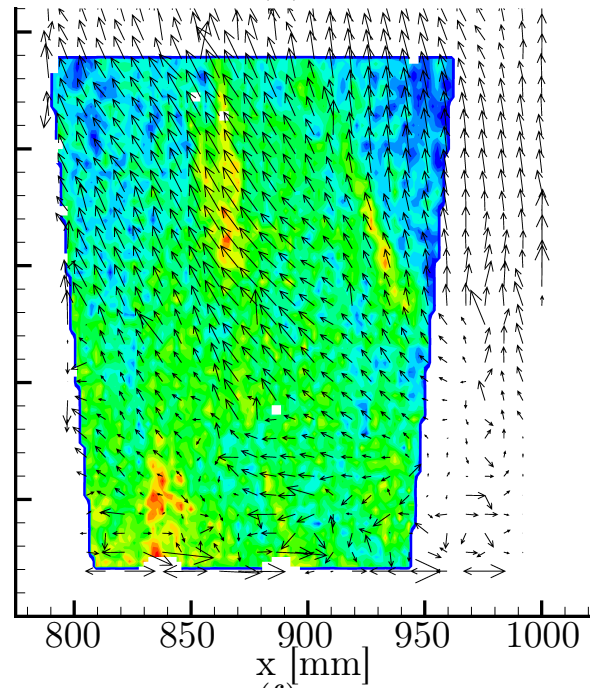

(f)

Figure 8.3: $R a=1.3 \times 10^{8}, R e=1.0 \times 10^{4}$ and $A r=1.8$ (A05): Instantaneous temperature contours and velocity vectors. Images are recorded at $4 \mathrm{~Hz}$. The legend at the top applies to all images. 


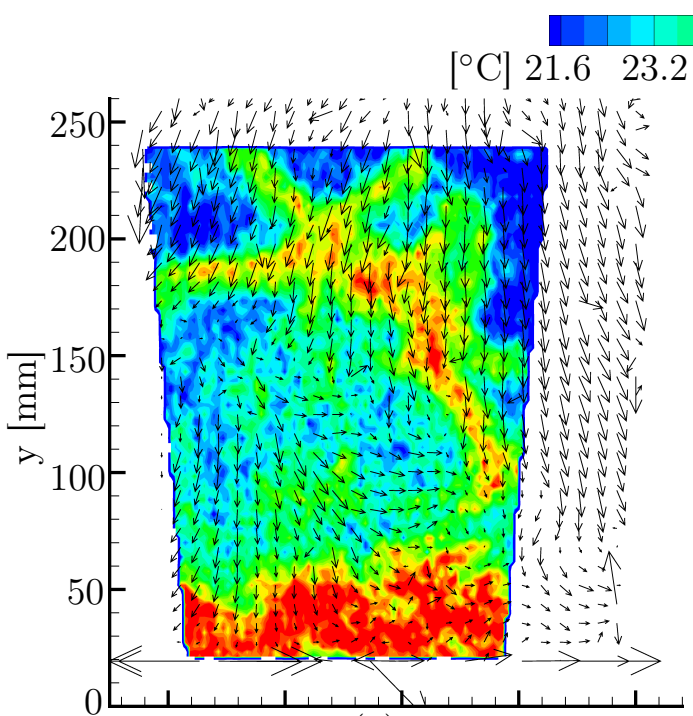

(a)
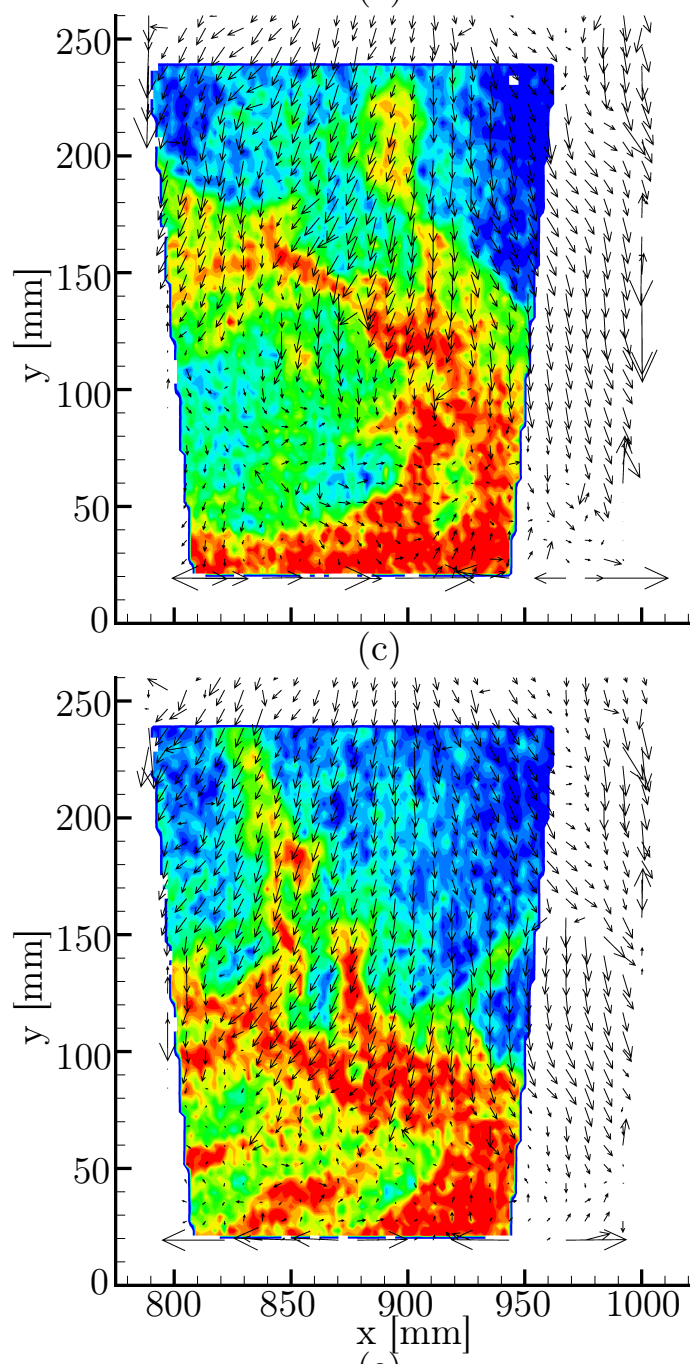

(e)

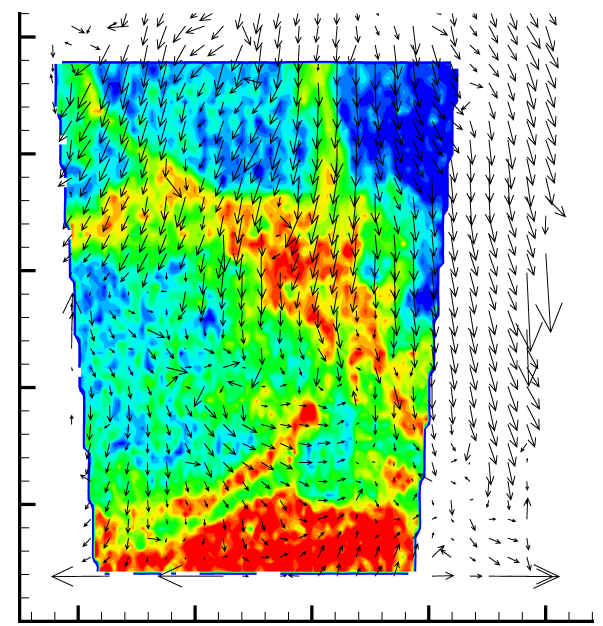

(b)

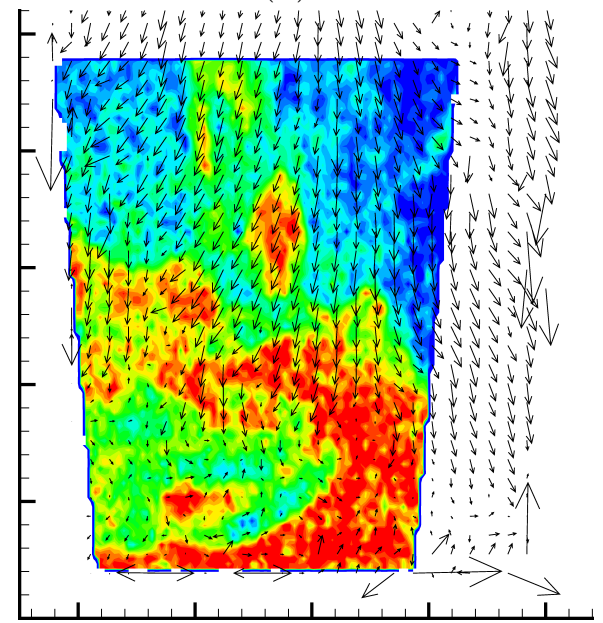

(d)

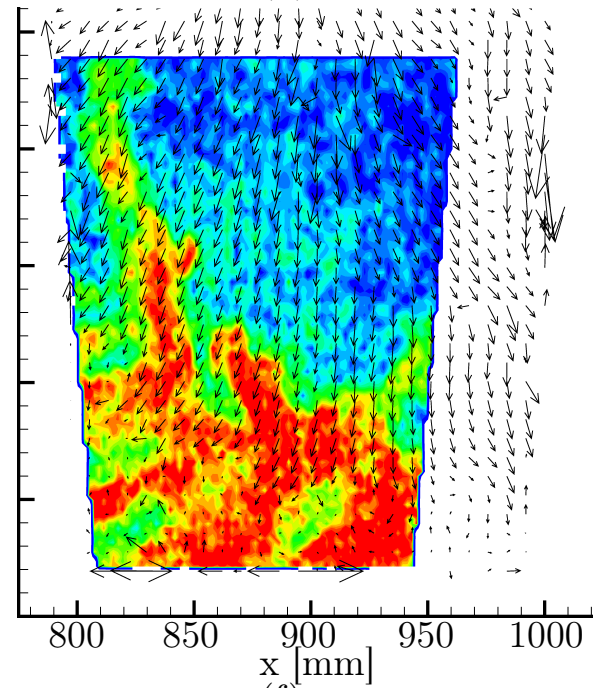

(f)

Figure 8.4: $R a=2.3 \times 10^{8}, R e=1.0 \times 10^{4}$ and $A r=3.1$ (A09): Instantaneous temperature contours and velocity vectors. Images are recorded at $4 \mathrm{~Hz}$. The legend at the top applies to all images. 


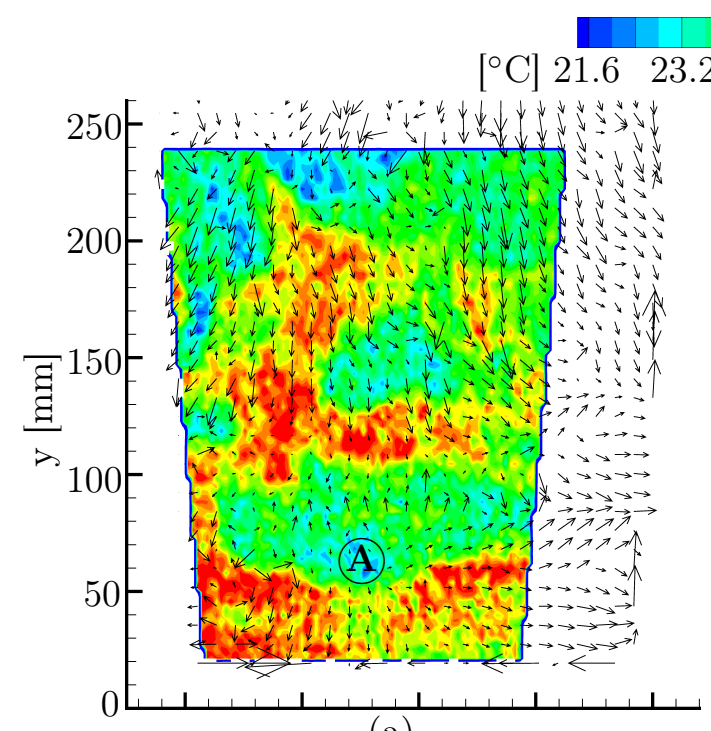

(a)

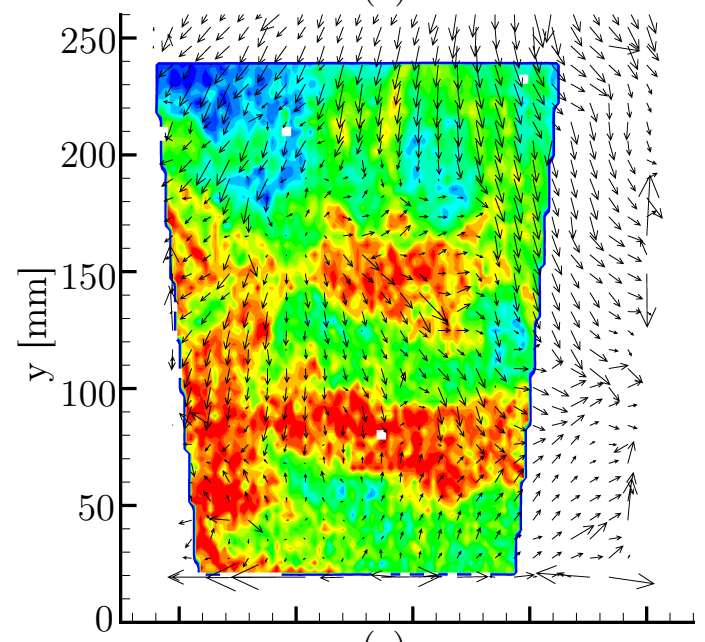

(c)

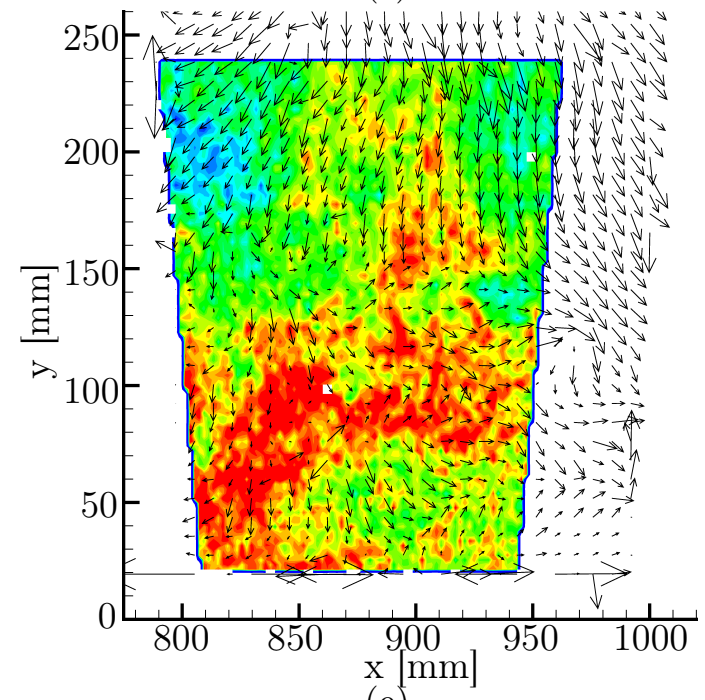

(e)

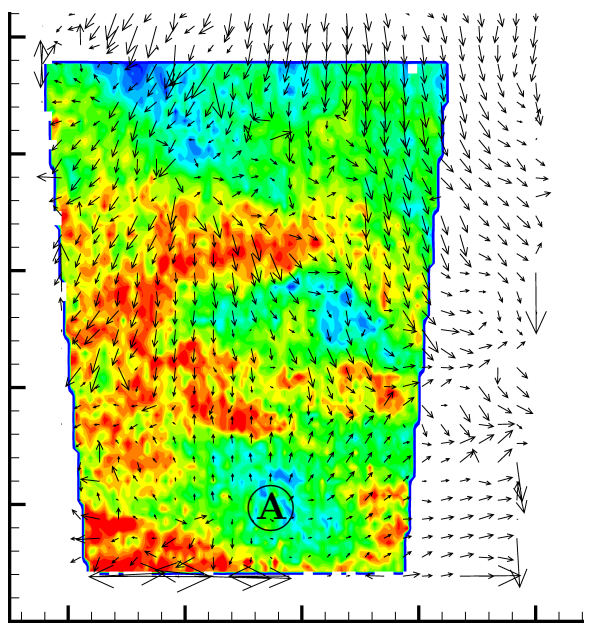

(b)

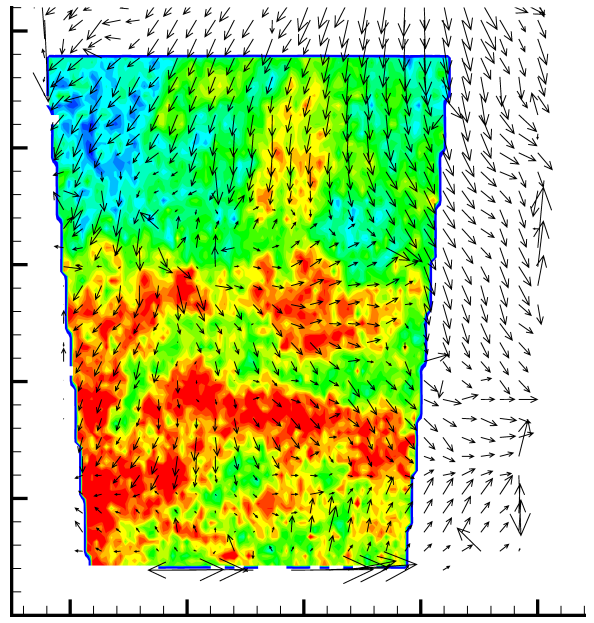

(d)

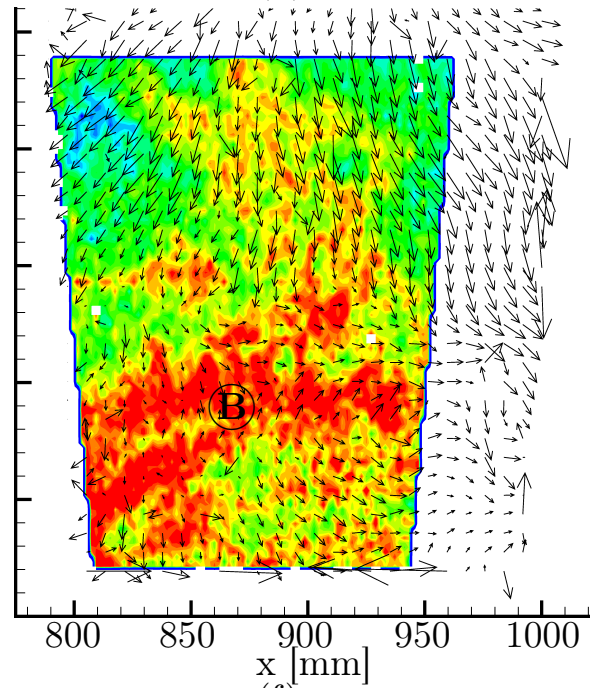

(f)

Figure 8.5: $R a=3.2 \times 10^{8}, R e=1.2 \times 10^{4}$ and $A r=3.3$ (A13): Instantaneous temperature contours and velocity vectors. Images are recorded at $4 \mathrm{~Hz}$. The legend at the top applies to all images. 
higher $R a$, i.e. a higher temperature of the bottom plate of our sample. However, the mean values averaged in space and time, see table 8.1, reveal that the lower temperatures at the higher $R a$ can only be a temporally event, since the mean value $\langle T\rangle_{x, y, t}$ is higher in A13 than for A09. This is confirmed by the time evolution of the spatially averaged temperatures in Figure 8.6 (c), which is characterised by time periods in which the temperature of A13 is lower than for A09. Looking again at Figure 8.5, no sheet-like plume structures are visible anymore. From this we conclude that stronger bulk fluctuations interact with the plumes for higher $R a$. Hence, the fragmentation of the sheet-like plumes further increased and these plumes are not visible in the recorded instantaneous fields anymore. However, the dynamics and the degree of the three dimensionality of the flow field is higher. To illustrate the lower homogeneity of the velocity field, "source" and "sink" regions are marked as A and $\mathrm{B}$ in Figure 8.5. For example, (a) and (b) reflect the source region " $\mathrm{A}$ " in the velocity field. It can be explained by downwelling of cold fluid in this region. Hence, on this $2 \mathrm{C}-2 \mathrm{D}$ vector map the source region is well correlated with a region of lower temperature. In contrast, in Figure 8.5 (f), the sink region "B" comprises rising warm air. Accordingly, there the stem of a plume is formed and this sink region is characterised by an elevated temperature. In the intermediate figures, the breakdown and formation of these regions is reflected.

To analyse the flow dynamics on longer time scales, we present the time evolution of the velocity components and the temperatures recorded on a time scale of up to three minutes. In addition to parameter combinations A05, A09 and A13, which are discussed above, results obtained for A11 are included. First, it must be noted that all three quantities, see Figures 8.6 (a) to (c) behave similar for A05 and A11 as well as for A09 and A13. Since for each of these two pairs $A r$ is of similar magnitude, whereas $R a$ and $R e$ differ significantly, the former seems to be the liable characteristic number. Moreover, the similar $R a$ realised in A09 and A11 or the similar Re in A05 and A09 lead not to similar the flow fields.

Surprisingly, Figure 8.6 (a) reveals, that, depending on $A r$, the spatially averaged velocity component in $x$ direction $\langle u\rangle_{x, y}$ are either positive for high $A r$ or negative for low $A r$ during the complete time period.

Furthermore, the cases A09 and A13 reveal much stronger fluctuations of $\langle u\rangle_{x, y}$. Additionally, the spatially averaged velocity component $\langle v\rangle_{x, y}$, shown in Figure 8.6 (b) indicates that the main flow in $y$-direction depends on $A r$ as well. This finding is in good agreement with the earlier observed "bypass" flow, which occurs above certain $A r$ [98]. For $A r$ below this threshold, the inflowing air follows the ceiling towards the front of the sample before it descends and proceeds in positive $y$-direction above the heating plate. Above this threshold, the thermally induced LSCs in the sample are so dominant, that the rather cold inflowing air is downwelling directly after entering the sample. Hence, it descends in the rear and separates close to the air outlet. Accordingly, the main flow above the heating plate is directed in negative $y$-direction. Higher values of $\langle v\rangle_{x, y}$ for A11 than for A05 correlate with the higher Re. Surprisingly, for A09 and A13 the values of $\langle v\rangle_{x, y}$ are similar, although $R e$ differs by $20 \%$. From this we conclude that for $A r \gtrsim 3, R e$ does not directly determine the strength of the flow in $y$ direction. Finally, higher fluctuations are observed again for A09 


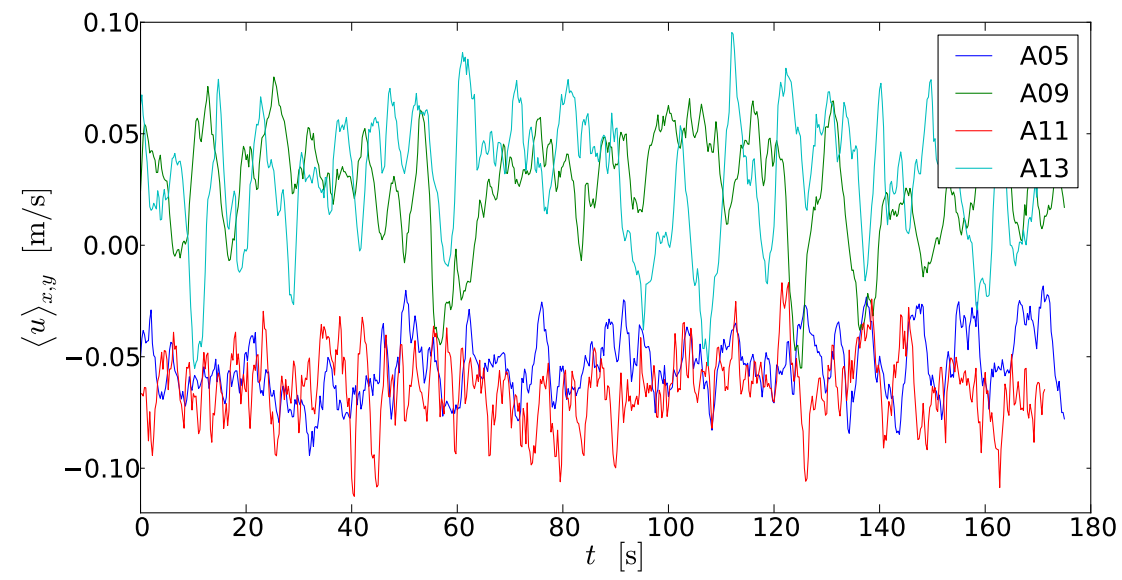

(a)

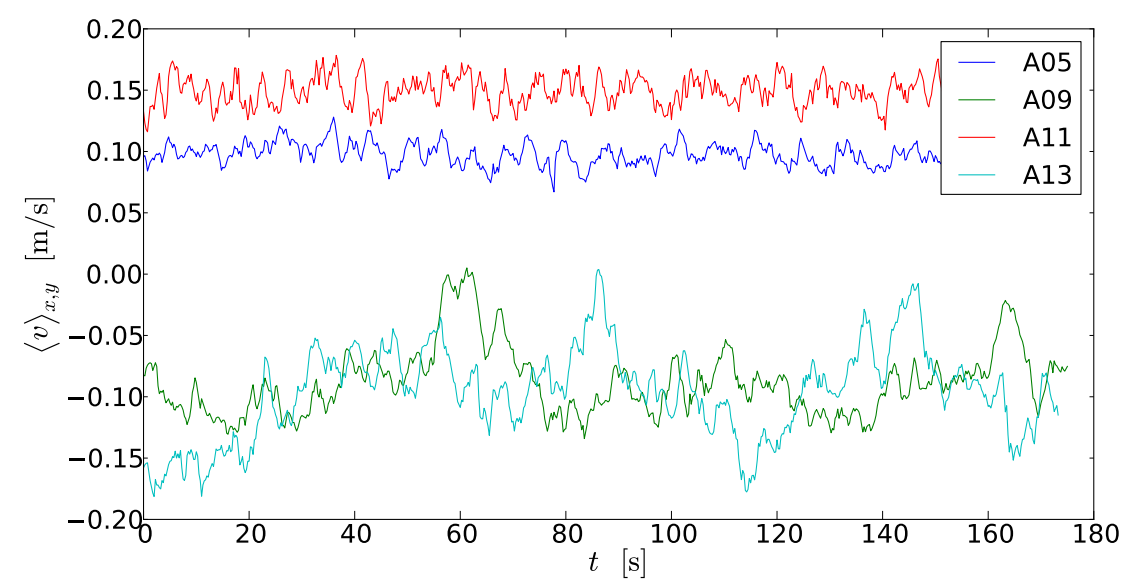

(b)

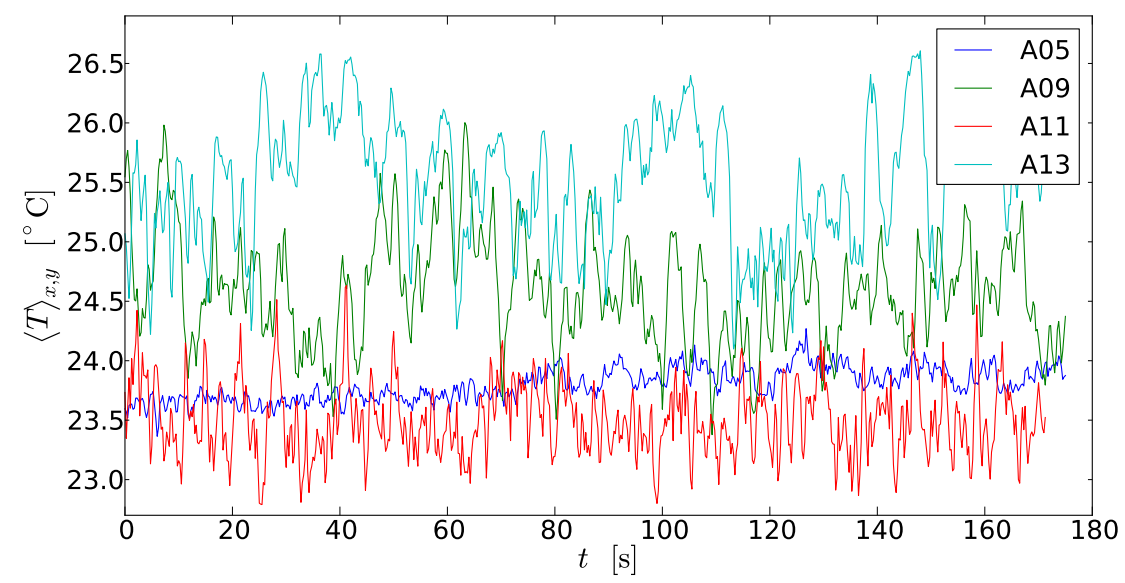

(c)

FiguRE 8.6: Time evolution of spatially averaged velocity components and temperature: (a) $\langle u\rangle_{x, y}$, (b) $\langle v\rangle_{x, y}$ and (c) $\langle T\rangle_{x, y}$. For A05 (-), A09 (-), A11 (-) and A13 (-). 
and A13 than for A05 and A11

Finally, the spatially averaged temperature $\langle T\rangle_{x, y}$ shown in Figure 8.6 (c) reveal that the mean temperatures increase with rising $A r$, i.e. in the order A11, A05, A09 and A13. However, the temperature fluctuations for A11 are significantly higher than for A05. This indicates that $R a$ rather than $A r$ determines the strength of the temperature fluctuations. A more detailed analysis of the temperature dependency on $R a$ and $A r$ is given in the following section.

\subsubsection{Plume DYNAMics AND SCAling}

To analyse the influence of thermal plumes on the temperature distribution, probability density functions (PDFs) of the temperature are evaluated. Below they are discussed based on histogram plots of all measured temperatures within the measurement plane and for all time steps. In total approximately $5.5 \times 10^{6}$ measurement points were taken into account for each case. For a quantitative analysis, a sum $F(T)$ of two Gaussian curves $G 1(T)$ and $G 2(T)$ is fitted to each histogram. The Gaussian curves are defined by $G_{i}(T)=\Psi_{i} \cdot \exp \left\{-\frac{\left(T-\Theta_{i}\right)^{2}}{2 \cdot \sigma_{i}^{2}}\right\}$. Here, the fitting parameters $\Psi_{i}, \Theta_{i}$ and $\sigma_{i}$ denote the amplitude, the position and the width of the Gaussian curve, respectively. Results obtained for the above discussed four selected cases are depicted in Figure 8.7. After an interpretation of the histogram plots, the fit parameters are analysed.

The idea of summing up the two Gaussian curves is to ascribe the measured temperatures to different regimes. The first Gaussian curve comprises the mean fluid temperature within the measurement layer. There, assuming a Gaussian temperature distribution is justified, since the turbulent fluctuations of the temperature in the bulk of the sample can be assumed to behave like homogeneous isotropic turbulence, and thus, should be normal distributed [8]. However, the obtained PDFs show non-Gaussian tails, which can be fitted using a second Gaussian curve. The sum of these two Gaussian curves describes the PDFs with errors smaller than $5 \%$ for all fit parameters. We ascribe the second Gaussian curve to the thermal plumes, which pass the measurement plane on their way towards the bulk and the cold ceiling of the sample. Based on thermal dissipation rate PDFs, [60 presented a similar classification. Different parts of the PDFs were ascribed to the characterising regions in the sample: the bulk flow, the plumes/mixing layer and the conductive sublayers. Such a detailed classification is not possible with our measured data since it is obtained in the rather small field of view.

Nonetheless, Figure 8.7 presents four PDFs of the temperature for the above discussed parameter combinations. Recall, that $R e$ is the same for A05 (Figure 8.7, a) and A09 (Figure 8.7. c)). The strong influence of increasing $R a$ and hence rising $A r$ on the temperature fluctuations is reflected by the growth of the second Gaussian peak. Moreover, comparing the PDFs of A09 with A11 (Figure 8.7, b) the rise of $A r$, i.e. decrease Re, results again in a strong accumulation of the second Gaussian peak. Finally, a comparison of A09 with A13 (Figure 8.7, d) reveals the influence of rising $R a$ and $R e$. Again an increase of the second 

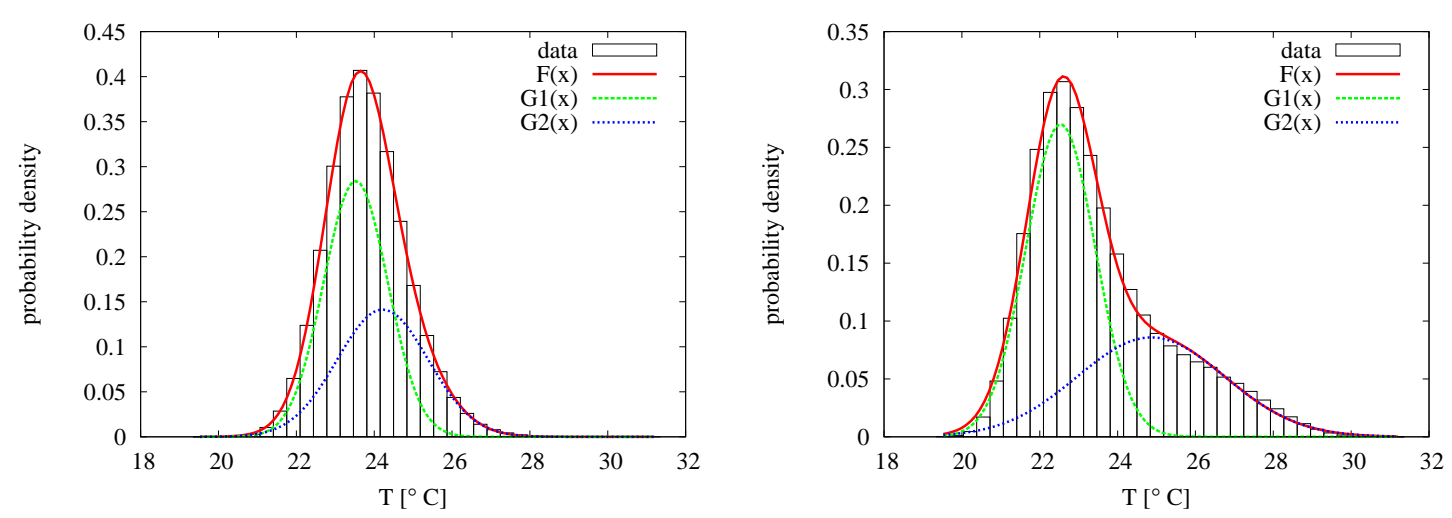

(a) A05: $R a=1.3 \times 10^{8}, R e=1.0 \times 10^{4}, A r=1.8$

(b) A11: $R a=2.2 \times 10^{8}, \operatorname{Re}=1.7 \times 10^{4}, A r=1.1$
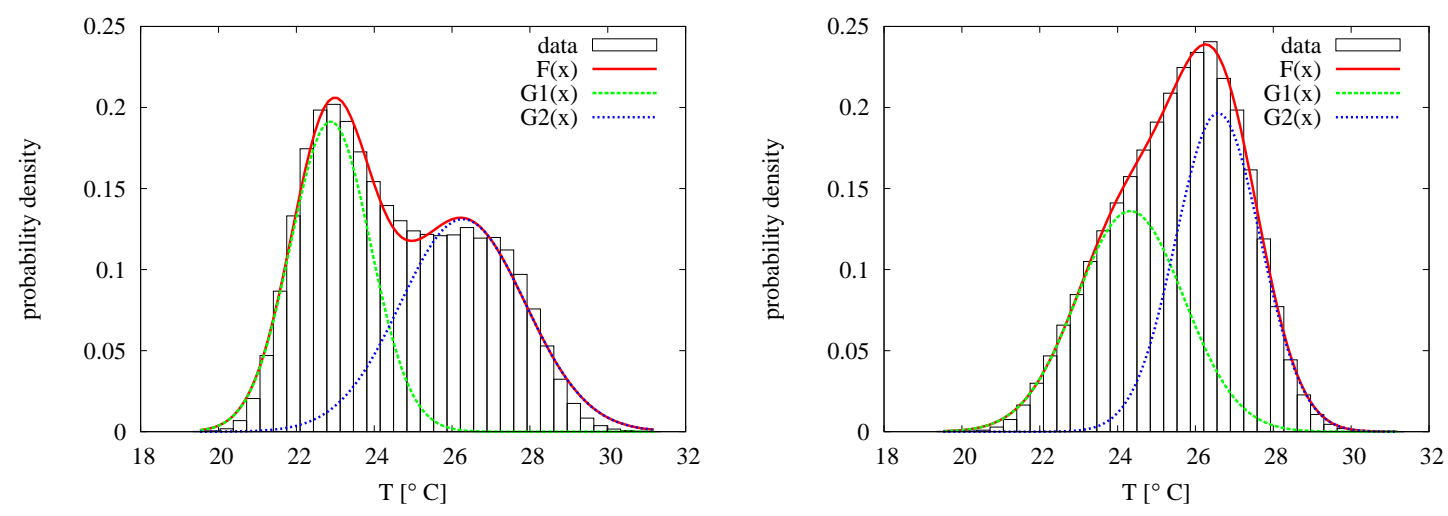

(c) A09: $R a=2.3 \times 10^{8}, R e=1.0 \times 10^{4}, A r=3.1$

(d) A13: $R a=3.2 \times 10^{8}, R e=1.2 \times 10^{4}, A r=3.3$

Figure 8.7: PDFs of the temperature fields. A sum of two Gaussian functions is used for the fitting.

Gaussian peak as compared to the first one is found. Consequently, the second Gaussian curve gains importance with rising $R a$ and $A r$ and decreasing $R e$. A simple dependency on a single characteristic number only could not be found.

Furthermore, comparing the PDF of A13 with those of A09 and A11, reveals a more homogeneous temperature distribution. The reason might be that less sheet-like plumes are located in the measurement plane since the thickness of the thermal boundary layer decreases with increasing $R a$. According to Bosbach et al. [18] the higher $R a$ leads to stronger velocity fluctuations in bulk, which fragment the sheet-like plumes. This process smears out the temperature fingerprint of the plume and thus, the temperature deviation between plume and ambient fluid decreases. Hence, in A13 the measurement plane covered a higher fraction of the bulk fluctuations, which are stronger for higher $R a$. However, in combination with smeared out temperature profiles of the sheet-like plumes, in total, a more homogeneous temperature distribution is measured as compared to lower distances to the thermal boundary layer where the plumes were not fragmented yet.

In the following, we analyse how the fitting parameters of the Gaussian curves depend on the characteristic numbers. Figures 8.8 (a) and (b) depict the ratios of the amplitudes of 


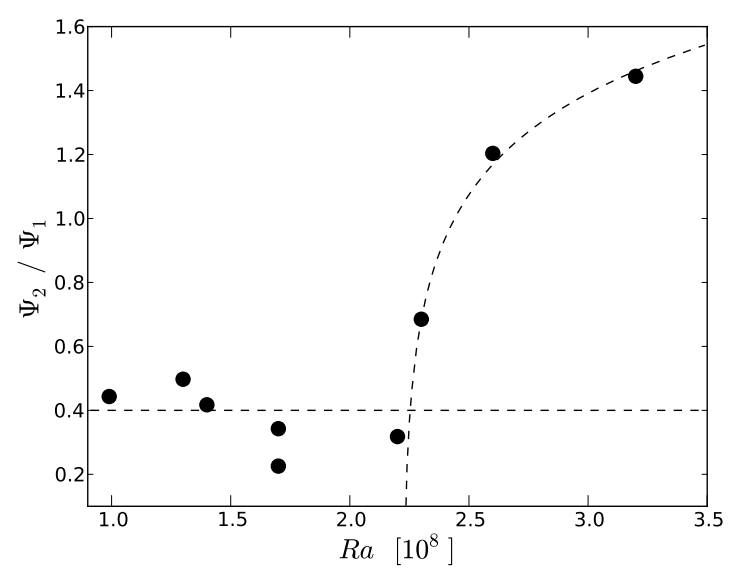

(a)

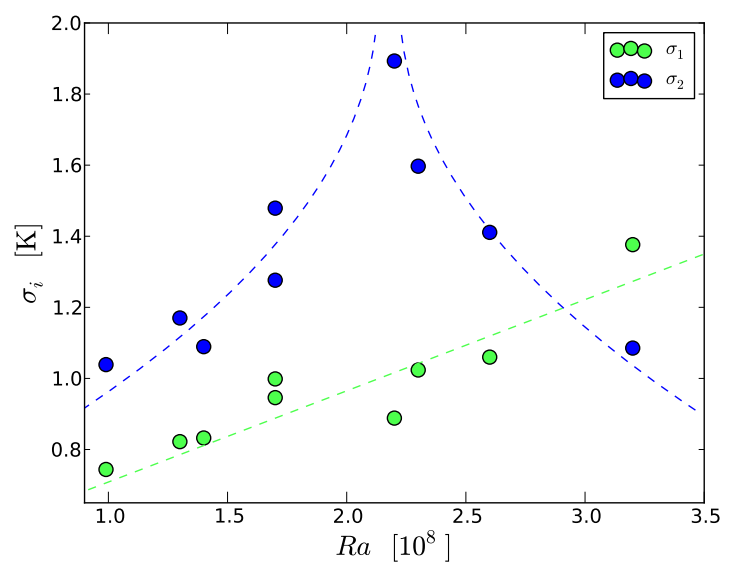

(c)

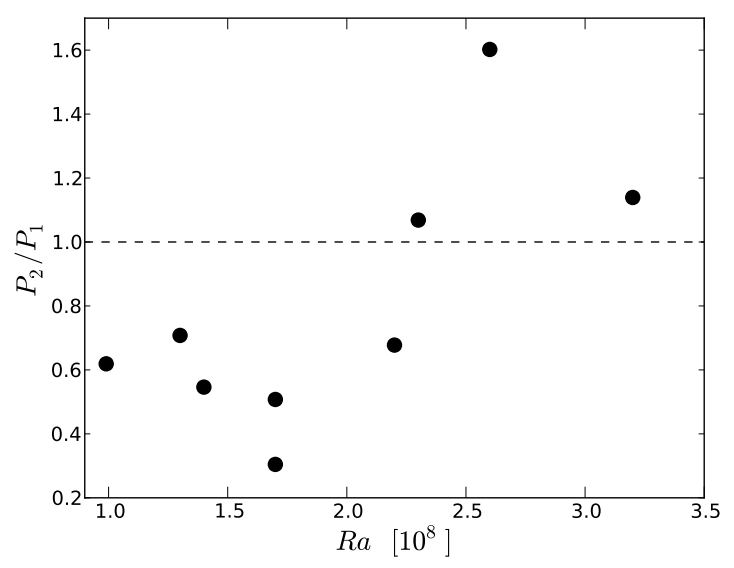

(e)

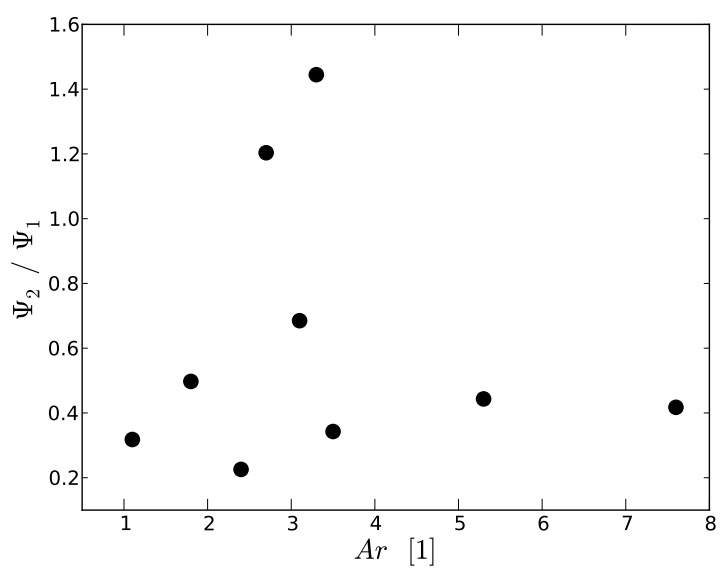

(b)

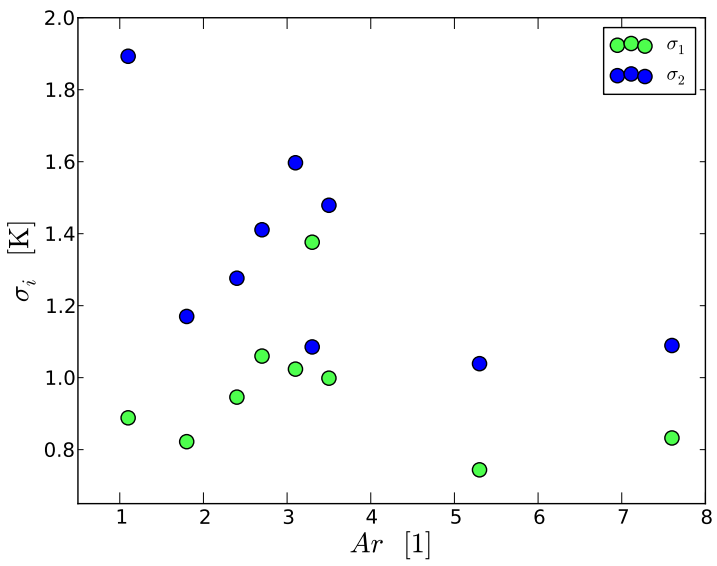

(d)

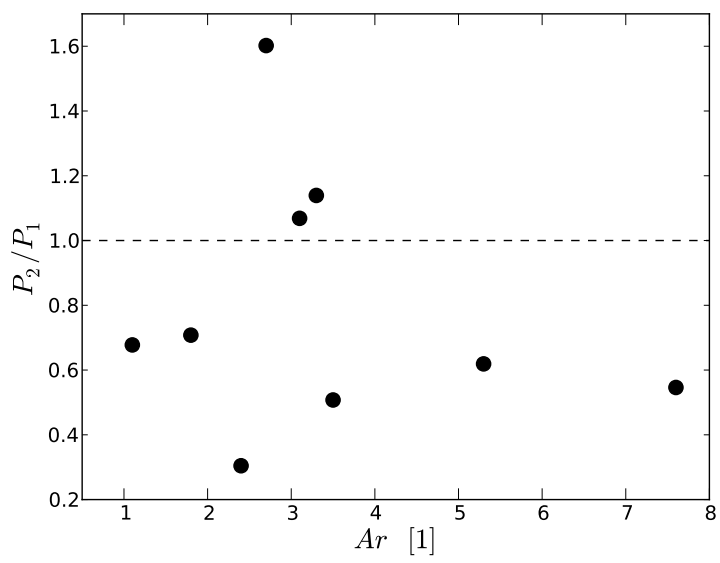

(f)

Figure 8.8: Analysis of the Gaussian curves. Ratio of the amplitudes $\Psi_{2} / \Psi_{1}$ (a) as a function of $R a$ and (b) as a function of $A r$. Width of both curves $\sigma_{i}$ (c) as a function of $R a$ and (d) as a function of $A r$. The plume fraction $P_{2} / P_{1}$, i.e. the ratio of the temperature abundances, (e) as a function of $R a$ and (f) as a function of $A r$. 
the two Gaussian curves as functions of $R a$ and $A r$, respectively. In Figure 8.8 (a) the lines are just guides to the eye and do not raise a claim to a physical interpretation. Roughly speaking, the ratio $\Psi_{2} / \Psi_{1}$ is constant up to a critical $R a_{c} \approx 2.25 \times 10^{8}$ and increases strongly for higher $R a$. Such a trend is not visible for $A r$ in Figure 8.8 (b). However, elevated values of $\Psi_{2} / \Psi_{1}$ are found only an intermediate $A r \approx 2.7-3.3$ regime. Further, in Figure 8.8 (c) the widths $\sigma_{i}$ of the Gaussian curves are plotted as a function of $R a$. They reveal two completely different trends. First, the width of the first curve scales linearly with $R a$. This behaviour suits the interpretation of the first Gaussian curve to reflect the mean fluid temperature in the absence of plumes, since turbulence for higher $R a$ is associated with larger temperature fluctuations. These higher fluctuations widen the first Gaussian curve. In contrast, the width of the second Gaussian curve $\sigma_{2}$ has a maximum at an intermediate $R a$. Here again, the lines are just guides to the eye. The position of the maximum is located at $R a_{c} \approx 2.2 \times 10^{8}$, well suiting the previously observed steep increase of $\Psi_{2} / \Psi_{1}$. Figure 8.8 (d) depicts $\sigma_{i}$ as a function of $A r$. Except for the width of the second curve at the lowest $A r$, both widths tend to a maximum at $A r \approx 3$. Since the widths are a measure for the strength of the fluctuations of the underlying temperatures, these maxima indicate that an intermediate $A r$ regime is essential for strong mixing processes within the measurement plane. Within the field of view the strongest interactions between the bulk and the plumes occur for these $A r$. The competition of buoyancy and inertia forces, leads to an instability in the range $A r \approx 2.7-3.3$, see Figures 8.8 (b), (d) and (f).

The existence of a critical $R a_{c}$ can be explained by looking at the relative distance of the measurement plane to the bottom thermal boundary layer. This was quantified in section 8.2.1. At low $R a$, only few strong plumes characterised by a sharp temperature distribution pass the measurement plane. At medium $R a$, the measurement plane is located within the mixing zone and the temperature distribution is smeared out. Finally, for higher $R a$, the measurement plane is located above the mixing zone, i.e. closer to the bulk of the sample leading to sharper temperature distributions around the mean fluid temperature in the bulk. This explanation is also in agreement with the findings from [18]. They ascribe the fragmentation process of plumes to interactions with wall domains of the LSCs and turbulent bulk fluctuations.

In order to analyse the plume fraction in the measurement plane, the ratios of the probabilities $P_{2} / P_{1}$ of the two Gaussian curves are plotted in Figure 8.8 (e) and (f) as a function of $R a$ and $A r$. The probabilities are defined as area under each single Gaussian curve: $P_{i}=\int_{-\infty}^{\infty} G_{i}(T) d T$. Hence, $P_{1}$ and $P_{2}$ reveal the abundance of temperatures which are ascribed to the bulk and the plumes, respectively. Accordingly, the ratio $P_{2} / P_{1}$ can be interpreted as plume fraction. The lines in the corresponding Figures 8.8 (e) and (f) are drawn at $P_{2} / P_{1}=1$. Hence, below the line the bulk temperatures and above the plumes temperatures are more abundant. According to Figure 8.8 (e) the transition point is located at $R a \approx 2.25 \times 10^{8}$, which is the same value discussed above. For $R a \lesssim 2.25 \times 10^{8}$ the bulk and for $R a \gtrsim 2.25 \times 10^{8}$ the plumes dominate the PDF. Finally, we depict the plume fraction as a function of $A r$, Figure 8.8 (f). Herein, we find that temperatures ascribed 
to the plumes are more abundant in an intermediate range at $2.7 \lesssim A r \lesssim 3.3$ than those which are bulk related. The importance of this intermediate $A r$ regime was underlined by Schmeling et al. [98], who performed a long-time analysis using temperature probes in the here investigated sample. Therein, unstable configurations of the LSC configurations were observed for $1.2 \lesssim A r \lesssim 2.6$. Whether the here observed change of the plume fraction is correlated to the observed oscillations and erratic reconfigurations can not be answered, since the measurement times in this study are too short to analyse the long-time processes studied in $[98$.

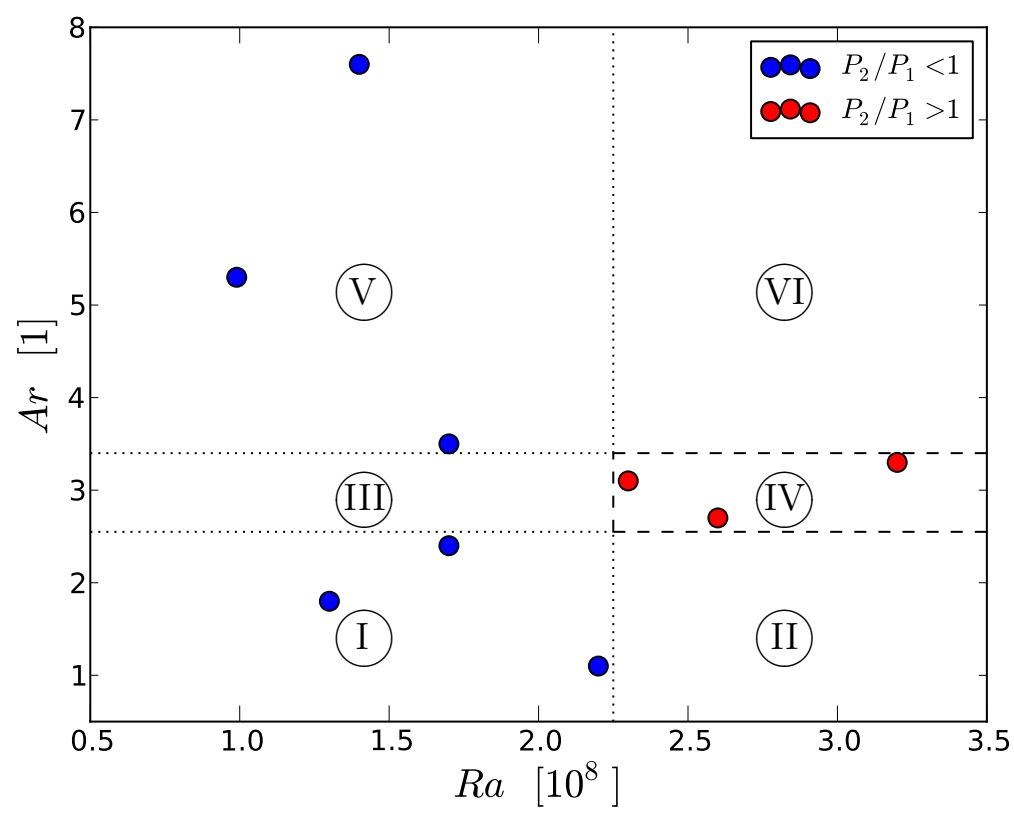

Figure 8.9: The plume fraction $P_{2} / P_{1}$ as a function of $R a$ and $A r$.

Figure 8.9 summarises the last two Figures 8.8 (e) and (f). Here, the plume fraction $P_{2} / P_{1}$ is colour coded and shown as a function of both $R a$ and $A r$. The dashed and dotted lines are guides to the eye and do not reveal sharp boundaries of the different regions. A plume fraction greater than one is obtained only in region IV, while in regions $\mathrm{I}$ and $\mathrm{V}$ the temperature abundance distribution is bulk dominated. However, Figure 8.9 reveals, that the compliance of either $R a \gtrsim 2.25 \times 10^{8}$, i.e. regions II, IV and VI, or $2.7 \lesssim A r \lesssim 3.3$, i.e. regions III and IV, might already be sufficient for a plume fraction greater than unity. For the parameter combinations in the regions II, III and VI we do not have any measurements. However, we measured $P_{2} / P_{1}>1$ only in region IV. Since two measurements reveal a bulk dominated distribution in the vicinity of region III, we expect this region to be bulk dominated as well. Accordingly, without the existence of further measurements $R a>2.2 \times 10^{8}$ seem to be an essential condition for the plumes to be dominant. It is still unclear what dominates the abundance distributions in regions II and VI. Hence, the open question whether a plume fraction greater unity occurs only in an intermediate $A r$ regime or for all $A r$ remains. Further measurements are necessary to address this question. 


\subsubsection{CONVECTIVE HEAT TRANSPORT}

Simultaneous measurements of temperature and velocity fields provide the distinguished possibility to determine instantaneous heat fluxes. Furthermore, the heat transport can be measured non intrusively, since both, combined PIT and PIV, are non intrusive techniques. The results presented here, however, must be interpreted with caution, since the field of view covers only a small fraction of the cross section of the sample and furthermore, the definition of the reference temperature is somehow arbitrary in MC. We choose the mean bulk temperature in the measurement layer $T_{b u l k}$ as reference temperature. This choice is reasonable, since it leads to a turbulent heat transport which is a result of bulk temperature fluctuations and temperature variations caused by passing plumes. According to the discussion in the previous section, the mean bulk temperature in the measurement layer is given by the position of the first Gaussian curve fitted to the temperature PDFs.

We calculate the normalised convective heat transport in the $x$-direction using $\dot{q}_{x} \cdot \rho^{-1}$. $c_{p}^{-1}=u \cdot\left(T-T_{b u l k}\right)$ and accordingly in the $y$-direction using the $v$-component of the velocity vector. Here, $\rho$ denotes the density and $c_{p}$ the specific heat capacity at constant pressure. The integrated values of the heat transport components are presented in Figure 8.10. The $x$-component is shown in (a) and (b), while (c) and (d) present the $y$-component. Obviously, no functional correlations between the $x$ and $y$-components of the normalised heat transports and $R a$ exist, see Figure 8.10 (a) and (c), which reveal bifurcations. Trends, however, complex ones, are identifiable as functions of $A r$, Figure 8.10 (b) and (d). The heat transport in $x$-direction, see Figure 8.10 (b), first increases towards zero with rising $A r$ before it jumps at $A r=2.7$ to high positive values. For higher $A r$ the heat transport has low positive values and decreases slowly. This change of the orientation of the heat flux at $A r \approx 2.7$, is most likely caused by a change of the LSC arrangement in the sample. The change from the FC dominated flow to a flow with a bypass comes along with a change of the LSC arrangement, which results in the different heat transport in the $x$-direction. For the $y$-component, Figure 8.10 (d), again, a change of the behaviour at $A r \approx 3$ is found.

In addition to the components of the heat transport, we present the integrated absolute values of it. These are calculated by $\sum_{x, y, t}|\dot{q}| \rho^{-1} c_{p}^{-1}=\sum_{x, y, t} \sqrt{\left(u^{2}+v^{2}\right) \cdot\left(T-T_{b u l k}\right)^{2}}$ and presented in Figure 8.11. They can be interpreted as the total amount of heat which is transported horizontally within the measurement layer. Here, a dependency of the absolute values of the heat transport is found on $R a$ as well, see Figure 8.11 (a). It increases up to $R a \approx 2.2 \times 10^{8}$ where a maximum is reached. Afterwards it stays almost constant. The strong change of the $R a$ scaling at $R a \approx 2.2 \times 10^{8}$ suits the change from bulk to plume dominated temperature fields, as discussed above. Accordingly, it seems that the total amount of heat transported horizontally within the measurement layer increases with $R a$ in bulk dominated regions, while it is almost constant for plume dominated ones. Figure 8.11 (b), reveals a local maximum of the integrated absolute values heat transport. It is located at $A r \approx 3$. This local maximum of the total amount of horizontally transported heat at $A r \approx 3$, suits as well the change of the LSC arrangement in the sample. Accordingly, both, 


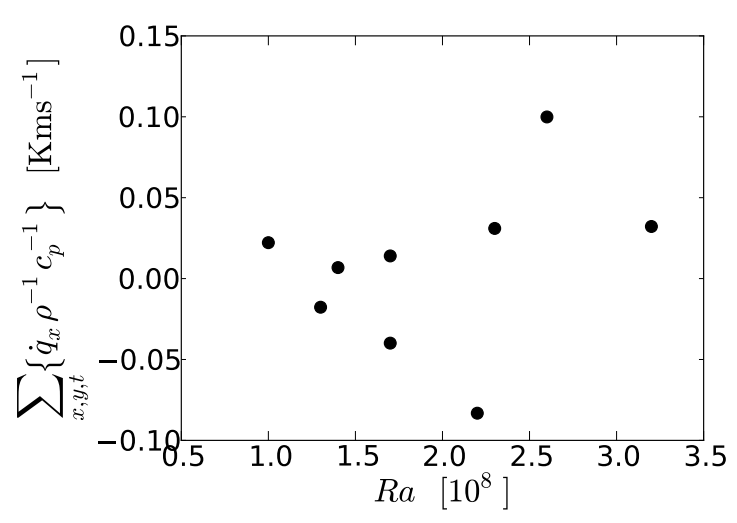

(a)

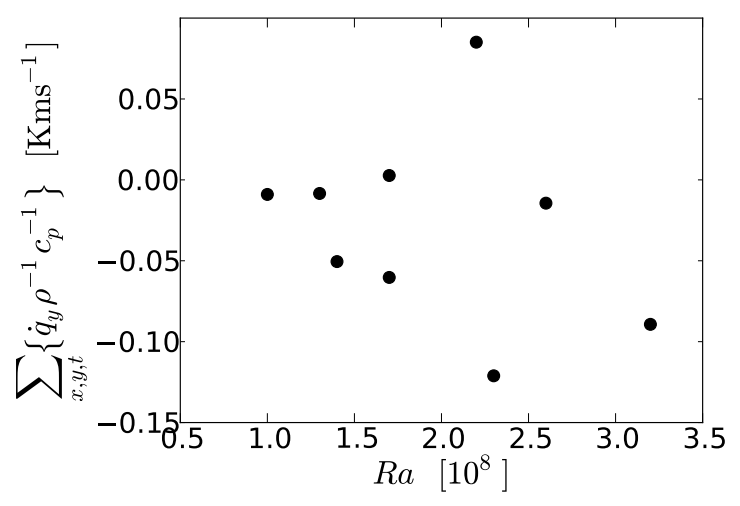

(c)

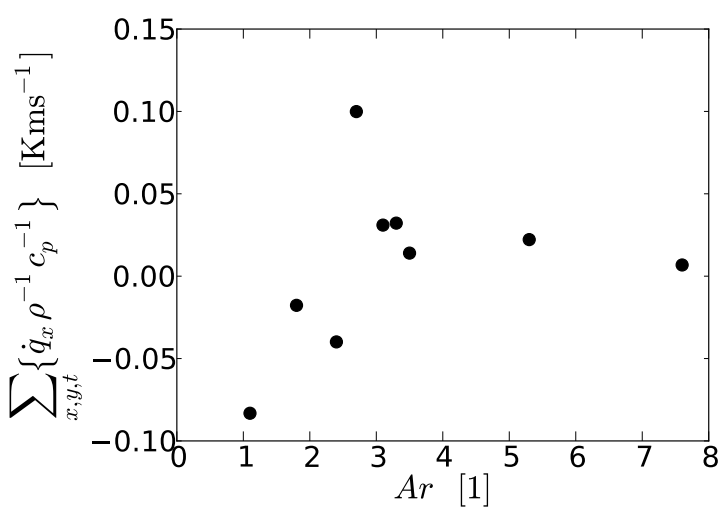

(b)

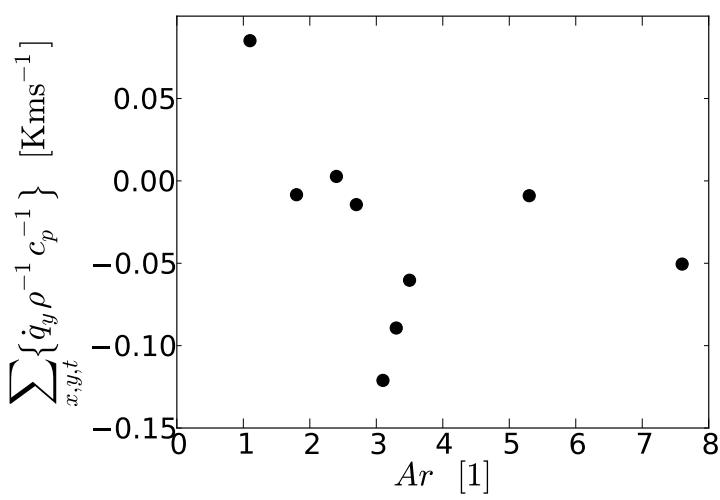

(d)

FIGURE 8.10: Integrated normalised convective heat transport components $\sum_{x, y, t} \dot{q}_{i} \rho^{-1} c_{p}^{-1}=$ $\sum_{x, y, t} \overrightarrow{u_{i}} \cdot\left(T-T_{b u l k}\right)$ within the measurement plane, with $i=u, v$, left as a function of $R a$ and right as a function of $A r$. (a) and (b) in $x$-direction as well as (c) and (d) in $y$-direction.

$R a \approx 2.3 \times 10^{8}$ and $A r \approx 2.7-3$ seem to be important numbers, where many quantities of the flow within the sample reveal strong changes.

Finally, it should be noted, that an increase of the vertical heat transport with rising $R a$ is expected for the plume dominated region as well. However, this could not be captured during these measurements since the vertical velocity component is not recorded.

\subsubsection{MEAN FIELDS}

In addition to the average velocity and temperature values, which are summarised in table 8.1. the mean velocity and temperature fields as well as the standard deviation of the temperature are depicted in Figure 8.12 for three parameter combinations. The first to be mentioned is the change in the overall flow direction. For case A05, shown in Figure 8.12 (a) the flow is directed towards the rear of the sample in the measurement plane, while it points in the opposite direction for case A09 in Figure 8.12 (c) and case A13 in Figure 8.12 (e). Furthermore, in Figure 8.12 (a), the region of descending air, entering the 


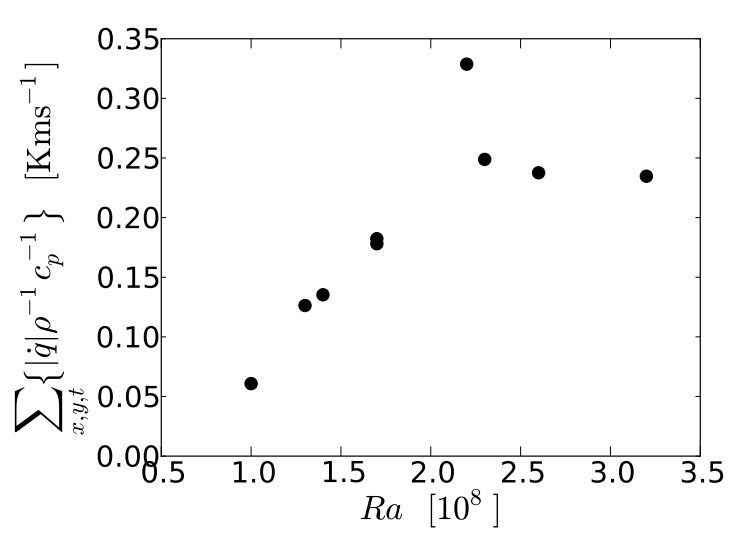

(a)

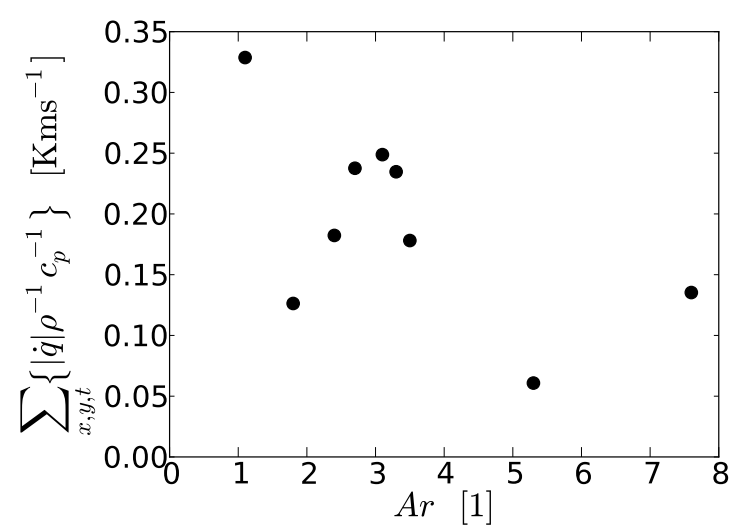

(b)

FiguRE 8.11: Integrated absolute values of the normalised convective heat transport $\sum_{x, y, t}|\dot{q}| \rho^{-1} c_{p}^{-1}=\sum_{x, y, t} \sqrt{\left(u^{2}+v^{2}\right) \cdot\left(T-T_{b u l k}\right)^{2}}$ within the measurement plane. (a) As a function of $R a$ and (b) as a function of $A r$.

measurement plane from above, is located at $y \approx 60 \mathrm{~mm}$ and strongest at $x>1000 \mathrm{~mm}$. From this point, and the region around $y \approx 60 \mathrm{~mm}$ the flow approaches mainly the rear of the cell. A small fraction forms a corner vortex for $y \lesssim 60 \mathrm{~mm}$. Different arrangements of the LSCs are known to occur in the convection sample, e.g. three or four rolls and an alignment in a "W-shape" 67, 98. However, to identify the arrangement based on the mean flow fields, yields large uncertainties since the field of view is small. Nevertheless, the observed flow field would suit an arrangement of four rolls in a "M" alignment, or even a five roll arrangement with cold air descending close to the front wall at $x \approx\{0,1000,2000\} \mathrm{mm}$ and warm air rising in the rear of the sample at $x \approx\{500,1500,2500\} \mathrm{mm}$.

The comparison of A09 and A13 reveals a similar mean velocity field and only slightly higher temperatures for A13. However, the standard deviation of the temperature is significantly higher for A09 in Figure 8.12 (d) than for A13 in Figure 8.12 (f). At similar $A r \approx 3.2$, A13 is characterised by both, higher $R a$ and higher Re. Accordingly, the thermal boundary layer thickness is expected to be smaller. Therefore, in relation to the boundary layer thickness the measurement plane is located further away from the thermal boundary layer within the bulk of the sample and thus smaller temperature fluctuations are observed.

Concluding this section, we calculated the angle $\alpha$ of the mean flow direction within the measurement plane. Thereby, $\tan (\alpha)=\langle v\rangle_{x, y} /\langle u\rangle_{x, y} \in\left(-180^{\circ}: 180^{\circ}\right]$ is defined as the angle between the $x$-axis and the velocity vector in the mathematical positive sense, i.e. $\alpha=0^{\circ}$ equals the positive $x$-direction, $\alpha=90^{\circ}$ equals the positive $y$-direction and $\alpha=-90^{\circ}$ equals the negative $y$-direction and so on, see inset in Figure 8.13 (b). The quadrants in the $u-v$-space are labelled in the standard way, i.e. quadrant I for $\langle u\rangle_{x, y, t}>0$ and $\langle v\rangle_{x, y, t}>0$. Quadrants II to IV follow in positive mathematical sense.

Figure 8.13 (a) presents the time averaged angles as a function of $R a$ revealing the existence of two "paths", which seem to collapse for $R a>2.6 \times 10^{8}$. However, it must be 


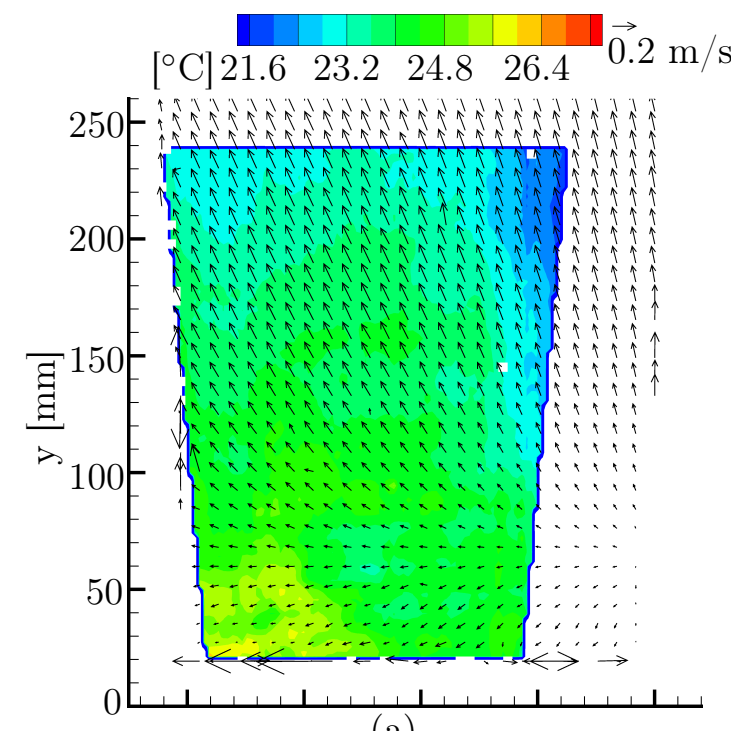

(a)
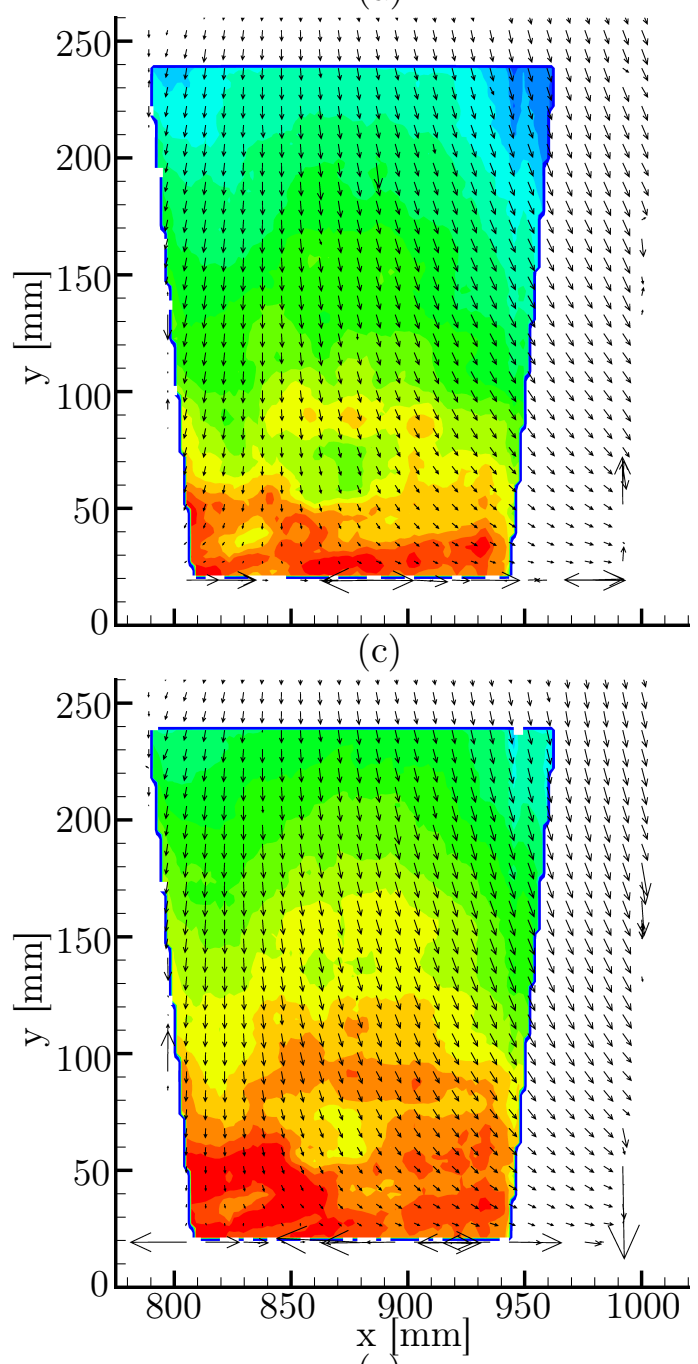

(e)

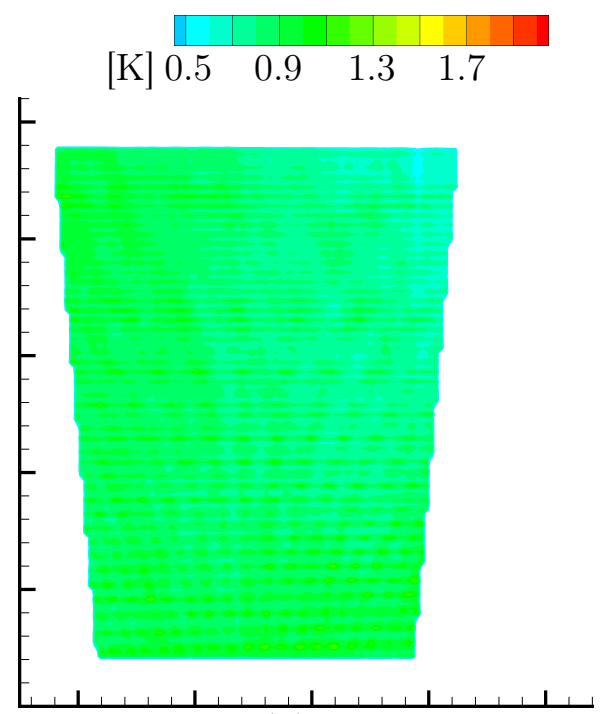

(b)

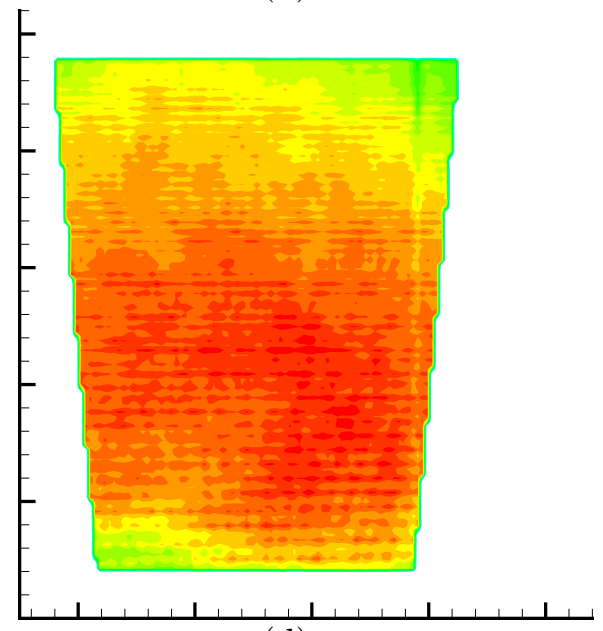

(d)

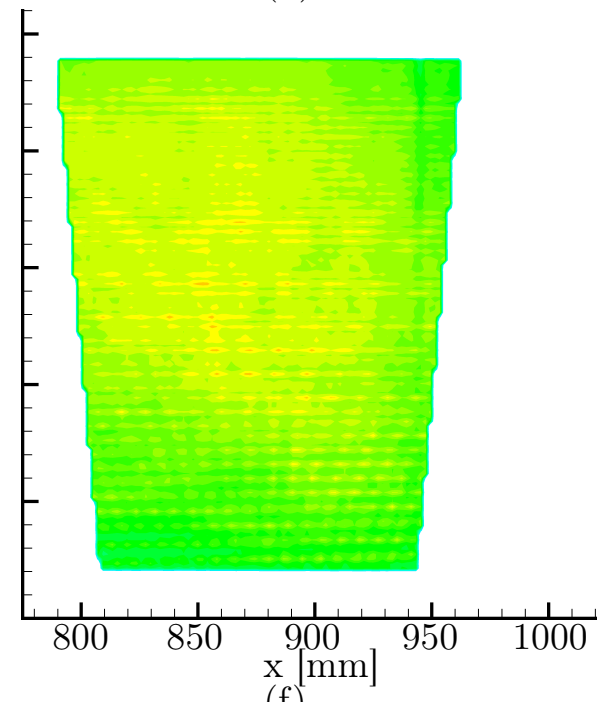

(f)

Figure 8.12: Mean fields (left) and standard deviation of the hue (right). (a) and (b): A05, (c) and (d): A09 and (e) and (f): A13. 


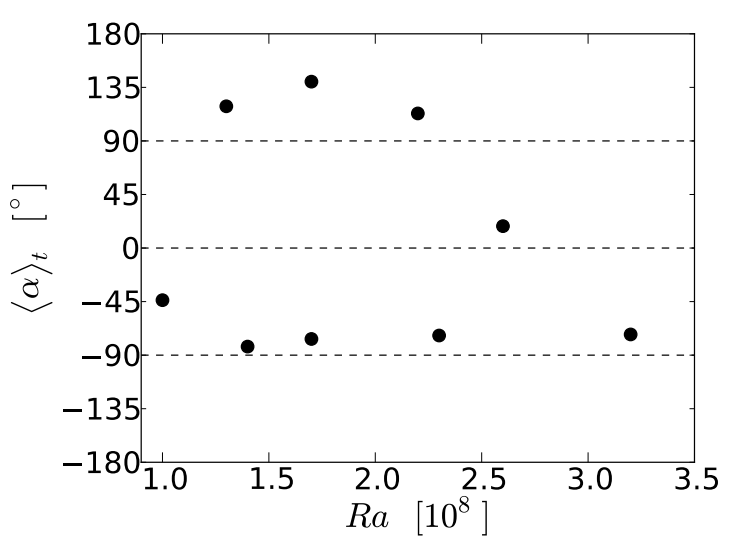

(a)

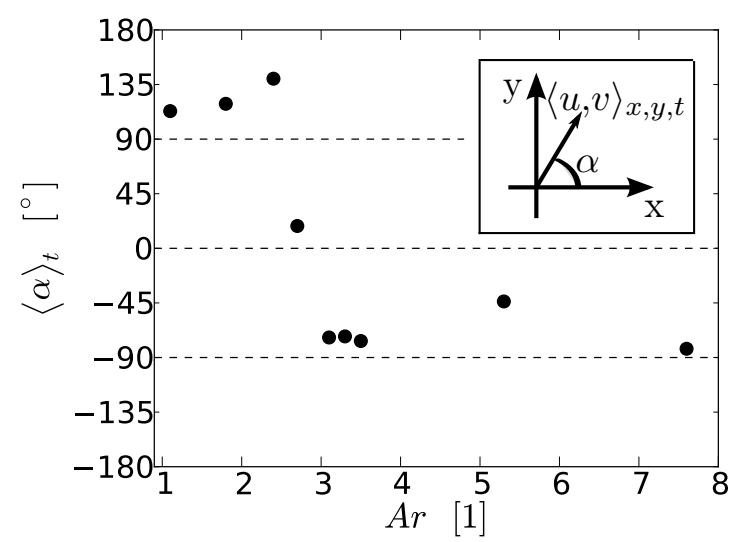

(b)

FIgURE 8.13: Time averaged angle $\alpha$ of the mean flow direction (a) as a function of $R a$ and (b) as a function of $\mathrm{Ar}$.

noted, that only one measurement was conducted for $R a>3 \times 10^{8}$, thus, it is possible, that more measurements would reveal angles of approximately $120^{\circ}$ at high $R a$ as well. Accordingly, the paths would not collapse for high $R a$. The time averaged angles plotted over $A r$ in the Figure 8.13 (b) reveal a clear dependency. For low $A r<2.5$ we find an angle in the II quadrant of approximately $120^{\circ}$. In contrast, we observe for high $\mathrm{Ar}>3$ angles in the IV quadrant of approximately $-75^{\circ}$. Only for the intermediate $A r=2.7$ we observe a value of $18^{\circ}$ in the I quadrant which strongly deviates from all other values. The found conversion is headed from negative to positive angles, more specific from the II to the IV quadrant. It can be explained by the occurrence of the "bypass" flow for high $A r$. Surprisingly, the angle changes by approximately $180^{\circ}$, indicating regions with a given $x$ position close to front wall, where cold air descents for low $A r$, turn to regions where warm air rises for high $A r$. The same holds for the regions in the rear of the sample. No strong change of the $x$ position takes place.

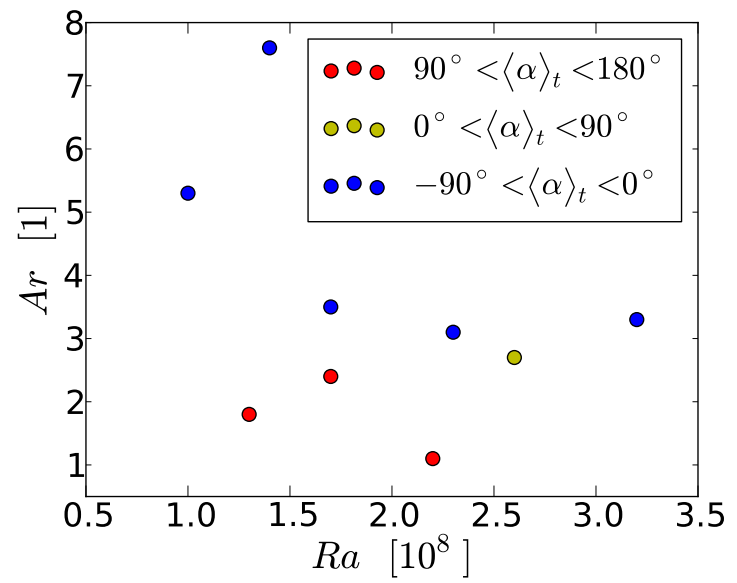

Figure 8.14: Time averaged angle $\alpha$ of the mean flow direction as a function of $R a$ and $A r$. 
Summarising, in Figure 8.14 the colour coded angles are plotted as a function of both $R a$ and $A r$. Here, the dependency of $\alpha$ on $A r$ only is obvious. The change from a "regular" forced convective roll to a "bypass" flow occurs at $A r \approx 2.7$, as shown in Figures 8.13 (b) and 8.14 . This is the same value, where the plume fraction $P_{2} / P_{1}$ changes significantly, Figure 8.8 (f). Accordingly, the conversion of the mean flow direction, correlates which a change of the temperature abundances from bulk to plume dominated distributions. We expect the plume fraction, i.e. the number density of the plumes close above the bottom thermal boundary layer, to be the trigger for the conversion of the mean flow. However, it might also be vice versa. Further measurements could help to confirm or disprove our interpretation.

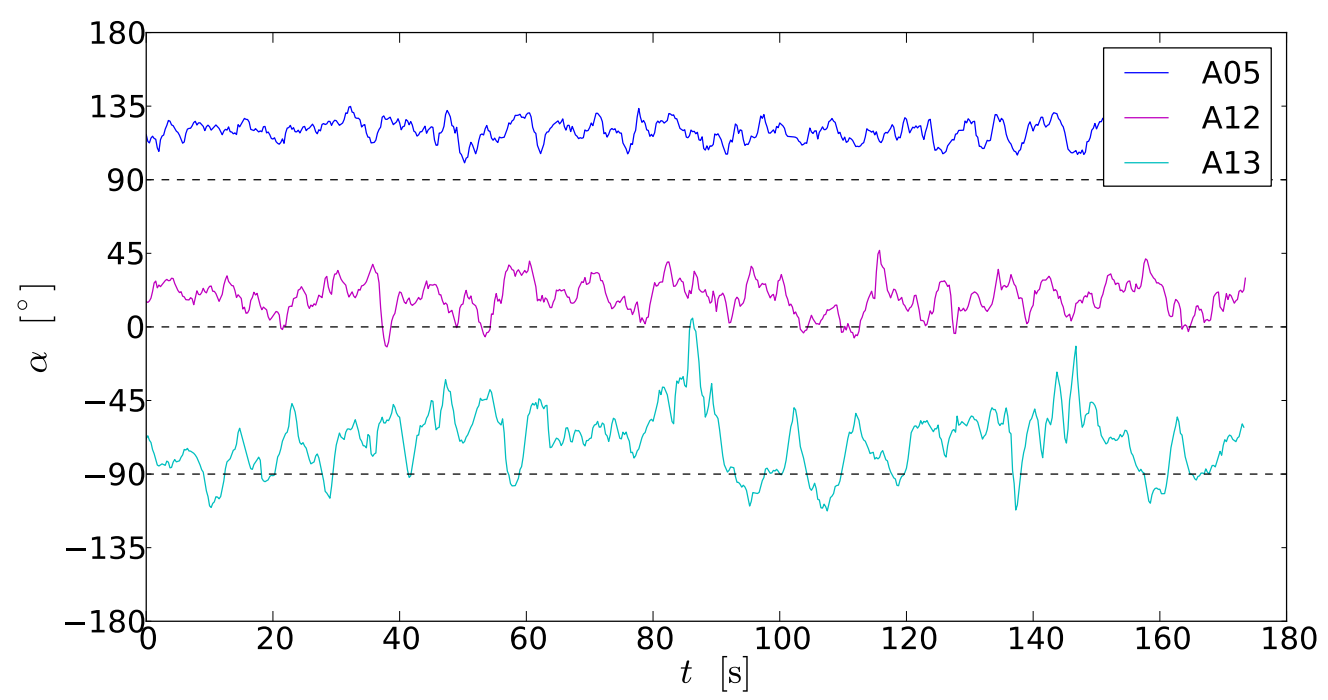

Figure 8.15: Time series of the angle $\alpha$ of the mean flow direction. Instead of A11 and A09 which are qualitatively very similar to A05 (-) and A13 (-), respectively, we added A12 (-), which shows a different behaviour.

The time evolution of $\alpha$ based on the spatially averaged velocity fields is presented in Figure 8.15. We depicted one representative case for each quadrant. Hence, we omit the cases A11 and A09, which are qualitatively and quantitatively very similar to A05 and A13, respectively. Therefore, we added A12, which was the only case with an angle between zero and $90^{\circ}$. The presented cases are all recorded at $R e \approx 1.1 \times 10^{4}$ and increasing $\mathrm{Ar}$ (and $R a$ ) from A05 to A12 to A13. The dashed lines mark the borders of the quadrants in the $u-v$ space. An intersection of the instantaneous angle with such a dashed line indicates a change of the main flow direction. These do not occur for A05 for which the fluctuations of the angle are additionally low. For A12, the mean angle is approximately $18^{\circ}$, i.e. a flow direction in the I quadrant. However, for few short time periods, the mean flow direction changes in IV quadrant. For the case A13, the strongest fluctuations of the angle occur accompanied by a change from the IV to the I and many to the III quadrant. Thus, we showed that besides the $A r$ dependency of time averaged angle also $\alpha(t)$ has a strong dependency on $A r$. This includes more changes of the main flow direction with 
increasing $A r$ and higher fluctuations of $\alpha$ in general.

An identification of the arrangement of the LSCs in the convection sample, e.g. three or four rolls and an alignment in a "W-shape" [67, 98], based on the detected angle yields too large uncertainties since the field of view is so small. However, the existence of a mean angle different from a multiple of $90^{\circ}$ substantiates the arrangement of the LSCs in a "W-shape" or something similar.

\subsection{SUMMARY AND CONCLUSION}

We applied PIT-PIV to simultaneously measure the velocity and temperature fields of mixed convection in a cuboidal sample with width, length and height amounting to 500 $\mathrm{mm}, 2500 \mathrm{~mm}$ and $500 \mathrm{~mm}$, respectively. The parameter range covers $R e=0.5-1.7 \times 10^{4}$, $R a=1.0-3.2 \times 10^{8}$ and $A r=1.1-7.6$ at $\operatorname{Pr} \approx 0.7$. The measured field of view is located close above the bottom thermal boundary layer and has lateral dimensions of approximately $200 \mathrm{~mm} \times 150 \mathrm{~mm}$. A time resolution of $0.25 \mathrm{~s}$ is realised.

The thermal fingerprints of sheet-like plumes and stems of mushroom like plumes are visualised based on instantaneous temperature and velocity fields. Herein, we found that the sheet-like plumes align with the shear for rather low $R a$. At higher $R a$, this correlation vanishes, however, the fingerprints of mushroom like plumes are still visible in the temperature and velocity fields.

For a more statistic analysis of the plumes, temperature PDFs are evaluated. Two Gaussian curves are used to fit the distribution functions. The plume fraction $P_{2} / P_{1}$, i.e. the ratio of the abundances of the temperatures related to the plumes compared to those of the bulk, reveals a steep change at $R a_{c} \approx 2.25 \times 10^{8}$. It corresponds to an intermediate Ar regime, $2.7 \lesssim A r \lesssim 3.3$. The balance of buoyancy and inertia forces in combination with a turbulent bulk flow seems to be the essential ingredient for this change. A further role plays the height change of the measurement plane compared to the thickness of the bottom thermal boundary layer with increasing $R a$. Together with the increasing bulk fluctuations a stronger plume fragmentation process, as proposed by Bosbach et al. [18], causes the change of the temperature distributions. The found intermediate $A r$ regime for instabilities of the flow in this convection sample confirms similar finding by Schmeling et al. 98.

Again at $A r \approx 2.7-3$, we observe a change in the horizontal heat fluxes. These could be determined since temperature and velocity fields were recorded simultaneously. Furthermore, the total amount of heat transported horizontally within the measurement layer increases with $R a$ in bulk dominated regions, while it stays almost constant for plume dominated ones.

Finally, an evaluation of the mean flow angle $\alpha$ within the measurement plane, reveals a dependency on $A r$ only, with a steep change at $A r=2.7$. For lower $A r$ the dominating forced convection imprints positive averaged $v$-components, while higher $A r$ result in negative ones, i.e. a bypass flow. This is also in agreement with the results of Schmeling et al. [98. Furthermore it is shown that for increasing $A r$ the fluctuations of $\alpha$ increase as 
well. In specific, this includes more frequent changes of the main flow direction between the different $u-v$ quadrants. 


\section{PART III}

\section{CONCLUSION}

9 SUMMARY AND CONCLUSION

10 OUTLOOK 



\section{SUMmARY AND CONCLUSION}

In the accumulated papers of this thesis, new insights on mixed convective air flows are achieved. Furthermore, a novel measurement technique for the investigation of these systems is presented. To summarise the accomplished tasks, the six questions formulated in the motivation section will be addressed with respect to the results of the single chapters.

"How do LSCs arrange in a cuboidal convection sample of aspect ratio five as a function of the characteristic numbers?"

Based on PIV (Chapter 4), point-wise temperature measurements (Chapters 5 and 6 ) and combined PIT/PIV (Chapter 8) the existence of a strong dependence of the mean flow field on the ratio of inertia to buoyancy forces, i.e. Ar, was found. For FC dominated flows, the air flow in the cell is governed by a nearly two-dimensional solid body like rotation with separation regions in the cell corners. Upon the onset of TC, a collapse of the two-dimensional mean wind was observed, as well as a formation of four convection rolls, which interacted with the wall jet of the inflowing air. A POD of the instantaneous velocity fields confirmed the existence of these large-scale coherent structures. Moreover, the POD results show that the onset of a buoyancy induced secondary flow leads to a decrease of the first mode energy as well as an increased number of modes containing more than $1 \%$ of the total energy. The observed flow structures were also visible in the temperature series measured in the air outlet.

Furthermore, an arrangement of the four convection rolls in a ' $\mathrm{W}$ ' or ' $\mathrm{M}$ ' configuration was found in Chapter 6. This is in agreement with tomographic PIV measurements conducted by Kühn et al. [67]. Recent investigations using combined PIT/PIV (Chapter 8) in a rather small field of view support these findings. In addition to these four convection rolls, parameter combinations were found, which result in a three convection roll configuration in the sample.

Finally, in Chapter 8 , an evaluation of the angle $\alpha$ of the mean flow within the measurement plane, revealed a dependency on the characteristic number $A r$ only. A strong change at $A r=2.7$ was found. For lower $A r$, the dominating FC imprinted a positive time and space averaged $v$-component, while higher $A r$ resulted in negative ones, i.e. a bypass flow. This finding is in agreement with the results in Chapter 6. Furthermore, it was found that increasing $A r$ causes stronger fluctuations of the angle $\alpha$. Specifically, this includes more changes of the main flow direction between the different quadrants in the $u-v$ space, i.e. reversals of at least one component of the main flow direction. 
"How does the enthalpy transport between in- and outflowing air depend on the Archimedes number?"

The analysis of the temperature difference between in- and outflowing air (Chapter 4) revealed the existence of an upper boundary. First it increased with rising $A r$ and then approached a constant value for $A r>0.9$. Different $A r$ were realised by adjusting $R e$ at a constant $R a$. Hence, this saturation indicates that the resulting temperature difference between in- and outflowing air is governed by buoyancy forces in this range of $A r$, while inertia forces, if at all, play only a minor role. As a consequence a change of $A r$, and thus $R e$, will not alter the temperature difference in this range. This finding is in good agreement with the observed plateau-like behaviour of rms-values of the $w$-component slightly above the bottom plate at $Z=0.1 \times H$ for $A r<1$ and its steep increase for $A r>1$. These rms-values are a measure of fluctuations of the vertical velocity component and result from increasing buoyancy forces as compared to inertia forces. Finally, as a result of the upper boundary for the temperature difference between out- and inflowing air and the decreasing $R e$ for increasing $A r$, a maximum in the enthalpy flux of the fluid was found at $A r \approx 0.6$.

"How can the stability as well as the dynamics of the LSCs be characterised and in which ways do the dynamics scale with the characteristic numbers?"

Oscillations of the temperature signals at frequencies below $2.0 \times 10^{-3} \mathrm{~s}^{-1}$ were found during long-term temperature measurements (Chapters 5 and 6). These temperature fluctuations are ascribed to oscillations and erratic reorientations of the LSCs in the flow. Stable temperature configurations, periodic oscillations as well as spontaneous reversals and reorientations were detected. In the case of periodic oscillations, interpretation of the temperature signals in combination with smoke visualisations determined that the LSC arrangement changes between three and four thermally induced convection rolls.

A simple statement on whether oscillations will occur can be summarised as follows: The smaller $R e$ and the smaller $R a$ the higher is the possibility for the occurrence of oscillations. Furthermore, with increasing $R a$, oscillations occur at higher $R e$ and have higher frequencies.

In more detail, it turned out that the effective Archimedes number based on the temperature difference between heated bottom and inflowing air seemed to be a better parameter to predict the occurrence of periodic oscillations. These are only detected in an intermediate regime of $A r_{\text {eff }}$; accordingly equilibrium between inertia and buoyancy forces is an essential ingredient for the development of these oscillations. Hence, the oscillations are triggered by the interaction of the forced convective wall jet at the ceiling with the thermally driven LSCs. However, a comparable strength of buoyancy and inertia forces is not an adequate criterion, since further $R a$ must not be too high. Otherwise only steady configurations are found. Frequency analysis of the sensor signals reveals a pronounced main frequency and smaller peaks at multiples of it. Further, the frequency scales linearly with $R e$, which is based on the inflow velocity, for those cases where periodic oscillations occur. This reflects that the FC contribution determines the frequency of the oscillations. 
The maximal observed main frequency of the oscillations reached a value of $f=2.0 \times$ $10^{-3} \mathrm{~s}^{-1}$ at $A r=1.8\left(A r_{\mathrm{eff}}=2.1\right)$ and $R a=2.0 \times 10^{8}\left(R e=1.3 \times 10^{4}\right)$. This oscillation frequency is about one order of magnitude smaller compared to the turnover frequencies of both wall jet and LSCs.

An important result regarding the dynamics of the LSCs is that the oscillations of the LSCs developed due to the formation of a new LSC next to one side wall followed by a break-down of a LSC next to the other side wall. Consequently, the LSCs coupled the momentum transport at opposing side walls. Further, back-coupling linked LSCs breaking down at one side wall to formation of new LSCs at the other side wall. Even more, stable LSCs as well as periodically oscillating LSCs could be destroyed by adjusting the inflow velocity, that is, $R e$.

A concept to describe the mechanisms acting to trigger the periodic oscillations is presented in Chapter 6, which illustrates the convection rolls as elastic rubber straps known from classical mechanics.

"How can temperature and velocity fields be simultaneously recorded in a MC airflow?"

The usage of TLCs as tracer particles for combined PIT and PIV is presented as a novel tool for the investigation of $\mathrm{MC}$ air flows in Chapter 7. The technique allows simultaneous recording of planar instantaneous temperature and velocity fields. In order to accomplish the measurements, different methods of particle generation were studied and suitable white light sheet optics were developed. The latter generate a white light sheet with a thickness (fwhm) of approximately $9 \mathrm{~mm}$, very low divergence and a rather homogeneous illumination over a distance of $500 \mathrm{~mm}$. It is based on LEDs, which guarantee a fast response within less than $50 \mu$ s to the trigger signal at rather low cost.

In order to separate the temperature information from the background and pixel noise, a filtering procedure for the recorded colour data was developed. It is based on averaging the hue over interrogation windows while taking into account only those pixels with a $V$ value (HSV) above a certain local threshold. Its working process, applicability and benefits were demonstrated on a synthetic image.

In order to perform quantitative measurements, a fast calibration procedure was developed and employed, which provides a calibration accuracy of the absolute temperatures of $\sigma_{T}=0.19 \mathrm{~K}$ in the studied temperature range of $20.5-21.7^{\circ} \mathrm{C}$. By considering the errors of the calibration curve and the hue noise, which is computed from the measurement data, a standard error for relative temperature measurements as low as $\sigma_{\Delta T}=0.06 \mathrm{~K}$ is calculated, corresponding to a temperature dynamic range of 20 . The dynamic range on the hue scale, however is much higher and amounts to 43, revealing an even higher potential of the technique, depending on the accuracy of the hue temperature calibration.

The application of this technique in a horizontal measurement plane in order to study the dynamics of thermal plumes is discussed in Chapter 8. For this, R20C20W (Hallcrest) TLCs were used, which reveal temperature sensitive properties in a larger temperature range of approximately $21-29^{\circ} \mathrm{C}$. However, a loss in absolute and relative accuracy had 
to be taken into account.

"What is the time evolution of, for example, a warm rising plume in this enclosure?"

Results from combined PIT/PIV of convective air flows are presented and discussed in Chapter 7. Amongst others, a thermal plume (including its mushroom structure, the adjacent vortices, and its root with quickly rising warm air) was captured with high spatial resolution at $R a \approx 9.0 \times 10^{7}$. Furthermore, due to a time resolution of $4 \mathrm{~Hz}$, a large fraction of its dynamics were resolved. This plume was measured in the absence of an externally applied pressure gradient, i.e. $R e=0$. The breakdown of the typical plume structure towards a thin region of warm rising air was captured.

"How do the Rayleigh and the Archimedes numbers affect the dynamics of sheet-like plumes?"

The thermal fingerprints of sheet-like plumes and of the stems of mushroom-like plumes were visualised in instantaneous temperature and velocity fields. Herein, the alignment of the sheet-like plumes with the shear for rather low $R a$ was shown. At higher $R a$, this correlation vanished, however, the fingerprints of the stems of mushroom-like plume could be detected in the temperature and velocity fields.

An analysis of the statistics of the plumes in terms of PDFs of the temperature is presented in Chapter 8. A sum of two Gaussian curves is used to fit the distribution functions. A strong change of $P_{2} / P_{1}$, i.e. the ratio of the areas under the Gaussian curves, is found at $R a_{c} \approx 2.3 \times 10^{8}$. It corresponds to an intermediate $A r$ regime, $2.7 \lesssim A r \lesssim 3.3$. The balance of buoyancy and inertia forces in combination with sufficiently turbulent bulk flow seems to be the essential ingredient for this change. A further role is played by the change of the height of the measurement plane compared to the thickness of the bottom thermal boundary layer with increasing $R a$. Together with the increasing bulk fluctuations a stronger plume fragmentation process, as proposed by [18], causes the change of the temperature distributions. 


\section{OUTLOOK}

This chapter is divided into two parts. The first addresses the issue of MC in the cuboidal convection sample with respect to further analysis and investigation of open questions. The second part discusses further progress of the combined PIT/PIV technique.

Concerning structure formation and dynamics of the LSCs in MC in a cuboidal convection sample, the aspect ratio dependence is still an open issue. Hence, reducing the aspect ratio $\Gamma_{x z}$ of the investigated sample from five to lower values should be addressed in a future study. The questions of how many LSCs arranged in MC as a function of $\Gamma_{x z}$ and how they are oriented can be tackled. Furthermore, long-term measurements will provide information on the LSC dynamics and its dependence on $\Gamma_{x z}$.

The Chapters 4 and 6 suggest a more detailed analysis of interaction between wall jet and buoyancy induced LSCs. Long-term measurements using temperature probes and/or laser Doppler anemometry could help in drawing conclusions concerning this open question.

In Chapter 8 , the occurrence of a critical $R a$ is explained with different relative heights of the measurement plane compared to the boundary layer thickness. Accordingly, measurements of the plume dynamics for constant parameter combinations as a function of the height of the horizontal measurement plane should be conducted. These will clarify the dependence of the plume statistics on the distance between thermal boundary layer and position of the measurement plane.

In order to address the question posed in Chapter 8 , i.e. whether a plume fraction greater than unity occurs for $R a>2.3 \times 10^{8}$ only in an intermediate $A r$ regime or for all $A r$, the extension of the parameter range of the current study to regions II and VI (see Chapter 8) is desirable. Furthermore, measurements in region III could confirm the expected bulk dominated distribution therein.

With regard to combined PIT/PIV (see Chapter 7) the next steps in maturing the technique include incorporating further research on the hue-temperature calibration in order to push the limits in terms of increased precision as well as the optimisation of the measurement and evaluation parameters for higher dynamic ranges of the hue values. Another issue, which has to be addressed to establish the technique, is a further characterisation of TLC particles in air flows. The colour play in particular, in which the dispersed TLC particles deviate from the bulk properties, should be studied in more detail. Knowledge and control of the size dependence of the colour play could help to optimise the measurement and dynamic range in the future. Also, measurement of the exact reaction times of the particles is required to assess the potential of the technique for application in terms of higher velocities. 
As noted in Chapter 8, a larger field of view is desirable, since less extrapolation and therefore more precise interpretation of the results will be possible by recording a larger fraction of the cross sections of the sample. Furthermore, a next step should be the simultaneous measurement of the vertical velocity component with the temperature field in horizontal measurement planes, i.e. the combination of stereoscopic PIV (3C-2D) with PIT. This would allow a detailed survey of the heat transport and the dynamics of thermal plumes slightly above the boundary layer.

The presented novel measurement technique, including the open tasks for its progression, provide a variety of new prospects for the investigation of convective flows. In particular, a better understanding of the physical processes in turbulent temperature driven air flows can aid in making an important contribution to technical applications like the optimisation of air conditioning for rooms, vehicles or entire buildings regarding comfort and environmental protection. 


\section{BIBLIOGRAPHY}

[1] C. Abram, B. Fond, A.L. Heyes, and F. Beyrau. High-speed planar thermometry and velocimetry using thermographic phosphor particles. Appl. Phys. B, 111:155-160, 2013.

[2] G. Ahlers, E. Bodenschatz, D. Funfschilling, and J. Hogg. Turbulent Rayleigh-Bénard convection for a Prandtl number of 0.67. J. Fluid Mech., 641:157-167, 2009.

[3] G. Ahlers, S. Grossmann, and D. Lohse. Heat transfer and large scale dynamics in turbulent Rayleigh-Bénard convection. Rev. Mod. Phys., 81:503-537, 2009.

[4] H.D. Baehr and K. Stephan. Wärme und Stoffübertragung. Springer-Verlag, 3rd edition, 1998.

[5] J. Bailon-Cuba, O. Shishkina, C. Wagner, and J. Schumacher. Low-dimensional model of turbulent mixed convection in a complex domain. Phys. Fluids, 24:107101, 2012.

[6] M. Barth and A. Raabe. Acoustic tomographic imaging of temperature and flow fields in air. Meas. Sci. Technol., 22:035102, 2011.

[7] S. Baskaya, U. Erturhan, and M. Sivrioglu. Experimental investigation of mixed convection from an array of discrete heat sources at the bottom of a horizontal channel. Int. J. Heat Mass Transfer, 42:56-63, 2005.

[8] G.K. Batchelor. The theory of homogenious turbulence. Cambridge University Press, 1st edition, 1959.

[9] T.P. Bednarz, C. Lei, and J.C. Patterson. An experimental study of unsteady natural convection in a reservoir model subject to periodic thermal forcing using combined PIV and PIT techniques. Exp. Fluids, 47:107-117, 2009.

[10] H. Bénard. Les tourbillons cellulaires dans une nappe liquide. Rev. Gén. Sciences Pure Appl., 11:1261-1271 and 1309-1328, 1900.

[11] D. Bercovici. The generation of plate tectonics from mantle convection. Earth Planet. Sci. Lett., 205:107-121, 2003.

[12] P. Bonnet. Applications of liquid crystals in aerodynamic testing. Phd thesis, University of Oxford, Oxford, United Kingdom, 1989.

[13] J. Bosbach, A. Heider, T. Dehne, M. Markwart, I. Gores, and P. Bendfeldt. Evaluation of cabin displacement ventilation under flight conditions. In 28th Int. Congress of the Aeronautical Sciences, Brisbane, Australia, 2012.

[14] J. Bosbach, M. Kühn, and C. Wagner. Large scale particle image velocimetry with helium filled soap bubbles. Exp. Fluids, 46:539-547, 2009.

[15] J. Bosbach, S. Lange, T. Dehne, G. Lauenroth, F. Hesselbach, and M. Allzeit. Alternative ventilation concepts for aircraft cabins. In Deutscher Luft- und Raumfahrtkongress, Berlin, Germany, 2012. 
[16] J. Bosbach, S. Lange, T. Dehne, G. Lauenroth, F. Hesselbach, and M. Allzeit. Alternative ventilation concepts for aircraft cabins. CEAS Aeronaut. J., 2013.

[17] J. Bosbach, J. Pennecot, C. Wagner, M. Raffel, T. Lerche, and S. Repp. Experimental and numerical simulation of turbulent ventilation in aircraft cabins. Energy, 31:694-705, 2006.

[18] J. Bosbach, S. Weiss, and G. Ahlers. Plume fragmentation by bulk interactions in turbulent Rayleigh-Bénard convection. Phys. Rev. Lett., 108:054501, 2012.

[19] J.V. Boussinesq. Théorie analytique de la chaleur. Gauthier-Villars Paris, 1903.

[20] E. Brown and G. Ahlers. Rotations and cessations of the large-scale circulation in turbulent Rayleigh-Bénard convection. J. Fluid Mech., 568:351-386, 2006.

[21] E. Brown and G. Ahlers. Large-scale circulation model for turbulent Rayleigh-Bénard convection. Phys. Rev. Lett., 98:134501, 2007.

[22] E. Brown and G. Ahlers. Azimithal asymmetries of the large-scale circulation in turbulent Rayleigh-Bénard convection. Phys. Fluids, 20:105105, 2008.

[23] E. Brown and G. Ahlers. A model of diffusion in a potential well for the dynamics of the large-scale circulation in turbulent Rayleigh-Bénard convection. Phys. Fluids, 20:075101, 2008.

[24] E. Brown and G. Ahlers. The origin of oscillations of the large-scale circulation of turbulent Rayleigh-Bénard convection. J. Fluid Mech., 638:383-400, 2009.

[25] E. Brown, A. Nikolaenko, and G. Ahlers. Reorientation of the large-scale circulation in turbulent Rayleigh-Bénard convection. Phys. Rev. Lett., 95:084503, 2005.

[26] E. Buckingham. On physically similar systems; illustrations of the use of dimensional equations. Phys. Rev., 4:345-376, 1914.

[27] E. Buckingham. The principle of similitude. Nature, 96:396-397, 1915.

[28] S. Chandrasekhar. Hydrodynamic and Hydromagnetic Stability. Dover Publications, 1981.

[29] F. Chillà, M. Rastello, S. Chaumat, and B. Castaing. Long relaxation times and tilt sensitivity in Rayleigh-Bénard turbulence. Eur. Phys. J. B, 40:223-227, 2004.

[30] F. Chillà and J. Schumacher. New perspectives in turbulent Rayleigh-Bénard convection. Eur. Phys. J. E, 35:58, 2009.

[31] M. Czapp. Simultane Messung instantaner Geschwindigkeits- und Temperaturfelder von Rayleigh-Bénard-Konvektion. Diploma thesis, Georg-August-University, Göttingen, Germany, 2007.

[32] M. Czapp, J. Bosbach, A. Dillmann, C. Wagner, and H. Richard. Bestimmung von Temperaturen in einer Gasströmung. Patent-Nr. 102007035 499, 2007.

[33] D. Dabiri. Digital particle image thermography / velocimetry: a review. Exp. Fluids, 46:191-241, 2009.

[34] G.F. Davies. Whole-mantle convection and plate tectonics. Geophys. J. R. astr. Soc., 49:459-486, 1977. 
[35] J.R. de Bruyn, E. Bodenschatz, S.W. Morris, S. Trainoff, Y. Hu, D.S. Cannell, and G. Ahlers. Apparatus for the study of Rayleigh-Bénard convection in gases under pressure. Rev. Sci. Instrum., 67:2043, 1996.

[36] Zentrales Archiv des DLR.

[37] M.S. Emran and J. Schuhmacher. Conditional statistics of thermal dissipation rate in turbulent Rayleigh-Bénard convection. Eur. Phys. J. E, 35:108, 2012.

[38] B. Fond, C. Abram, A.L. Heyes, A.M. Kempf, and F. Beyrau. Simultaneous temperature, mixture fraction and velocity imaging in turbulent flows using thermographic phosphor tracer particles. Opt. Express, 20:22118-22133, 2012.

[39] F. Fontenele Araujo, S. Grossmann, and D. Lohse. Wind reversals in turbulent RayleighBénard convection. Phys. Rev. Lett., 95:084502, 2005.

[40] N. Fujisawa, S. Funatani, and N. Katoh. Scanning liquid-crystal thermometry and stereo velocimetry for simultaneous three-dimensional measurement of temperature and velocity field in a turbulent Rayleigh-Bénard convection. Exp. Fluids, 38:291-303, 2005.

[41] N. Fujisawa and Y. Hashizume. An uncertainty analysis of temperature and velocity measured by a liquid crystal visualization technique. Meas. Sci. Technol., 12:1235-1242, 2001.

[42] N. Fujisawa, Y. Watanabe, and Y. Hashizume. Visualization of turbulence structure in unsteady non-penetrative thermal convection using liquid crystal thermometry and stereo velocimetry. J. Visualization, 11(2):173-180, 2008.

[43] A.V. Getling. Rayleigh-Bénard Convection, volume 11 of Advanced series in nonlinear dynamics. (C)1998, World Scientific Publishing.

[44] P. Gil, F. Torres, F.G. Ortiz, and O. Reinoso. Detection of partial occlusions of assembled components to simplify the disassembly tasks. Int. J. Adv. Manuf. Technol., 30:530-539, 2006 .

[45] K. Goelker. GIMP 2.6 for photographers: image editing with open source software. Rocky Nook, 1 st edition, 2011.

[46] D.D. Gray and A. Giorgini. The validity of the boussinesq approximation for liquids and gases. Int. J. Heat Mass Transfer, 19:545-551, 1976.

[47] G.S. Grewal, M. Bharara, J.E. Cobb, V.N. Dubey, and D.J. Claremont. A novel approach to thermochromic liquid crystal calibration using neural networks. Meas. Sci. Technol., 17:1918-1924, 2006.

[48] S. Grossmann and D. Lohse. Scaling in thermal convection: a unifying theory. J. Fluid Mech., 407:27-56, 2000.

[49] G. Hadley. Concerning the cause of the general trade winds. Phil. Trans. Roy. Soc. London, 29:58-62, 1735.

[50] Hallcrest. Handbook of thermochromic liquid crystal technology, 1991.

[51] T. Haramina and A. Tilgner. Coherent structures in boundary layers of Rayleigh-Bénard convection. Phys. Rev. E, 69:056306, 2004.

[52] D.P. Hart. PIV error correction. Exp. Fluids, 29:13-22, 2000. 
[53] W.J. Hiller, S. Koch, and T.A. Kowalewski. Simultane Erfassung von Temperatur- und Geschwindigkeitsfeldern in einer thermischen Konvektionsströmung mit ungekapselten Flüssigkristalltracern. DGLR-Bericht, 4, 1988.

[54] W.J. Hiller and T.A. Kowalewski. Simultaneous measurement of temperature and velocity fields in thermal convective flows. In 4th Int. Symp. on Flow Visualization, Paris, France, 1986.

[55] H. Hu and M.M. Koochesfahani. Molecular tagging velocimetry and thermometry and its application to the wake of a heated circular cylinder. Meas. Sci. Technol., 17:1269-1281, 2006.

[56] P.T. Ireland and T.V. Jones. The response time of a surface thermometer employing encapsulated thermochromic liquid crystals. J. Phys. E: Sci. Instrum., 20:1195-1199, 1987.

[57] P.T. Ireland and T.V. Jones. Liquid crystal measurements of heat transfer and surface shear stress. Meas. Sci. Technol., 11:969-986, 2000.

[58] I. Ishihara, T. Fukui, and R. Matsumoto. Natural convection in a vertical rectangular enclosure with symmetrically localized heating and cooling zones. Int. J. Heat Fluid Flow, 23:366-372, 2002.

[59] H. Johnston and C.R. Doering. Comparison of turbulent thermal convection between conditions of constant temperature and constant flux. Phys. Rev. Lett., 102:064501, 2009.

[60] M. Kaczorowski and C. Wagner. Analysis of the thermal plumes in turbulent Rayleigh-Bénard convection based on well-resolved numerical simulations. J. Fluid Mech., 618:89-112, 2009.

[61] J. Ke and D. Bohl. Effect of experimental parameters and image noise on the error levels in simultaneous velocity and temperature measurements using molecular tagging velocimetry/thermometry (MTV/T). Exp. Fluids, 50:465-478, 2011.

[62] M. Körner, O. Shishkina, C. Wagner, and A. Thess. Properties of large-scale flow structures in an isothermal ventilated room. Build. Environ., 59:563-574, 2013.

[63] T.A. Kowalewski. Particle image velocimetry and thermometry using liquid crystals. In $8 m e$ colloque nationale de visualisation et de traitment d'images en mecanique des fluids, Toulouse, France, 1999. ENSICA.

[64] M. Kühn, J. Bosbach, and C. Wagner. Experimental parametric study of forced and mixed convection in a passenger aircraft mock-up. Build. Environ., 44:961-970, 2009.

[65] M. Kühn, K. Ehrenfried, J. Bosbach, and C. Wagner. Characteristics of large volume tomographic particle image velocimetry using helium filled soap bubbles in forced and thermal convection. In 15th Int. Symp. on Applications of Laser Techniques to Fluid Mechanics, Lisbon, Portugal, 2010.

[66] M. Kühn, K. Ehrenfried, J. Bosbach, and C. Wagner. Large-scale tomographic particle image velocimetry using helium-filled soap bubbles. Exp. Fluids, 50:929-948, 2011.

[67] M. Kühn, K. Ehrenfried, J. Bosbach, and C. Wagner. Large-scale tomographic PIV in forced and mixed convection using a parallel SMART version. Exp. Fluids, 53:91-103, 2012.

[68] S. Kuhn and P. Rudolf von Rohr. Experimental investigation of mixed convective flow over a wavy wall. Int. J. Heat Fluid Flow, 29:94-106, 2008. 
[69] P.F. Linden. The fluid mechanics of natural ventilation. Annu. Rev. Fluid Mech., 31:201-238, 1999.

[70] J.T. Lir and T.F. Lin. Visualization of roll patterns in Rayleigh-Bénard convection of air in a rectangular shallow cavity. Int. J. Heat Mass Transfer, 44:2889-2902, 2001.

[71] D. Lohse and K.-Q. Xia. Small-scale properties of turbulent Rayleigh-Bénard convection. Annu. Rev. Fluid Mech., 42:335-364, 2009.

[72] A. Maystrenko, C. Resagk, and A. Thess. Structure of the thermal boundary layer for turbulent Rayleigh-Bénard convection of air in a long rectangular enclosure. Phys. Rev. E, 75:066303, 2007.

[73] P.K. Mishra, A.K. De, M.K. Verma, and V. Eswaran. Dynamics of reorientations and reversals of large-scale flow in Rayleigh-Bénard convection. J. Fluid Mech., 668:480-499, 2011.

[74] X. Nicolas, J.-M. Luijkx, and J.-K. Platten. Linear stability of mixed convection flows in horizontal rectangular channels of finite transversal extension heated from below. Int. J. Heat Mass Transfer, 43:589-610, 2000.

[75] Nobelprize.org. $\quad$ Liquid crystals. $\quad$ Nobel Media AB 2013. http://www.nobelprize.org/educational/physics/liquid_crystals/ (15 Jan 2014).

[76] Nobelprize.org. Press release: The 1991 nobel prize in physics. Nobel Media AB 2013. http://www.nobelprize.org/nobel_prizes/physics/laureates/1991/ (15 Jan 2014).

[77] A. Oberbeck. Über die Wärmeleitung der Flüssigkeiten bei Berücksichtigung der Strömungen in Folge von Temperaturdifferenzen. Ann. Phys. (Berlin), 243(6):271-292, 1879.

[78] OSRAM. Opto Semiconductors GmbH. http://catalog.osram-os.com (19 Nov 2012).

[79] M. Petroff. http://apod.nasa.gov/apod/ap090824.html (3 Mar 2014).

[80] M. Petroff. http://creativecommons.org/licenses/by-sa/3.0/ (3 Mar 2014).

[81] B. Podvin and A. Sergent. Proper orthogonal decomposition investigation of turbulent Rayleigh-Bénard convection in a rectangular cavity. Phys. Fluids, 24:105106, 2012.

[82] L. Prandtl. Der Luftwiderstand von Kugeln. Nachrichten der Gesellschaft der Wissenschaften zu Göttingen, Mathematisch-physikalische Klasse, pages 177-190, 1914.

[83] B.A. Puthenveettil, G.S. Gunasegarane, Y.K. Agrawal, D. Schmeling, J. Bosbach, and J.H. Arakeri. Length of near-wall plumes in turbulent convection. J. Fluid Mech., 685:335-364, 2011.

[84] M. Raffel, C.E. Willert, S.T. Wereley, and J. Kompenhans. Particle Image Velocimetry - a practical guide. Springer-Verlag, 2nd edition, 2007.

[85] Y. Rao and S. Zang. Calibrations and the measurement uncertainty of wide-band liquid crystal thermography. Meas. Sci. Technol., 21:015105, 2010.

[86] Lord Rayleigh. On the instability of jets. Proc. Lond. Math. Soc., 10:4-13, 1878.

[87] Lord Rayleigh. On convection currents in a horizontal layer of fluid, when the higher temperature is on the under side. Phil. Mag., 32:529-546, 1916.

[88] F. Reinitzer. Beiträge zur Kenntniss des Cholesterins. Monatshefte für Chemie, 9:421-441, 1888. 
[89] C. Resagk, R. du Puits, A. Thess, F.V. Dolzhansky, S. Grossmann, F. Fontenele Araujo, and D. Lohse. Oscillations of the large scale wind in turbulent thermal convection. Phys. Fluids, 18:095105, 2006.

[90] P.H. Roberts and E.M. King. On the genesis of the earth's magnetism. Rep. Prog. Phys., 76:096801, 2013.

[91] T. Roesgen and R. Totaro. A statistical calibration technique for thermochromic liquid crystals. Exp. Fluids, 33:732-734, 2002.

[92] S.M. Saeidi and J.M. Khodadadi. Forced convection in a square cavity with inlet and outlet ports. Int. J. Heat Mass Transfer, 49:1896-1906, 2006.

[93] D. Schiepel, 2013. personal communication.

[94] D. Schmeling, J. Bosbach, and C. Wagner. Development of a particle generator for combined particle image thermo- and velocimetry in air. In 15th Int. Symp. on Applications of Laser Techniques to Fluid Mechanics, Lisbon, Portugal, 2010.

[95] D. Schmeling, J. Bosbach, and C. Wagner. Feasibility of combined PIT and PIV in mixed convective air flows. In 9th Int. Symp. on Particle Image Velocimetry, Kobe, Japan, 2011.

[96] D. Schmeling, J. Bosbach, and C. Wagner. Periodic and quasiperiodic temperature oscillations in mixed convection. In 23rd Int. Congress of Theoretical and Applied Mechanics, Beijing, China, 2012.

[97] D. Schmeling, J. Bosbach, and C. Wagner, 2013. See supplemental material at http://link. springer. com/article/10.1007/s00348-013-1517-3.

[98] D. Schmeling, J. Bosbach, and C. Wagner. Oscillations of the large-scale circulation in turbulent mixed convection in a closed rectangular cavity. Exp. Fluids, 54:1517, 2013.

[99] D. Schmeling, J. Bosbach, and C. Wagner, 2014. See supplemental material at http://stacks.iop.org/MST/25/035302/mmedia.

[100] D. Schmeling, J. Bosbach, and C. Wagner. Measurements of the dynamics of thermal plumes in turbulent mixed convection based on combined PIT and PIV. In preparation for Exp. Fluids, 2014.

[101] D. Schmeling, J. Bosbach, and C. Wagner. Simultaneous measurement of temperature and velocity fields in convective air flows. Meas. Sci. Technol., 25:035302, 2014.

[102] D. Schmeling, J. Bosbach, and C. Wagner. Temperature oscillations in turbulent mixed convective air flows. In M. O. Deville, J.-L. Estivalezes, V. Gleize, T.-H. Lê, M. Terracol, and S. Vincent, editors, Turbulence and Interactions, volume 125 of Notes on Numerical Fluid Mechanics and Multidisciplinary Design, pages 157-163. Springer-Verlag, 2014.

[103] D. Schmeling, M. Czapp, J. Bosbach, and C. Wagner. Development of Combined Particle Image Velocimetry and Particle Image Thermography for Air Flows, volume 4 of 14th Int. Heat Transfer Conference, pages 57-64. ASME, Washington, DC, USA, 2010.

[104] D. Schmeling, A. Westhoff, M. Kühn, J. Bosbach, and C. Wagner. Flow structure formation of turbulent mixed convection in a closed rectangular cavity. In A. Dillmann, G. Heller, M. Klaas, H.-P. Kreplin, W. Nitsche, and W. Schröder, editors, New Results in Numerical and Experimental Fluid Mechanics VII, volume 112 of Notes on Numerical Fluid Mechanics and Multidisciplinary Design, pages 571-578. Springer-Verlag, 2010. 
[105] D. Schmeling, A. Westhoff, M. Kühn, J. Bosbach, and C. Wagner. Large-scale flow structures and heat transport of turbulent forced and mixed convection in a closed rectangular cavity. Int. J. Heat Fluid Flow, 32:889-900, 2011.

[106] A. Sergent and P. Le Quéré. Long time evolution of large-scale patterns in a rectangular Rayleigh-Bénard cell. J. Phys.: Conf. Ser., 318:082010, 2011.

[107] O. Shishkina and C. Wagner. Measured local heat transport in turbulent Rayleigh-Bénard convection. Phys. Rev. Lett., 90(7):074501, 2003.

[108] O. Shishkina and C. Wagner. Analysis of sheet-like thermal plumes in turbulent RayleighBénard convection. J. Fluid Mech., 599:383-404, 2008.

[109] O. Shishkina and C. Wagner. A numerical study of turbulent mixed convection in an enclosure with heated rectangular elements. J. Turb., 13:1-21, 2012.

[110] J.J.M. Sillekens, C.C.M. Rindt, and A.A. Van Steenhoven. Developing mixed convection in a coiled heat exchanger. Int. J. Heat Mass Transfer, 41:61-72, 1998.

[111] J.J.M. Sillekens, C.C.M. Rindt, and A.A. Van Steenhoven. Development of laminar mixed convection in a horizontal square channel with heated side walls. Int. J. Heat Fluid Flow, 19:270-281, 1998.

[112] L. Sirovich. Turbulence and the dynamics of coherent structures, part i: coherent structures. Quart. Appl. Math., 45:561-571, 1987.

[113] L. Sirovich. Turbulence and the dynamics of coherent structures, part ii: symmetries and transformation. Quart. Appl. Math., 45:573-582, 1987.

[114] C.R. Smith, D.R. Sabatino, and T.J. Praisner. Temperature sensing with thermochromic liquid crystals. Exp. Fluids, 30:190-201, 2001.

[115] S. Someya, Y. Li, K. Ishii, and K. Okamoto. Combined two-dimensional velocity and temperature measurements of natural convection using a high-speed camera and temperaturesensitive particles. Exp. Fluids, 50:65-73, 2011.

[116] E.A. Spiegel. Convection in stars, i. basic boussinesq convection. Annu. Rev. Astro. Astrophys., 9:323-352, 1971.

[117] J.H. Spurk. Dimensionsanalyse in der Strömungslehre. Springer-Verlag, 1992.

[118] K.R. Sreenivasan, A. Bershadskii, and J.J. Niemela. Mean wind and its reversal in thermal convection. Phys. Rev. E, 65:056306, 2002.

[119] C. Stamatopoulos, D.S. Mathioulakis, and A. Katsamouris. Mixed convection flow investigation in a rectangular horizontal tube stenosis via liquid crystal thermography and planar PIV. Exp. Therm. Fluid Sci., 35:375-386, 2011.

[120] A.N.P. Stevens. Factors affecting global climate. Nature Education Knowledge, 3(10):18, 2012 .

[121] B. Stevens. Atmospheric moist convection. Annu. Rev. Earth Planet. Sci., 33:605-643, 2005.

[122] R.J.A.M. Stevens, E.P. van der Poel, S. Grossmann, and D. Lohse. The unifying theory of scaling in thermal convection: the updated prefactors. J. Fluid Mech., 730:295-308, 2013.

[123] K. Sugiyama, E. Calzavarini, S. Grossmann, and D. Lohse. Non-oberbeck-boussinesq effects in two-dimensional Rayleigh-Bénard convection in glycerol. Europhys. Lett., 80:34002, 2007. 
[124] K. Sugiyama, R. Ni, R.J.A.M. Stevens, T.S. Chan, S.-Q. Zhou, H.-D. Xi, C. Sun, S. Grossmann, K.-Q. Xia, and D. Lohse. Flow reversals in thermally driven turbulence. Phys. Rev. Lett., 105:034503, 2010.

[125] C. Sun, K.-Q. Xia, and P. Tong. Three-dimensional flow structures and dynamics of turbulent thermal convection in a cylindrical cell. Phys. Rev. E, 72:026302, 2005.

[126] W.J. Hiller T.A. Kowalewski and G. de Vahl Davis. Computational and experimental visualisation in heat and mass transfer problems. In 1st Japanese-Polish Joint Seminar in Advanced Computer Simulation, pages 60-69, Tokyo, Japan, 1994. University of Tokyo.

[127] P.J. Tackley. Geodynamics: Layer cake or plum pudding? Nat. Geosci., 1:157-158, 2008.

[128] E.J. Tarbuck, F.K. Lutgens, and D. Tasa. Earth science. Prentice Hall, 2000.

[129] G.R. Thompson and J. Turk. Earth Science and the Environment. Saunders College Pub., 2nd edition, 1999.

[130] D.J. Tritton. Physical Fluid Dynamics. Oxford University Press, 2nd edition, 2007.

[131] C. Tropea, J. Foss, and A. Yarin. Handbook of Experimental Fluid Mechanics. Springer-Verlag, 2007.

[132] E.P. van der Poel, R.J.A.M. Stevens, and D. Lohse. Connecting flow structures and heat flux in turbulent Rayleigh-Bénard convection. Phys. Rev. E, 84:045303, 2011.

[133] E.P. van der Poel, R.J.A.M. Stevens, K. Sugiyama, and D. Lohse. Flow states in twodimensional Rayleigh-Bénard convection as a function of aspect-ratio and Rayleigh number. Phys. Fluids, 24:085104, 2012.

[134] Verein Deutscher Ingenieure, VDI - Gesellschaft Verfahrenstechnik und Chemieingenieurwesen (GVC), editor. VDI - Wärmeatlas. Springer-Verlag, 10th edition, 2006.

[135] L. Da Vinci. Royal Collection Trust / (CHer Majesty Queen Elizabeth II. http://www.royalcollection.org.uk, RCIN 912660, (4 Mar 2014).

[136] S. Wagner and O. Shishkina. Aspect-ratio dependency of Rayleigh-Bénard convection in box-shaped containers. Phys. Fluids, 25:085110, 2013.

[137] S. Wagner, O. Shishkina, and C. Wagner. Boundary layers and wind in cylindrical RayleighBénard cells. J. Fluid Mech., 697:336-366, 2012.

[138] S. Weiss and G. Ahlers. Turbulent Rayleigh-Bénard convection in a cylindrical container with aspect ratio $\Gamma=0.50$ and Prandtl number $\operatorname{Pr}=4.38$. J. Fluid Mech., 676:5-40, 2011.

[139] A. Westhoff. Spatial scaling of large-scale circulations and heat transport in turbulent mixed convection. Phd thesis, Georg-August-University, Göttingen, Germany, 2012.

[140] A. Westhoff, J. Bosbach, D. Schmeling, and C. Wagner. Experimental study of low-frequency oscillations and large-scale circulations in turbulent mixed convection. Int. J. Heat Fluid Flow, 31:794-804, 2010.

[141] A. Westhoff, N. Grabinski, J. Bosbach, and C. Wagner. Scaling of turbulent mixed convection under high pressure. In 7th Int. Symp. on Turbulence and Shear Flow Phenomena, Garching, Germany, 2007. 
[142] A. Westhoff, D. Schmeling, J. Bosbach, and C. Wagner. Oscillations of large-scale structures in turbulent mixed convection in a rectangular enclosure, pages 533-536. Advance in Turbulence XII - Proceedings of the 12th Euromech European Turbulence Conference. Springer-Verlag, 2009 .

[143] R. Wiberg and N. Lior. Errors in thermochromic liquid crystal thermometry. Rev. Sci. Instrum., 75(9):2985-2994, 2004.

[144] C. Wieselsberger. Der Luftwiderstand von Kugeln. Zeitschrift für Flugtechnik und Motorluftschiffahrt, 5:140-145, 1914.

[145] C. Willert, B. Stasicki, J. Klinner, and S. Moessner. Pulsed operation of high-power light emitting diods for imaging flow velocimetry. Meas. Sci. Technol., 21:1-11, 2010.

[146] P. Tong X.-D. Shang, X.-L. Qiu and K.-Q. Xia. Local heat fluxes in turbulent Rayleigh-Bénard convection. Phys. Fluids, 19:085107, 2007.

[147] H.-D. Xi, S. Lam, and K.-Q. Xia. From laminar plumes to organized flows: the onset of large-scale circulation in turbulent thermal convection. J. Fluid Mech., 503:47-56, 2004.

[148] H.-D. Xi and K.-Q. Xia. Azimuthal motion, reorientation, cessation, and reversal of the large-scale circulation in turbulent thermal convection: A comparative study in aspect ratio one and one-half geometries. Phys. Rev. E, 78:036326, 2008.

[149] H.-D. Xi and K.-Q. Xia. Flow mode transitions in turbulent thermal convection. Phys. Fluids, 20:055104, 2008.

[150] H.-D. Xi, S.-Q. Zhou, Q. Zhou, T.-S. Chan, and K.-Q. Xia. Origin of the temperature oscillation in turbulent thermal convection. Phys. Rev. Lett., 102:044503, 2009.

[151] K.-Q. Xia, C. Sun, and S.-Q. Zhou. Particle image velocimetry measurement of the velocity field in turbulent thermal convection. Phys. Rev. E, 68:066303, 2003.

[152] Q. Zhou, C. Sun, and K.-Q. Xia. Morphological evolution of thermal plumes in turbulent Rayleigh-Bénard convection. Phys. Rev. Lett., 98:074501, 2007.

[153] Q. Zhou and K.-Q. Xia. Physical and geometrical properties of thermal plumes in turbulent Rayleigh-Bénard convection. New J. Phys., 12:075006, 2010.

[154] G. Zocchi, E. Moses, and A. Libchaber. Coherent structures in turbulent convection, an experimental study. Physica A, 166:387-407, 1990. 



\section{APPENDIX A}

\section{Curriculum Vitae}

\section{Dipl. Phys. Daniel Schmeling}

geb. 13.04.1983 in Oldenburg (Oldb)

Nationalität: deutsch

Familienstand: $\quad$ verheiratet, 1 Kind

Adresse: Herzberger Landstr. 30

37085 Göttingen

Telefon: $\quad 01607563379$

E-Mail: danielschmeling@gmx.de

\section{Wissenschaftlicher Werdegang}

seit 2012

2009-2012

seit 2009

November 2008

2003-2008

Juni 2002

Konferenzen
Anstellung als Mitarbeiter im wissenschaftlichen Dienst am Deutschen Zentrum für Luft- und Raumfahrt e.V. in Göttingen

Anstellung als Doktorand am Deutschen Zentrum für Luft- und Raumfahrt e.V. in Göttingen

Promotionsstudiengang (GAUSS), Georg-August-Universität Göttingen Diplom in Physik, Diplomarbeit: "Experimentelle Untersuchung von Strukturbildung und Wärmetransport in turbulenter Mischkonvektion". Georg-August-Universität Göttingen, Note: sehr gut Studium der Physik, Georg-August-Universität Göttingen

Abitur, Gymnasium am Treckfahrtstief Emden, Note: sehr gut 
- $23^{\text {rd }}$ Int. Congress of Theoretical and Applied Mechanics, Peking, China, 2012. (Vortrag)

- $3^{\text {rd }}$ Int. Conference on Turbulence and Interactions, La Saline-lesBains, La Réunion, 2012. (Vortrag)

- $5^{\text {th }}$ European Postgraduate Fluid Dynamics Conference, Göttingen, Deutschland, 2011. (Poster)

- $9^{\text {th }}$ Int. Symposium on Particle Image Velocimetry, Kobe, Japan, 2011. (Vortrag und Poster)

- $14^{\text {th }}$ Int. Heat Transfer Conference, Washington D.C., USA, 2010. (Poster)

- $15^{\text {th }}$ Int. Symposium on Applications of Laser Techniques to Fluid Mechanics, Lissabon, Portugal, 2010. (Vortrag)

\section{Referierte Veröffentlichungen}

internationale Zeitschriften

- D. Schmeling, J. Bosbach, C. Wagner: „Simultaneous measurement of temperature and velocity fields in convective air flows". Meas. Sci. Technol., 25, 2014, 035302.

- D. Schmeling, J. Bosbach, C. Wagner: „Oscillations of the largescale circulation in turbulent mixed convection in a closed rectangular cavity“. Exp. Fluids, 54, 2013, 1517.

- B.A. Puthenveettil, G.S. Gunasegarane, Y.K. Agrawal, D. Schmeling, J. Bosbach, J.H. Arakeri: „Length of near-wall plumes in turbulent convection“. J. Fluid Mech., 685, 2011, pp. 335-364.

- D. Schmeling, A. Westhoff, M. Kühn, J. Bosbach, C.Wagner: „Large-scale flow structures and heat transport of turbulent forced and mixed convection in a closed rectangular cavity". Int. Journal of Heat and Fluid Flow, 32, 2011, pp. 889-900.

- A. Westhoff, J. Bosbach, D. Schmeling, C. Wagner: „Experimental study of low-frequency oscillations and large-scale circulations in turbulent mixed convection". Int. Journal of Heat and Fluid Flow, 31, 2010, pp. 794-804. 
Buchbeiträge

- D. Schmeling, J. Bosbach, C. Wagner: „Temperature oscillations in turbulent mixed convective air flows". In: „Turbulence and Interactions“, volume 125 of „Notes on Numerical Fluid Mechanics and Multidisciplinary Design", Springer-Verlag Berlin Heidelberg, 2014, pp. 157-163.

- D. Schmeling, M. Czapp, J. Bosbach, C. Wagner: „Development of combined particle image velocimetry and particle image thermography for air flows". In: Proceedings of $14^{\text {th }}$ Int. Heat Transfer Conference, Volume 4, Washington D.C., USA, ASME, 2010, pp. 57-64..

- D. Schmeling, A. Westhoff, M. Kühn, J. Bosbach, C. Wagner: „Flow structure formation of turbulent mixed convection in a closed rectangular cavity“. In: „New Results in Numerical and Experimental Fluid Mechanics VII“, volume 112 of „Notes on Numerical Fluid Mechanics and Multidisciplinary Design“, Springer-Verlag Berlin Heidelberg, 2010, pp. 571-578.

- A. Westhoff, D. Schmeling, J. Bosbach, C. Wagner: „Oscillations of large-scale structures in turbulent mixed convection in a rectangular enclosure“. In: „Advance in Turbulence XII - Proceedings of the $12^{\text {th }}$ Euromech European Turbulence Conference“, Springer-Verlag Berlin Heidelberg, 2009, pp. 533-536.

\section{Lehrtätigkeiten}

Betreuung von Experimenten des „Grundpraktikums Physik“ der Georg-August-Universität Göttingen

2010 - 2011 Betreuung von Experimenten des „Physikalischen Praktikums für Studenten mit Physik als Nebenfach" der Georg-August-Universität Göttingen

2009 - 2010 Betreuung von Experimenten des ,Strömungsmechanischen Praktikums" der Georg-August-Universität Göttingen

2007 - 2008 Betreuung von Experimenten im School_Lab des Deutschen Zentrums für Luft und Raumfahrt e.V. in Göttingen

\section{Tätigkeiten am DLR}

Mitarbeit im „Enhanced Cabin Comfort Computations“ (ECCO) Projekt des Deutschen Zentrums für Luft- und Raumfahrt e.V. Mitarbeit im „Comfortable and Silent Cabin Plus“ (CoSiCab+) Projekt des Deutschen Zentrums für Luft- und Raumfahrt e.V. 


\section{Gutachtertätigkeiten}

Journal of Fluid Mechanics

ASME/JSME 2011 8th Thermal Engineering Joint Conference, AJTEC 2011

\section{Auslandsaufenthalte}

$1999-2000 \quad$ KMS Highschool in Kerkhoven, USA

\section{Preise / Auszeichnungen}

„Kobe Award“: Bestes Poster auf der Konferenz: „Combined PIT and PIV in mixed convective air flows". Poster competition for young researchers, $9^{\text {th }}$ Int. Symposium on Particle Image Velocimetry, Kobe, Japan, 20. Juli 2011.

(Daniel Schmeling)

Göttingen, 24. Juli 2014 